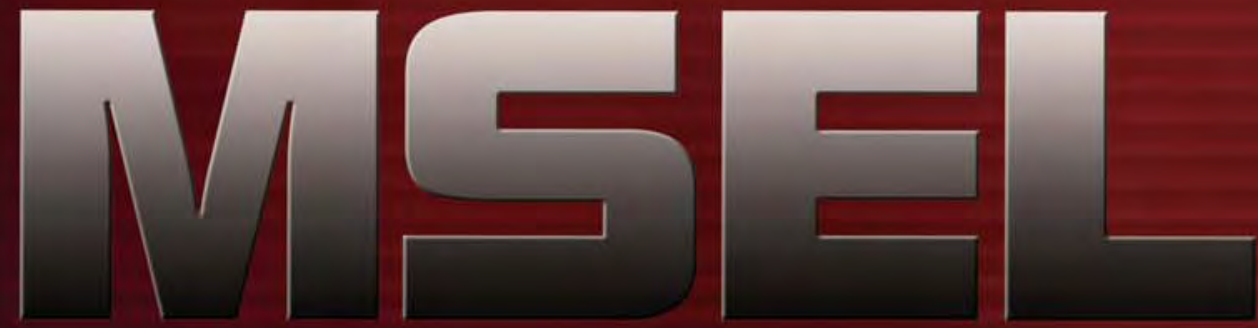

Materials Science and Engineering Laboratory

\title{
FY 2005 Programs and Accomplishments
}
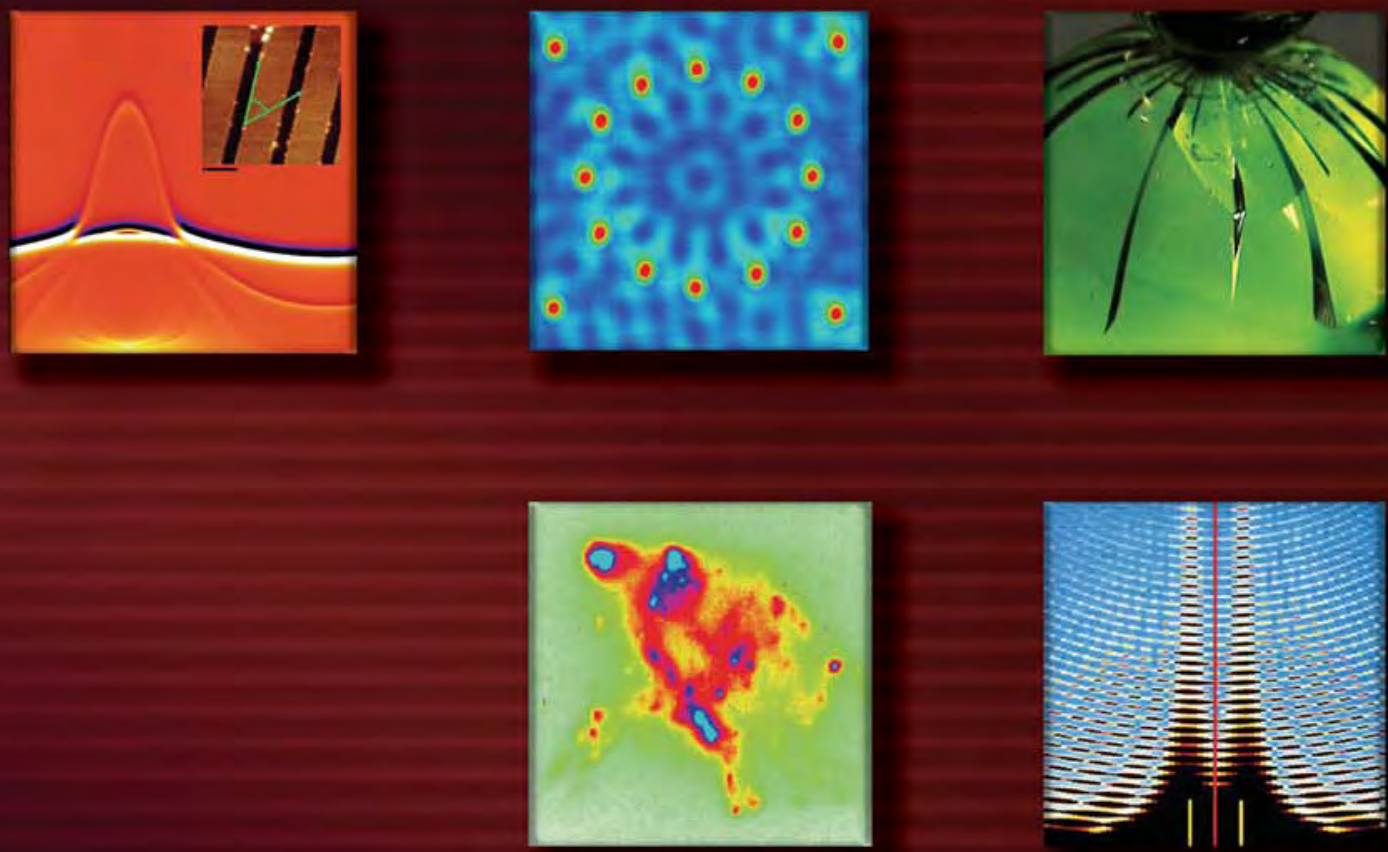

\section{NGT}

\section{National Institute of}

Standards and Technology

Technology Administration

U.S. Department of Commerce

NISTIR 7248

December 2005 

National Institute of

Standards and Technology

William Jeffrey

Director

Technology

Administration

Michelle O'Neill

Acting Under Secretary of

Commerce for Technology

U.S. Department

of Commerce

Carlos M. Gutierrez

Secretary

\section{Materials Science and \\ Engineering Laboratory}

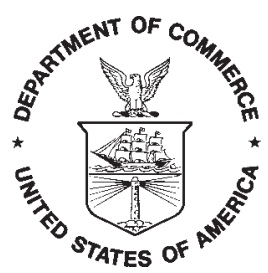

\section{FY 2005 Programs and ACCOMplishmENTS}

\section{Ceramics, Materials Reliability, Metallurgy, NIST Center for Neutron Research, and Polymers Divisions}

Richard F. Kayser, Director

Stephen W. Freiman, Deputy Director

NISTIR 7248

December 2005 
Certain commercial entities, equipment, or materials may be identified in this document in order to describe an experimental procedure or concept adequately. Such identification is not intended to imply recommendation or endorsement by the National Institute of Standards and Technology, nor is it intended to imply that the entities, materials, or equipment are necessarily the best available for the purpose. 


\section{Table of Contents}

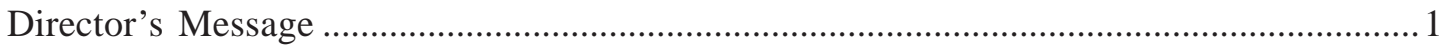

Materials Science and Engineering Laboratory Organization Chart ........................................ 2

Year in Review

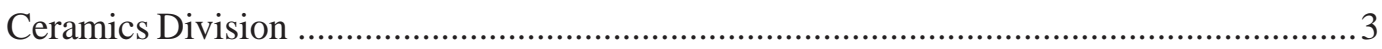

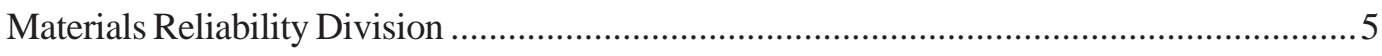

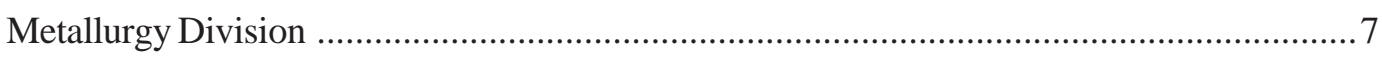

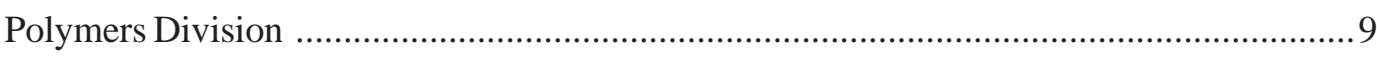

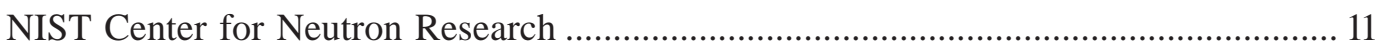

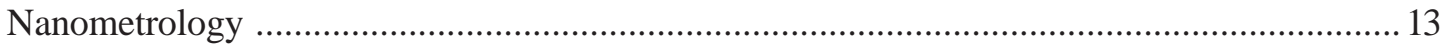

Mechanical Metrology for Small-Scale Structures …...................................................... 14

Nanomechanics: Atomistics in Modeling and Experiments ............................................ 15

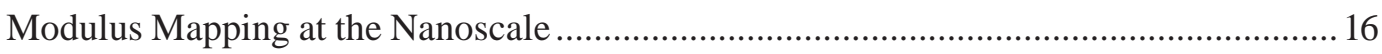

Reference Specimens for SPM Nanometrology …....................................................... 17

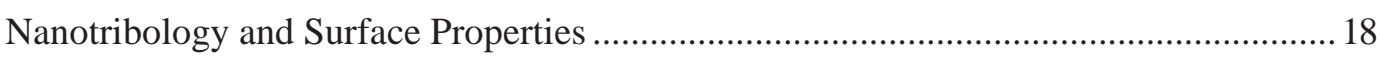

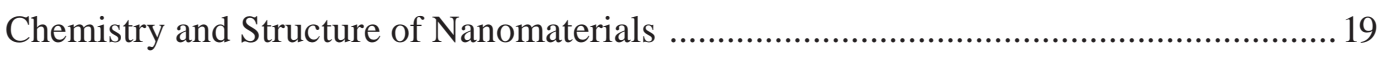

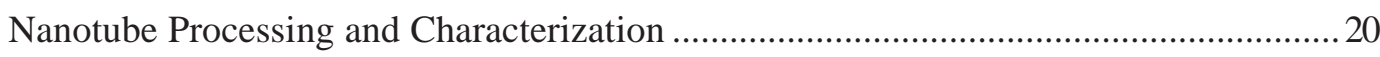

Carbon Nanotube Applications: The Role of Nanotube Alignment .................................. 21

Combinatorial Adhesion and Mechanical Properties ......................................................2 22

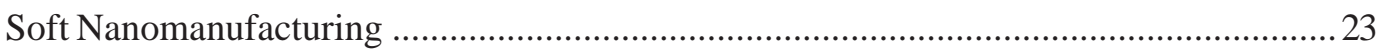

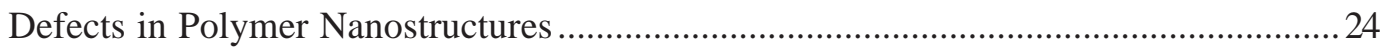

Critical Dimension Small Angle X-Ray Scattering …..................................................... 25

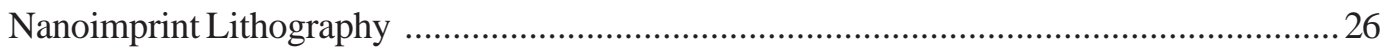

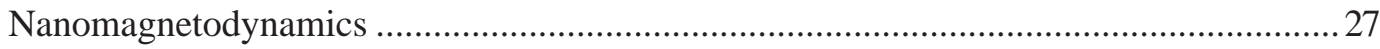

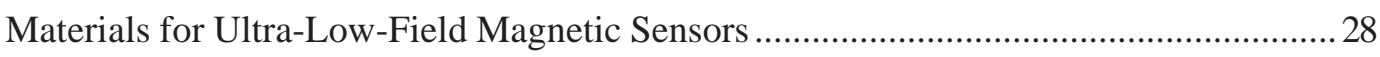

A New Type of Antisymmetric Magnetoresistance

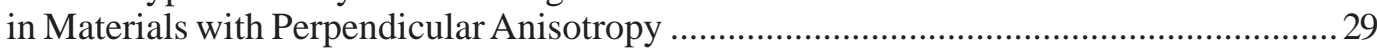

Nanostructure Fabrication Processes:

Surface \& Growth Stress During Thin Film Electrodeposition ........................................30

Multiscale Modeling of Quantum Dots in Semiconductors ................................................ 31

Brillouin Light Scattering:

Dynamic Elastic and Magnetic Properties of Nanostructures .......................................... 32 


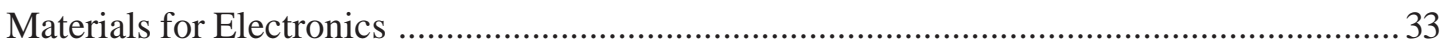

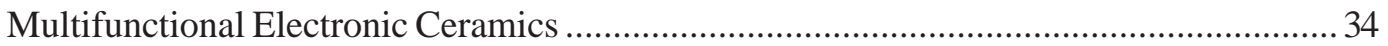

Spectroscopy, Diffraction, and Imaging of Electronic Materials ....................................... 35

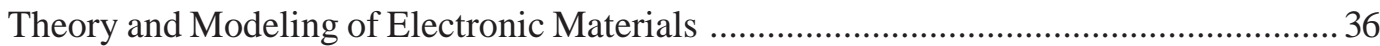

Advanced Materials for Energy Applications ...................................................................... 37

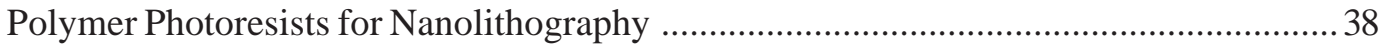

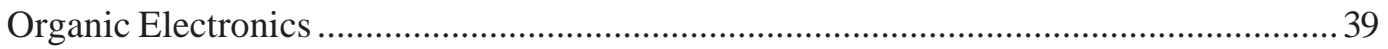

Nanoporous Low-k Dielectric Constant Thin Films ........................................................ 40

Electrical Methods for Mechanical Testing ................................................................... 41

Thermochemical Metrology of Interfacial Stabilities ......................................................... 42

Combinatorial Metal Selection for Catalytic Growth

of $\mathrm{ZnO}$ Semiconductor Nanowires ................................................................................... 43

Advanced Processes and Materials for On-Chip Interconnects .......................................44

Pb-free Surface Finishes: Sn Whisker Growth .................................................................. 45

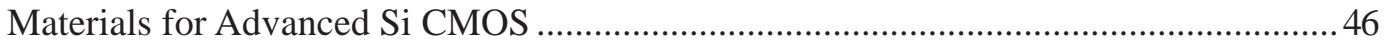

Metrology and Standards for Electronic and

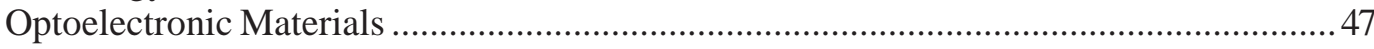

Nano-Structured Materials for Sensors and

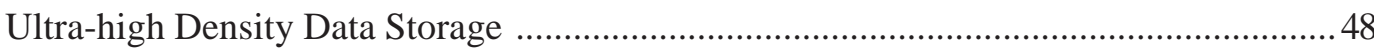

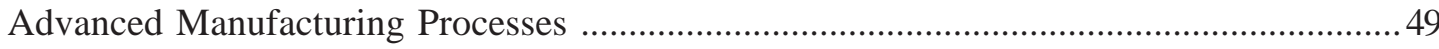

Mechanisms for Delivery of Thermodynamic and Kinetic Data .....................................5 51

FiPy: An Adaptable Framework for Phase Field

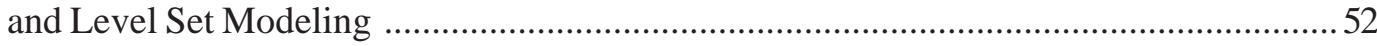

Metrology Tools to Accelerate Industrial Development

of Solid State Hydrogen Storage Materials .......................................................................... 53

Fundamental Nature of Crack Tips in Glass .................................................................... 54

Hardness Standardization: Rockwell, Vickers, Knoop ...................................................... 55

NIST Combinatorial Methods Center

Pioneer and Partner in Accelerated Materials Research .......................................... 56

Polymer Formulations:

Materials Processing and Characterization on a Chip ..................................................... 57

Quantitative Polymer Mass Spectrometry ....................................................................... 58

Standard Tests and Data for Sheet Metal Formability .................................................... 59

Microstructural Origins of Surface Roughening

and Strain Localizations

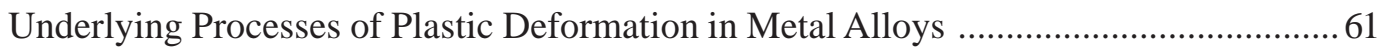

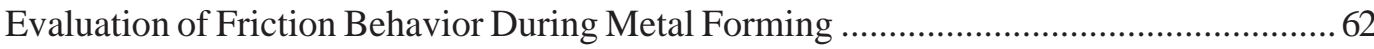


Biomaterials 63

Combinatorial Methods for Rapid Characterization of Cell-Surface Interactions 64

Cellular Level Measurements 65

Cell Response to Tissue Scaffold Morphology 66

3-Dimensional In Situ Imaging for Tissue Engineering:

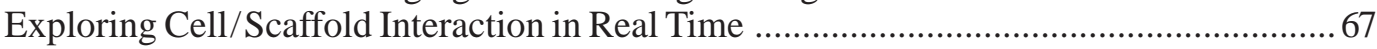

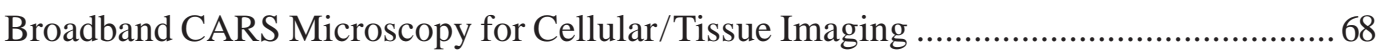

Response of Tissues and Tissue-Engineered Constructs to Mechanical Stimulation 69

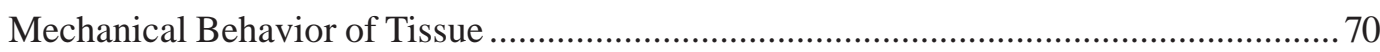

Materials Design for Biomechanical Structures .......................................................... 71

Molecular Design and Combinatorial Characterization of Polymeric Dental Materials

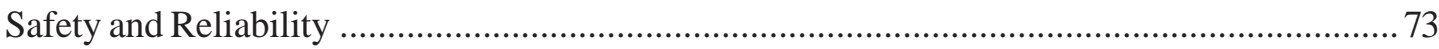

Analysis of Structural Steel from the World Trade Center ............................................... 74

Infrastructure Reliability: Charpy Impact Machine Verification ....................................... 75

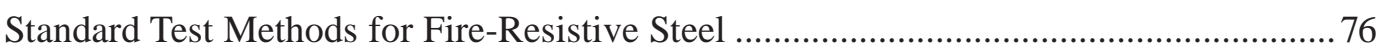

Frangible Bullets and Soft Body Armor ........................................................................ 77

Pipeline Safety: Corrosion, Fracture, and Fatigue ......................................................... 78

Polymer Reliability and Threat Mitigation ........................................................................ 79

Facilities and Capabilities

Databases 91

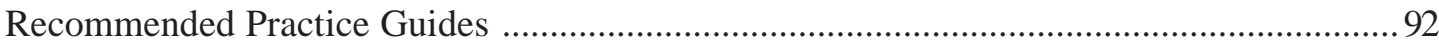




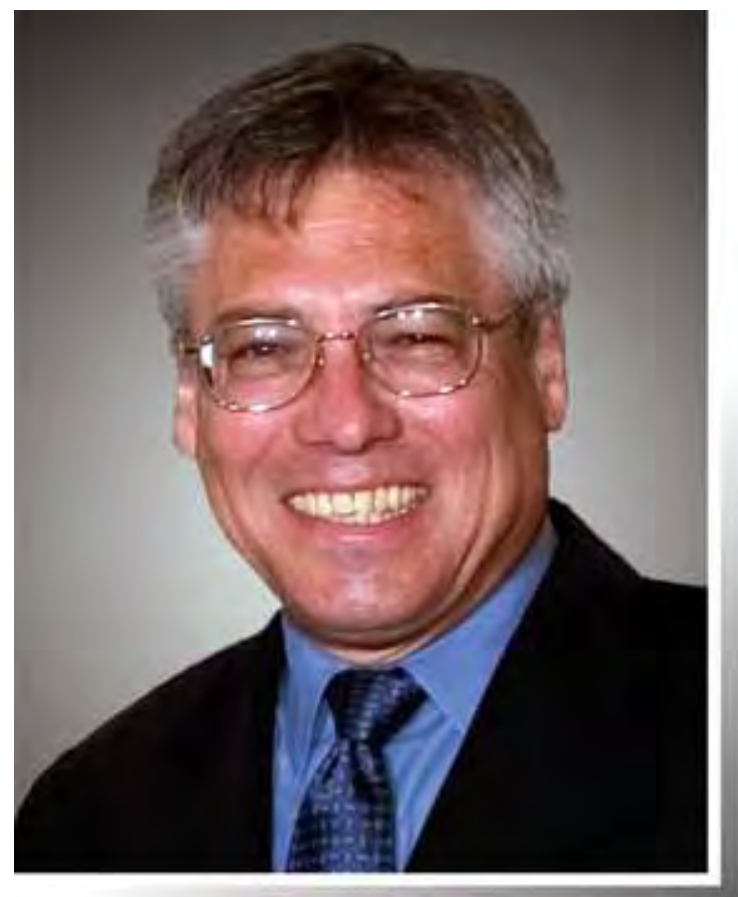

\section{Director's Message}

MSEL works with industry, standards bodies, universities, and other government laboratories to improve the nation's measurements and standards infrastructure for materials.

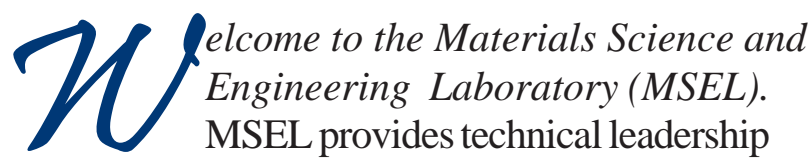
for the nation's materials measurement and standards infrastructure, using expertise in ceramics, polymers, metallurgy, neutron characterization, and materials reliability to anticipate and respond to industry and national needs in areas such as microelectronics, automotive, and health care. The Laboratory also houses the Nation's only fully equipped cold neutron research facility, the NIST Center for Neutron Research.

This document provides a summary of accomplishments that we hope will communicate our commitment to the needs of our customers. Our projects fall into five broad technology focus areas: Nanometrology; Materials for Electronics; Advanced Manufacturing Processes; Biomaterials; and Safety and Reliability. The sections on each of these areas begins with a brief program summary.

\section{Richard F. Kayser}

Director

Materials Science and Engineering Laboratory 


\section{National Institute of Standards and Technology}

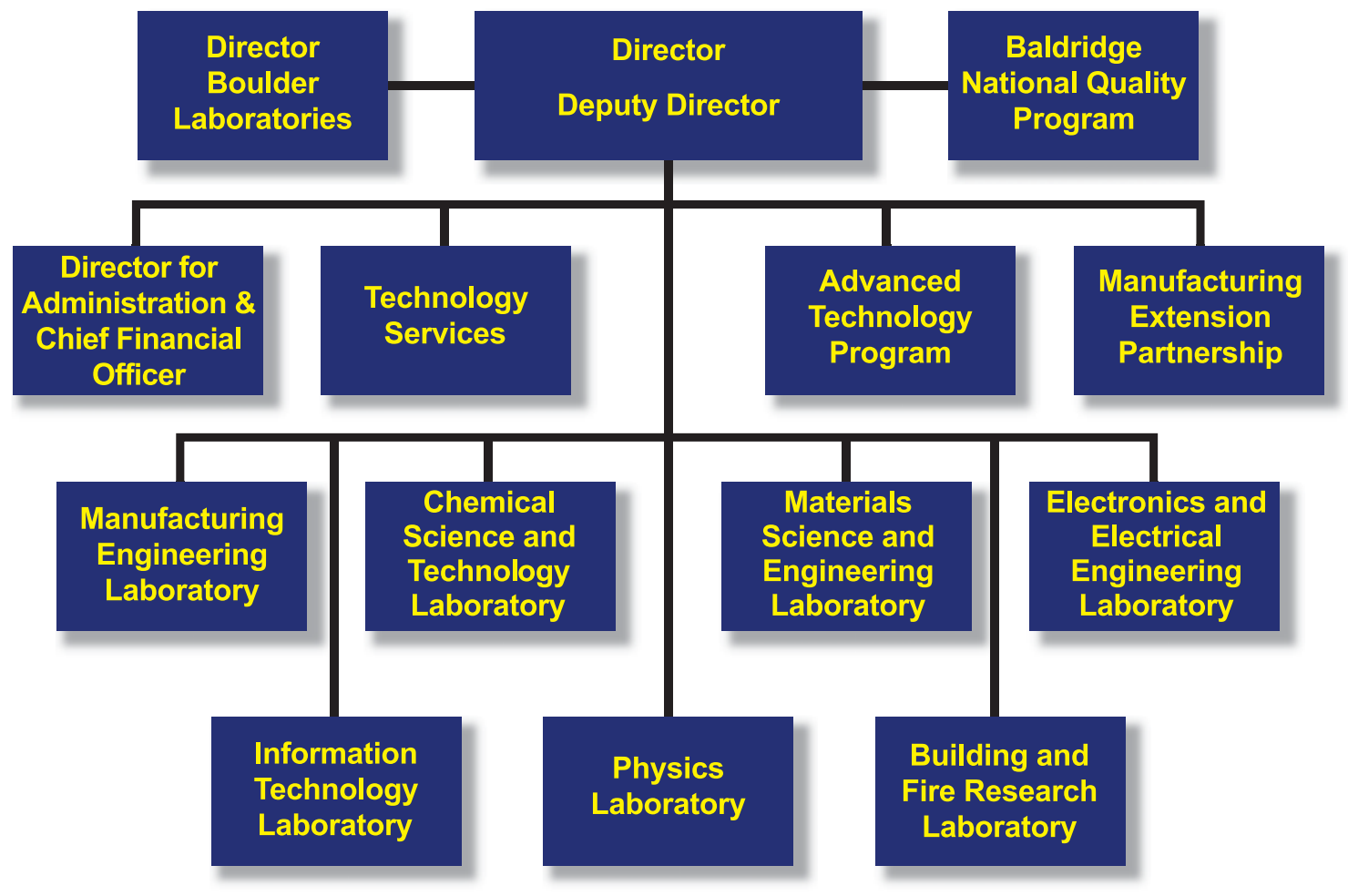

\section{Materials Science and Engineering Laboratory}

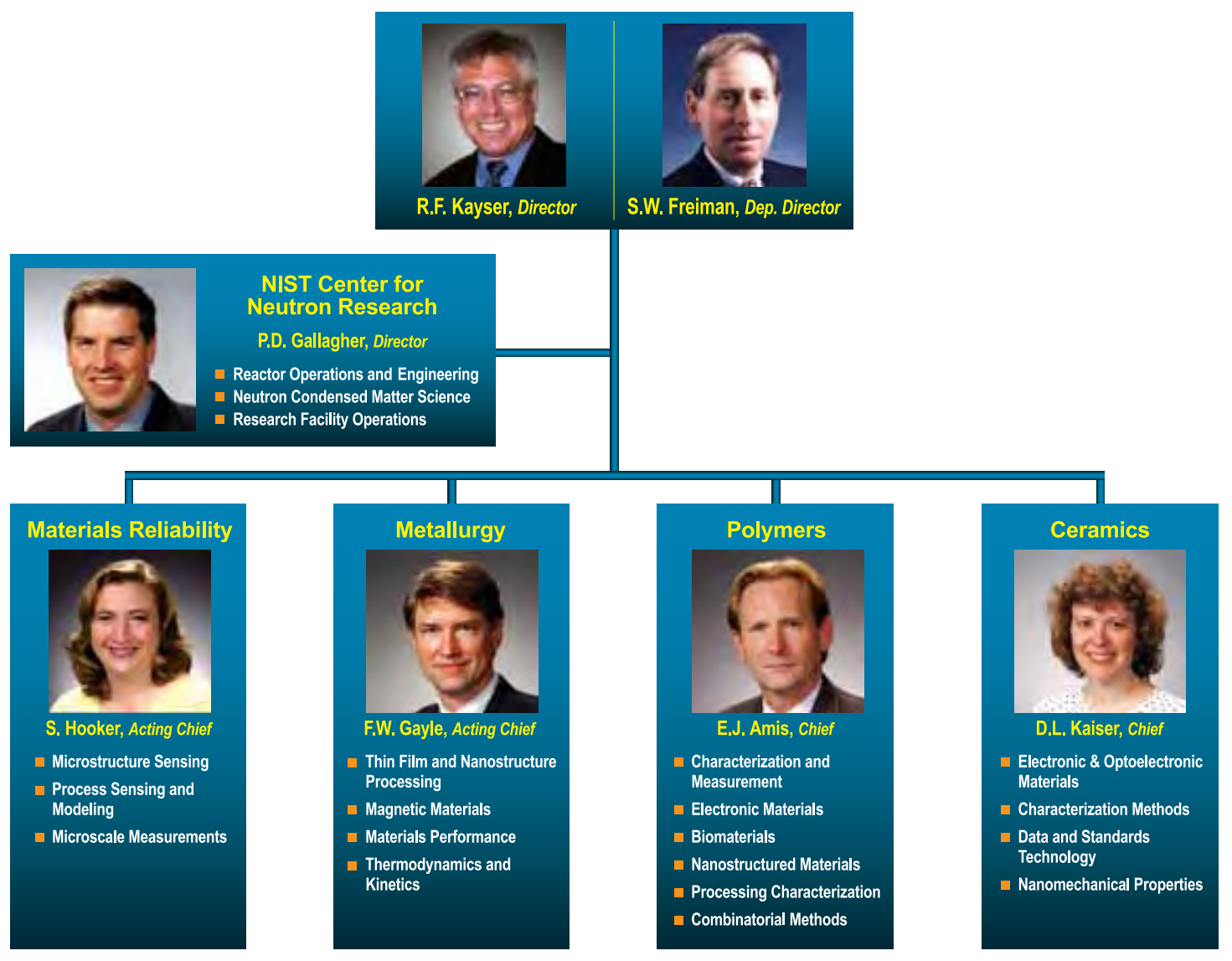




\section{Ceramics Division}

$\mathrm{I}_{\mathrm{n}}^{\mathrm{t}}$ t has been an exciting year in the Ceramics Division for forging new partnerships with industry and other national laboratories, initiating research efforts relevant to emerging nanomaterial systems, and strengthening our ongoing core metrology, data, and standards activities closely aligned with the mission of NIST. Our accomplishments predominately relate to Nanometrology and Materials for Electronics, two of the five program areas in the Materials Science and Engineering Laboratory (MSEL). To crown these achievements, our excellent technical staff members have been honored with a host of prestigious NIST and external awards.

A concerted effort to partner directly with Sematech has led to research activities in advanced metrology to address pressing needs in next-generation semiconductor products. One project is aimed at developing metrology for accurate thin film characterization using x-ray reflectometry, while the other is focused on applying novel combinatorial and synchrotron methods to optimize the interfaces in advanced high-k dielectric CMOS gate stacks critical to the semiconductor industry's 21st century technology roadmap.

A partnership with the National Cancer Institute (NCI) was initiated this year. NCI has awarded NIST a three-year research grant to collaborate with NCI's new Nanotechnology Characterization Laboratory in the development and application of nanoparticle-based systems for cancer prevention, detection, and therapeutics. The Ceramics Division is contributing its long-standing expertise in nanoparticle metrology to this effort by developing measurement methods and protocols for characterizing the size, size distribution, and dispersion of inorganic and organic nanoparticles in aqueous solutions compatible with body fluids.

The Ceramics Division has maintained its strong commitment to two outstanding long-term partnerships aimed at providing the reliable, high-quality data that is fundamentally essential for advanced technology research and development: the celebrated NIST-American Ceramic Society collaboration on phase equilibria diagrams and the collaboration with FIZ Karlsruhe (Germany) on the renowned FIZ-NIST Inorganic Crystal Structure Database.

Ongoing partnerships at DOE synchrotron user facilities have continued to provide high-quality, unique capabilities for structural and chemical characterization of advanced materials. In a joint effort with Sandia National Laboratory, considerable progress has been made to establish a synchrotron-based variable kinetic energy XPS facility at the National Synchrotron Light Source (NSLS), Brookhaven National Laboratory (BNL), the first of its kind in the U.S. For the fifth consecutive year, the soft $x$-ray beamline jointly developed by NIST and Dow Chemical Co. was the most productive soft x-ray facility at NSLS, yielding over 25 publications. Our long-term partnership in UNICAT, a collaborative access team at the Advanced Photon Source (APS), Argonne National Laboratory, has continued to support and improve numerous scattering and diffraction techniques. This partnership will undergo a transition next year as operation and management of the beamline will be assumed by the APS.

The Ceramics Division has continued to support the upgrade and expansion of its unique measurement capabilities. Instruments in the high-resolution x-ray metrology and nanotribology facilities in the NIST Advanced Measurements Laboratory (AML) became fully operational this year and have already yielded results with unprecedented resolution. With the recent modernization of two beamlines at NSLS dedicated to extended x-ray absorption fine structure (EXAFS) and x-ray photoelectron spectroscopy (XPS), the Ceramics Division and its partners have established the capability to perform $\mathrm{x}$-ray absorption spectroscopy spanning all elements in the periodic table. A three-year SBIR project has led to the development of a state-of-the-art multi-element detector at the NSLS soft x-ray beamline, providing an order of magnitude increase in data collection rates.

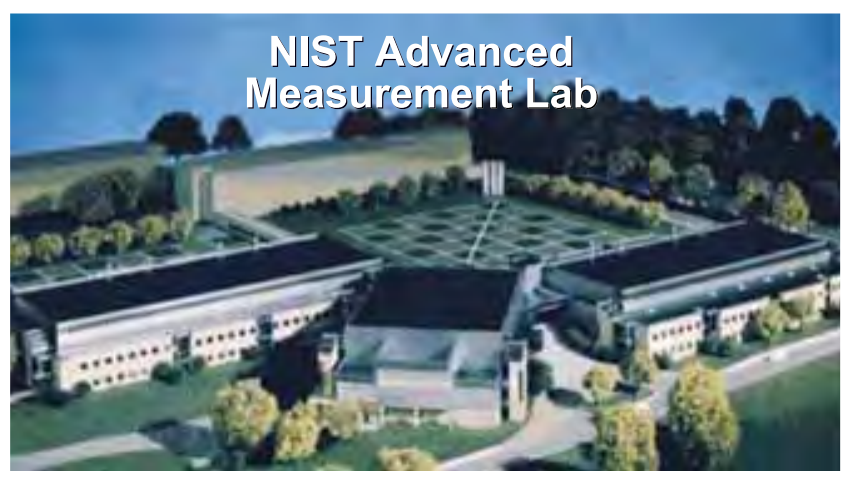

We represent MSEL in the AML, the world's premiere metrology laboratory.

There have been numerous notable scientific achievements across the Division this year; following are several representative research highlights. Ten international leaders worked together to create the IUPAC-NIST Crystal Phase Identifier standard to uniquely identify any chemical compound appearing in an electronic database. This landmark standard is a major step forward towards the worldwide interoperability of crystal structure databases. In collaboration with BNL scientists, our unique near-edge $x$-ray absorption fine structure metrology facility at the NSLS has been employed to characterize the surface order and structure of carbon and boron nitride nanotubes, 
resulting in four refereed journal publications. A feature article reviewing the state-of-the-art in characterizing ceramic materials by x-ray and neutron small-angle scattering was published in the Journal of the American Ceramic Society (A. Allen, J. Am. Ceram. Soc., 88, 1367, 2005). The first computational model to correlate nanoscale chemical ordering, defects and properties in relaxor ferroelectrics, materials of choice for sonar and medical imaging transducers, was constructed, coupling first principles calculations and molecular dynamics simulations. Devices for calibrating force in commercial nanoindentors, instruments for measuring mechanical properties at the nanoscale used by thousands of researchers worldwide, have been developed jointly with scientists in the NIST Manufacturing Engineering Laboratory. These force calibration cells will be produced in collaboration with the major nanoindentor instrument manufacturers.

In a far-reaching effort to respond to the anticipated metrology and standards needs for next-generation advanced materials, particularly for nanotechnology applications, several new research efforts were initiated this year. In the area of CMOS technology, measurement methods are being developed and applied to evaluate thermal and electrical stability at interfaces in high-k dielectric gate stack structures and to characterize the electronic structure and chemical bonding in these structures at nanometer depth sensitivity. Metrology based on Raman spectroscopy and x-ray topography is under development to evaluate the stress state and defect structures in strained silicon layers for high-performance MOSFET devices.

Multifunctional oxide materials, wherein the functional response of one constituent phase/subsystem is generated by the response of another phase/subsystem to an external field, offer the potential for integrating electronic, magnetic and optical devices on a single chip. A combined experimental/theoretical modeling effort is underway to analyze the formation of self-assembled epitaxial nanostructures of multifunctional oxides, measure the functional properties at the nanoscale, and ultimately correlate the responses to the nanostructural architectures. Foundational pre-standards research on theoretical structural models for extracting film properties from $\mathrm{x}$-ray reflectometry measurements and on reference cantilevers for calibrating AFM force measurements has begun. A multi-year project was initiated to develop an in situ nanocalorimetry technique with adequate sensitivity to detect hydrogen desorption in hydrogen storage materials and interfacial reactions in multilayer structures in collaboration with world leaders in nanocalorimetry from the University of Illinois.

It is a pleasure to acknowledge the numerous prestigious honors bestowed upon the staff this year. Dr. Daniel Fischer was one of only twelve individuals to receive the coveted Arthur S. Flemming Award honoring outstanding Federal Government employees. Dr. Fischer was cited for his pioneering work in developing and utilizing a first-in-the-world facility for soft x-ray absorption spectroscopy that has enabled key scientific and technological advances in cutting-edge and emerging technologies of paramount importance to the Nation. For these exemplary achievements, Dr. Fischer was also awarded the Department of Commerce Gold Medal, the highest honorary award granted by the Secretary of Commerce.

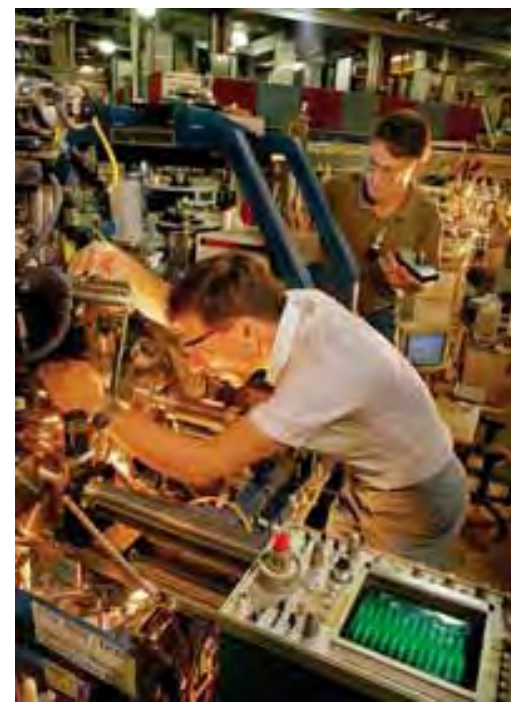

Dr. Daniel Fischer received the Arthur $S$. Flemming Award and the Department of Commerce Gold Medal award.

The Silver Medal, the second highest Department of Commerce honorary award, was given to a NIST team, including Dr. Douglas Smith, for their technical innovation that revolutionized the realization of the unit of force at the micro- and nanoscales. Dr. Vicky Karen and Dr. Alec Belsky (Technology Services) received the Bronze Award, the highest honorary recognition presented by the NIST Director, for their development and application of scientific algorithms and functional software embodied in the Inorganic Crystal Structure Database used for phase identification in commercial SEMs.

The esteemed NIST Edward Uhler Condon Award recognizing distinguished achievement in scientific and technical writing was awarded to Dr. Ronald Munro for his eloquent and systematic exposition of data evaluation as a scientific discipline. Nearly 1500 printed and 40,000 electronic copies of NIST Recommended Practice Guide "Data Evaluation Theory and Practice for Materials Properties" have been requested across the world, attesting to the broad appeal and applicability of the work.

We remain committed to providing high-quality metrology tools, standards, and data to support the development and implementation of advanced ceramic materials, components, and devices in the electronics, photonics, energy, and healthcare technology sectors.

Debra L. Kaiser

Chief, Ceramics Division 


\section{Materials Reliability Division}

\begin{abstract}
The Materials Reliability Division focuses on reliability issues in microelectronics, nanocharacterization, biomaterials metrology, and infrastructure reliability. Because we do not concentrate on a specific class of material, the division takes advantage of the specific expertise and complementary skills in the other MSEL Divisions by running many joint projects.
\end{abstract}

$\mathrm{T}$ he Materials Reliability Division's mission is to develop and disseminate measurement methods and standards enhancing the quality and reliability of materials for industry. Our work spans a wide range of materials, with a dimension span that extends from nanometer scale devices, carbon nanotubes, and single cells — to tall buildings, gas pipelines, and bridges.

\section{Recent Workshops}

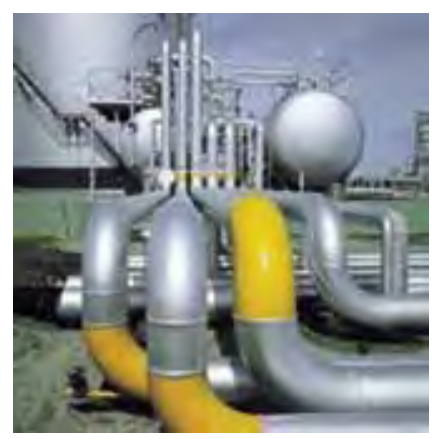

\section{Pipeline Coatings}

The proceedings of the 2004 workshop Coatings for Corrosion Protection: Offshore Oil and Gas Operations Facilities, Marine Pipeline, and Ship Structures has been mailed to the 150 participants and to key libraries around the country. This report is an important component of our support for the pipeline industry under the Pipeline Safety and Improvement Act of 2002 (HR 3609). Cosponsorship of the workshop (along with the Office of Pipeline Safety in DoT, DoI, and various academic and industry groups) helps drive improvements in safety in the pipeline industry.

\section{Acoustic Propagation}

With NIST's Center for Theoretical and Computational Materials Science (CTCMS), we sponsored a workshop on Computational Tools for Modeling Acoustic Propagation in Real-World Materials (CTAP) in August 2004. Researchers came together to discuss modeling requirements for non-destructive evaluation, medical ultrasound, underwater acoustics, geophysics, and atmospheric acoustics. Despite these very different uses of acoustics, the attendees faced similar modeling challenges and were interested in pursuing collaborative solutions with NIST's support.

\section{Carbon Nanotubes}

Along with other researchers in MSEL and CSTL, we co-organized the 2nd Joint NASA-NIST Workshop on Measurement Issues in Single Wall Carbon Nanotubes (SWCNTs) in January 2005. Over 80 leading researchers attended from industry, academia, and other government agencies to discuss two significant measurement problems: how to determine nanotube purity and how to ensure nanotubes are well dispersed for device processing. Based on information provided from attendees, we are currently drafting a NIST Recommended Practice Guide to help industry with these problems. The collected data will also serve as the basis for an upcoming IEEE nanotube measurement standard.

\section{Recognition}

Dr. Vinod K. Tewary was presented the Eric Reissner Medal at the annual International Conference on Computational and Experimental Engineering and Sciences meeting held July 2004 in Portugal. He received this award for sustained and significant contributions to mechanics at small-length scales.

Dr. Donna C. Hurley was named a Fellow of the Institute of Physics (U.K.) in recognition of her status in the physics community and her contribution to the Institute as a member of the Editorial Board of the Journal of Measurement Science \& Technology.

\section{Division Highlights by Focus Area}

\section{Materials for Micro- and Optoelectronics}

The International Technology Roadmap for Semiconductors recognizes the importance of metrology to the advancement of the industry and calls specifically for solutions to the near-term challenge (through 2009) of achieving necessary reliability. Our project in this area focuses on development of test and detection methodologies for mechanical reliability of dimensionally-constrained materials, as well as on learning more of the science behind the observed mechanical behavior.

\section{Highlight}

This year a key advance was made in the first known demonstration of time- and spatiallyresolved measurements of Joule heating in patterned metal interconnects, using alternating currents. The measurement is made with an atomic force microscope (AFM) that uses the probe tip as both a point-source heater and a resistive element in a Wheatstone bridge circuit.

The left figure shows a thermal AFM image depicting temperature differences measured at the surface of a non-passivated $\mathrm{Al}$ interconnect carrying an rms AC current density of $7 \mathrm{MA} / \mathrm{cm} 2$. A rapid decrease in temperature with distance away from the interconnect is apparent. The right figure shows 

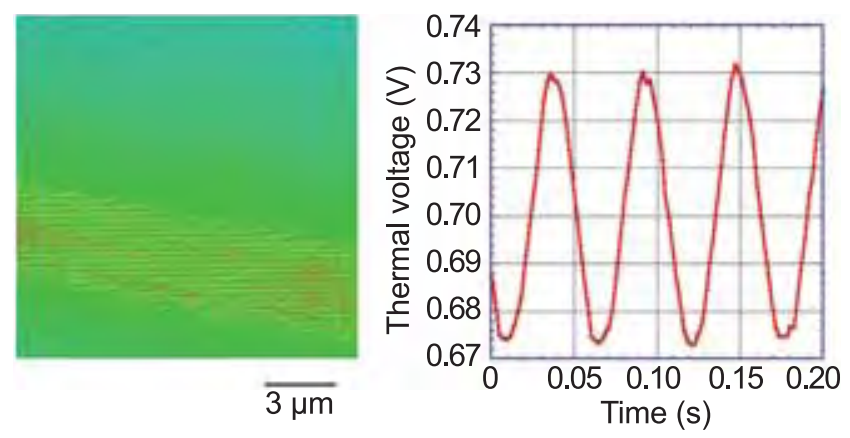

the temperature variation at one position of the AFM probe, over $0.2 \mathrm{~s}$, for a $10 \mathrm{~Hz}$ current; the oscillation is approximately $15^{\circ} \mathrm{C}$. These temperature measurements are used to quantify cyclic thermal strains applied to interconnects for the purpose of conducting quantitative thermal fatigue tests.

\section{Key Paper}

R.R. Keller, D.T. Read, and R. Mahajan, "Report of the Workshop on Reliability Issues in Nanomaterials," NIST Special Publication, in BERB (June 2005). This report establishes us as a leader in the field of reliability issues in nanomaterials.

\section{Nanocharacterization}

New measurement techniques play a key role in the development and commercialization of nanotechnology. Reliable manufacturing of nanoscale products demands vast improvements in our ability to measure material dimensions, characteristics, and structures. In particular, measurement of mechanical properties on the nanoscale is critical for fabricating structures with high-aspectratio features and ultra-thin and/or multilayer films.
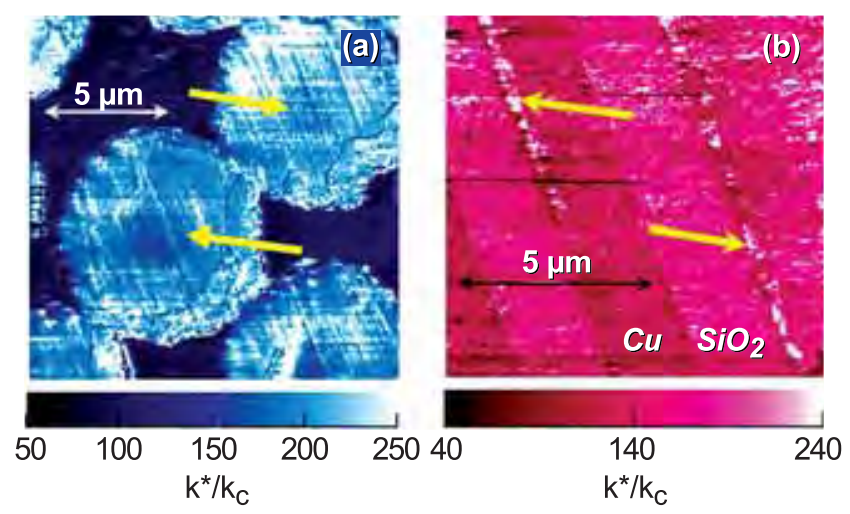

To investigate elasticity at the nanoscale, we are pursuing two techniques, atomic force acoustic microscopy (AFAM) and Brillouin light scattering (BLS), to measure Young's modulus and Poisson's ratio. This year, we successfully transitioned the AFAM technique from quantitative measurements at a single point to elastic modulus maps for an entire film (see figure for contact stiffness images of a composite and a microelectronic test structure). In addition, we used BLS to detect low-frequency vibrational modes in nanoimprinted polymeric lines, demonstrating the ability to measure elasticity of sub-100 nm features. Key papers on these subjects have been published in Nanotechnology, Advanced Engineering Materials, and Applied Physics Letters.

\section{Mechanical Behavior of Biological Materials}

The medical research community recognizes a need to understand the role that the mechanical behavior of biological materials play in normal and diseased tissues. We use a multi-scale approach to elucidate the mechanical behavior of individual cells and constructs of a single type of cell, and different cells in concert. We define mechanical behavior as the mechanical properties (e.g., stress, strain, modulus) and the measured response to mechanical stimulus (e.g., change in phenotype, proliferation, signaling production). At present, our focus is on measuring cell response by developing specialized bio-MEMS devices or with an optical trap, designing and developing specialized bioreactors for tissue-engineered constructs, and measuring mechanical properties of membrane-like tissue.

\section{Infrastructure}

The nation's infrastructure continues to age and is becoming more vulnerable to catastrophic failures, both intentional and unintentional. In response, we develop better measurement technology for determining a material's characteristics or

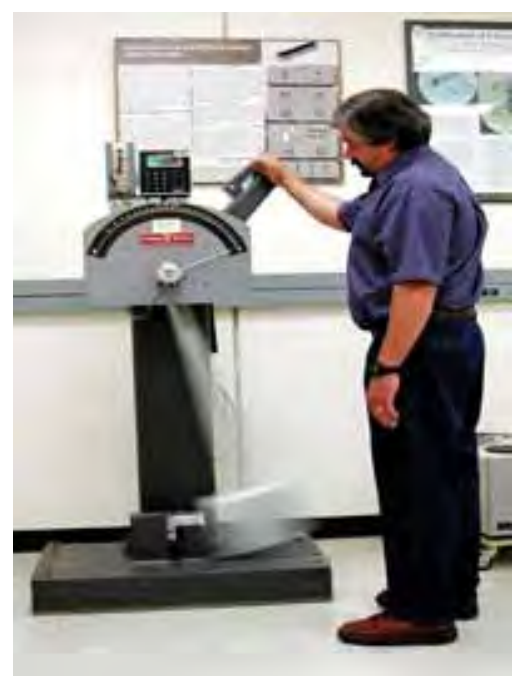
for assessing the sensitivity to failure. In FY05 we expanded our efforts on pipeline safety by developing fatigue data on new and field-damaged pipe, advancing the procedures for crack-tip opening angle (CTOA - a measure of the resistance to crack extension) testing, and holding workshops. The Charpy SRM program had over 850 customers in FY05, and we organized and contributed to an international workshop on Charpy test procedures. We finished measuring properties of steels used in the World Trade Center as part of the NIST-led study (reports released Summer 2005), and are looking at the issues that impede the use of fire-resistant steel.

Thomas A. Siewert

Acting Chief, Materials Reliability Division 


\section{Metallurgy Division}

The Metallurgy Division mission is to provide critical leadership in the development of measurement methods, standards, and fundamental understanding of materials behavior needed by U.S. industry. In extensive collaborations with industry and other agencies, this year we have focused on complex metallurgical issues in microelectronics, magnetics, automotive, and civil infrastructure areas.

Tn this summary, we describe highlights of the past 1 year, as well as demonstrate how the capabilities of the NIST Metallurgy Division are being used to solve problems important to the national economy and the materials metrology infrastructure.

\section{Establishing Priorities}

We examine a wide range of possible research topics and make choices for our research portfolio based on well-tested criteria: the match to the NIST and Division missions, the magnitude and immediacy of the need, whether our contribution is critical for success, the anticipated impact relative to our investment, our ability to respond in a timely fashion with high-quality output, and the opportunity to advance mission science, particularly in areas we believe are important for the future.

We establish our research priorities through extensive interaction with U.S. industry and other federal agencies, using a variety of methods, including roadmapping activities, workshops, technical meetings, standards committee participation, and consultation with scientific and technical leaders. A key aspect is translating our fundamental science into a form useful to our partners, integrating measurements, standards, software tools, and evaluated data as needed to solve industry needs.

We prefer to work in rapidly evolving technologies, where advances in measurement science are needed to understand the limitations on system behavior and, therefore, where our contributions are likely to have an impact on the course of technology. For NIST as a whole and the Metallurgy Division in particular, we are committed to having an impact on nanotechnology, homeland security, health care, and the materials infrastructure required for advances in information technology. Over the last two years, we have shifted substantial resources into the areas of nanomagnetics, nanomechanics, nanostructure fabrication, and critical infrastructure protection.

\section{Division Organization}

Carol Handwerker led the Division as Chief for the past nine years. Unfortunately for the division, she has now taken an academic position at Purdue University. Frank Gayle has assumed the position of Acting Chief.

This year we reorganized into four technical groups, each consisting of about 10 staff members and a like number of guest researchers.

- The Thin Film and Nanostructure Fabrication Group performs cutting edge research in fabrication techniques that range from electrochemical processing to vapor-liquid-solid growth of nanowires.

- The Thermodynamics and Kinetics Group develops new theory, simulation methods, and data for predicting and controlling phase transformations during materials fabrication processes.

- The Magnetic Materials Group develops new materials and metrology that allow magnetic devices and sensors to be created at smaller size scales and with greater sensitivities.

- The Materials Performance Group develops new theory and measurements for characterizing the mechanical behavior of materials, with applications as varied as sheet metal forming and critical infrastructure protection.

\section{Division Highlights}

By virtue of the interdisciplinary nature of materials problems, teams are formed across the Division, MSEL, and NIST, and, in most cases, with external partners in order to meet our project goals. A few highlights of the past year are presented here, grouped by focus areas.

\section{Safety and Reliability}

The nation's infrastructure continues to age and is ever more vulnerable to catastrophic failures, whether accidental, intentional, or due to forces of nature. To address infrastructure vulnerability issues, we have started a number of projects, several in close collaboration with the Materials Reliability Division (MRD).

This year, NIST completed the three-year Investigation of the World Trade Center Disaster. A critical aspect of the investigation was the metallurgical analysis of the recovered structural steel performed by the Metallurgy Division with MRD. We issued six reports totaling 1500 pages, and we continue to work on issues impeding the use of fire-resistant steel.

In FY05 we expanded our efforts on pipeline safety in a project working with DoT's Office of Pipeline 
Safety and DoE, with MRD focusing on crack arrest behavior, and Metallurgy addressing corrosion issues, including topics arising at a Metallurgy-organized Pipeline Coatings Workshop held at NIST.

\section{Advanced Manufacturing Methods}

A software tool, FiPy, for simulating phase transformations was released to the public. FiPy gives materials scientists, physicists, and chemists the ability to simulate phase transformations using state-of-the-art, sophisticated numerical techniques for partial differential equations that allow dramatically faster solutions to be found over larger physical domains and for longer elapsed simulation times.

NUMISHEET, a triennial international conference on simulation techniques for sheet metal forming, was held in Detroit in August 2005. Division scientists served on two organizing committees, gave a plenary lecture, and several additional invited talks. An integral part of this conference is a round-robin simulation exercise where modeling groups try to predict the final sample shape from a prototypical forming operation. MSEL played the key role in providing new benchmark data using its unique capabilities for in situ and ex situ stress measurement. Through thickness residual stresses were measured at the NCNR, and surface stresses in a sheet under load in a die were measured using a newly developed X-ray stress measurement system integrated into our metal forming machine.

\section{Nanometrology}

A new magnetoresistance (MR) effect discovered this year by scientists in the Metallurgy Division is antisymmetric with respect to magnetic field. This new MR effect is due to the presence of circulating currents created around domain walls when the magnetization vector, current direction, and domain wall are mutually perpendicular. This effect may provide device designers with much more flexibility in designing magnetic switches and memory.

A new strategy for selecting appropriate catalytic metals for vapor-liquid-solid growth of $\mathrm{ZnO}$ nanowires with specific semiconducting properties was developed by combining thermodynamic information from phase diagrams with a high-throughput (combinatorial) approach recently demonstrated by the MSEL Metallurgy Division for metallizations to wide-band-gap semiconductors.

\section{Materials for Microelectronics}

Spontaneous formation of tin whiskers on thin films of lead-free solder is an enormous reliability issue in the conversion of microelectronics to lead-free solders.
The mechanism responsible for the nucleation and growth of Sn whiskers is a matter of considerable debate. Our research this year has eliminated several of the possible mechanisms and points to critical experiments needed to distinguish among some of the remaining possible mechanisms. Knowing the mechanism for whisker formation will be key to the control and hopefully suppression of Sn whisker formation.

Our past work provided tools to chip manufacturers for on-chip copper interconnect development for next generation chips. We have moved successfully into several new and exciting areas: we have included the impact of leveling additives in our Curvature Enhanced Accelerator Coverage (CEAC) model — these are used industrially to control overfill bump formation; we have demonstrated gold superfill; we have completed a thorough assessment of the issues relevant to successful wetting and superfill of copper on ruthenium barriers that is already being requested by industry and will be key to successful implementation in industry, should it occur; we have achieved seedless superfill on osmium barriers, potentially superior to ruthenium as a diffusion barrier, a key issue for barriers for seedless superfill; we have started new projects on seedless superfill and experimental studies of leveling agents.

\section{Recognition}

Bill Egelhoff became a NIST Fellow, the highest scientific and technical position at NIST.

Lyle Levine and Richard Fields (retired) were part of a team of scientists that won the Allen V. Astin Measurement Science Award for outstanding achievement in the advancement of measurement science. This award recognized the development of techniques to measure stress-strain relationships of materials under high heating-rate, high strain-rate conditions.

The Metallurgy Division was recognized by Science News "News of the Year" in December 2004 for two breakthrough developments:

- Bob Shull led a team which found that a small amount of iron added to $\mathrm{Gd}_{5} \mathrm{Ge}_{2} \mathrm{Si}_{2}$ resulted in a significantly improved refrigerant, pointing the way to potential commonplace magnetic refrigeration.

- Jim Warren (Metallurgy) and Jack Douglas (Polymers) were recognized for showing a duality between kinetic and static effects controlling microstructure during solidification.

Frank W. Gayle

Acting Chief, Metallurgy Division 


\section{Polymers Division}

$\mathrm{T}$ his report marks an excellent year for the Polymers Division in terms of impacts not only in technical areas, but also in professional impact for our staff. This year, Joseph Antonucci of our Biomaterials Group was awarded the FLC Technology Transfer Award for his research, patenting, and technology transfer activities resulting in commercialization of restorative dental resins; Michael Fasolka of our Multivariant Measurement Methods Group received the Presidential Early Career Award for Scientists and Engineers (PECASE) for his work in nanostructured polymer films and scanned probe techniques; and Wen-li Wu was named a NIST Fellow for his high-impact advances in measurement methods to assist industry, developments in the fundamentals of scattering, and significant scientific insights in polymer physics.

This year, we are pleased to present highlights in areas ranging from nanomanufacturing and nanofabrication to organic electronics to combinatorial methods to standards test methods. Further information on these technical highlights is available on the Polymers Division's website at www.nist.gov/polymers.

\section{Chaotic Mixing in Microfluidic Channel Flows}

Realization of nanomanufacturing requires the ability to efficiently mix disparate liquids, which is difficult to achieve in small-scale microfluidic devices. This requires the development and use of new mixing technologies. A promising candidate for enhancing mixing is chaotic flow, where the flow-streamlines repeatedly cross themselves resulting in mixing that is orders of magnitude more efficient than diffusion. We determined precisely how to generate a chaotic flow and developed metrics to assess the degree of chaos.

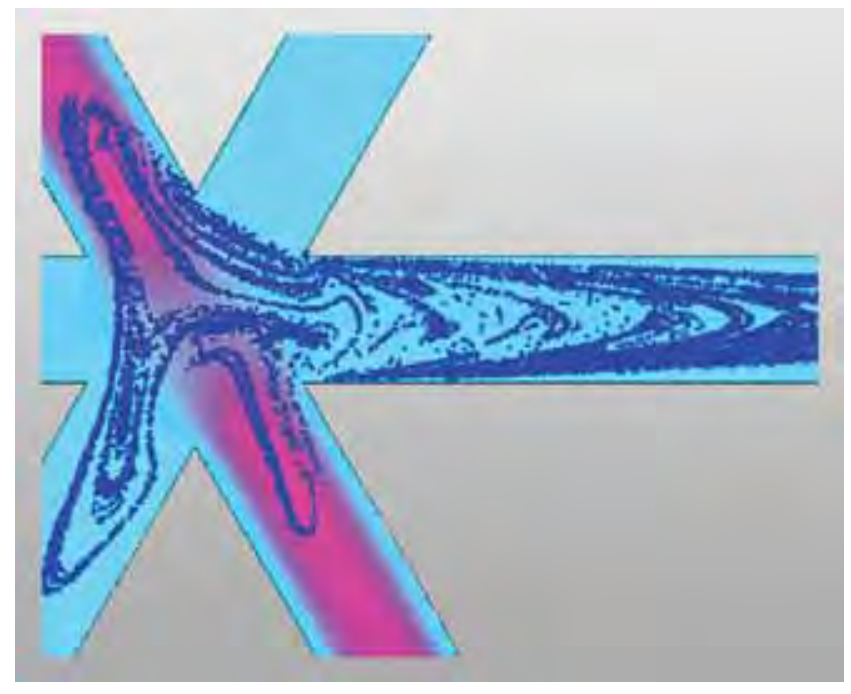

The “crossing-streamline” principle was used to study the generation of chaotic mixing in intersecting channels driven by oscillatory flow boundary conditions, which serve as the source of the necessary temporal variations. Our advances represent new paradigms in nanomanufacturing.

\section{Metrology for Nanoimprint Lithography}

Nanoimprint lithography (NIL) is an exciting technology for cost-effective routine fabrication of polymers and other soft materials into 3D nanoscale structures by physically molding them with a hard master. Nanoimprinted structures with dimensions $<10 \mathrm{~nm}$ have been achieved without the expensive, complex infrastructure required of next generation photolithography. Future advancements in NIL, however, require new measurements of dimensions with sub-nm precision, the characterization of structures with complex shapes, and investigations of the properties of new and unique nanostructured materials. The importance of these metrology needs were established through a panel discussion consisting of 7 experts and 300 audience participants, led by Christopher Soles from the Polymers Division, at the 2005 SPIE Microlithography Conference in Santa Clara, CA. The panel featured representatives from leading nanoimprint vendors, universities, semiconductor companies, and nanotechnology industries.

\section{Influence of Interfacial Structure of Organic Semiconductors on Device Performance}

Organic electronic devices are increasingly incorporated into electronics packaging and are projected to revolutionize integrated circuits through new applications taking advantage of low-cost, high-volume manufacturing, nontraditional substrates, and designed functionality. Adoption of these devices will be advanced by developing an integrated and interdisciplinary suite of measurement methods correlating device performance with structure, properties, and chemistry of critical materials and interfaces.

Near-edge $\mathrm{x}$-ray absorption fine structure (NEXAFS) spectroscopy was used for investigating the electronic structure, chemistry, and orientation of several organic electronic molecules near a supporting substrate. In collaboration with the University of California-Berkeley, NEXAFS spectroscopy was applied to successfully quantify the simultaneous chemical conversion, molecular ordering and defect formation 


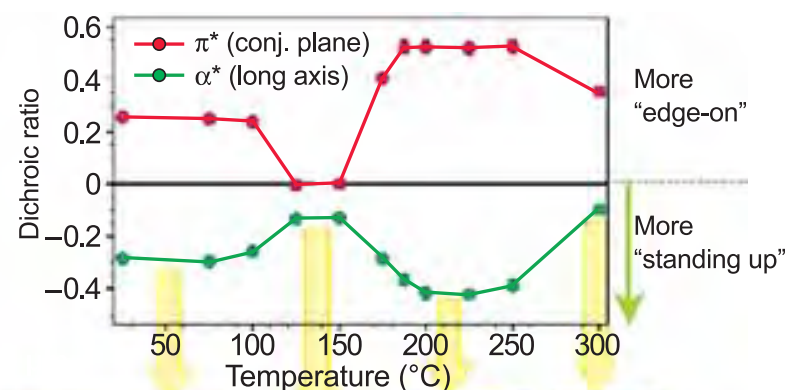

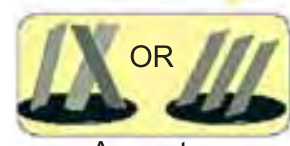

As cast weak orientation

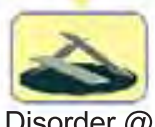

Disorder @ onset

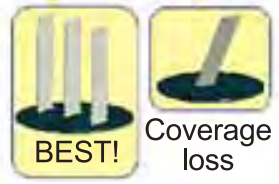

of soluble oligothiophene precursor films for application in organic field effect transistors (OFETs). Variations in field-effect hole mobility on thermal processing correlated directly with the orientation and distribution of molecules within (3 to 20) nm thick films.

\section{Gradient Libraries of Surface-Grafted Polymers}

Layers of grafted polymers provide the means for the physically robust, chemically versatile surface functionalization required for advanced applications, such as friction management in MEMS, adhesion promotion for coatings, protein adsorption control in biomaterials, and environmentally responsive surfaces for sensors. However, while recent advances in controlled polymerization enable grafted polymers that exhibit many types of architecture and composition, identifying the optimal grafted system for a given application can be difficult, time consuming, and expensive.

In response, the NIST Combinatorial Methods Center has developed tools for probing the optimal molecularto micro-scale properties of grafted polymer systems. These methods employ microfluidic technology to deliver tailored mixtures and sequences of monomers to an initiator-functionalized surface. The resulting grafted polymer libraries exhibit gradual, systematic changes in composition, chain length, and architecture. Gradients of grafted block copolymers prepared via these techniques reveal composition regimes that "switch" their surface properties in response to solvent exposure. Fabrication of layers, e.g., of grafted tapered copolymers exhibiting a gradual change in composition along the polymer chain, have also been demonstrated. Moreover, our unique ability to prepare statistical copolymer composition gradients provides comprehensive maps of complex surface chemistry, which were previously impossible.

\section{Polymer Grafting Density, Protein Adsorption, and Cellular Response}

A combinatorial library consisting of a cell-adhesive protein-coated poly(2-hydroxyethyl methacrylate) (poly(HEMA)) gradient with variable grafting densities was developed to investigate cell adhesion. Fibronectin (FN), a well-characterized extracellular matrix protein, was selected as a model protein to study the effect of poly(HEMA) grafting density on cell adhesion, protein adsorption, and cellular response. The $2 \mathrm{D}$ conformational gradient varied from low- to high-graft density with the polymer chain structure changing from mushroom- to brush-like regimes, respectively, with the FN positioned between poly(HEMA) chains. By varying the chemistry, morphology, and functionality of this film, the number of adherent cells and their corresponding shapes varied significantly. Cell adhesion and spreading were used to evaluate the effect of poly(HEMA) grafting density on cellular response. A maximized cell adhesion and spreading response was found at low grafting density/high FN density, and little cell adhesion and spreading was found at high grafting density/low FN density. The experimental results of protein adsorption, cell adhesion, and spreading were consistent between gradient samples and uniform samples, strongly indicating that gradient preparation technology can be used for combinatorial studies of surface-protein-cells interactions.

\section{ASTM Test Method for Mass Spectrometry of Polymers}

Charles Guttman of the Polymers Division initiated and led Technical Working Activity 28 of the Versailles Project on Advanced Materials and Standards (VAMAS) to developed an ASTM Standard Test Method to determine the molecular mass distribution of polystyrene using matrix-assisted laser desorption/ionization mass spectrometry (MALDI MS). The method uses data and protocols developed in a NIST-sponsored interlaboratory comparison organized with the help of the American Society for Mass Spectrometry, a complete description of which has been published in Analytical Chemistry. This method covers the determination of molecular mass averages and the distribution of molecular masses for linear atactic polystyrene of narrow molecular mass distribution ranging in molecular masses from $(2,000$ to 35,000$) \mathrm{g} / \mathrm{mol}$, and it provides detailed procedures for instrument calibration and sample preparation, as well as details for measuring the samples on a mass spectrometer.

Eric J. Amis

Chief, Polymers Division

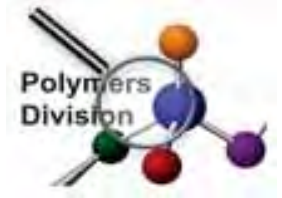




\section{NIST Center for Neutron Research}

$\mathrm{T}_{\mathrm{is}}^{\mathrm{h}}$ The NIST Center for Neutron Research (NCNR) is the nation's leading neutron facility. This year more than 2000 research participants from all areas of the country, from industry, academia, and government used the facility for measurements. These researchers primarily gain access through a peer-reviewed proposal system with beam time allocated by a Program Advisory Committee twice a year. The NCNR also supports important NIST research programs in nanotechnology, materials science, chemistry, physics, and biotechnology.

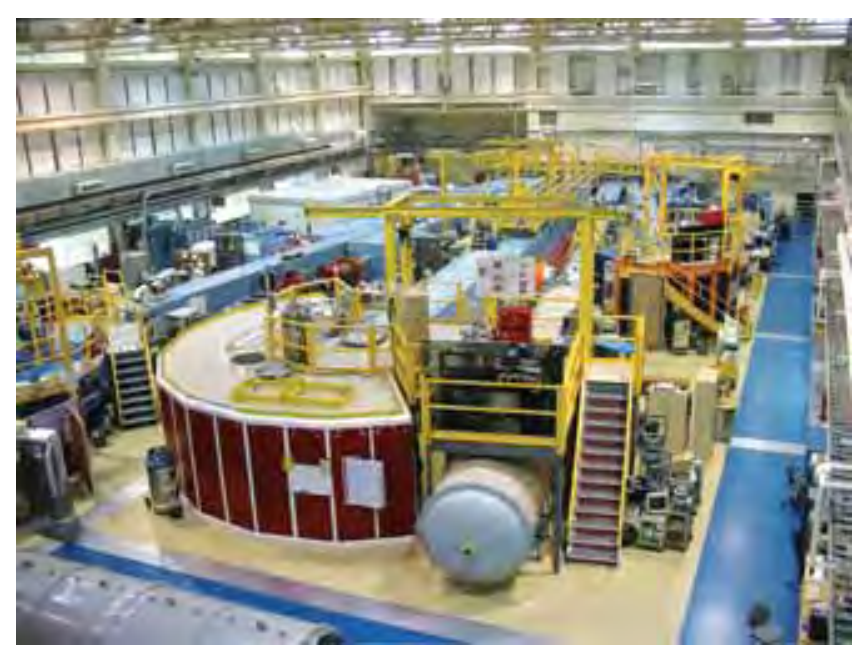

The NCNR source provides intense beams of neutrons to nearly thirty experimental stations. In addition to a thermal neutron $\mathrm{D}_{2} \mathrm{O}$ moderator, the NCNR has a large area liquid hydrogen moderator, or cold source, that provides intense neutron beams to the only cold neutron facility operating in the U.S. The operation of the facility over the past twelve months has been outstanding. This year, the NCNR ran as scheduled, completing nearly 200 beam days of operation and a major outage for maintenance and upgrade of the source and to install new instruments. The cold source and beam delivery systems also continue to provide highly reliable service.

Partnerships are integral to the success of the NCNR. The Center for High Resolution Neutron Scattering (CHRNS), a partnership with the National Science Foundation that funds the operation of six world-class neutron scattering instruments, was renewed for another five years. CHRNS serves more than 500 users who produce nearly 100 publications annually.

The Cold Neutrons for Biology and Technology (CNBT) consortium is dedicated to studies of biological membranes. The centerpiece of CNBT, funded by the National Institutes of Health, is the Advanced Neutron Diffractometer/Reflectometer (AND/R).

The ExxonMobil Research and Engineering Company continued its long-standing partnership with the NCNR through their participation in the operation, maintenance, and research at the NG-7 30 m SANS instrument. They use neutron scattering techniques to deepen their understanding of ExxonMobil's products and processes, so as to improve customer service and the return on shareholders' investment.

The Department of Energy (DOE) maintains several programs at the NCNR. Brookhaven National Laboratory directly partners with NIST through the BNL-NIST Scientific Alliance. In the last year, the NCNR joined the Metal-Hydride and Carbon-Based Materials Centers of Excellence, sponsored by DOE's Office of Energy Efficiency and Renewable Energy, to develop materials for hydrogen storage. The Center for Food Safety and Applied Nutrition, U.S. Food and Drug Administration (FDA) maintains a neutron activation analysis (NAA) facility at the NCNR that provides analytical support for FDA programs. The Smithsonian Institution's Nuclear Laboratory for Archeological Research has had a productive 28-year partnership with NCNR, during which time it has analyzed over 30,000 archaeological artifacts by NAA.

The NCNR has continued to upgrade and expand its measurement capabilities. These instrument developments remain a priority since improvements in capabilities lead directly to the long-term scientific success of the facility. The NCNR is in the advanced stages of manufacturing and installation of two new crystal spectrometers that will bring enormous improvements in capability for inelastic neutron measurements to the NCNR and open up new research opportunities. The new BT-7 thermal triple-axis spectrometer will be ready for users in the next year. The other new instrument, the Multi-Analyzer Crystal Spectrometer (MACS), is a partnership between NIST, the National Science Foundation, and The Johns Hopkins University. It will also begin commissioning in the next year. Substantial progress has also been achieved on an additional thermal triple-axis spectrometer to be installed at BT-9 and on the new time-resolved SANS capability that will allow researchers to access kinetic phenomena on time scales of $50 \mathrm{~ms}$ to $100 \mathrm{~ms}$ using stroboscopic techniques.

The more than 300 publications resulting from work done at the NCNR during the past year include 
numerous notable scientific achievements. The following are two representative research highlights.

Developing safe, cost-effective, and practical means of storing hydrogen is crucial for the advancement of fuel-cell technologies. The NCNR's Taner Yildirim, in collaboration with Prof. Salim Ciraci, has used first-principles calculations to show that carbon nanotubes "decorated" with titanium or other transition metals can bond to up to four hydrogen molecules per Ti. This corresponds to nearly 8 percent of the weight of "decorated" carbon nanotubes, one-third better than the 6 percent minimum storage-capacity requirement set by the Freedom Car Research Partnership involving the DOE and the nation's "Big 3" automakers. These bound hydrogen molecules are readily relinquished when the system is gently heated. Such reversible desorption is another requirement for practical hydrogen storage. Thus, these findings suggest a method of engineering new nanostructures for efficient, high-capacity hydrogen storage. This work, which appeared in Physical Review Letters, has been featured in a wide variety of forums including Materials Today, Fuel Cell Review, and C\&E News.

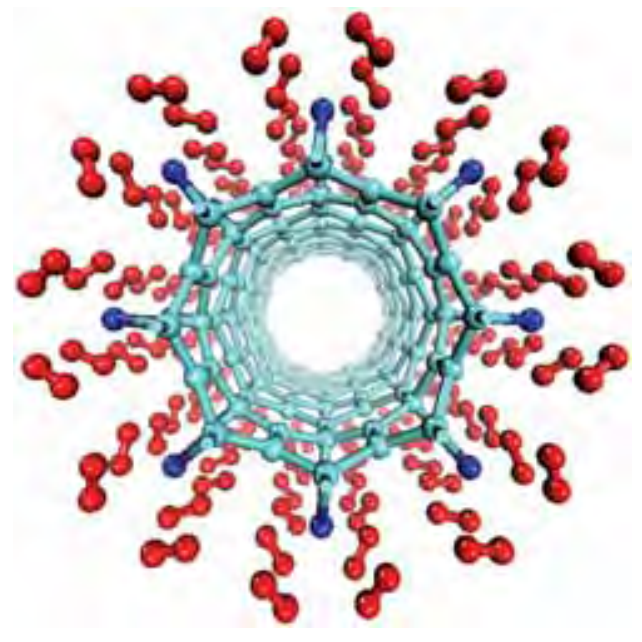

Wormlike micellar solutions formed by self-assembled surfactants exhibit rheological properties such as shear thinning and suppression of turbulent flow that are related to the flow-induced changes in their structural conformations or orientations. These flow properties are important in applications such as thickeners, drag reducers and flow improvers in the food and cosmetics industries. A collaboration between the University of Delaware and the NCNR combined SANS with a novel shear cell to measure the microstructure of a shear-induced phase separating wormlike micellar solution. The results show that both concentration fluctuations and gradients in segmental alignment occur, and they link shear banding in wormlike micellar solutions to a shear-induced phase separation.
This year, several prestigious honors were bestowed upon the users and staff of the NCNR. Charles F. Majkrzak was named the recipient of the 2006 Warren Award for Diffraction Physics. Dr. Majkrzak was cited "for his seminal contributions to the development of neutron reflectivity and for his pioneering work in the exploration of many issues

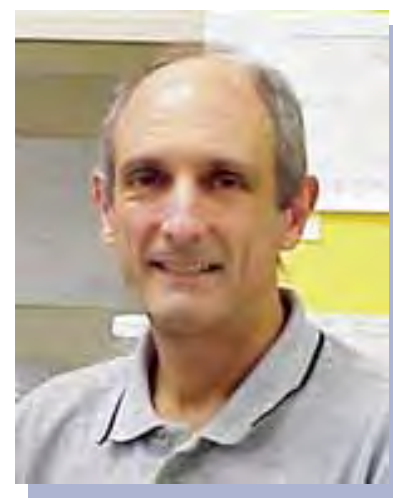

Dr. Charles F. Majkrzak in interface science using this technique.” An NCNR team including Charles Majkrzak, Susan Krueger, Joseph Dura, and Donald Pierce was awarded a Department of Commerce Silver Medal for their scientific and engineering leadership in establishing CNBT at the NCNR.

Taner Yildirim received a Bronze Award for theoretical research which showed how the physical and chemical properties of carbon nanotubes can be tuned with pressure. Paul Brand and Henry Prask of the NCNR, along with Richard J. Fields of the Metallurgy Division, were awarded the NIST Jacob Rabinow Applied Research Award for developing the nation's best capabilities for measuring residual stress depth profiles and texture in metals, ceramics, and composites. Brian Toby was elected Fellow of the International Centre for Diffraction Data (ICDD) for his contributions to the standardization of data formats and the creation of tools to use these formats efficiently. Vanessa Peterson received the 2005 Brunauer Award from the Cements Division of the American Ceramic Society for the best paper on the topic of cements published by the American Ceramic Society during the previous year.

Two long-time NCNR users won awards for work in which neutron scattering results played a major part. Dr. Frank Bates, Distinguished McKnight University Professor and Head of Chemical Engineering and Materials Science at the University of Minnesota, was presented the Turnbull Lecturer Award "for pioneering contributions to the fundamental understanding of structure and properties of complex polymeric materials, particularly block copolymers and polymeric vesicles, coupled with outstanding lecturing, writing, teaching, and educational leadership.” Dr. Thomas Russell, Distinguished Professor of the Polymer Science \& Engineering Department of the University of Massachusetts Amherst was awarded the 2005 Polymer Physics Prize "for his pioneering research and fundamental elucidation of the surface and interfacial behavior of polymers."

Patrick D. Gallagher

Director, NIST Center for Neutron Research 


\section{Nanometrology}

Nanotechnology will revolutionize and possibly revitalize many industries, leading to new and improved products based on materials having at least one dimension less than $100 \mathrm{~nm}$. The federal government's role in realizing the full potential of nanotechnology is coordinated through the National Nanotechnology Initiative (NNI), a multi-agency, multi-disciplinary program that supports research and development, invests in a balanced infrastructure, and promotes education, knowledge diffusion, and commercialization in all aspects of nanoscale science, engineering, and technology. NIST's unique and critical contribution to the NNI is nanometrology, defined as the science of measurement and/or a system of measures for nanoscale structures and systems. NIST nanometrology efforts focus on developing the measurement infrastructure measurements, data, and standards - essential to advancing nanotechnology commercialization. This work provides the requisite metrology tools and techniques and transfers enabling measurement capabilities to the appropriate communities.

MSEL plays a vital role in nanometrology work at NIST with efforts in four of the seven NNI Program Component Areas - Instrumentation Research, Metrology and Standards for Nanotechnology; Nanomaterials; Nanomanufacturing; and Fundamental Nanoscale Phenomena and Processes. Innovative projects across MSEL are defining and addressing the forefront research issues in these areas.

\section{Instrumentation Research, Metrology and Standards for Nanotechnology}

$R \& D$ pertaining to the tools needed to advance nanotechnology research and commercialization. The design, development, and fabrication of nanodevices will require nanomechanical measurements that are rapid, accurate, predictive, well-understood and representative of a device or system's environment in real time. MSEL is addressing this need by developing instrumentation, methodology, reference specimens and multi-scale modeling approaches to quantitatively measure mechanical properties such as modulus, strength, adhesion, and friction at nanometer-length scales. This year, novel instruments for measuring adhesion and friction forces between surfaces and nanoparticles were developed jointly with industrial partners. Quantitative maps of elastic modulus were obtained by innovative methodologies based on atomic force microscopy and strain-induced elastic buckling instability. To address the need for quantifying measurements made with widely-used commercial nanoindentors and scanned probe microscopy instruments, MSEL is developing reference specimens and SI-traceable force calibration methodology.

\section{Nanomaterials}

Research aimed at discovery of novel nanoscale and nanostructured materials and at a comprehensive understanding of the properties of nanomaterials. Among the many classes of nanomaterials, nanotubes have received great attention due to their remarkable physical properties relevant to many applications. In response to needs expressed by industry and other federal agencies, MSEL has embarked on a new effort to develop a suite of metrologies and standards aimed at characterizing key structural features and processing variables of carbon nanotubes. These include dispersion, fractionation, orientation, alignment, and manipulation of individual single-walled nanotubes, all critical to establishing efficient bulk processing schemes to meet the imminent high demand for carbon nanotubes.

\section{Nanomanufacturing}

$R \& D$ aimed at enabling scaled-up, reliable, cost-effective manufacture of nanoscale materials, structures, devices, and systems. Nanoimprint lithography (NIL) is rapidly emerging as a viable high-throughput technique for producing robust structures with a patterning resolution better than $10 \mathrm{~nm}$. MSEL is developing metrologies that are crucial to advancing NIL as an industrial patterning technology for the electronics, optics, and biotechnology industries. The current focus is on characterizing shape and the fidelity of pattern transfer, two key factors in achieving widespread commercial application of NIL.

\section{Fundamental Nanoscale Phenomena and Processes}

Discovery and development of fundamental knowledge pertaining to new phenomena in the physical, biological, and engineering sciences that occur at the nanoscale. The magnetic data storage industry needs the ability to measure and control magnetization on nanometer length scales and nanosecond time scales to meet increasing demands for reduced size and increased speed of devices. MSEL is developing measurement techniques to elucidate the fundamental mechanisms of spin dynamics and damping in magnetic thin films. Work this year has focused on measurements of the effects of interfaces and interface roughness on magnetization dynamics and magnetic characterization of edges in magnetic devices.

Through these and other research activities, MSEL is maintaining its committed leadership in developing the measurement infrastructure for current and future nanotechnology-based applications.

Contact: Debra L. Kaiser (Ceramics Division) 


\section{Mechanical Metrology for Small-Scale Structures}

\begin{abstract}
Industrial trends toward miniaturization require quantitative mechanical property data for design, development, and fabrication of modern small-scale devices. Developments in disruptive technologies require engineering materials data for structures and architectures at multiple (nano to macro) length scales. Accordingly, we are designing and developing mechanical testing configurations from small-scale in-situ structures for localized measurements of fracture and deformation behavior of materials and interfaces.
\end{abstract}

\section{Edwin R. Fuller, Jr. and Douglas T. Smith}

$\mathrm{T}$ This project aims to: (i) measure mechanical properties of microstructures for myriad industrial and biological systems that cannot be fabricated in bulk samples; (ii) study small-scale phenomena, which may be controlled by surface effects (e.g., the influence of surface stresses on crack nucleation and extension); and (iii) obtain quantitative mechanical property data of materials and interfaces for designing small-scale structures and components and for assessing their mechanical reliability. Well-characterized testing configurations are being designed and developed for measurements of strength and crack extension of small-scale structures and interfaces. We are pursuing four tasks: (i) configuration design, optimization, and characterization via finite-element analyses; (ii) specimen fabrication; (iii) mechanical testing and fracture analysis (fractography); and (iv) length and force metrology. In addition to work in the Ceramics Division (tasks i, iii, and iv), two collaborations were established in the fabrication task (ii): one with James A. Beall of the Quantum Electrical Metrology Division in NIST Boulder, and one with Northwestern University.

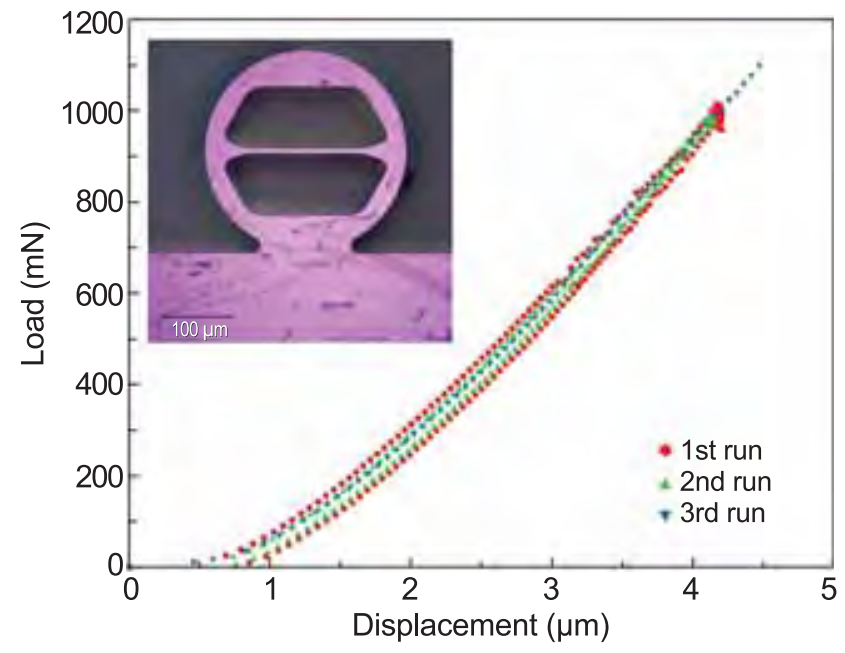

Figure 1: Repeatability for multiple loading of a single specimen.
Significant progress has been made in the design of a compressively loaded test configuration with a well-defined, tensile gage section. The inset of Figure 1 shows one such specimen fabricated by James Beall using deep reactive ion etching (DRIE) of a silicon wafer. When a load is applied to the top of this theta-like geometry, a uniform uniaxial tensile stress develops in the middle gauge section. Finite element analysis gives (horizontal) gauge section stress and strain as functions of the applied load and load-point displacement, respectively. Dimensionless calibration factors have been obtained for several specimen and fillet geometries, and as a function of the gauge width.

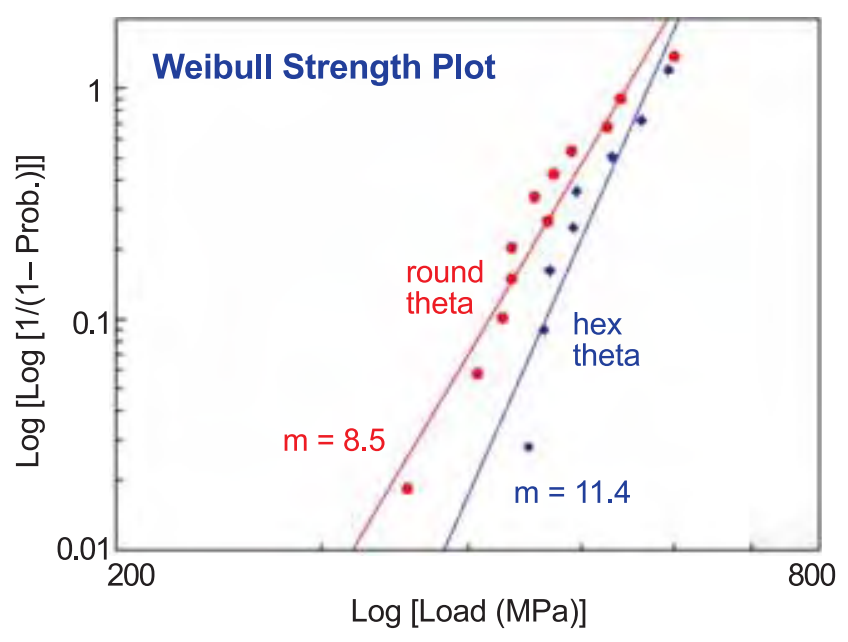

Figure 2: Weibull plots for round and hexagonal configurations.

Specimens are tested using a depth-sensing nanoindenter as a universal testing machine, thereby giving a continuous record of applied load and load-point displacement. Repeatability is illustrated in Figure 1. Strength data for DRIE silicon, Figure 2, suggest that differences for round and hexagonal specimen configurations are not significant.

To extend this technique to a wider variety of material systems, focused-ion-beam (FIB) milling is being explored in collaboration with Northwestern University. Hexagonal theta specimens fabricated from a lamellar directionally solidified eutectic of $\mathrm{Ni}_{0.5} \mathrm{Co}_{0.5} \mathrm{O}$ and $\mathrm{ZrO}_{2}$ are approximately $1 / 15$ the size of the silicon specimens.

\section{Contributors and Collaborators}

D. Xiang, G.D. Quinn, D.L. Henann (Ceramics Division, NIST); J.A. Beall (Quantum Electrical Metrology Division, NIST); N. Alem, V.P. Dravid (Northwestern University) 


\section{Nanomechanics: Atomistics in Modeling and Experiments}

\begin{abstract}
Nanoscale mechanical behavior (including failure) is inherently difficult to measure accurately, and existing modeling tools are only qualitative at best. We are developing modeling techniques that provide quantitative predictions and validating these results experimentally.
\end{abstract}

\section{Lyle E. Levine, Douglas T. Smith, and Anne M. Chaka (838)}

$\mathrm{M}$ echanics at the nanoscale is inherently difficult to model accurately. Finite element models (FEM) can effectively capture the elastic behavior of macroscopic structures but include no accurate failure criteria, since this depends upon atomic-scale behavior. Classical atomistic simulations can handle millions to billions of atoms, enough to model such events, but these potentials become inaccurate for large strains and cannot effectively handle chemistry. Quantum-mechanics-based simulations using density functional theory (DFT) are extremely accurate and handle the chemistry exactly, but such simulations are so CPU-intensive that they can handle only a few hundred atoms. A combination of all three modeling techniques is required to accurately model device behavior at the nanoscale.

Over the past year, we have been developing quantitative multiscale modeling techniques for quasistatic applications. At the macroscale, FEM is used to simulate the elastic behavior of a nanomechanical system. Figure 1 shows an example in which a $<111>$ $\mathrm{Al}$ crystal is indented by a nanoindenter. The shape of the nanoindenter was obtained using an atomic force microscope with closed-loop displacement control. The fine central mesh has nodes on fcc atom positions. The figure shows the resulting von Mises stress distribution (in cross-section) after indenting $1.5 \mathrm{~nm}$.

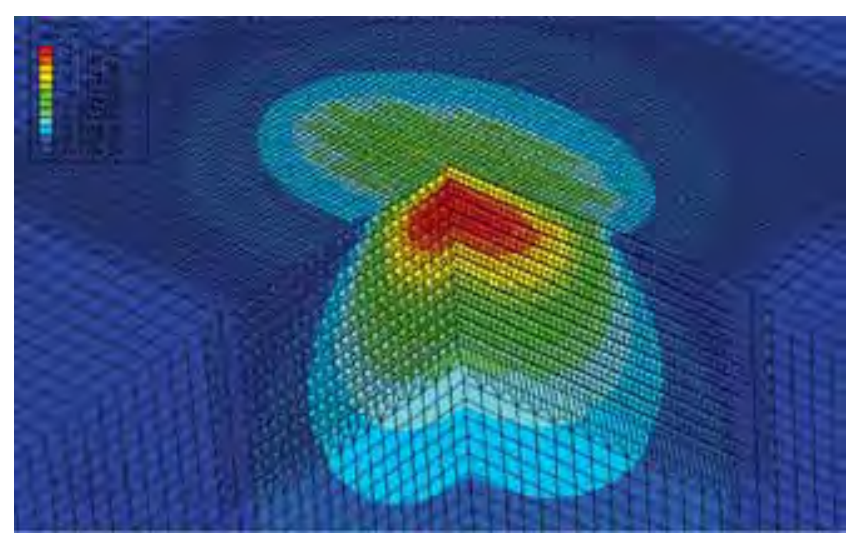

Figure 1: FEM model of a rigid nanoindenter indenting $a<111>$ aluminum single crystal to a depth of $1.5 \mathrm{~nm}$.
The use of classical potentials in a large simulation cell allows us to propagate long-range stresses to the critical regions where bond distortions are large or where chemistry effects need to be explored. In these critical regions, we embed a DFT simulation. The critical region is relaxed iteratively using DFT, and the classical cell is relaxed using a molecular dynamics algorithm.

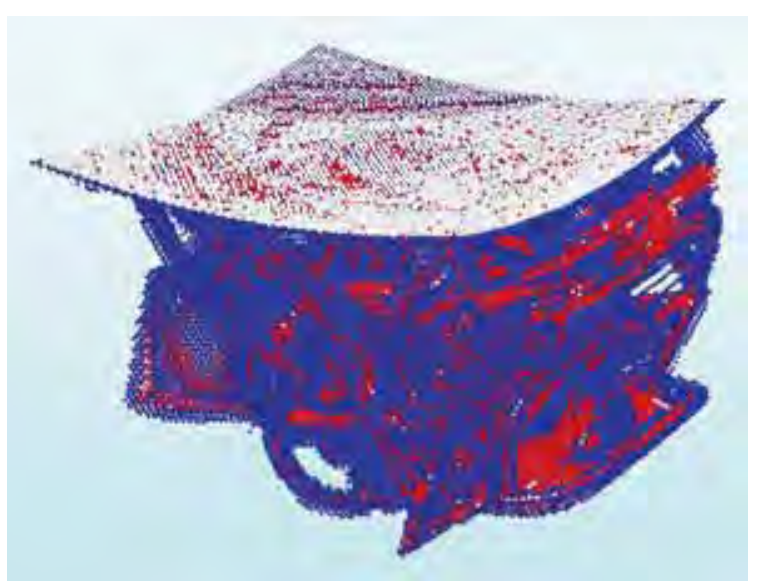

Figure 2: EAM simulation showing dislocations produced by nanoindentation of $<111>$ Al to a depth of $4.5 \mathrm{~nm}$.

Figure 2 shows a classical atomistic simulation using the embedded atom method (EAM). Initial atom positions and boundary conditions came from a FEM simulation. The subsurface structure is composed of dislocations that nucleated from a single event.

After an initial dislocation nucleation event, nanoindentation progresses through the complex evolution of dislocation structures. For example, the raised lip around an indent is produced by large numbers of dislocations exiting the surface. We are modeling the early stages of this process using 3D dislocation dynamics, assuming a random distribution of dislocation sources.

Finally, connection to experimental measurements requires careful force calibration of the indenter and calibrated atomic force microscopy measurements of the indenter tip. We are setting up to grow bulk single-crystal copper samples, which will be cut and polished using non-contact chemical methods to minimize the dislocation density. We are also developing SI-traceable calibration methodology for nanoindenters and atomic force microscopes.

\section{Contributors and Collaborators}

S.M. Khan, L. Ma, F. Tavazza, F. Biancaniello (Metallurgy Division, NIST); H. Burdette, B. Hockey, R. Wagner (Ceramics Division, NIST) 


\section{Modulus Mapping at the Nanoscale}

We are developing metrology for rapid, quantitative assessment of elastic properties with nanoscale spatial resolution. Atomic force acoustic microscopy (AFAM) methods enable modulus measurements either at a single point or as a map of local property variations.

The information obtained furthers our understanding of nanopatterned surfaces, thin films, and nanoscale structures.

\section{Donna C. Hurley}

$\mathrm{A}^{\mathrm{s}}$ s critical dimensions shrink well below $1 \mu \mathrm{m}$, new tools are required to investigate materials properties on commensurate scales. Not only is higher spatial resolution needed, but it is also increasingly important to assess not just the "average" properties from a single sample position, but to visualize the spatial distribution in properties. To meet these needs, we are developing methods to measure and image elastic properties based on the atomic force microscope (AFM). Atomic force acoustic microscopy (AFAM) involves the vibrational modes of an AFM cantilever when its tip is in contact with a sample. With AFAM, the indentation modulus $M$ of the sample can be determined. The small tip radius $(\sim 5-50 \mathrm{~nm})$ enables nanoscale spatial resolution.

In FY05, we combined single-point AFAM methods with scanning to achieve quantitative nanomechanical mapping. We developed a frequency-tracking circuit to pinpoint the contact-resonance frequencies at each image pixel. A digital signal processor architecture enables rapid data acquisition ( 20 min. per 256 x 256 image). Figure 1 shows an example of a modulus map calculated from resonant-frequency images. The calculations use a Hertzian model for the tip-sample contact. The values of $M$ agree with both single-point AFAM measurements on the same sample and literature values for the constituent materials in bulk form. This mapping capability greatly expands our ability to evaluate the properties of multicomponent nanostructures.

Accurate AFAM measurements depend on a detailed knowledge of the contact mechanics between the tip and the sample. In FY05, we continued tandem AFAM and high-resolution scanning electron microscope (SEM) experiments to better understand the contact mechanics. We found that the actual behavior varies from tip to tip and is best described by a mixture of Hertzian and flat-punch models. We are now working to integrate these results into our measurement and analysis procedures in order to improve accuracy and precision.

Another aspect of measurement accuracy involves the static forces applied by a tip. The magnitude of the

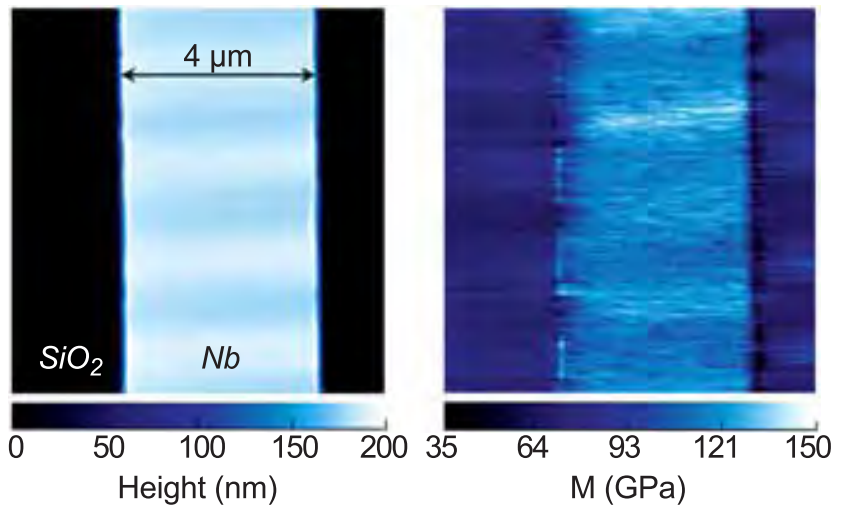

Figure 1: AFM topography (L) and AFAM modulus map (R) images of a thin-film sample containing a niobium $(\mathrm{Nb})$ stripe $\sim 200 \mathrm{~nm}$ thick on top of a silica $\left(\mathrm{SiO}_{2}\right)$ blanket film $\sim 350 \mathrm{~nm}$ thick.

force is determined by the stiffness of the cantilever, but this quantity is often poorly known. We began work on this issue in FY05 using NIST's electronic force balance (EFB). The EFB provides SI-traceable force calibration at the nano- to micronewton scale. With the EFB, we obtained the voltage-versus-force relationship for several piezoresistive cantilevers. These cantilevers will serve as force transfer standards to calibrate the cantilevers of unknown stiffness used in our experiments.

We also continued experiments on relative humidity (RH) effects using self-assembled monolayers (SAMs) of n-octyldimethylchlorosilane on silicon. The calculated contact stiffness did not depend on RH for a hydrophobic SAM, but increased strongly with RH for a hydrophilic SAM. Because the contact stiffness represents the elastic tip-sample interaction, it should not depend on humidity. The data can be explained by capillary forces that cause a viscoelastic interaction. To include capillary forces, a humidity-dependent damping term was added to the data analysis model. The contact stiffness calculated with this model was constant with RH, and the damping behavior was similar to that observed for AFM pull-off forces. Such results enhance our measurement capabilities for a range of materials and environmental conditions.

FY05 project results were described in 6 contributed and 2 invited journal articles, 5 conference presentations (3 proceedings), and 2 invited workshop presentations.

\section{Contributors and Collaborators}

M. Fasolka, R. Geiss, D. Julthongpiput, M. Kopycinska-Müller, P. Rice, D. Smith (MSEL, NIST); A. Kos (EEEL, NIST); J. Pratt (MEL, NIST); M. Prasad (Colorado School of Mines); J. Turner (University of Nebraska); W. Arnold, U. Rabe

(Fraunhofer Institute, Germany) 


\section{Reference Specimens for SPM Nanometrology}

Engineering of nanomaterials, biomaterials, and organic electronic devices hinges on techniques for imaging complex nanoscale features. In this respect, new Scanned Probe Microscopy (SPM) methods promise mapping of chemical, mechanical, and electro-optical properties, but these techniques generally offer only qualitative information. Our reference specimens, fabricated with a combinatorial design, calibrate image data from emerging SPM methods, thereby advancing these nanometrology tools.

\section{Michael J. Fasolka}

A new generation of SPM techniques intend to measure chemical, mechanical, and electro/optical properties on the nanoscale. However, contrast in new SPM images is difficult to quantify since probe fabrication can be inconsistent, and probe/sample interactions are not understood. Our research at the NIST Combinatorial Methods Center (NCMC) provides reference specimens for the quantification of next-generation SPM data. Using a gradient combinatorial design, our specimens gauge the quality of custom-made SPM probes and calibrate SPM image contrast through "traditional” surface measurements (e.g., spectroscopy and contact angle).

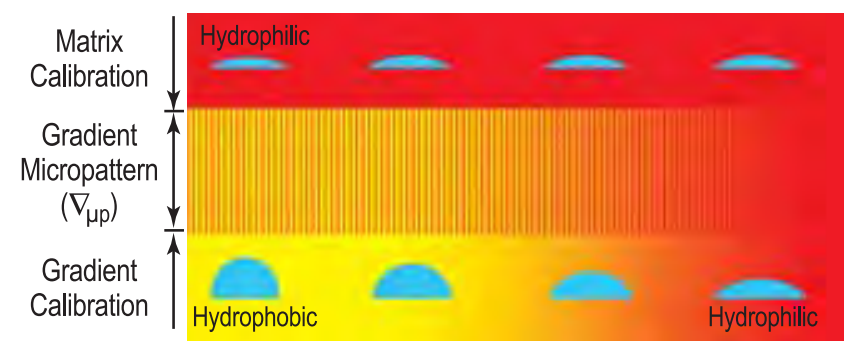

Figure 1: Schematic illustration of the $\nabla \mu$ p for calibration of chemically sensitive SPM techniques. Blue "droplets" illustrate water contact angle measurement along the calibration strips.

This year, we demonstrated the fabrication and use of a reference substrate that combines patterning of a self-assembled monolayer (SAM) with a surface energy gradient. Our gradient micropattern $(\nabla \mu \mathrm{p})$ specimens incorporate a series of micron-scale lines that continuously change in their surface energy compared to a constant matrix. Patterning is achieved via a new vapor-mediated soft lithography of a hydrophobic chlorosilane SAM on $\mathrm{SiO}_{2}$ (matrix). A subsequent graded UV-Ozone exposure gradually changes the chemistry of the patterned SAM along the specimen from hydrophobic to hydrophilic species. As shown in Figure 1, the specimen design includes two calibration fields, which reflect the changing chemistry of the SAM lines and the constant chemistry of the matrix. Accordingly, traditional measurements, e.g., water contact angle, along these fields relate the chemical contrast in the $\nabla \mu$ p to known quantities, e.g., surface energy differences.

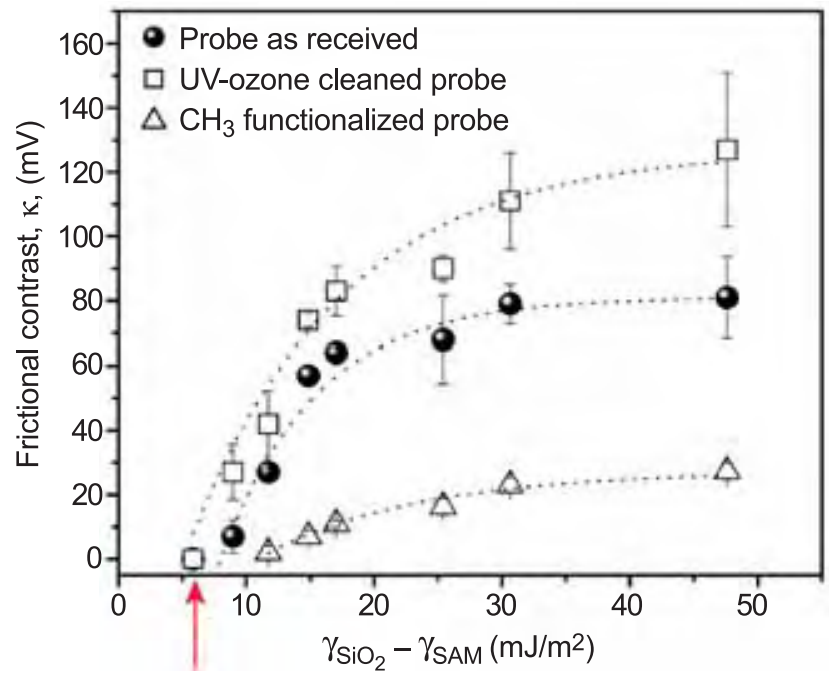

Figure 2: Demonstration of $\nabla \mu$ p specimen. SPM friction contrast $(\kappa) v$ s. surface energy $(\gamma)$ differences obtained with probes of different chemical quality. The red arrow marks the $\gamma$-difference sensitivity of a UV-ozone cleaned probe.

Figure 2 demonstrates the utility of the $\nabla \mu$ p specimen for SPM data calibration. This plot was generated from a series of SPM friction images acquired along the graded pattern. A frictional contrast parameter, $\kappa$, which reflects measured friction force differences between the lines and matrix, was extracted from each image. To create a contrast calibration curve, $\kappa$ is plotted against surface energy data derived from water contact measurements along calibration fields. As shown in Figure 2, the combinatorial $\nabla \mu$ p provides, in a single specimen, a fullspectrum relationship between SPM friction force and surface energy. As shown through the three curves, the specimen also enables direct comparison between different probe functionalization strategies. Moreover, the curves illuminate the minimum $\gamma$-difference detectable by a given probe (where $\kappa \rightarrow 0$ ), i.e., its chemical sensitivity.

Our fabrication route for the $\nabla \mu$, and its use as a reference specimen for emerging SPM techniques, is the subject of an article published in Nanoletters (2005, ASAP).

\section{Contributors and Collaborators}

K.L. Beers, D. Julthongpiput (Polymers Division, NIST); D. Hurley (Materials Reliability Division, NIST); T. Nguyen (Materials and Construction Research Division, NIST); S. Magonov (Veeco/Digital Instruments) 


\section{Nanotribology and Surface Properties}

Accurate determination of adhesive and frictional forces between surfaces and particles is critical for efficient and effective design and development of nanoscale devices and manufacturing processes. Working with diverse industrial partners (instrument, device, and magnetic storage industries), we are addressing this critical need by developing metrology tools and methods for nanomechanical property measurements.

\section{Stephen M. Hsu and Richard S. Gates}

$\mathrm{O}$ ne of the major conclusions from the National Nanotechnology Initiative workshop on instrumentation and metrology (held at NIST in January 2004) was need for improved tools, methods, and calibration procedures for nanoscale measurements. Several advances in measuring friction and adhesion and controlling surface lubrication and texturing were achieved this year.

\section{Advances in Instrumentation}

Existing atomic force microscopy (AFM) and multiscale friction testing instruments were upgraded to improve measurement accuracy and extend applicability of the methods. The AFM was extensively modified to increase the signal-to-noise ratio. A joint effort with Hysitron resulted in a new 3-D force sensor to conduct friction and scratch tests with much higher accuracy. Sample stage modulation is being implemented across several platforms to increase sensitivity and expand measurement capability. In-house cantilever and tip fabrication capability and collaborations with numerous specialty tip fabricators were established.
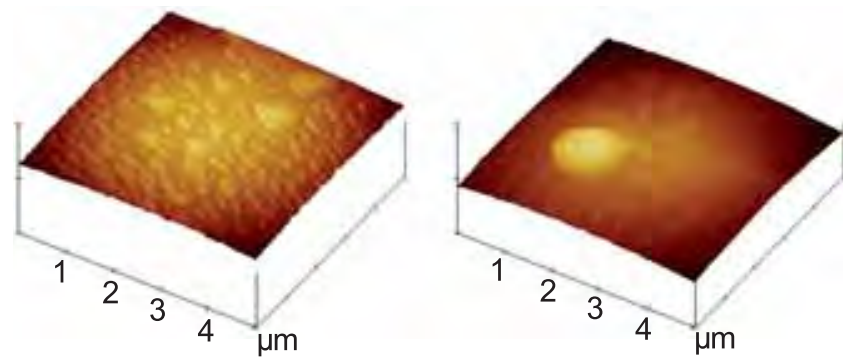

Figure 1: Surface features on colloidal probes.

In adhesion and friction measurements, surface forces are critical parameters that depend upon the real area of contact. Figure 1 illustrates typical colloidal probes showing random surface features. A computational procedure was developed to estimate the bearing area for this type of probe enabling better determination of contact areas.

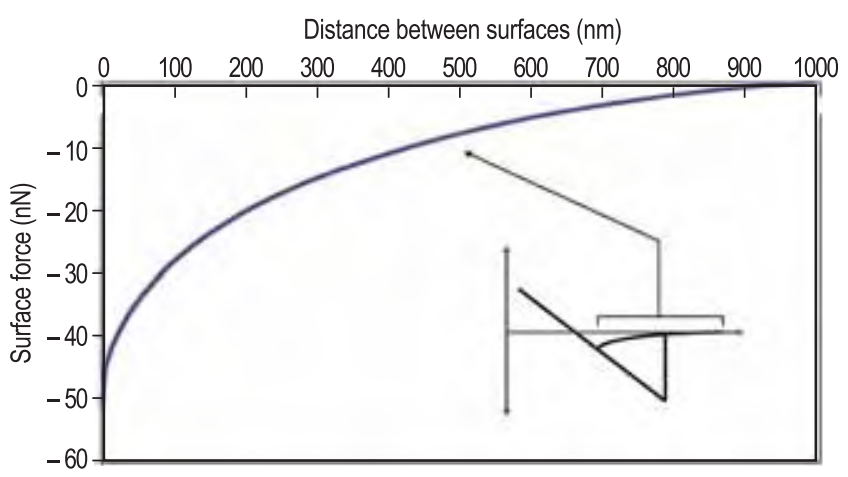

Figure 2: Surface force measurement down to $1 \mathrm{~nm}$. The inset shows a schematic of a typical force curve from the AFM.

The first nanoscale probe using an ultra-thin sheet of mica glued on a colloidal probe was successfully developed to measure surface forces on extremely small areas (Figure 2). To avoid snap-on of the probe tip during approach, the cantilever stiffness and signal-to-noise ratio were increased.

\section{Surface Control}

Advances were made in the organization of mixed molecules on surfaces for hydrophobicity, anti-adhesion, and friction control properties. Ultra-durable hydrophobic films and friction control films were demonstrated last year. Collaboration with Dan Fischer at the NIST beam line at the National Synchrotron Light Source continues to be vital in characterizing these complex molecular mixtures.

Surface textures are increasingly being used to control surface energy, polarity, adhesion, and friction. In work supported by other agencies and industries, the surface properties of materials were controlled by use of specific surface features such as dimples, triangles, and ellipses at micro- and nanoscale dimensions.

\section{Interactions}

Ongoing interactions with domestic and international partners included a cantilever force calibration study with other National Measurement Institutes and Jon Pratt (NIST, MEL), and surface texture research with seven other countries.

\section{Contributors and Collaborators}

C. Ying, S. Yang, M. Reitsma, D.-I. Kim, Y. Liang, X. Wang, J. Grobelny, D. Fischer, Y.T. Hsia (Seagate); W. Gerberich (University of Minnesota); O. Warren (Hysitron); C. Su (Veeco); D. Mendel (NPL);

E. Santner (BAM) 


\section{Chemistry and Structure of Nanomaterials}

Successful nanoscale materials fabrication is empowered by a detailed knowledge of the chemistry and structure of surface bound molecules; e.g., the optimization of SAMs, molecular templates, MEMs lubricants, and functionallized nanotubes. We develop, demonstrate, and advance cutting-edge synchrotron metrologies to bring nanoscale materials phenomena to practical applications.

\section{Daniel A. Fischer, Vincent A. Hackley, and Andrew J. Allen}

Tn potential MEMs lubricants, we have found that 1 the degree of surface ordering in self-assembled monolayers (SAMs) governs the friction properties of the film. n-Alkyltricholorosilanes films with different chain lengths ( $\mathrm{C}$ films where $\mathrm{n}=5-30$ ) were characterized by near-edge $\mathrm{x}$-ray absorption fine structure (NEXAFS), Fourier transform infrared spectroscopy (FTIR), and atomic force microscopy (AFM). The chain lengths having 12, 16 and 18 carbon atoms were found to be highly oriented with a preferential molecular orientation of the polymeric C-C chains perpendicular to the surface. C5 and C30 SAMs did not exhibit preferential orientation of the alkyl chain and C10 showed partial ordering. Complementary FTIR tudies were done to estimate order qualitatively by peak positions of asymmetric $\mathrm{CH} 2$ and the symmetric $\mathrm{CH} 2$ stretches. The molecular order information from FTIR followed similar trends as determined by NEXAFS. The frictional properties of the organic monolayers were determined through the simultaneous measurement of normal (load) and lateral (friction) interfacial forces with AFM. Friction measurements on different chain lengths follow inverse trends with surface order from NEXAFS as shown in Figure 1.

\section{Dichroic Ration Trend in Tricholorosilane SAM}

\section{Friction Trend in Tricholorosilane SAM}

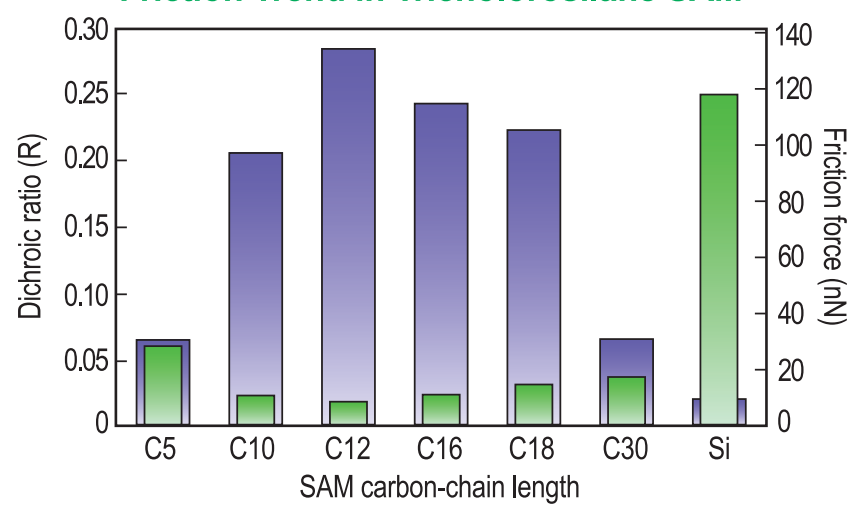

Figure 1: SAM order and friction versus chain length.
The flow-cell developed by NIST for in situ ultra-small-angle x-ray scattering (USAXS) studies of solution-mediated nanoscale materials has been applied to the technologically important case of a homogeneously precipitating solution of nanosize ceria $\left(\mathrm{n}-\mathrm{CeO}_{2}\right) \cdot \mathrm{n}-\mathrm{CeO}_{2}$ has multiple applications in catalysis, as a solid oxide fuel cell electrolyte material, and in a number of other areas.

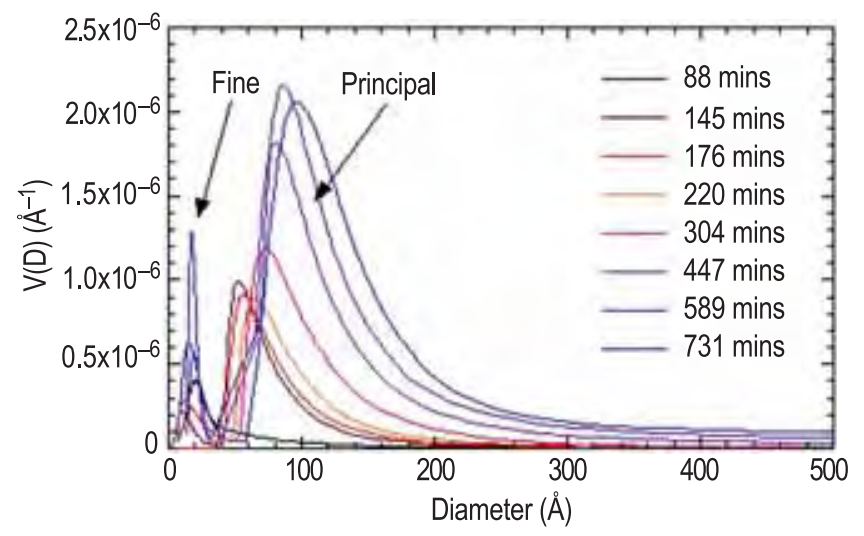

Figure 2: Homogeneous precipitation of $n-\mathrm{CeO}_{2}$.

Modeling of in situ USAXS data taken during reaction at $25{ }^{\circ} \mathrm{C}$ indicates a co-precipitation of solid principal particles (see Figure 2) and a population of fine particles with a core-shell morphology. The principal population grows in size and volume fraction, $\mathrm{V}$, but the fine secondary features grow only in volume fraction. It has been postulated that the fine features constitute a step in the formation of the principal particle population, a theory currently being tested with additional experiments in conjunction with Columbia University collaborators.

A strong temperature affect is seen: at $35{ }^{\circ} \mathrm{C}$, the volume fraction of principal particles increases with a growth rate roughly twice that at $25^{\circ} \mathrm{C}$. The activation energy for the $\mathrm{n}-\mathrm{CeO}_{2}$ precipitation was estimated from USAXS data to be about $46 \mathrm{~kJ} \mathrm{~mol}^{-1}$. Additional studies with the flow-cell focus on dispersion and flow-induced alignment of carbon nanotubes in various solvent/dispersant systems.

\section{Contributors and Collaborators}

S. Sambasivan, S. Hsieh, S. Hsu (Ceramics Division, NIST); P.R. Jemian (University of Illinois); J. Ilavsky (APS XOR); S.-W. Chan (University of Columbia); J. Randall, M. Banesh (Zyvex Inc.); H. Wang (Michigan Tech.) 


\section{Nanotube Processing and Characterization}

Single-wall carbon nanotubes (SWNTs) exhibit remarkable physical properties, and there is considerable interest in using them as nanoscale building blocks for a new generation of applications. Despite this promise, fundamental issues related to the dispersion, fractionation, orientation, and manipulation of individual single-walled carbon nanotubes remain unresolved, and efficient bulk processing schemes do not exist. We are working at the scientific front of this rapidly emerging field to establish research protocols that will help ensure that this new technology progresses as quickly and efficiently as possible, but with uniformly high standards.

\section{Barry J. Bauer, Kalman Migler, and Erik K. Hobbie}

$\mathrm{U}$ pon their discovery in 1991, carbon nanotubes were recognized as ideal materials for nanotechnology applications. Properties of carbon nanotubes differ vastly depending on their diameter and chirality, and interest in these materials stems from their extraordinary combination of properties: superior thermal conductivity, electrical conductivity, and mechanical strength. Nanotubes are thus attracting great attention for emerging technologies such as bio-chemical sensors, next generation displays, and nano-electronics. Regardless of the ultimate applications, nanotubes clearly represent the most important new class of materials in the past 15 years.

However, application development is plagued by inconsistent sample quality, compounded by a lack of consensus on material characterization methods and by

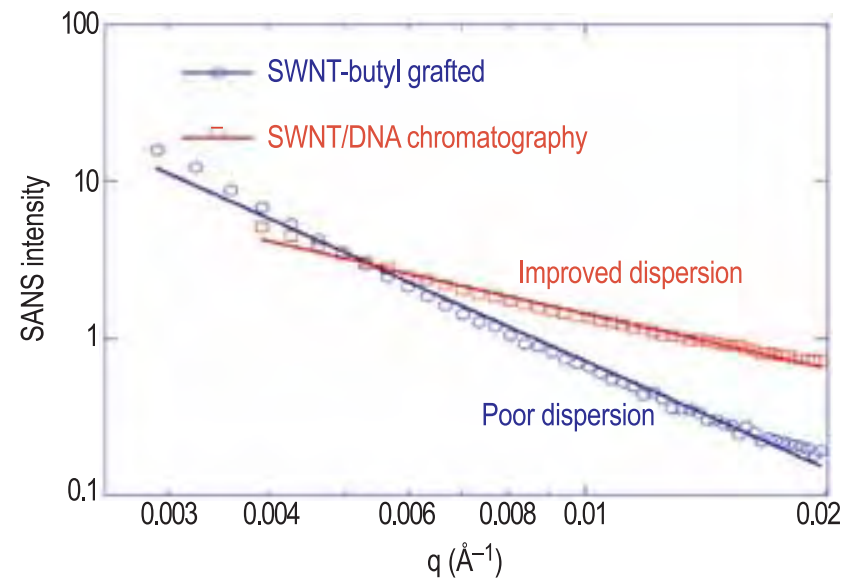

Figure 1: Measured SANS profiles obtained for two different SWNT dispersion chemistries, showing how DNA wrapping provides superior dispersion to other methods, such as chemical functionalization. poor measurement reproducibility. The quality problems plaguing the nanotube community were described in a recent news article in Nature which stated, "the situation will not improve until an external body introduces standards that suppliers can follow." Few people were surprised by the conclusion of the recent workshop: NIST must take the lead in a quantitative nanotube metrology that will allow suppliers and customers to develop standards for the developing industry.

The Nanotube Processing and Characterization Project within the Polymers Division is actively engaged in this effort. As a starting point, we are currently using small-angle neutron scattering (SANS) to quantify the degree of SWNT dispersion using a variety of dispersion chemistries (Figure 1) and, in doing so, have identified DNA wrapping as desirable for the purpose of fractionating SWNTs by length, diameter, chirality, and band structure.

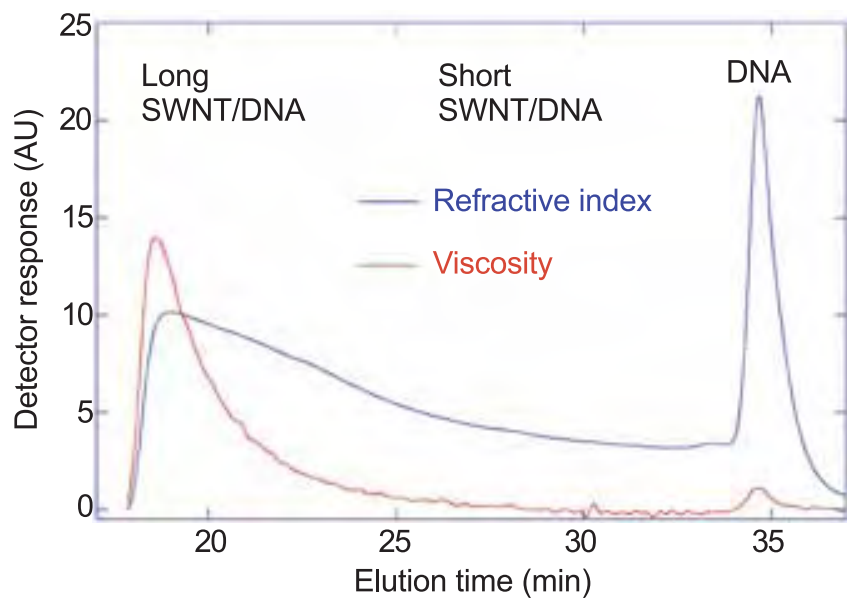

Figure 2: Refractive index and viscosity as a function of elution time in a size-exclusion chromatograph from DNA wrapped SWNTs, showing clear separation by length.

Taking this one step further, we have begun using size-exclusion and ion-exchange chromatography to sort SWNTs by length and chirality (Figure 2). Following the protocol pioneered by DuPont researchers, we are producing ultra clean SWNT fractions that will be characterized with a broad suite of NIST metrologies. These results will in turn be used to establish universal scientific standards for SWNT purity and dispersion.

\section{Contributors and Collaborators}

W. Blair (Polymers Division, NIST); A. Hight Walker (Optical Technology Division, NIST); T. Yildirim (NIST Center for Neutron Research); M. Pasquali (Rice University); M. Zheng (DuPont) 


\section{Carbon Nanotube Applications: The Role of Nanotube Alignment}

Carbon nanotubes are currently being produced by over 50 companies worldwide, with scale-up progressing at a rapid rate. Of particular importance for many potential applications is nanotube alignment, which can affect thermal, mechanical, and electrical properties. We are developing methods to deposit and grow aligned nanotubes on a variety of measurement platforms, as well as to measure the degree of alignment achieved.

\section{Stephanie A. Hooker}

$\mathrm{C}$ arbon nanotubes are truly revolutionary materials, exhibiting properties vastly different than any other bulk form of carbon. Their unique combination properties make them valuable for electronics, advanced structures, biotechnology, and thermal management. However, reliable property measurement continues to prove difficult due to supplier variability, difficulties manipulating materials at this small scale, and physical and chemical interactions (e.g., with substrates, contacts, other nanotubes, and the surrounding environment). Alignment of the individual nanotubes also plays a significant role in dictating ultimate performance.

One potential application for which alignment is particularly critical is absorptive coatings for infrared detectors. Many such detectors are based on pyroelectric crystals that become electrically polarized with temperature changes. To enhance response, coatings are applied to improve heat transfer. Carbon nanotubes are an excellent choice for such coatings due to their high thermal transport, which can be tailored by varying diameter and orientation (i.e., alignment). Alignment in such coatings is often achieved using chemical vapor deposition (CVD). Unfortunately, however, pyroelectric crystals present new challenges for CVD growth, as high temperatures, reducing atmospheres, and metal catalysts are required. When these are
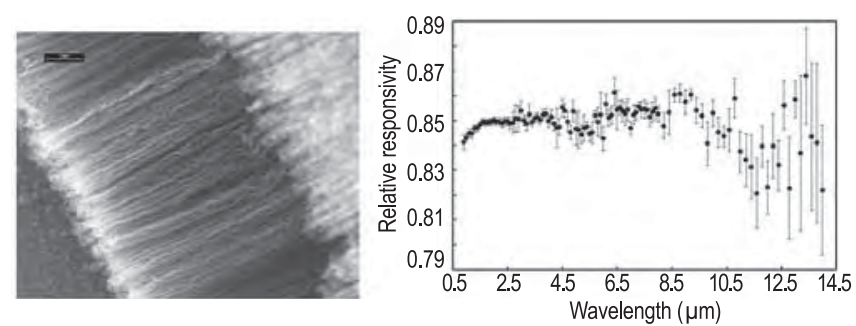

Figure 1: SEM image of vertically aligned nanotubes grown on $\mathrm{LiNbO}_{3}$ at $725^{\circ} \mathrm{C}$. Critical to the process is a thin silicon nitride $\left(\mathrm{Si}_{3} \mathrm{~N}_{4}\right)$ barrier layer, preventing catalyst diffusion into the crystal. The graph shows the associated spectral response of the crystal after application of the coating. combined, undesirable (and irreversible) changes in the pyroelectric crystal are observed. Recently, however, we demonstrated a new combination of catalyst, barrier layer, growth temperature, and processing gas mixture that permits direct synthesis of vertically aligned nanotube arrays on lithium niobate $\left(\mathrm{LiNbO}_{3}\right)$ and lithium tantalate $\left(\mathrm{LiTaO}_{3}\right)$ pyroelectric crystals (Figure 1).
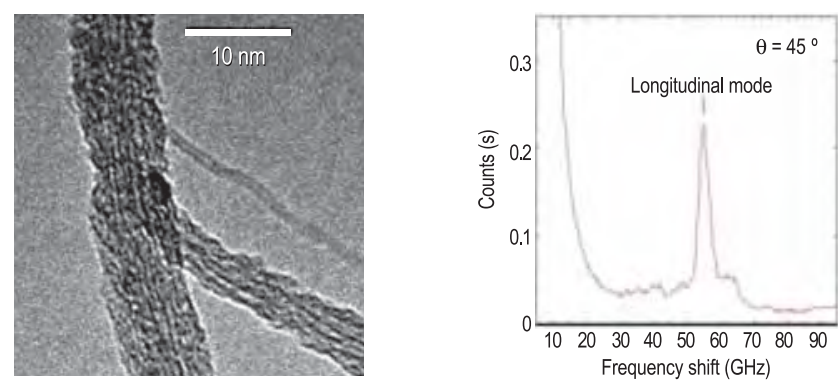

Figure 2: TEM image of a single-wall carbon nanotube rope (courtesy of Rice University). The high degree of alignment in the rope was observed in the BLS spectrum to the right.

Nanotube alignment is also important for many other applications, including those in electronics and advanced structures. One measurement technique with promising sensitivity to tube alignment is Brillouin Light Scattering (BLS). In FY05, we used BLS to evaluate a highly-aligned single-wall nanotube rope. The resulting spectra showed a distinct peak associated with the rope, much different than the broader pattern previously reported for a more randomly aligned nanotube network. BLS is also being explored in conjunction with nanotube separation techniques to provide an indication of size exclusion.

In FY05, we also helped organize a 2nd joint NASA-NIST workshop on Measurement Issues in Single-Wall Carbon Nanotubes. This meeting, held January 26-28, 2005, addressed challenges in measuring purity and dispersion quality. Over 75 technical and business leaders participated, debating a wide portfolio of characterization tools including Raman spectroscopy, near-IR spectroscopy, thermogravimetric analysis, and electron microscopy. These techniques are currently being drafted into a Recommended Practice Guide, which will then be adapted by IEEE into a Purity Standard for Carbon Nanotubes.

\section{Contributors and Collaborators}

W. Johnson, S. Kim, T. Oreskovic, P. Rice, V. Tewary, N. Varaksa (Materials Reliability Division, NIST); K. Migler (Polymers Division, NIST); S. Freiman (MSEL); S. Arepalli, L. Yowell (NASA-JSC); R. Mahajan (University of Colorado); R. Hauge (Rice University) 


\section{Combinatorial Adhesion and Mechanical Properties}

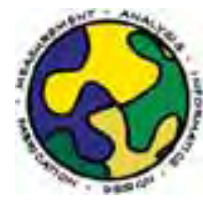

Traditional methods for evaluating the engineering properties of polymers are time-consuming and inherently single specimen tests. Current market drivers increasingly demand rapid measurement platforms in order to keep pace with competition in the global marketplace. In this project, we are delivering innovative combinatorial and highthroughput (C\&HT) tools for the physical testing of materials, built around measurement platforms in the NIST Combinatorial Methods Center (NCMC).

\section{Christopher M. Stafford}

$\mathrm{O}$ ur current C\&HT efforts in this project are concentrated in two main areas: buckling mechanics for thin film mechanical measurements and adhesion testing platforms for probing interfacial adhesion and fracture. Here, we highlight: (1) the inversion of our buckling-based metrology to study the mechanical response of soft polymer gels, (2) application of finite element analysis to study buckling in multilayer geometries, and (3) the implementation of our combinatorial edge delamination test to study the interfacial adhesion strength of epoxy films.

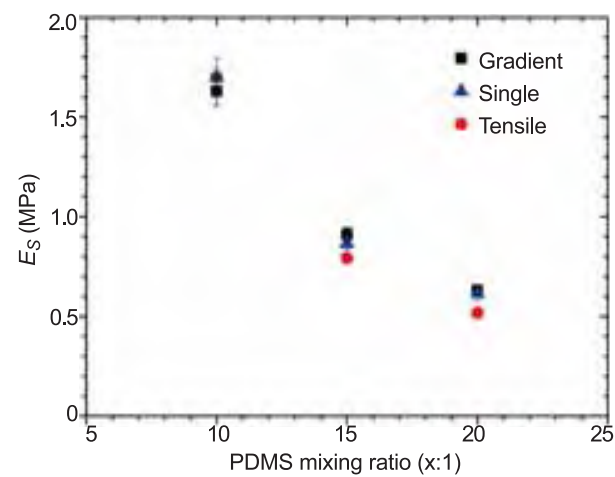

Figure 1: Elastic modulus of model PDMS gels measured via buckling (- - gradient modulus specimen, $\mathbf{\Delta}$ - single specimens) and via tensile test $(\bullet)$.

This year, we applied our buckling-based metrology ${ }^{[1]}$ to measure the elastic modulus of soft-polymer gels. Elastic modulus is an important design criterion in soft polymer gels for biomedical applications since it impacts critical properties such as adhesion, swelling, and cell proliferation and growth. Leveraging our C\&HT buckling-based metrology, we can rapidly assess the elastic modulus of polymer gels by inverting the experimental design: the buckling of a sensor film of known modulus and thickness reports the elastic modulus of the substrate, $E_{S}$. Figure 1 illustrates the accuracy of our approach as compared to traditional tensile tests on the same material. One advantage of our buckling-based technique is that a "modulus map" can be constructed by measuring the buckling wavelength as a function of spatial position.

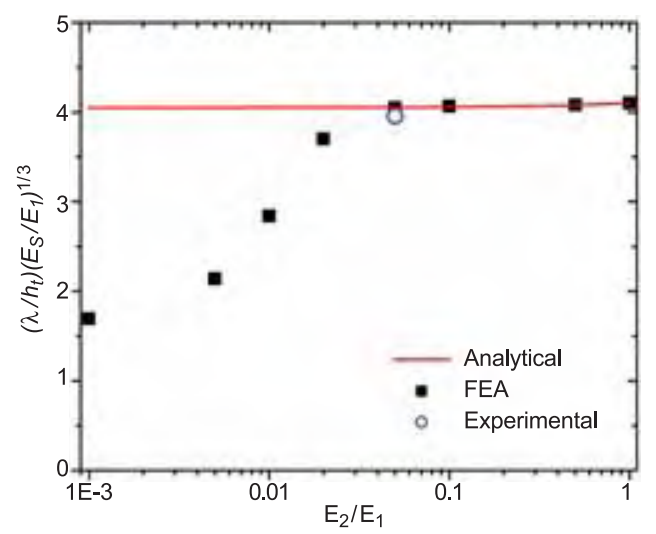

Figure 2: Normalized wavelength versus modulus ratio of a tri-layer thin film. $E_{2}$ and $E_{1}$ are the moduli of the soft and stiff layer, respectively. The solid line is the analytical solution.

In addition to our experimental efforts, we are also utilizing finite element analysis (FEA) to help guide experimental design in our buckling-based metrology by verifying the validity of available analytical solutions when applied to more complex specimen geometries. For example, we examine a composite film consisting of a soft layer confined between two stiff layers. In Figure 2, FEA reveals a critical modulus ratio below which shear deformation becomes significant, thus the standard analytical solution can no longer be applied to measurements in this regime.

As part of the NCMC, we have launched a Focus Project aimed at developing a C\&HT measurement platform for testing interfacial adhesion and fracture in thermally cured epoxy materials. This method is based on the modified edge-liftoff test. ${ }^{[2]}$ In this Focus Project, we are building capabilities to evaluate the governing parameters for interfacial delamination and reliability by fabricating suitable gradient libraries in composition, thickness, temperature, and applied stress. Industrial sponsors for this Focus Project are ICI National Starch and Intel Corporation.

1. C.M. Stafford, et al. Nature Materials 3, 545 (2004).

2. M.Y.M. Chiang, et al. Thin Solid Films 476, 379 (2005).

\section{Contributors and Collaborators}

M.Y.M. Chiang, S. Guo, J.H. Kim, E.A.Wilder, W. Zhang (Polymers Division, NIST); Daisuke Kawaguchi (Nagoya University); Gareth Royston (University of Sheffield) 


\section{Soft Nanomanufacturing}

Nanomanufacturing is widely noted as a central challenge of nanotechnology. In the realm of soft materials and suspended particles, it is necessary to design particle interactions, manipulate self-assembly processes, and measure what is produced. Guided by theoretical simulations, we are therefore developing high-throughput microfluidic methods for particle characterization, processing, assembly, and on-chip quality control.

\section{Steven D. Hudson}

$\mathrm{T}_{\mathrm{d}}^{\mathrm{h}}$ he intricacy of biological systems inspires the design of artificial systems that also function through dynamic self-assembly and in-situ monitoring and self-correction.

Our industrial partners identified measurement of interfacial tension as a first hurdle for high-throughput microfluidic fluids analysis. Particle processing and assembly methods represent the next hurdle. In this project, high-throughput tools are developed for these purposes, and theoretical simulations identify particle arrangements whose dynamic assembly and disassembly is promising for sensor applications.

High-throughput measurement of drop shape by image analysis represents the cornerstone of an instrument, developed in collaboration with industrial sponsors, that determines interfacial tension between fluids. The measurement principle is simple and robust - drops are stretched by known viscous forces as they traverse a constriction in the channel. The computer controlled system tracks drop position and deformation more than one hundred times a second.

However, systems that count, isolate, and direct the assembly of particles must operate more efficiently to enable internal feedback mechanisms. Therefore, for
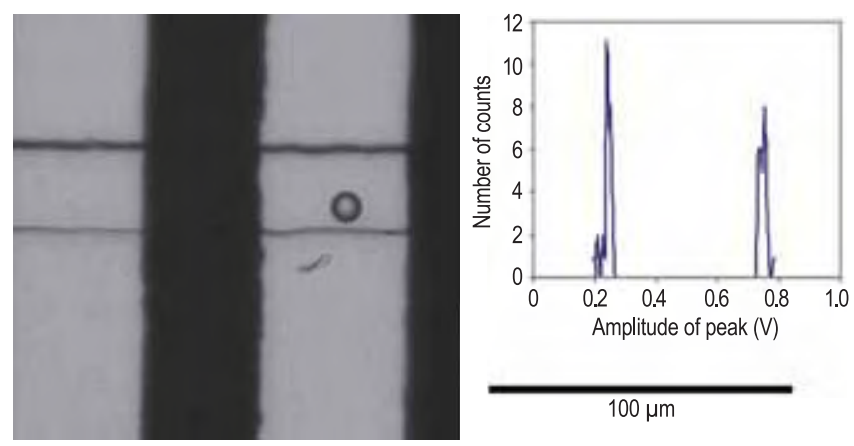

Figure 1: Inline particle characterization and counting. In the image, a polystyrene particle is seen passing electrodes (dark). At right is shown a bimodal size distribution of liquid drops produced at a T-junction upstream. assembly applications, image analysis was replaced by embedded electronic sensors that detect the presence of a particle and its size (Figure 1). This electronic signal activates a valve to isolate a set of particles. Electronic detection is further advantageous because it is readily scalable to smaller particles. In comparison to previous systems, the valves we have developed are also advantageous, since they are not limited to shallow channel profiles.

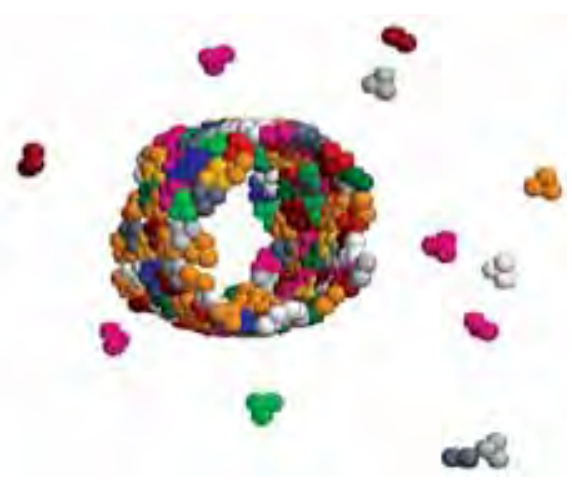

Figure 2: Tubular structures (right) arise from the simulated organization of triangular arrangements of dipole particles, shown schematically at left.

High-throughput sensing and processing methods require precision flow design and control, such as we demonstrated and reported previously by a microfluidic analog of the four-roll mill. Advancing beyond, we developed a framework for generating chaotic flow in microchannels (described in a separate highlight).

Theoretical simulations probe the organization of geometrically and electrostatically asymmetric target particle arrangements (Figure 2). These demonstrate the relationship between particle symmetry and organized structure. Depending on this symmetry, the assemblies exhibit filaments, sheets, tubes and icosahedra. Whereas ordinary phase separation is driven by attractive and repulsive interactions, self-assembly of more complex and finite-sized structures requires directional interactions.

Of consequence for sensor applications, organization kinetics were also investigated. In particular, nucleating agents were found to control the kinetics of assembly and, in polymorphic systems, to specify unique structure.

\section{Contributors and Collaborators}

F. Phelan, Jr., J. Douglas, K. Migler, H. Hu, P. Stone, J. Taboas, K. VanWorkum (Polymers Division, NIST); Y. Dar, S. Gibbon (ICI/National Starch); M. McDonald (Procter \& Gamble); D. Discher, V. Percec (University of Pennsylvania); R. Tuan (NIH) 


\section{Defects in Polymer Nanostructures}

Nanostructured materials create new and unique functionality through the accurate placement, precise shaping, and chemical modification of nanometer scale patterns. Such materials are to be the basis of a wide range of emerging nanotechnologies that span optics, data storage, and biomembranes. In each of these applications, defects in pattern placement, shape, and chemical composition can compromise device functionality. The rapid development of these technologies is currently offset by a lack of quantitative characterizations of critical defects. We have initiated this project to develop metrologies for characterizing critical defects, such as loss of long-range order, in nanostructured materials.

\section{Ronald L. Jones and Alamgir Karim}

$\mathrm{T}$ he optical, magnetic, and electronic properties of a film or surface are dramatically changed by the inclusion and placement of nanometer-scale patterns. The capability to adjust material properties in this manner is central to the development of sub-wavelength optics, high selectivity biomembranes, nanoparticle synthesis, and ultrahigh capacity data storage. In each of these applications, variations in pattern shape and placement can drastically alter functionality and device viability.

Fabrication of nanostructured surfaces is performed through a wide range of patterning platforms. While photolithographic techniques are traditional routes toward precise patterning, the high cost and complexity of patterning at nanometer length scales has spawned a variety of alternative techniques such as nanoimprint lithography (NIL), self-assembly, and templated self-assembly. Each fabrication technique strives against a common set of critical defects such as variation in pattern placement, chemical uniformity across the pattern cross section, and precision in pattern shape.

To address the needs of this emerging technological area, we have initiated a new program to develop metrologies for long-range order, a critical parameter in optical and data storage applications. Currently, long-range order is quantified from Fourier transforms of real-space microscopy images. However, the disparity in the pattern length scale $(\sim 10 \mathrm{~nm})$ and the length scale of ordering $(\sim 100 \mu \mathrm{m})$ challenges the measurement range of existing techniques based on scanning electron and scanning probe microscopies. Visible light probes are often complicated by complex interactions with nanometer scale features.
Using small angle x-ray scattering (SAXS), we are developing quantitative descriptions of long-range order, grain size, and pattern shape in hexagonally arrayed cylinders produced on silicon substrates using both nanoimprint lithography (NIL) and self-assembled block copolymers (BCP).

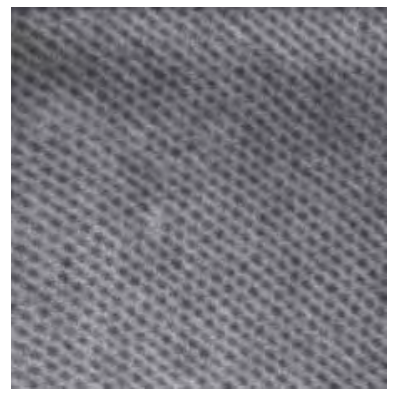

Figure 1: Scanning electron microscopy image showing a regular array of hexagonally packed columns formed in silicon oxide.
For each method of pattern formation, long-range order results in a characteristic diffraction pattern. However, the occurrence of a hexagonal diffraction pattern from the NIL pattern indicates a single crystal spanning the entire $150 \times 150 \mu \mathrm{m}$ beam spot, while the BCP film consists of multiple, randomly oriented crystals. In both cases, systematic errors in the placement of the patterns on the lattice create a characteristic decay in the intensity as a function of the distance from the beam center.

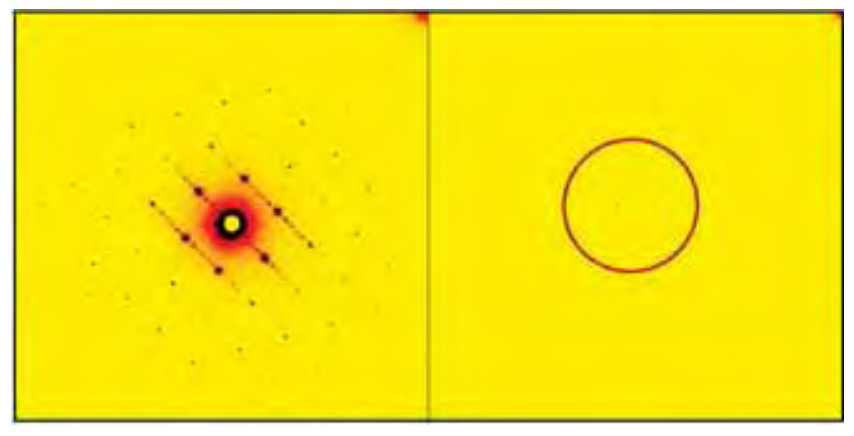

Figure 2: SAXS data from arrays of hexagonally packed columns formed by NIL (left) and self assembly (right).

In addition to developing metrologies for long-range order, we continue to develop a suite of metrologies that address critical needs in a wide range of nanostructured materials applications. These include nanostructured surfaces for adhesion and wettability, as well as nanostructured materials designed for unique electro-optical and magnetic properties.

\section{Contributors and Collaborators}

J.F. Douglas (Polymers Division, NIST); S. Satija (NIST Center for Neutron Research); R. Briber (University of Maryland); H.C. Kim (IBM) 


\section{Critical Dimension Small Angle X-Ray Scattering}

The feature size in microelectronic circuitry is ever decreasing and now approaches the scale of nanometers. This creates a need for new metrologies capable of non-destructive measurements of small features with sub-nm precision. NIST has led the effort in developing small angle $x$-ray scattering to address this need. This $x$-ray based metrology has been included in the ITRS roadmap as a potential metrological solution for future generation microelectronics fabrication. Other applications of this technique in areas such as nano-rheology and nanofabrication are being explored.

\section{Wen-li Wu and Ronald L. Jones}

$\mathrm{T}_{\mathrm{d}}^{\mathrm{h}}$ he demand for increasing computer speed and decreasing power consumption continues to shrink the dimensions of individual circuitry components toward the scale of nanometers. When the smallest, or "critical", dimensions are $<40 \mathrm{~nm}$, the acceptable tolerance will be $<1 \mathrm{~nm}$. This creates significant challenges for measurements based on electron microscopy and light scatterometry. Device viability also requires the measurement be non-destructive. In addition, the continuing development of new materials for extreme ultraviolet photoresists, nanoporous low-k dielectrics, and metallic interconnects all require high-precision dimensional measurements for process development and optimization.

To address industrial needs, we are developing a high-precision x-ray based metrology termed Critical Dimension Small Angle X-ray Scattering (CD-SAXS). This technique is capable of non-destructive measurements of test patterns routinely used by microelectronic industries to monitor their fabrication process. A collimated monochromatic x-ray beam of sub- $\AA$ wavelength is used to measure the pattern dimensions on a substrate in transmission mode. CD-SAXS has previously demonstrated a capability for sub-nm precision for periodicity and line width measurements.

This year, we have extended the capabilities to provide more detailed quantifications of the pattern cross section. This includes both basic dimensions, such as pattern height and sidewall angle, as well as the depth profile of the sidewall damage of nano-patterned low-k dielectrics. The capability to provide basic dimensions is complementary to existing analyses provided by SEM, however CD-SAXS offers significant advantages in its non-destructive capability. In contrast to visible light scatterometry, detailed information on refractive indices and composition of the pattern are not required for data reduction. These capabilities and the ability to measure patterns approaching dimensions of $10 \mathrm{~nm}$ have led to the inclusion of CD-SAXS on the International Technology Roadmap for Semiconductors (ITRS) as a potential metrology for the $45 \mathrm{~nm}$ technology node and beyond.

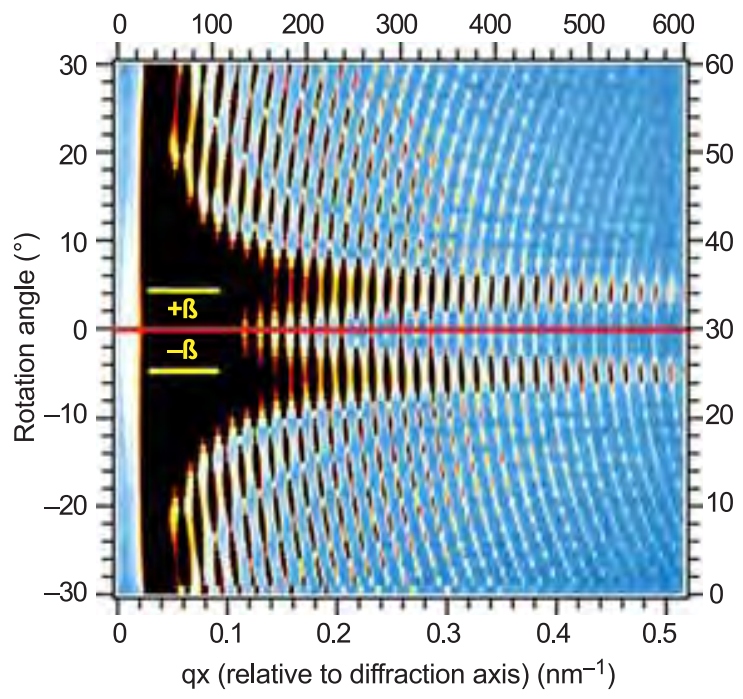

Figure 1: Diffraction patterns collected over a range of sample rotation angles. The distance between the pronounced horizontal ridges provides the sidewall angles $\beta$, while the relative intensity and placement of the other diffraction spots provide periodicity, line width, and line height. $\left[q_{x}=4 \pi \sin (\theta / 2) / \lambda\right.$, where $\theta$ is the angle relative to the diffraction axis and $\lambda$ is the wavelength of the radiation.]

So far, all CD-SAXS measurements have been carried out at the Advanced Photon Source of Argonne National Laboratory. As an important step in demonstrating the potential of technology transfer, we are constructing the world's first laboratory based CD-SAXS instrument. When completed, this instrument will serve as a prototype for lab-based tool development as well as a world-class metrology tool for nanotechnology research.

Future efforts will develop capabilities for quantifying defects and features with complex shapes such as vias or contact holes. In addition, we will continue to expand efforts in supporting other nanofabrication technologies such as those based on nanoimprint and self assembly.

\section{Contributors and Collaborators}

C. Soles, H. Lee, H. Ro, E. Lin (Polymers Division, NIST); K. Choi (Intel); D. Casa, S. Weigand, D. Keane (Argonne National Laboratory); Q. Lin (IBM) 


\section{Nanoimprint Lithography}

\begin{abstract}
Nanoimprint lithography (NIL) is emerging as a viable next generation lithography with a high throughput and a patterning resolution better than $10 \mathrm{~nm}$. However, wide-spread availability of such small nanoscale patterns introduces new metrology challenges as the ability to pattern now surpasses the capability to measure, quantify, or evaluate the material properties in these nanoscale features. We develop high-resolution metrologies to augment and advance NIL technology, with current focus on characterizing shape and the fidelity of pattern transfer.
\end{abstract}

\section{Christopher L. Soles and Ronald L. Jones}

$\mathrm{N}$ anoimprint Lithography (NIL) is a conceptually simple process whereby nanoscale patterns are written once into a master, typically Si, quartz, or some other hard material, using a high resolution but slow patterning technology such as e-beam lithography. This master can be rapidly and repeatedly replicated by stamping it into a softer resist film. This imprint replication technique is a cost-effective way to combine the high-resolution patterning of e-beam lithography with the high throughput of a stamping process.
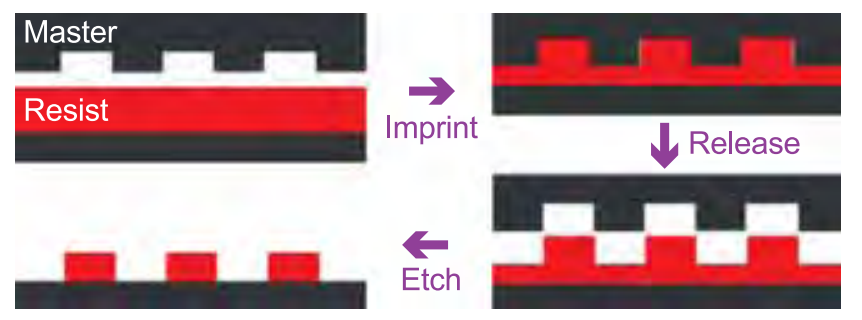

NIL holds great promise in semiconductor fabrication. The high resolution and low cost of ownership make NIL attractive in comparison to expensive next-generation optical lithography tools. However, over the past few years, the interest in NIL has dramatically expanded beyond the realm of traditional CMOS applications. NIL-based solutions are being implemented for optical communications, memory, displays, and biotechnology. Because of these emerging niche applications, NIL is quickly becoming a widely used and versatile nanofabrication tool.

Our objective is to develop metrologies that are crucial to advancing NIL as an industrially viable patterning technology. Initial efforts have focused on developing and applying very accurate pattern-shape measurements. Critical dimension small angle x-ray scattering (CD-SAXS) is a transmission x-ray scattering technique that can quantify the pattern pitch and cross-section (height, width, side-wall angle) with $\mathrm{nm}$ resolution. Likewise, specular $\mathrm{x}$-ray reflectivity (SXR) was introduced to quantify the pattern cross-section and the residual layer thickness with nm resolution. In turn, these accurate shape metrologies enable quantitative studies of imprint resolution and the stability of nanoscale imprinted patterns.

Using CD-SAXS, we demonstrated the fidelity of pattern transfer concept. The pattern cross-sections in the master and the imprint were independently characterized and then compared, to quantify how well the resist material fills and replicates the features of the master. Varying the imprint temperature, pressure, time, and the molecular mass of the imprint material impacts the fidelity of pattern transfer process. We also tracked the in-situ evolution of the cross-section while the patterns were annealed close to their glass transition. Rather than a viscous decay, the patterns decreased in height much faster than they broadened in width, owing to the residual stresses in the structures induced by the imprinting procedure. These residual stresses appear to increase with molecular mass, leading to faster rates of pattern decay in higher molecular mass resists.

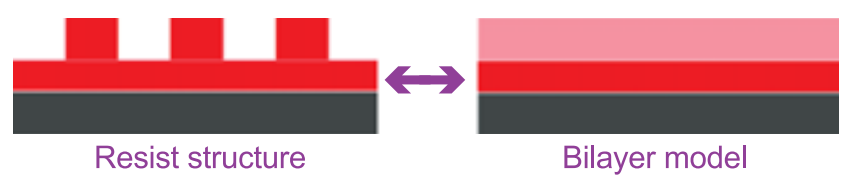

SXR was used to quantify the residual layer thickness, pattern height, and the relative line shape cross-section. The residual layer is the thin layer of resist that the master is unable to fully displace as it is pressed into the resist film. Precise knowledge of the residual thickness is critical for subsequent etching processes. The key to this measurement is that the $\mathrm{X}$-rays average density over length scales larger than the sub- $\mu$ m dimensions of the patterns. This leads to the bilayer equivalency model shown above where the patterns can be modeled as a uniform layer of reduced density to extract pattern height and residual layer thickness with nm precision. Like CD-SAXS, SXR quantitatively compares the imprint and the mold to evaluate the fidelity of pattern transfer.

\section{Contributors and Collaborators}

H.W. Ro, H.-J. Lee, A. Karim, E.K. Lin, J.F. Douglas (Polymers Division, NIST); W. Wu (MSEL Office, NIST); S.W. Pang (University of Michigan); D.R. Hines (University of Maryland); C.G. Willson (University of Texas-Austin); L. Koecher (Nanonex); D. Resnick (Molecular Imprints) 


\section{Nanomagnetodynamics}

In order to pursue the rapid development path set out for hard drives and magnetic memory, industry needs the ability to measure and control magnetization on nanometer length scales and nanosecond time scales. This project focuses on the metrology of spin dynamics and damping in magnetic thin films, especially on the properties of lithographically prepared edges of magnetic films.

\section{Robert D. McMichael}

$\mathrm{W}$ e develop measurement techniques to determine the static and dynamic properties of both continuous and patterned thin films of technologically important ferromagnetic metals and their interfaces with normal metals. The primary results this year include measurements of the affects of interfaces and interface roughness on magnetization dynamics and proof-of-concept experiments for measurements of lithographically prepared magnetic edges. These results have been communicated to the magnetic data storage industry through conference presentations, journal articles, and site visits.

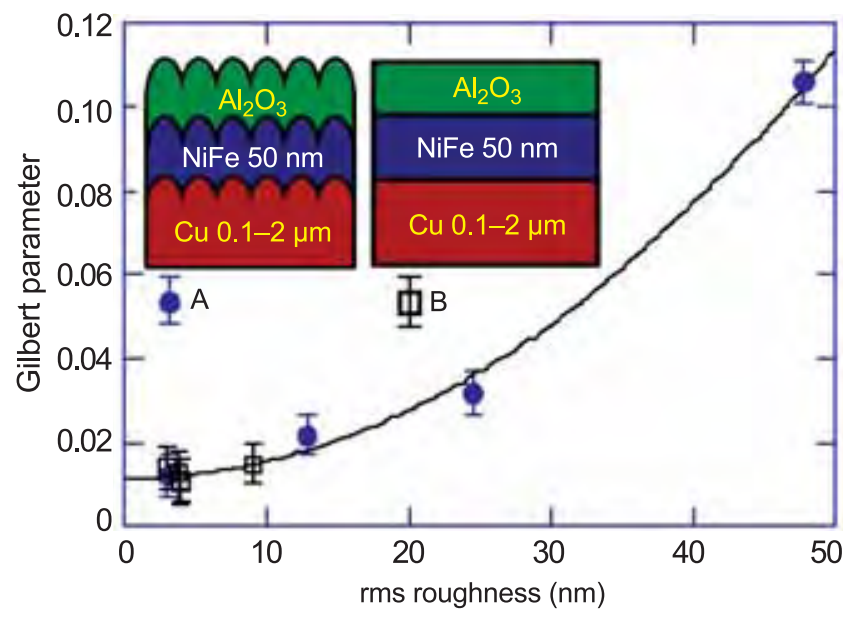

Figure 1: Damping as a function of roughness in Permalloy films on thick $\mathrm{Cu}$ substrates. The quadratic behavior is explained by a model featuring eddy currents that are excited by the roughness-induced stray magnetic fields in the copper.

It is well known that surface affects and interfacial affects are important in nanostuctured materials. This is especially true for magnetic materials, where long-range interactions couple the surface magnetism to the bulk. Figure 1 shows a set of measurement results that show how interfacial properties affect damping in $\mathrm{Ni}_{80} \mathrm{Fe}_{20}$, (Permalloy) on a copper substrate.
The increased damping is due to the excitation of eddy currents by magnetostatic fields associated with the roughness. We have also measured an additional component of interfacial damping that exists for smooth interfaces.

We have started a new effort within this project to develop measurement techniques for characterization of magnetic edges. The edge properties of a magnetic device are critical because they affect the shape anisotropy of a patterned device and the nucleation of vortices in the critical stage for switching. While accepted techniques exist for characterizing bulk materials and interfaces, there are no such techniques available for characterizing the edges of thin film devices.

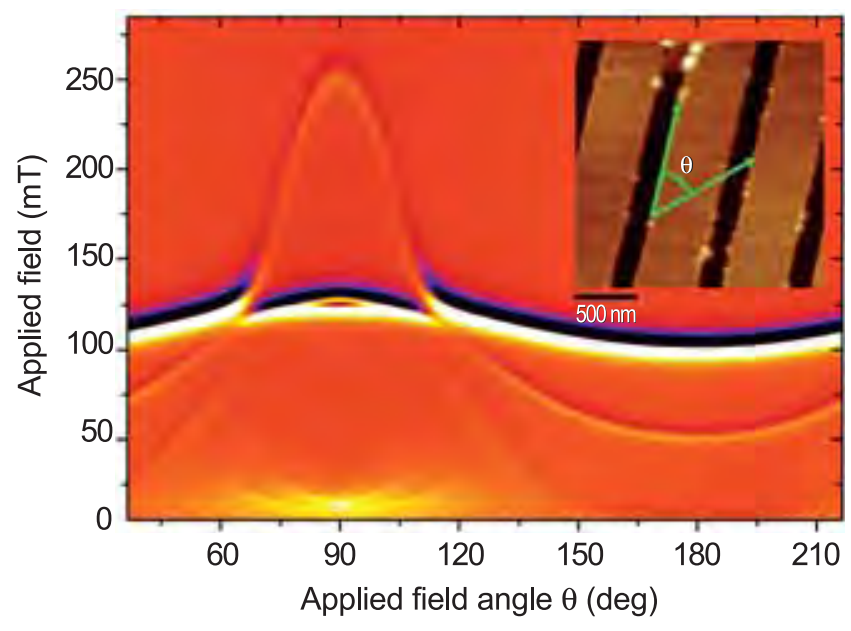

Figure 2: Image composed of 180 FMR spectra measured on an array of Permalloy stripes. An AFM image of the stripes is shown in the inset. At $90^{\circ}$ the applied field is perpendicular to the stripes, and the peak is the resonance of the edge modes.

Although the edge metrology aspect of the project has just started, we have already performed proof-of-concept magnetometry of edge saturation fields and ferromagnetic resonance measurements of localized edge modes (see Figure 2). These measurements are interpreted through corresponding micromagnetic modeling of the static and dynamic behavior of spins near edges. The stage is also set for magnetoresistive measurements that would be more suitable for wafer-level probing of the lithography process.

\section{Contributors and Collaborators}

J. Rantschler, B. Maranville, J. Mallett, T. Moffatt, W. Egelhoff, Jr., A. Chen (Metallurgy Division, NIST); C. Ross, J. Cheng (MIT) 


\section{Materials for Ultra-Low-Field Magnetic Sensors}

Magnetic sensors play a central role in many important technologies ranging from health care to homeland security. A common need among these technologies is greater sensitivity and smaller size. A NIST competence program initiated in 2004 is focusing on developing the metrology that will enable new generations of magnetic sensors to be produced.

\section{William F. Egelhoff, Jr.}

Tn the past decade, small inexpensive magnetic 1 sensors have gained important footholds in a wide variety of technologies. Sales of small inexpensive magnetic sensors have been soaring. However, one relatively neglected aspect of these magnetic sensors has been their sensitivity to very small magnetic fields.

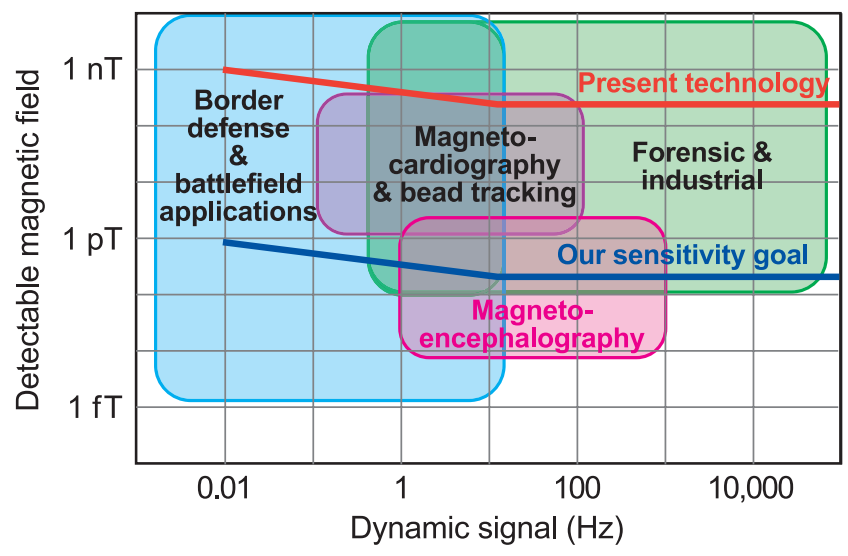

Figure 1: A plot of the sensitivity to very small magnetic fields that is required for various applications as a function of the frequency of the signal to be detected. Also indicated are the limits of present technology for small inexpensive solid-state sensors and the goals of the Competence Program.

Figure 1 indicates, for particular applications, the range of fields that the sensor must be able to detect and the range of frequencies at which the fields appear. For comparison, the Earth's magnetic field is about $10^{4}$ times larger than the top value of Figure 1. The plots of "Present technology" and "Our sensitivity goal" in Figure 1 indicate that deep inroads can potentially be made into important technologies if we can reach our goal.

It should be pointed out that the technique of superconducting quantum interference devices (SQUIDs) can already reach sensitivities corresponding to the bottom values of Figure 1. Unfortunately, SQUIDs are expensive, bulky, and require cryogenic cooling. These factors rule out SQUIDs for all applications except those for which size and cost are not a factor and for which cryogenic cooling is not prohibitively inconvenient.
We are focusing on small inexpensive thin-film magnetic sensors to avoid the disadvantages of SQUIDs. Existing precedents suggest that such sensors could be mass produced at a cost of a few cents each, will have a size measured in microns, and will operate at room temperature. These characteristics make them ideal for the applications indicated in Figure 1.

To achieve our goal we will need magnetic thin films of great sensitivity. The best thin films previously known had susceptibilities of a few thousand. In our first year of work, we have already achieved values above 100 thousand.

One of the prime applications that we may impact is magneto-cardiography, or imaging the beating heart in real time. In principle, magneto-cardiography could give very high quality 3-D images. However, at present, SQUID-based magneto-cardiography is hugely expensive, has poor resolution, and is practiced only at a few research institutions. If small, inexpensive sensors could make high-resolution magneto-cardiography generally available, it would change the practice of cardiology. The following quote arose in a discussion of our plans.

"Real-time magneto-cardiography with $1 \mathrm{~cm}$ resolution would have revolutionary diagnostic impact."

Bob Balaban, Director

National Heart, Lung, and Blood Institute National Institutes of Health

As Figure 1 would suggest, magneto-encephalography may be too demanding an application for the magnetic sensors we envision. However, it is always difficult to forecast the limits on new technologies. The one thing that is very clear is that we have very little competition in this field of research. The companies that currently produce the small, inexpensive magnetic sensors do not have active research and development programs in the area of ultra-low fields. The narrow profit margins for existing products will not support such programs. This situation puts NIST in an ideal position to take the lead and perform a high-risk/high-payoff research program that would not otherwise be undertaken.

\section{Contributors and Collaborators}

R. McMichael, C. Dennis, F. Johnson, B. Maranville, J. Rantschler, A. Shapiro (Metallurgy Division, NIST); J. Unguris (Physics Lab, NIST); D. Pappas, S. Russek (EEEL, NIST) 


\section{A New Type of Antisymmetric Magnetoresistance in Materials with Perpendicular Anisotropy}

When the magnetization reverses direction in a ferro-magnet upon the application of a reversed magnetic field, it usually occurs by the nucleation of local domains with a reversed magnetization vector which subsequently grows to envelop the whole material. The ease (or difficulty) by which such a process occurs is key to determining how useful that material will be for its intended application. The metrology for detecting, measuring, and imaging the process in various classes of materials is being developed in this project. This knowledge is necessary for quick implementation of new magnetic materials for the recording, refrigeration, theft control, and power industries.

\section{Robert D. Shull, Valerian I. Nikitenko, and Alexander J. Shapiro}

$\mathrm{T}$ The simplest way to detect the magnetic state of a material is to sense the magnetic field that surrounds it. However, as magnetic devices are reduced in size, the magnitude and spatial extent of these fields are also reduced. Recently, very sensitive field sensors (e.g., giant (GMR), tunneling (TMR), and colossal (CMR) magnetoresistance detectors) have been developed which are based upon measuring the resistance changes caused by the presence of small magnetic fields. In all these devices, the resistance changes are all symmetric with respect to the sign of the magnetic field. We report here the discovery of a new magnetoresistance effect in a multilayer which changes sign with changes in the direction of the magnetic field. Furthermore, the phenomenon is found to be caused

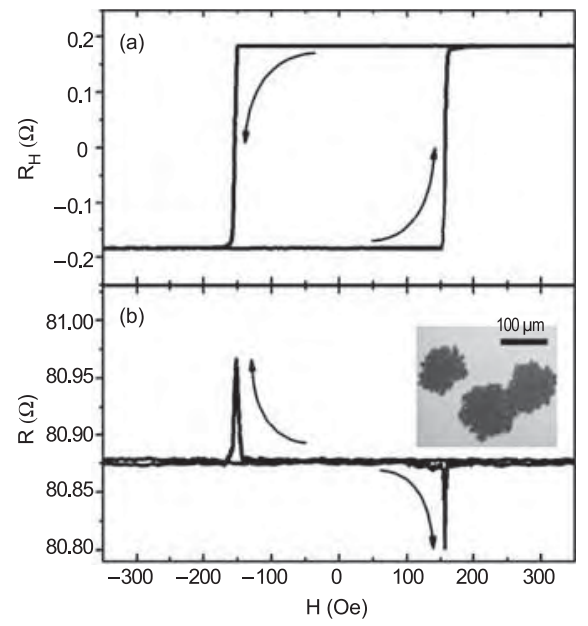

Figure 1: Arrows denote the sequence of measurements; inset shows the domain patterns correlated with magnetization reversal at the $M R$ and $R_{H}$ peaks. by the presence of domain walls running perpendicular to the current direction.

Figure 1 shows the antisymmetric Hall resistance $\left(\mathrm{R}_{\mathrm{H}}\right)$ and in-line resistance $(\mathrm{R})$ for a $[\mathrm{Co}(0.6 \mathrm{~nm}) /$ $\mathrm{Pt}(1 \mathrm{~nm})]_{4}$ multilayer as the magnetic field $(\mathrm{H})$ is cycled between positive and negative fields. Note that $\Delta \mathrm{R}(\mathrm{H})=-\Delta \mathrm{R}(-\mathrm{H})$ in (b), indicating the magnetoresistance (MR) is negative when switching from negative fields to positive fields while it is positive when switching from positive fields to negative fields, is similar to the Hall effect but quite different from normal MR phenomena.

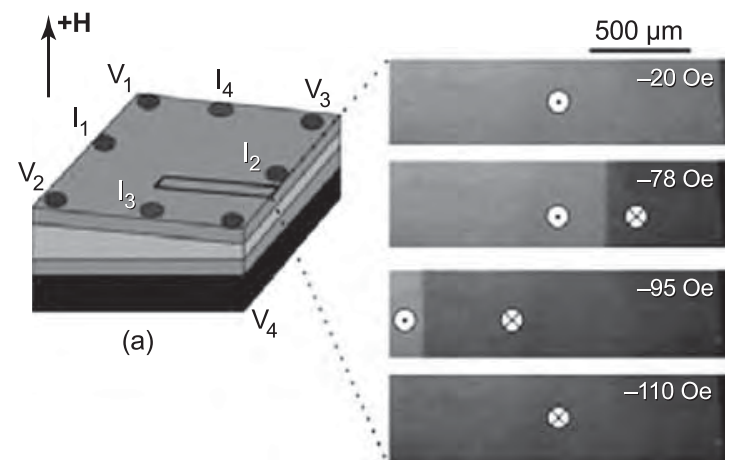

(b)

Figure 2: Wedge sample showing: (a) leads for measuring current, I, and voltage, $V$; (b) domain positions as a function of field.

By the controlled creation of a single domain wall running perpendicular to the current direction (shown in Figure 2), the antisymmetric magnetoresistance has been shown to be due to the presence of such a domain wall. This configuration was created by preparing a trilayer containing a wedge-shaped Co layer (varying in thickness from $0.3 \mathrm{~nm}-0.6 \mathrm{~nm}$ ) sandwiched by $3 \mathrm{~nm}$ thick Pt layers. We had earlier found in such a trilayer that one could create such a single domain wall running perpendicular to the wedge at a position along the wedge determined by the magnitude of the reversed field applied, as shown in Figure 2(b).

The unusual magnetoresistance effect is due to circulating currents created at the domain wall due to the opposite Hall voltages on either side of the wall. The results, which were published in Phys. Rev. Letters 94, 17203 (2005), provide much more flexibility to the circuit designers and may point the way to creating double pole magnetic switches.

\section{Contributors and Collaborators}

X. Cheng, S. Urazhdin, O. Tchernyshyov, C. Chien (Johns Hopkins University) 


\section{Nanostructure Fabrication Processes: Surface \& Growth Stress During Thin Film Electrodeposition}

The adsorption of species onto a surface alters the surface stress since the local interaction of each adsorbate alters the bond strength between neighboring atoms on the surface. Our immediate focus is to use surface stress to examine substrateadsorbate interactions of various molecules for self-assembled monolayers (SAM). A natural extension of this work is to examine the surface stress associated with adsorbate-adsorbate lateral interactions. This could lead to a general in situ metrology for control of surfactants and adsorbatebased surface modification for a variety of molecular electronics and sensor applications.

\section{Gery R. Stafford and Carlos Beauchamp}

urface stresses arise because the atomic configuration of atoms at a surface is different from the bulk. Interior atoms exert a stress on the surface atoms, i.e., the surface stress, which moves them out of the positions they would occupy in a bulk crystal configuration. The adsorption of species onto the surface can be expected to alter the surface stress since the local interaction of each adsorbate will alter the bond strength between neighboring atoms on the surface. An understanding of adsorbate-induced surface stress is critical to emerging technologies such as molecular electronics, nanostructure fabrication, and chemical/biological sensors.

We have established a Class II (1 mW) HeNe optical bench dedicated to in situ measurement of surface and growth stress during electrochemical processing using the wafer curvature method. Measurements are made on a borosilicate glass cantilever onto which $250 \mathrm{~nm}$ of $\mathrm{Au}$ is evaporated. The curvature of the substrate is monitored while in solution and under potential control by reflecting a HeNe laser off the glass/metal interface, through a series of mirrors, and onto a position-sensitive detector. The relationship between force exerted onto the cantilever by the electrochemical processes occurring at the Au-solution interface and the radius of curvature of the cantilever is given by Stoney's equation. Forces on the order of $0.008 \mathrm{~N} / \mathrm{m}$ (23 km radius of curvature) can be resolved. This is sufficient to study the adsorption of molecular monolayers onto the electrode surface. As a demonstration, we have measured the surface stress associated with reversible monolayer adsorption of both sulfate and chloride ions onto the Au surface from aqueous solutions.

Figure 1 shows the linear sweep voltammetry (three consecutive transients taken 5 minutes apart) as well as the associated surface stress of a (111)-textured $\mathrm{Au}$

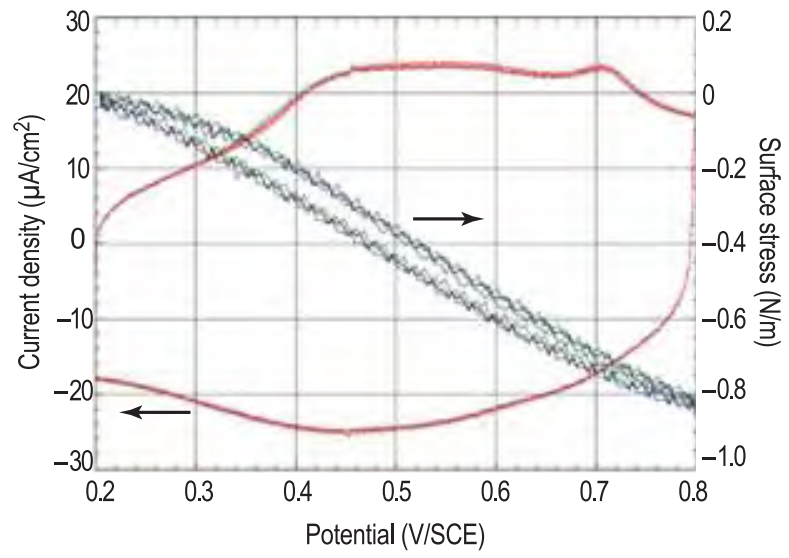

Figure 1: Linear sweep voltammetry and surface stress associated with sulfate adsorption on (111)-textured $\mathrm{Au}$ in $1.0 \mathrm{~mol} / \mathrm{L} \mathrm{H} 2 \mathrm{SO} 4$.

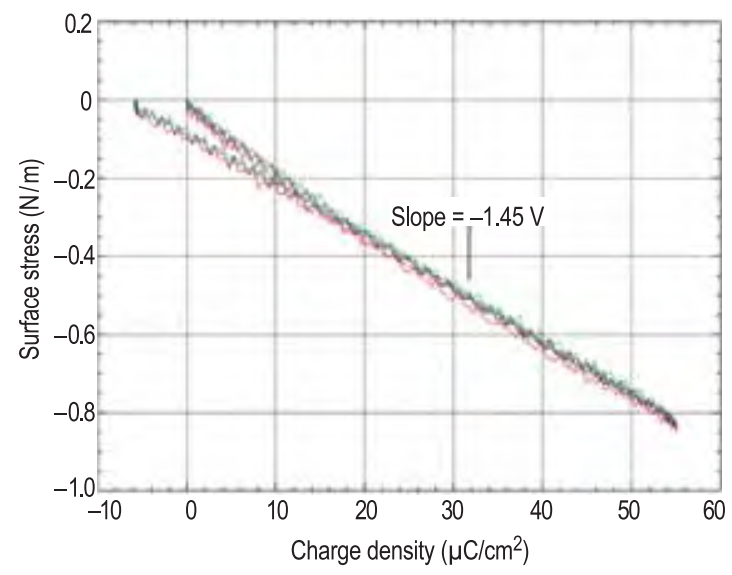

Figure 2: Surface stress vs. charge density associated with sulfate adsorption on (111)-textured Au in $1.0 \mathrm{~mol} / \mathrm{L} \mathrm{H} 2 \mathrm{SO} 4$.

electrode in $1.0 \mathrm{~mol} / \mathrm{L} \mathrm{H}_{2} \mathrm{SO}_{4}$. As the electrode potential is swept in the positive direction from $0.2 \mathrm{~V}$, a positive current is measured as the result of sulfate ion adsorption onto the Au surface. As negative charge is removed from the electrode with potential increase, the surface stress moves in the negative (compressive) direction, consistent with charge redistribution models that appear in the literature. The adsorption of electronegative species such as sulfate ion increases the compressive trend in the surface stress by removing additional charge from the Au surface. Figure 2 is a plot of the surface stress vs. charge density for the sulfate adsorption/desorption process. The data suggest that the slope of the stress-charge curve provides a measure of adsorbate strength that can be used to quantify adsorbate-substrate interactions.

\section{Contributors and Collaborators}

U. Bertocci (Metallurgy Division, NIST); C. Zangmeister (Process Measurements Division, NIST) 


\section{Multiscale Modeling of Quantum Dots in Semiconductors}

\begin{abstract}
A computationally efficient multiscale model has been developed for a quantum dot in a semiconductor. The model links the subnano, nano, and macro length scales by integrating the powerful techniques of molecular dynamics, lattice-statics, and continuum Green's functions. The model is applied to Ge quantum dots of realistic sizes up to $8 \mathrm{~nm}$ in Si. The topography of a free surface of Si, containing a buried quantum dot, is calculated. This surface can be measured and used to characterize the $Q D$. The model can also predict the formation of arrays of quantum dots and can be a useful tool for strain engineering of quantum dots.
\end{abstract}

Vinod K. Tewary and David T. Read

\section{Technical Description}

Currently there is a strong interest in modeling the mechanical characteristics of quantum dots (QDs) in semiconductors because of their potential application in powerful new devices like huge memory systems, ultra low threshold lasers, and quantum computers. A QD has to be modeled at the following scales:

(i) the core region (sub-nanometer) where the nonlinear effects may be significant; (ii) the region of the host solid around the QD (nanometer); and (iii) free surfaces and interfaces in the host solid (macro). Modeling is needed for interpreting measurements and design of new devices. A multiscale model is especially useful for strain engineering of QDs and their arrays.

A QD causes lattice distortion in the host solid which is manifested as strain and a displacement field throughout the solid. Strains and a displacement field at a free surface can be measured and used to characterize the QD. A strain field determines the elastic energy of the system and is mainly responsible for the formation of arrays of QDs. The strain and displacement field are essentially a continuum-model parameter whereas the lattice distortions are discrete variables that must be calculated by using a discrete lattice theory. Hence one needs a multiscale model that relates the discrete lattice distortions at the microscopic scale to a macroscopic parameter such as strain.

We have developed a computationally efficient multiscale model that links the length scales from sub-nano to macro and can be used on an ordinary desktop computer. The model integrates classical molecular dynamics (MD) with Green's functions (GF). We use MD at the core of the QD to account for the nonlinear effects and the lattice-statics GF, G, near the quantum dots which reduces asymptotically to the continuum GF near a free surface. The displacement field in this model containing $\mathrm{N}$ atoms is given by:

$$
\mathbf{u}(\mathbf{l})=(1 / \mathrm{N}) \Sigma_{\mathbf{k}} \mathbf{G}(\mathbf{k}) \mathbf{F}(\mathbf{k}) \exp (\mathrm{ik} . \mathbf{l})
$$

where $\mathbf{l}$ is a lattice site, $\mathbf{k}$ is a reciprocal space vector, and $\mathbf{F}(\mathbf{k})$ is the Kanzaki force which is calculated by using MD. For large l, the above equation reduces asymptotically to the macroscopic continuum while the discrete lattice effects are retained in $\mathbf{F ( k )}$. Thus, our model is truly multiscale as it seamlessly links the discrete atomistic effects in $\mathbf{F}(\mathbf{k})$ to macroscopic scales through the GF and directly relates microscopic lattice distortion at nanoscale to measurable macroscale parameters.

\section{Accomplishments}

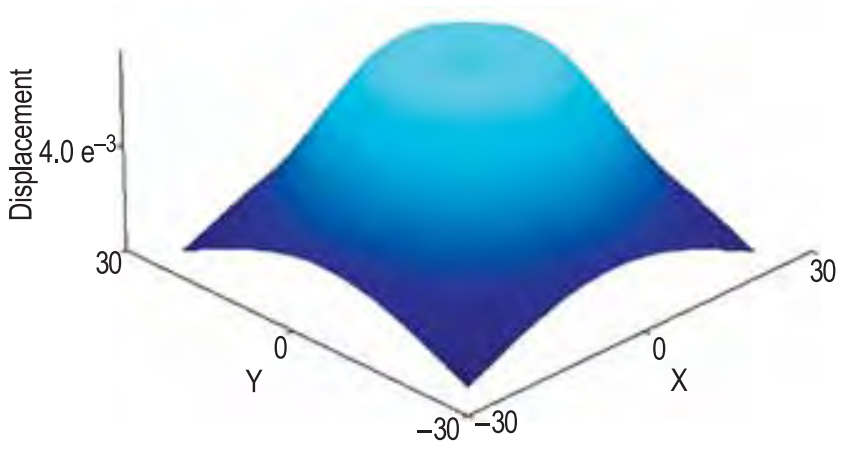

Figure 1: Topography of a free surface in Si due to a buried $1.1 \mathrm{~nm}$ Ge QD in Si.

We are now able to model quantum dots of realistic sizes, up to about 8 nanometers, on a desktop computer. To model such a large QD, it is necessary to include at least a million atoms in the host lattice. An attempt to model such a large system using only MD would require a huge computational effort and involve somewhat arbitrary assumptions for relating discrete lattice distortion to continuum parameters. We have calculated surface strains and surface topography (Figure 1) which can be measured and used to characterize the QDs. Note the local minimum at $(0,0)$. A similar minimum occurs in the strain energy for certain QDs. The position of the minimum is a possible favorable location for the nucleation of a new QD.

\section{Contributors and Collaborators}

R.R. Keller (Materials Reliability Division, NIST); B. Yang (Florida Tech); R. Pandey (Michigan Tech University) 


\section{Brillouin Light Scattering: Dynamic Elastic and Magnetic Properties of Nanostructures}

Brillouin light scattering can be used to provide information on acoustic waves and spin waves at gigahertz frequencies in nanometric thin-film materials and devices. During FY05, we focused on developing the metrology and models for characterizing waves in several material systems, including nanoimprinted polymers and ferromagnetic thin films with nanoscale edge effects.

\section{Ward Johnson, Sudook Kim, and Colm Flannery}

$\mathrm{B}$ rillouin light scattering (BLS) is an experimental technique that measures the intensity of spectral components of light that is inelastically scattered by vibrational waves (acoustic phonons) or spin waves (magnons) in a material. Fabry-Perot interferometric techniques are used to acquire accumulated spectra through repeated mechanical sweeping of the etalon spacing.

In the Materials Reliability Division, BLS is being pursued as a technique for characterizing dynamic elastic and magnetic properties of a variety of materials and devices with nanoscale dimensions. During FY05, research on elastic waves focused on nanoimprinted polymeric lines (highlighted below), carbon nanotubes, and polymeric membranes. Research on spin waves focused on magnon-magnon interactions in $\mathrm{Ni}_{81} \mathrm{Fe}_{19}$, modes localized near edges of ferromagnetic thin-film structures, and modes excited in spin-momentumtransfer devices.

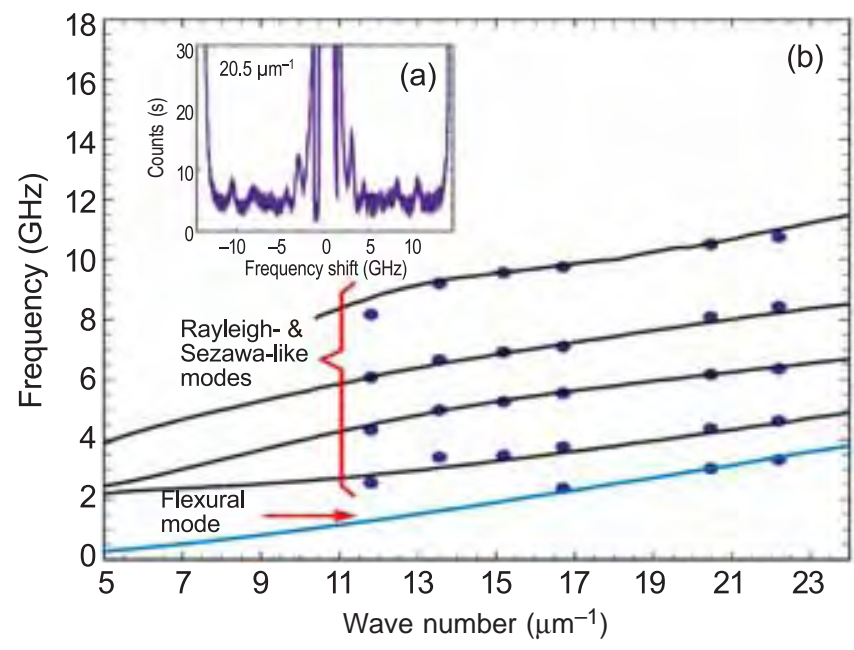

Figure 1: (a) BLS spectrum for PMMA nanolines; (b) Measured dispersion curves (blue circles), calculations for a uniform film (black curves), and Lamb-wave calculations (blue curve).

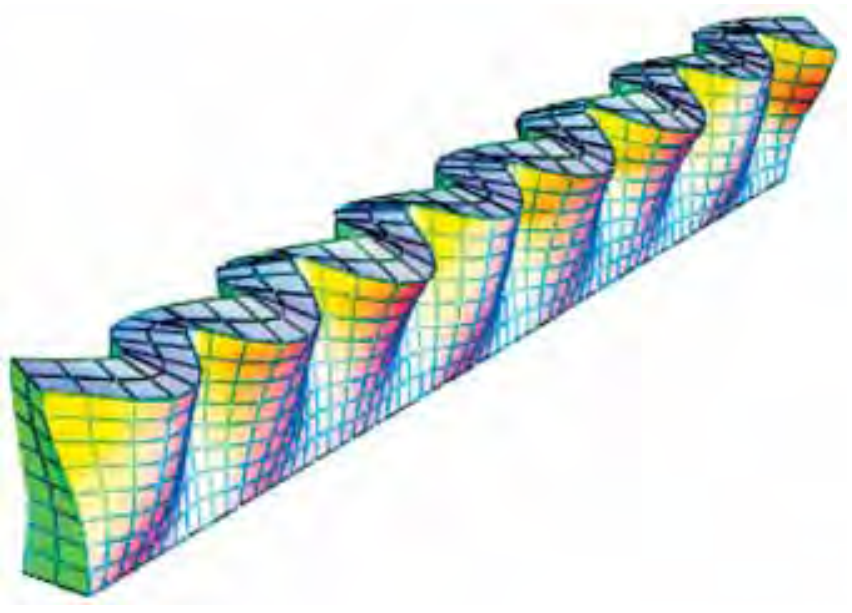

Figure 2: Finite-element calculation of displacements of a flexural mode of a PMMA nanoline.

Research on nanoimprinted polymers was pursued in collaboration with the NIST Polymers Division, Colorado State University, and the University of Akron. The goal of this work is to develop experimental and analytical methods for characterizing elastic properties, which are expected to deviate from bulk properties when one or more dimensions of a nanoline are less than a few tens of nanometers. Figure 1 shows a typical BLS spectrum from an array of imprinted polymethyl-methacrylate (PMMA) nanolines and plots of dispersion curves from a series of spectra obtained at various scattering angles. The general character of the observed modes, except for the lowest, is determined through Fresnel-Adler calculations (black lines) for a uniform film with a thickness equal to the height of the nanolines plus the thickness of a residual PMMA layer beneath the lines. The lowest-frequency set of points arises from transverse flexural modes of the lines. The identification of these modes is based partly on the correspondence of the data with calculations of the lowest order flexural modes (antisymmetric Lamb waves) of a plate with thickness equal to the width of the nanolines (blue curve in Figure 1). Finite-element calculations* have been employed to provide detailed information on the vibrational displacements, such as those of the flexural mode shown in Figure 2.

\section{Contributors and Collaborators}

C. Soles, C. Stafford (Polymers Division, NIST); R. McMichael (Metallurgy Division, NIST); P. Kabos, W. Rippard, S. Russek (Electromagnetics Division, NIST); P. Heyliger, P. Krivosik (Colorado State University); R. Hartschuh, A. Sokolov (University of Akron) 


\section{Materials for Electronics}

The U.S. electronics industry faces strong international competition in the manufacture of smaller, faster, more functional, and more reliable products. Many critical challenges facing the industry require the continual development of advanced materials and processes. The NIST Materials Science and Engineering Laboratory (MSEL) works closely with U.S. industry, covering a broad spectrum of sectors including semiconductor manufacturing, device components, packaging, data storage, and assembly, as well as complementary and emerging areas such as optoelectronics and organic electronics. MSEL has a multidivisional approach, committed to addressing the most critical materials measurement and standards issues for electronic materials. Our vision is to be the key resource within the Federal Government for materials metrology development and will be realized through the following objectives:

- Develop and deliver standard measurements and data for thin film and nanoscale structures;

- Develop advanced measurement methods needed by industry to address new problems that arise with the development of new materials;

- Develop and apply in situ as well as real-time, factory floor measurements for materials and devices having micrometer to nanometer scale dimensions;

- Develop combinatorial material methodologies for the rapid optimization of industrially important electronic and photonic materials;

- Provide fundamental understanding of the divergence of thin film and nanoscale material properties from their bulk values;

- Provide fundamental understanding, including first principles modeling, of materials needed for future nanoelectronic devices.

The NIST/MSEL program consists of projects led by the Metallurgy, Polymers, Materials Reliability, and Ceramics Divisions. These projects are conducted in collaboration with partners from industrial consortia (e.g., SEMATECH), individual companies, academia, and other government agencies. The program is strongly coupled with other microelectronics programs within the government such as the National Semiconductor Metrology Program (NSMP). Materials metrology needs are also identified through the International Technology Roadmap for Semiconductors (ITRS), the International Packaging Consortium (IPC) Roadmap, the IPC Lead-free Solder Roadmap, the National Electronics Manufacturing Initiative (NEMI) Roadmap, the Optoelectronics Industry Development Association (OIDA) Roadmap, and the National Magnetic Data Storage Industry Consortium (NSIC) Roadmap.
MSEL researchers from each division have made substantial contributions to the most pressing technical challenges facing industry, from new fabrication methods and advanced materials in the semiconductor industry, to low-cost organic electronics, and to novel classes of electronic ceramics. Below are just a few examples of MSEL contributions over the past year.

\section{Advanced Gate Dielectrics}

To enable further device scaling, the capacitive equivalent thickness (CET) of the gate stack thickness must be $0.5 \mathrm{~nm}$ to $1.0 \mathrm{~nm}$. This is not achievable with existing $\mathrm{SiO}_{2}$ /polcrystalline $\mathrm{Si}$ gate stacks. High dielectric constant gate insulators are needed to replace $\mathrm{SiO}_{2}$, and metal gate electrodes are needed to replace polycrystalline Si. Given the large number of possible materials choices for the gate dielectric/substrate and gate dielectric/metal gate electrode interfaces, the MSEL Ceramics Division is establishing a dedicated combinatorial film deposition facility to study the complex interfacial interactions. This same methodology is applicable to a wide variety of problems in the electronic materials field.

\section{Advanced Lithography}

Lithography is the key enabling technology for the fabrication of advanced integrated circuits. As feature sizes decrease to sub-65 nm length scales, challenges arise because the image resolution and the thickness of the imaging layer approach the dimensions of the polymers used in the photoresist film. Unique high-spatial resolution measurements are developed to identify the limits of materials and processes for the development of photoresists for next-generation lithography.

\section{Advanced Metallization}

As the dimensions of copper metallization interconnects on microelectronic chips decrease below $100 \mathrm{~nm}$, control of electrical resistivity becomes critical. The MSEL Metallurgy Division is developing seedless deposition methods that will simplify thin-film processing and result in film growth modes that increase trench filling, thus lowering interconnect resistivity.

\section{Mechanical Reliability of Microchips}

One of the important ITRS challenges is to achieve effective control of the failure mechanisms affecting chip reliability. Detection and characterization methods for dimensionally constrained materials will be critical to the attainment of this objective. Scientists in the MSEL Materials Reliability Division are addressing this issue by focusing on electrical methods capable of determining the thermal fatigue lifetime and mechanical strength of patterned metal film interconnects essential to microchips.

Contact: Martin L. Green (Ceramics Division), Eric K. Lin (Polymers Division) 


\section{Multifunctional Electronic Ceramics}

\begin{abstract}
Materials which exhibit exploitable and coupled responses to multiple external fields (electric, magnetic, etc.) present entirely new design opportunities for multifunctional, miniaturized devices such as sensors or actuators. The goal of this project is to establish transferable engineering principles for multi- and single-phase electronic ceramics which exhibit a functional response by one constituent phase/subsystem that is generated by the response of another phase/subsystem to an external field.
\end{abstract}

\section{Igor Levin, Julia Slutsker, and Terrell A. Vanderah}

$\mathrm{T}$ he development of next-generation multifunctional devices now includes a search for materials exhibiting simultaneous magnetic, electronic, and/or photonic responses (e.g., magnetic semiconductors, magnetic superconductors, multiferroics, etc.). Multiferroic materials display a coexistence of ferroelectric and ferromagnetic responses and have attracted particular interest for several novel device applications including memories, sensors, and actuators. Self-assembled, epitaxial heterophase nanostructures consisting of both ferromagnetic and ferroelectric phases represent one promising class of multiferroics. The strong magnetoelectric coupling obtained in these materials is attributed to the nanoscale distribution of the phases, which facilitates highly efficient elastic interactions and a strong magnetic response to an electric field (or vice versa) via magnetostriction and the piezoelectric effect. The same elastic interactions also control the self-assembly of the component phases, so that the architecture and the scale of the nanostructures can be predicted and controlled by manipulating the stress state of the film.

We analyzed the affect of stress conditions on the morphologies of epitaxial, self-assembled nanostructures

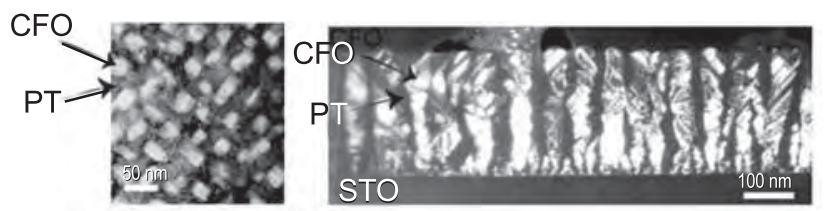

(001) STO

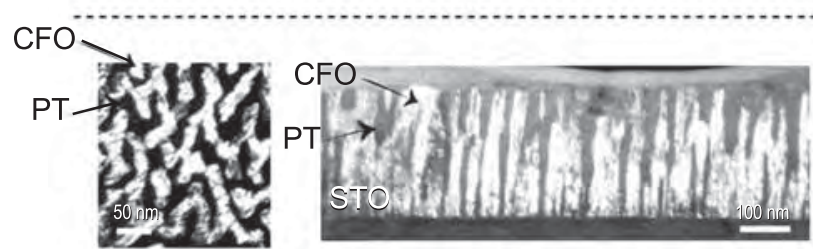

(110) STO

Figure 1: Plane view and cross-sectional images of the $0.33 \mathrm{CoFe}_{2} \mathrm{O}_{4}-0.67 \mathrm{PbTiO}_{3}$ nanostructures grown epitaxially on (001) and (110) $\mathrm{SrTiO}_{3}$.

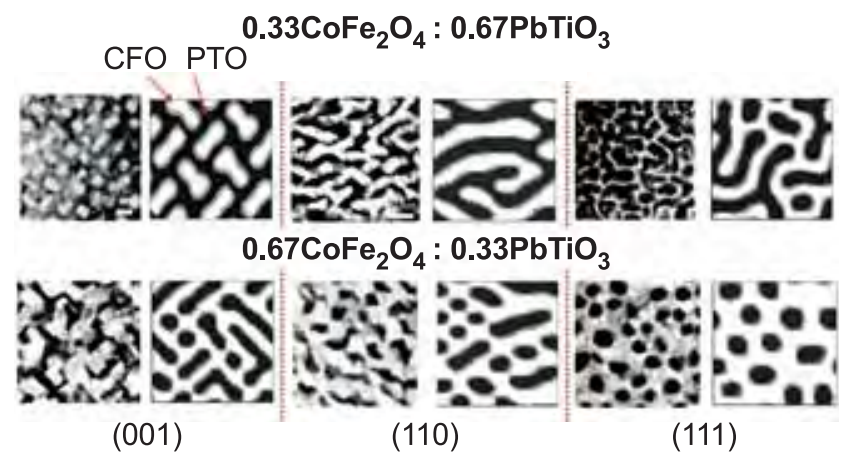

Figure 2: Plane view of $\mathrm{CoFe}_{2} \mathrm{O}_{4}-\mathrm{PbTiO}_{3}$ nanostructures grown epitaxially on (001), (110), and (111) $\mathrm{SrTiO}_{3}$. For each composition and orientation, the experimentally observed structure is shown on the left and the simulation is shown on the right.

using $\mathrm{PbTiO}_{3}-\mathrm{CoFe}_{2} \mathrm{O}_{4}$ thin films. The two-phase nanostructures were grown on single-crystal $\mathrm{SrTiO}_{3}$; the strain conditions were varied by deposition on differently oriented substrates. Regardless of orientation, the nanostructures consisted of vertical columns of ferromagnetic $\mathrm{CoFe}_{2} \mathrm{O}_{4}$ dispersed in a ferroelectric $\mathrm{PbTiO}_{3}$ matrix, or vice versa (Figure 1). However, the morphologies of these columns and their spatial arrangements exhibited a marked dependence on substrate orientation. Phase field modeling of these nanostructures, which assumed an equilibrium corresponding to the minimum of elastic and interfacial energies at a given phase fraction, succeeded in reproducing the morphological differences (Figure 2). The modeling confirmed that these differences are related to elastic anisotropy in the film. Our results, which demonstrate that the architecture of self-assembled multiferroic nanostructures can indeed be controlled by a careful choice of the stress conditions, open a tantalizing opportunity for the rational design of self-assembled, multifunctional nanostructures.

Research on bulk single-phase materials included phase equilibria studies of the $\mathrm{Bi}_{2} \mathrm{O}_{3}-\mathrm{Fe}_{2} \mathrm{O}_{3}-\mathrm{Nb}_{2} \mathrm{O}_{5}$ and $\mathrm{Bi}_{2} \mathrm{O}_{3}-\mathrm{Mn}_{2} \mathrm{O}_{3}-\mathrm{Nb}_{2} \mathrm{O}_{5}$ systems. Surprisingly, both systems feature extensive pyrochlore-type phase fields at compositions requiring mixing of the magnetic ions with far larger $\mathrm{Bi}^{3+}$ ions on the A-sites, in apparent violation of traditional substitutional rules. The pyrochlore phases exhibited relative permittivities $\sim 150$, and were readily deposited on $\mathrm{Si}$ as crystalline thin films using pulsed laser deposition. Although the multiferroic phase $\mathrm{BiFeO}_{3}$ was found to participate in ambient-pressure phase assemblages, $\mathrm{BiMnO}_{3}$ did not, and was not stabilized by the presence of $\mathrm{Nb}^{5+}$.

\section{Contributors and Collaborators}

P.K. Schenck (Ceramics Division, NIST); V. Provenzano (Metallurgy Division, NIST); J. Li, A.L. Roytburd (University of Maryland) 


\section{Spectroscopy, Diffraction, and Imaging of Electronic Materials}

We are working to develop, establish, and provide synchrotron-based metrology, including instrumentation and technical expertise, for spectroscopy, diffraction, and imaging techniques applicable to the study of nanoscale and other phenomena that are important in the design, application, and performance of electronic materials.

\section{Joseph C. Woicik}

$\mathrm{T}_{\mathrm{p}}^{\mathrm{h}}$ he epitaxial growth of oxides on silicon opened the possibility of incorporating many of their unique electronic properties into silicon device technology. We have studied the epitaxy and lattice expansion of $\mathrm{SrTiO}_{3}$ thin films grown coherently on $\mathrm{Si}(001)$ by kinetically controlled sequential deposition. Coherent growth is achieved by repetition of the deposition sequence that includes a low-temperature and high-oxygen partial-pressure step followed by a high-temperature and low-oxygen partial-pressure step, thereby suppressing the detrimental oxidation of the silicon substrate.

Unlike films grown by more traditional molecular-beam-epitaxy (MBE) methods, these films are found to have an in-plane lattice constant that is indistinguishable from the silicon substrate, an out-of-plane lattice constant that is expanded by an amount twice that predicted by the bulk elastic

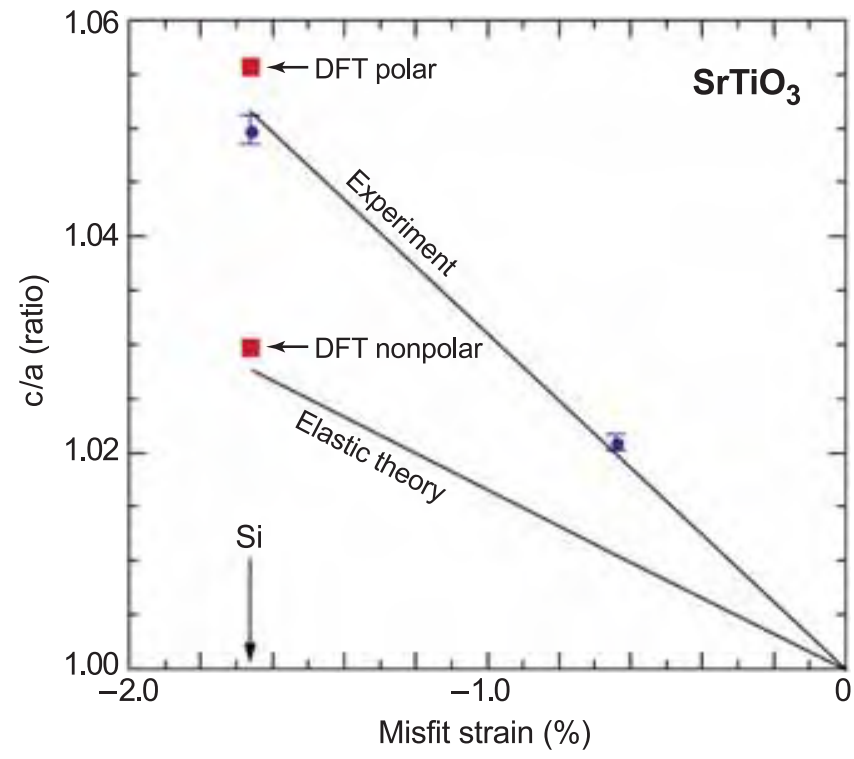

Figure 1: Measured c/a ratio for $5 \mathrm{ML}$ and $10 \mathrm{ML}$ films as a function of in-plane lattice mismatch. Also shown are the predictions of elastic theory and density functional theory (DFT). constants of $\mathrm{SrTiO}_{3}$, and a critical-thickness behavior beyond $\sim 2 \mathrm{~nm}$ (5 unit cells or 5 monolayers (ML's)).

The experimentally determined $c / a$-ratio as a function of in-plane misfit strain is shown in Figure 1, for both $5 \mathrm{ML}$ and $10 \mathrm{ML}$ films. The experimental results are compared to the results of density functional theory for the $c / a$ ratio of a $5 \mathrm{ML}$ film in coherent registry with the silicon substrate as shown in Figure 2. The difference between the two structures in Figure 2 is the presence of $\mathrm{OH}$ adsorbates on the surface and oxygen vacancies at the interface, as revealed by high-resolution x-ray photoelectron spectroscopy for films that have been exposed to air. (a)

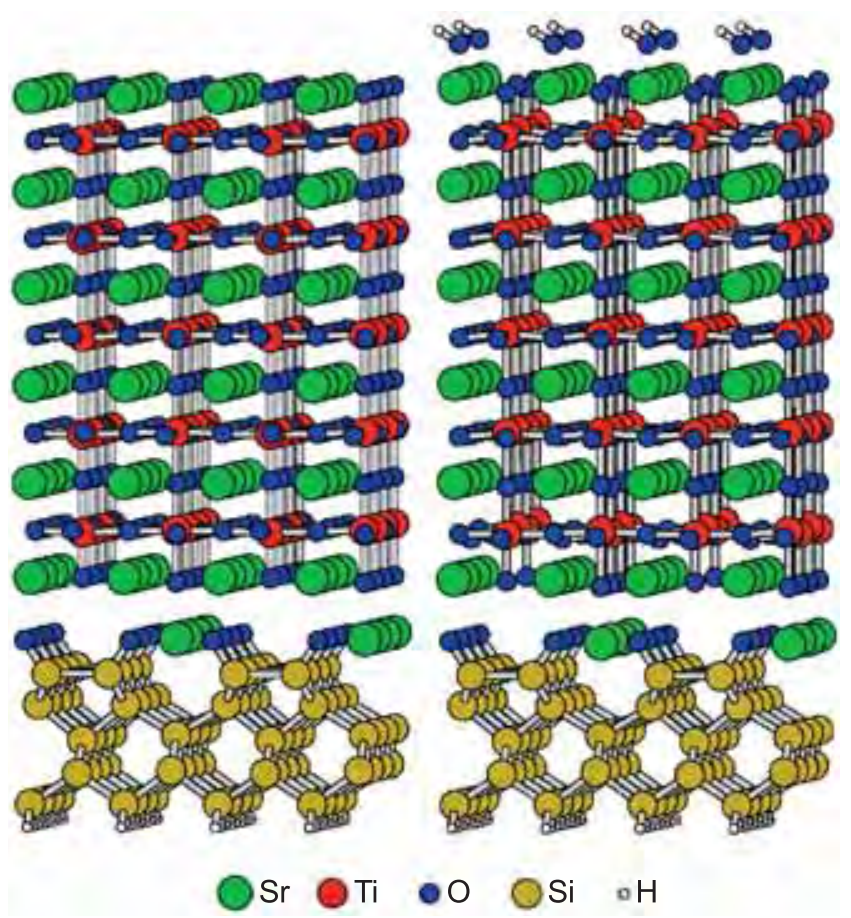

Figure 2: Structure of the ideal $5 \mathrm{ML} \mathrm{SrTiO}_{3} / \mathrm{Si}(001)$ system (a) and the system with $\mathrm{O}$ vacancies and $\mathrm{OH}$ adsorbates (b). Note the ferroelectric polarization in (b) but not in (a).

This energetically favorable interfacial-defect/ surface-charge structure compensates the ferroelectric depolarization field and allows the ferroelectric polarization in these ultra-thin films that is confirmed by Ti K-edge x-ray absorption fine-structure measurements.

\section{Contributors and Collaborators}

H. Li (Motorola Labs); P. Zschack, E. Karapetrova (UNICAT); P. Ryan (Ames Lab); C.R. Ashman, C.S. Hellberg (NRL); A. Allen, D. Black, M. Green, I. Levin (Ceramics Division, NIST) 


\section{Theory and Modeling of Electronic Materials}

\begin{abstract}
Optimizing the properties of electronic materials requires fundamental understanding of the origin of their useful properties and computational tools that connect atomic-scale knowledge obtained from first-principles calculations to properties that emerge at larger length scales. We are developing first-principles based modeling tools for calculating the physical properties of materials as a function of chemical ordering on different length scales and how these properties respond to changes in the environment (temperature, pressure, electric field, etc.). Such techniques have applications in a variety of areas, including dielectrics, dilute spin glass magnets, light-emitting diodes, multiferroics, phase diagrams, piezoelectrics, semiconductors, and spectroscopy.
\end{abstract}

\section{Eric J. Cockayne, Richard J. Wagner, and Benjamin P. Burton}

$\mathrm{U}$ nderstanding and predicting the physical properties of solid solutions is a difficult problem in general. Even in the simplest case of a harmonic crystal, the vibrational energy as a function of atomic arrangement can have a strong affect on the phase diagram. The calculated maximum temperature for the miscibility gap in $\mathrm{NaCl}-\mathrm{KCl}$ is reduced by about $50 \%$ when vibrational entropy is included, and the agreement with the experimental phase diagram is much improved. Many systems with useful electromechanical properties, such as $\mathrm{PbMg}_{1 / 3} \mathrm{Nb}_{2 / 3} \mathrm{O}_{3}(\mathrm{PMN})$, the relaxor ferroelectric that is a constituent of the ultrahigh piezoelectric compound $\mathrm{PMN}-\mathrm{PbTiO}_{3}$, are highly anharmonic. Effective Hamiltonian $\left(\mathrm{H}_{\text {eff }}\right)$ techniques have been developed to simplify the modeling of these systems, but the effect of chemical disorder on the lattice dynamics of solid solutions is not well understood. We published a methodology, based on maximum localization, for automatically determining the appropriate $\mathrm{H}_{\mathrm{eff}}$ basis for solid solutions and showed that it correctly reproduces the phonon density of states for low-frequency phonons. In PMN, these phonons were determined to be of two types: those involving displacements of $\mathrm{Pb}$, and those involving rotations of oxygen octahedra.

Experiments suggest that the relaxor behavior in $\mathrm{PbSc}_{1 / 2} \mathrm{Nb}_{1 / 2} \mathrm{O}_{3}$ (PSN) is associated with nanoscale chemically ordered regions in a disordered matrix. We performed a molecular dynamics simulation of a PSN $\mathrm{H}_{\mathrm{eff}}$ on a 320000 atom cell. Polarization fluctuations are much larger in the chemically ordered regions,

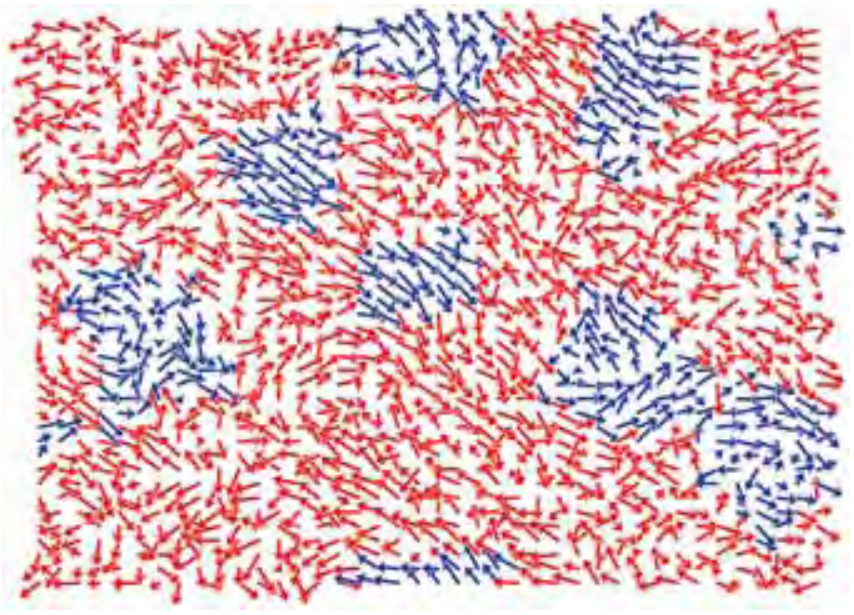

Figure 1: Snapshot of local polarization in PSN model showing larger polarization and polarization correlations in the chemically ordered regions (blue) than in the disordered matrix (red).

which thus dominate the dielectric behavior of PSN near its dielectric peak. This work demonstrates the importance of $\mathrm{H}_{\text {eff }}$ methods that allow us to make a detailed investigation of the effects of nanoscale chemical ordering on dielectric properties.

The semiconductor industry is interested in $\mathrm{HfO}_{2}$ as an alternate gate dielectric material. Experiments typically show significant numbers of defects in $\mathrm{HfO}_{2}$. First principles calculations show that $\mathrm{O}$ vacancies are most stable on the 4-fold coordinated $\mathrm{O}$ site and that $\mathrm{HfO}_{2}$ remains insulating with both a neutral and a 2+ charged vacancy. Work is underway to determine how each kind of vacancy affects the dielectric properties.

Quantitative modeling of mechanical behavior at the nanoscale requires connecting the large-scale elastic displacement fields experienced by the whole device to the small-scale atomistic regions where bond breaking can mark the initiation of device failure through fracture or plastic deformation. We are working to develop multiscale models for connecting the microscopic applied load in a nanoindentation experiment to the initiation of the first broken bond. The sample is a bulk Al single crystal with a <111> surface.

\section{Contributors and Collaborators}

S. Tinte, I. Levin (Ceramics Division, NIST); L. Levine, F. Terrazza (Metallurgy Division, NIST); A. Chaka (CSTL, NIST); A. van de Walle, M. Astar (Northwestern University); D.J. Singh (ORNL); W. Kleeman (University of Duisberg); U.V. Waghmare (J. Nehru Center for Advanced Scientific Research) 


\section{Advanced Materials for Energy Applications}

Fundamental understanding, unique characterization facilities, and standardized materials and data form the technological basis for advances in materials for energy-related technologies. We are working to develop the metrology required to relate properties and performance of energy materials to processing/manufacturing routes via understanding the roles of chemistry, phase relations, and microstructure, to establish a sound physical basis for material system design.

\section{Winnie Wong-Ng, Andrew J. Allen, Daniel A. Fischer, and Lawrence P. Cook}

\begin{abstract}
A challenge for the U.S. economy in the new millennium is for both emerging and mature industries to provide environmentally friendly, inexpensive, efficient, compact, and cutting-edge synergistic technologies for energy conversion, distribution, and storage applications. This project aims to facilitate commercialization of energy-related technologies by addressing various near-term and long-term materials issues.
\end{abstract}

The proliferation of portable telecommunication devices, computer equipment, and hybrid electric vehicles has created a substantial interest in manufacturing rechargeable Li-ion batteries that are less expensive, non-toxic, durable, and small in size and weight. The electronic structure of the electrode materials during the electrochemical cycling is particularly important to the implementation of Li-ion batteries. This year, we studied the electronic structure of the $\mathrm{Li}$-ion deintercalated $\mathrm{Li}_{(1-\mathrm{x})} \mathrm{Co}_{1 / 3} \mathrm{Ni}_{1 / 3} \mathrm{Mn}_{1 / 3} \mathrm{O}_{2}$ materials with soft X-ray absorption spectroscopy (XAS) at $\mathrm{O} K$-edge and metal $\mathrm{L}_{\alpha, \beta}$, -edges, in combination with metal $K$-edge XAS spectra in the hard $\mathrm{x}$-ray region to elucidate the charge compensation mechanism. We found that a large portion of the charge compensation during Li-ion deintercalation is achieved in the oxygen site due to the presence of Co.

Characterization of the triple phase boundary in solid oxide fuel cells (SOFC), where the electron- and ion-conducting phases and the gas transport (void) phases meet, is a priority in understanding SOFC performance and durability. By combining studies of the large microstructure scale range, accessible using the NIST-built ultrasmall-angle $x$-ray scattering (USAXS) facility at the Advanced Photon Source, with anomalous small-angle x-ray scattering (ASAXS) measurements, it has been demonstrated that ASAXS can provide the differential contrast for distinguishing between the ion- and electron-conducting phase morphologies.

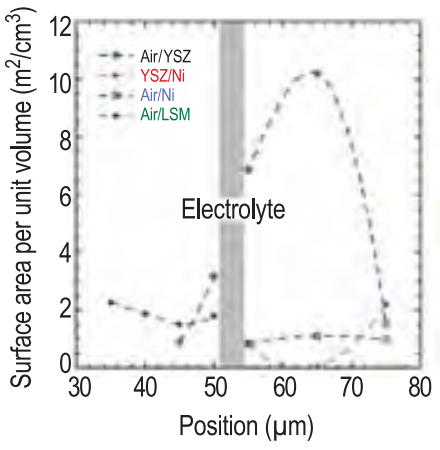

Figure 1: Spatial variation in component phase surface areas.

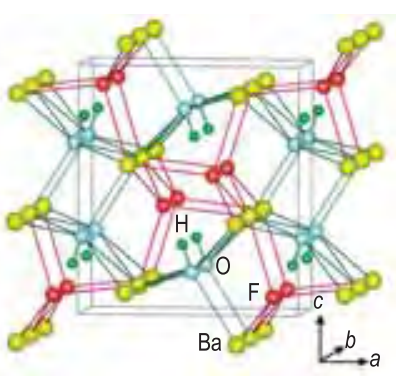

Figure 2: Structure of $\mathrm{Ba}(\mathrm{OH}) \mathrm{F}$.
Figure 1 shows the spatial variation of the void/solid surface areas involving the ion-conducting yttria-stabilized zirconia (YSZ) and lanthanum strontium manganate (LSM), and Ni that are the electron-conducting phases in the cathode and anode layers.

Phase equilibria data are critical for the coated conductor high $\mathrm{T}_{\mathrm{C}}$ materials for cable, generator, fly-wheel, and transformer applications. As an integral part of a DOE R\&D program, phase diagrams were developed for the $\mathrm{Ba}-\mathrm{R}-\mathrm{Cu}-\mathrm{O}(\mathrm{R}=\mathrm{Tm}$ and $\mathrm{Yb})$ systems. The " $\mathrm{BaF}_{2}$ " process is a promising method for producing long-length coated conductors. We have investigated the role of low-temperature melt and intermediate superlattice phases in the formation of $\mathrm{Ba}_{2} \mathrm{YCu}_{3} \mathrm{O}_{6+\mathrm{x}}(\mathrm{Y}-213)$. A new $\mathrm{Ba}(\mathrm{OH}) \mathrm{F}$ phase (Figure 2) was discovered that may be related to the low-temperature melting. The interaction of Y-213 with $\mathrm{SrTiO}_{3}$ substrates was studied in terms of phase equilibria of the Ba-Sr-Y-Cu-Ti-O system.

In collaboration with the University of South Florida, we have characterized the structure and provided $\mathrm{x}$-ray reference patterns for two clathrate phases ( $\mathrm{Sr}_{8} \mathrm{Ga}_{16} \mathrm{Ge}_{30}$ and $\mathrm{Cs}_{8} \mathrm{Na}_{16} \mathrm{Ge}_{136}$ ) that are promising candidates for thermoelectric power conversion applications. The structure of $\mathrm{Na}_{1-\mathrm{x}} \mathrm{Ge}_{3}$, which often coexists with $\mathrm{Cs}_{8} \mathrm{Na}_{16} \mathrm{Ge}_{136}$, was also studied. Defining the industrial needs for thermoelectric metrologies and the uses of combinatorial approaches for materials optimization remain a high priority.

\section{Contributors and Collaborators}

F. Biancaniello, M. Green, R. Radebaugh, G. Nolas (USF); Z. Yang, I. Levin, Q. Huang, R. Feenstra (ORNL); A. Goyal, V. Maroni (ANL); W.S. Yoon (BNL); K.Y. Chung, X.Q. Yang, J. McBreen, M. Balasubramanian, C.P. Grey, J. Ilavsky (APS XOR); P.R. Jemian (University of Illinois); J. Ruud (GE), W. Dawson (Nex Tech); A. Virkar (University of Utah) 


\section{Polymer Photoresists for Nanolithography}

Photolithography, the process used to fabricate integrated circuits, is the key enabler and driver for the microelectronics industry. As lithographic feature sizes decrease to the sub $65 \mathrm{~nm}$ length scale, challenges arise because both the image resolution and the thickness of the imaging layer approach the macromolecular dimensions characteristic of the polymers used in the photoresist film. Unique high-spatial resolution measurements are developed to reveal limits on materials and processes that challenge the development of photoresists for next-generation sub $65 \mathrm{~nm}$ lithography.

\section{Vivek M. Prabhu}

$\mathrm{P}$ hotolithography is the driving technology used by the microelectronics industry to fabricate integrated circuits with ever decreasing sizes. In addition, this fabrication technology is rapidly being adopted in emerging areas in optoelectronics and biotechnology requiring the rapid manufacture of nanoscale structures. In this process, a designed pattern is transferred to the silicon substrate by altering the solubility of areas of a polymer-based photoresist thin film through an acid-catalyzed deprotection reaction after exposure to radiation through a mask (Figure 1). To fabricate smaller features, next-generation photolithography will be processed with shorter wavelengths of light requiring photoresist films less than $100 \mathrm{~nm}$ thick and dimensional control to within $2 \mathrm{~nm}$.

To advance this key fabrication technology, we work closely with industrial collaborators to develop and apply high-spatial resolution and chemically specific measurements to understand changes in material properties, interfacial behavior, and process kinetics that can significantly affect the patterning process at nanometer scales.

This year, we initiated two new collaborations. With SEMATECH, we are determining the materials sources of line-edge roughness in model 193-nm photoresists. With the Intel Corporation, we are investigating the effect of extreme ultraviolet (EUV) exposure on pattern resolution of model EUV photoresist materials. With these partners, we continue to provide new insight and detail into the complex physico-chemical processes used in advanced chemically amplified photoresists. These methods include X-ray and neutron reflectivity (XR, NR), small angle neutron scattering (SANS), near-edge $\mathrm{x}$-ray absorption fine structure (NEXAFS) spectroscopy, combinatorial methods, solid state nuclear magnetic resonance (NMR), quartz crystal microbalance (QCM), Fourier transform infrared spectroscopy (FTIR), fluorescence correlation spectroscopy (FCS), and atomic force microscopy (AFM).

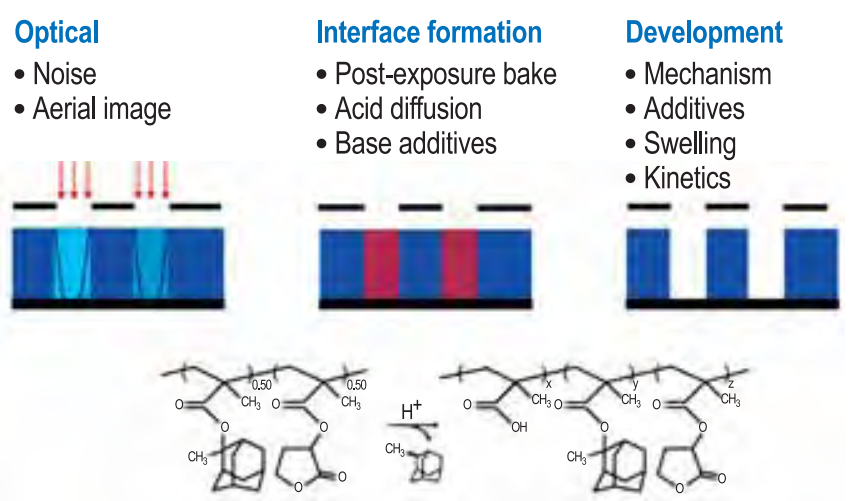

Figure 1: Key lithographic process steps; each step requires an interdisciplinary array of experimental techniques to measure the polymer chemistry and physics in thin films. A model 193-nm resist under investigation is shown with the acid-catalyzed deprotection reaction.

Photoresists are multi-component mixtures that require dispersion of additives, controlled transport properties during the interface formation, and controlled dissolution behavior. The fidelity of pattern formation relies on the materials characteristics. We examine the influence of copolymer compositions, molecular weight, and photoacid generator additive size to determine the root causes of image quality by highlighting the fundamental polymer physics and chemistry. In addition, our collaborators test our hypotheses using 193-nm and EUV lithographic production tools.

Accomplishments for this past year include: quantification of the developer profile in ultrathin films by NR and QCM; quantification of the deprotection reaction kinetics and photoacid-reaction diffusion deprotection front for resolution and roughness fundamentals by combined NR and FTIR; photoacid generator miscibility and dispersion in complex photoresist co- and ter-polymers by NMR; and aqueous immersion dependence on photoresist component leeching by NEXAFS.

\section{Contributors and Collaborators}

B. Vogt, A. Rao, S. Kang, D. VanderHart, W. Wu, E. Lin (Polymers Division, NIST); D. Fischer, S. Sambasivan (Ceramics Division, NIST); S. Satija (NIST Center for Neutron Research); K. Turnquest (Sematech); K-W. Choi (Intel); D. Goldfarb (IBM T.J. Watson Research Ctr); H. Ito, R. Allen (IBM Almaden Research Ctr); R. Dammel, F. Houlihan (AZ Electronics); J. Sounik, M. Sheehan (DuPont Elect. Polymers) 


\section{Organic Electronics}

Organic electronics has dramatically emerged in recent years as an increasingly important technology encompassing a wide array of devices and applications including embedded passive devices, flexible displays, and sensors. Device performance, stability, and function critically depend upon charge transport and material interaction at the interfaces of disparate materials. We develop and apply nondestructive measurement methods to characterize the electronic and interfacial structure of organic electronics materials with respect to processing methods, processing variables, and materials characteristics.

\section{Eric K. Lin and Dean M. DeLongchamp}

$\mathrm{O}^{\prime}$ rganic electronic devices are projected to revolutionize new types of integrated circuits through new applications that take advantage of low-cost, high-volume manufacturing, nontraditional substrates, and designed functionality. The current state of organic electronics is slowed by the concurrent development of multiple material platforms and processes and a lack of measurement standardization between laboratories. A critical need exists for new diagnostic probes, tools, and methods to address these technological challenges.

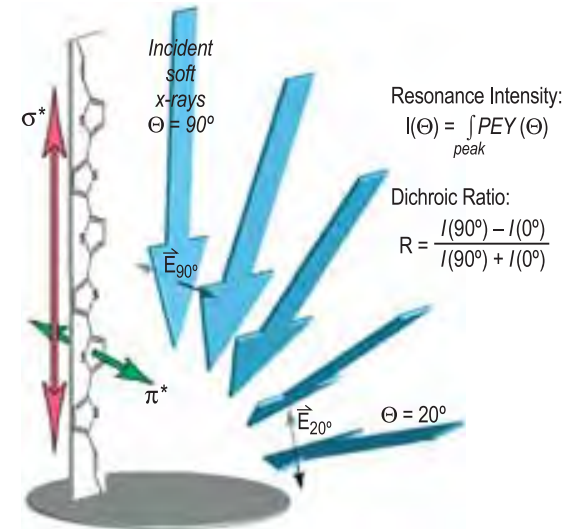

Figure 1: Schematic of the geometry of near-edge $x$-ray absorption fine structure (NEXAFS) spectroscopy for the determination of the orientation of an oligothiophene organic semiconductor synthesized by the University of California-Berkeley.

Organic electronics presents different measurement challenges from those identified for inorganic devices. We are developing an integrated suite of metrologies to correlate device performance with the structure, properties, and chemistry of materials and interfaces. We apply new measurement methods to provide the data and insight needed for the rational and directed development of emerging materials and processes. Studies include AC measurements of organic semiconductor thin films, the influence of surface modification layers on device performance, and the evaluation of moisture barrier layers for device encapsulation.

This year, near-edge $\mathrm{x}$-ray absorption fine structure (NEXAFS) spectroscopy was applied to several classes of organic electronics materials to investigate the electronic structure, chemistry, and orientation of these molecules near a supporting substrate. NEXAFS spectroscopy was used successfully to quantify the simultaneous chemical conversion, molecular ordering, and defect formation of soluble oligothiophene precursor films for application in organic field effect transistors. Variations in field-effect hole mobility with thermal processing were directly correlated to the orientation and distribution of molecules within $3 \mathrm{~nm}$ to $20 \mathrm{~nm}$ thick films.
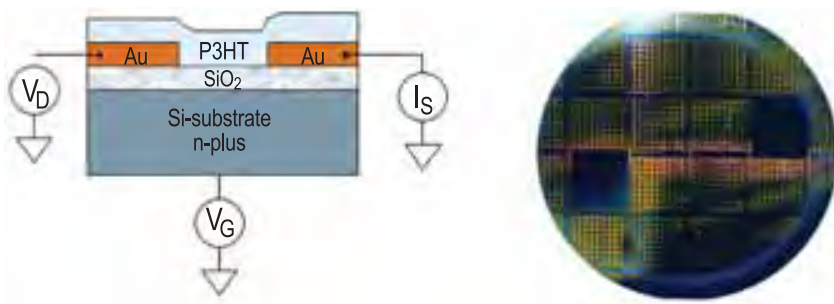

Figure 2: Schematic of an organic field effect transistor (OFET) and a photo of the NIST OFET test bed fabricated onto silicon.

Organic field effect transistor test structures were also designed and fabricated onto silicon wafers with variations in transistor channel length and width. Devices constructed using organic semiconductors such as poly(3-hexyl thiophene) (P3HT) were tested for their electrical characteristics such as the field effect hole mobility, on/off ratios, and threshold mobilities. Variations in mobility, for example, are observed with changes in processing variables such as annealing temperature and casting solvent. Correlations are found between device performance and the microstructure of P3HT as quantified by NEXAFS, optical ellipsometry, and FTIR spectroscopy.

\section{Contributors and Collaborators}

J. Obrzut, B. Vogel, C. Chiang, K. Kano, C. Brooks, N. Fisher, B. Vogt, H. Lee, Y. Jung, W. Wu (Polymers Division, NIST); S. Sambasivan, D. Fischer (Ceramics Division, NIST); M. Gurau, L. Richter (Chemical Science and Technology Laboratory, NIST); C. Richter, O. Kirillov (Electronics and Electrical Engineering Laboratory, NIST); R. Crosswell (Motorola); L. Moro, N. Rutherford (Vitex); A. Murphy, J.M.J. Frechet, P. Chang, V. Subramanian (University of California-Berkeley); M. Ling, Z. Bao (Stanford University); M. Chabinyc, Y. Wu, B. Ong (Xerox) 


\section{Nanoporous Low-k Dielectric Constant Thin Films}

\begin{abstract}
NIST provides the semiconductor industry with unique on-wafer measurements of the physical and structural properties of nanoporous thin films. Several complementary experimental techniques are used to measure the pore and matrix morphology of candidate materials. The data are used by industry to select candidate low-k materials. Measurement methods such as $x$-ray porosimetry and small angle $x$-ray scattering are developed that may be transferred to industrial laboratories. Methods are being developed to measure patterned low-k samples and to assess the extent of structure modification caused by plasma etch.
\end{abstract}

\section{Eric K. Lin and Wen-li Wu}

Tuture generations of integrated circuits will require 1 porous low-k interlayer dielectric materials to address issues with power consumption, signal propagation delays, and crosstalk that decrease device performance. The introduction of nanometer scale pores into a solid film lowers its effective dielectric constant. However, increasing porosity adversely affects other important quantities such as the physical strength needed to survive chemical mechanical polishing steps and barrier properties to contaminants such as water. These effects pose severe challenges to the integration of porous dielectrics into the device structure.

There is a need for nondestructive, on-wafer characterization of nanoporous thin films. Parameters such as the pore size distribution, wall density, porosity, film uniformity, elemental composition, coefficient of thermal expansion, and film density are needed to evaluate candidate low-k materials. NIST continues to develop low-k characterization methods using a combination of complementary measurement methods including small angle neutron and $\mathrm{x}$-ray scattering (SANS, SAXS), high-resolution x-ray reflectivity (XR), $\mathrm{X}$-ray porosimetry (XRP), SANS porosimetry, and ion scattering. To facilitate the transfer of measurement expertise, a recommended practice guide for XRP is available for interested researchers.

In collaboration with industrial and university partners, we have applied existing methods to new low-k materials and developed new methods to address upcoming integration challenges. A materials database developed in collaboration with SEMATECH is used extensively by SEMATECH and its member companies to help select candidate materials and to optimize integration processing conditions. We address the effects of the ashing/plasma etch process on the low- $\mathrm{k}$ material during pattern transfer. Often surfaces exposed to ashing/plasma densify and lose terminal groups (hydrogen or organic moiety) resulting in an increased moisture adsorption and thus dielectric constant. XR measurements enable quantification of the surface densification or pore collapse in ashing-treated and/or plasma-treated blanket films.
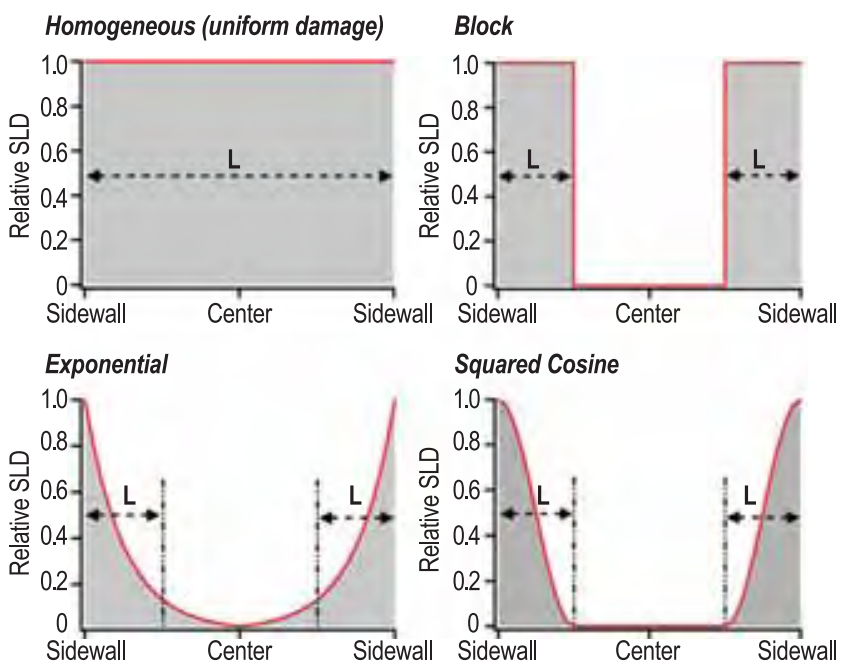

Figure 1: Four models of the damage layer profile plotted as scattering length density (SLD) as a function of position within a line. The matrix has a relative SLD of zero in the above plots.

This year, a new method using SAXS was developed to investigate the effect of plasma etch on patterned low-k films. After the plasma etch process, samples are backfilled with the initial low-k material. Any densification of the sidewall may be observable by $\mathrm{x}$-ray scattering from the cross-section of a patterned nanostructure. This SAXS work was carried out at Argonne National Laboratory using line gratings of low-k material. The resulting data can then be compared with several different scattering models for the densification of the patterned low-k material as shown in Figure 1. Distinctions between models such as these will significantly help semiconductor manufacturers to accelerate the integration of low-k materials into next generation devices.

\section{Contributors and Collaborators}

H. Lee, C. Soles, R. Jones, H. Ro, D. Liu, B. Vogt (Polymers Division, NIST); C. Glinka (NIST Center for Neutron Research); Y. Liu (SEMATECH); Q. Lin, A. Grill, H. Kim (IBM); J. Quintana, D. Casa (Argonne National Laboratory); K. Char, D. Yoon (Seoul National University); J. Watkins (University of Massachusetts) 


\section{Electrical Methods for Mechanical Testing}

The International Technology Roadmap for Semiconductors calls for solutions to the near-term challenge (through 2009) of achieving necessary reliability because "new materials, structures, and processes create new chip reliability (electrical, thermal, and mechanical) exposure. Detecting, testing, modeling and control of failure mechanisms will be key." To this end, we develop test and detection methodologies for mechanical reliability of dimensionally-constrained materials, and further materials science understanding of the observed mechanical behavior.

\section{Robert R. Keller, Nicholas Barbosa III, Roy H. Geiss, David T. Read, and Andrew J. Slifka}

$\mathrm{T}$ $\neg$ his year we have consolidated three distinct 1 projects addressing different aspects of the reliability of materials for microelectronics into one Division program. The present focus is on electrical methods for measuring thermal fatigue lifetime and mechanical strength of patterned metal films. These methods are based on the principle of applying Joule heating to specimens in a controlled manner, by use of low-frequency, high-current density a.c. electrical signals. Thermal expansion mismatch between film and substrate then leads to thermal strains, which can be used as the basis for mechanical testing.

A key aspect of developing time-varying electrical methods for mechanical metrology is knowledge of the temperature of the specimen, with sufficient spatial and temporal resolutions. Details are provided in the Materials Reliability Division Technical Highlights section.

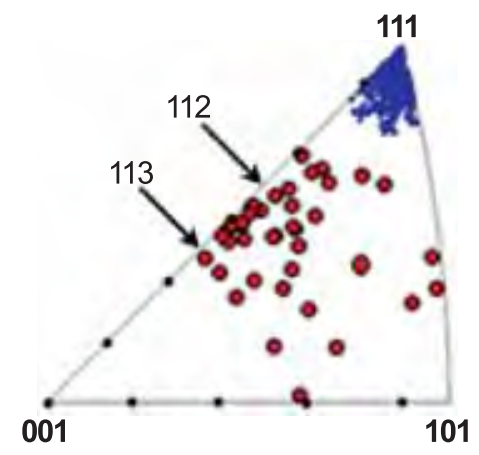

Figure 1: Inverse pole figure showing surface normal orientations before and after a. c. stressing of Al-1Si at $12.2 \mathrm{MA} / \mathrm{cm}^{2}$ for $1.4 \times 10^{5}$ temperature cycles.

We have made additional progress in documenting the damage processes leading to failure during thermal fatigue of patterned interconnects, using $\mathrm{Al}-1 \mathrm{Si}$ as a model material. Figure 1 depicts an inverse pole figure (IPF) showing surface normal grain orientations before and after a.c. stressing. Blue dots represent orientations prior to stressing, and red dots represent orientations of severely deformed regions after stressing. Black dots along IPF edges indicate $10^{\circ}$ increments. Reorientation is consistent with a recent analysis of slip asymmetry in cyclic deformation of bulk fcc crystals, which showed that for the case of fully reversed loading, a single crystal reorients such that during the tensile half-cycle, the loading axis rotates toward the primary slip direction, [011], and during the compressive half-cycle, it rotates toward the normal to the primary slip plane, $(1-11)$. The net result of this ratcheting action is an ultimate crystal orientation near (113).

Development of methods to electrically measure strengths of thin films is now underway, with microtensile testing as a reference throughout. Yield strength measurement is based on detection of the onset of plasticity using changes in residual resistance after accumulated cyclic damage. Ultimate strength measurement is based on extrapolation of cyclic data to a single cycle, by use of the Coffin-Manson rule.

Results from our work appeared this year in two archival journal articles, and we target three more journal submissions by October 2005; this series of papers will establish us as the leaders in this novel approach to mechanical testing of materials with constrained dimensions. One paper written with German colleagues (R. Mönig, R.R. Keller, C.A. Volkert, "Thermal Fatigue Testing of Thin Metal Films,” Rev. Sci. Instrum. 75, 4997-5004 (2004)) represents the first archival journal publication of this work and describes in detail the test methodology. We also completed the final report of our August 2004 workshop on Reliability Issues in Nanomaterials (R.R. Keller, D.T. Read, and R. Mahajan, Report of the Workshop on Reliability Issues in Nanomaterials, NIST-SP, in BERB), which was reviewed by nine non-NIST plenary speakers; this workshop established us as being among the recognized leaders in the broad field of reliability of nanomaterials.

Seven presentations of this work were given at five conferences in FY05 (MRS, ASME, Characterization and Metrology for ULSI Technology, GOMACTech, Mechanics and Materials); two more conference presentations are planned in October 2005.

\section{Contributors and Collaborators}

Y. Cheng (Protiro, Inc.); R. Mönig (MIT); C. Volkert (Forschungszentrum, Karlsruhe, Germany); B. Sun (Intel) 


\section{Thermochemical Metrology of Interfacial Stabilities}

Hetero-interfaces are present in the majority of electronic materials and affect the operating characteristics of many devices. For successful fabrication and optimal performance of advanced designs, data on the behavior of interfaces during thermal processing is essential. Efficient measurement and prediction of the interfacial thermochemical stabilities of potentially useful materials combinations require new technology and new approaches, which are being developed in our laboratories.

\section{Lawrence P. Cook, Mark Vaudin, and Martin L. Green}

$\mathrm{B}$ y using thin-film differential scanning calorimeters (DSCs), it is possible to study materials interactions on a very fine scale - both in terms of the mass of the samples involved, as well as the magnitude of the thermodynamic quantities measured. A further advantage of the thin-film DSC approach to thermochemical metrology is that it lends itself to combinatorial studies, in which compositionally graded thin films can be deposited over a DSC array. In this way, interfacial stabilities of various materials combinations can be rapidly evaluated. Our plan is to correlate thermochemical data from DSC arrays with characterization by $\mathrm{x}$-ray microdiffraction, using an area detector and an automated stage to sample each element of the array. The products formed during interfacial reaction will be identified, and these experiments will form the basis for more detailed examination of the kinetics of reaction for appropriate materials combinations.

To date, our efforts on thin-film DSC have concentrated on proof-of-concept using well-known

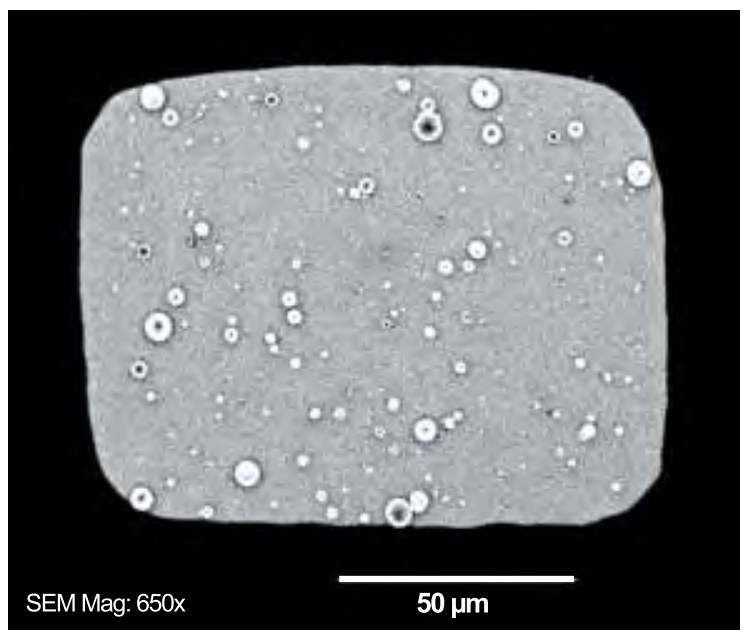

Figure 1: PLD-deposited Sn on DSC sensor.

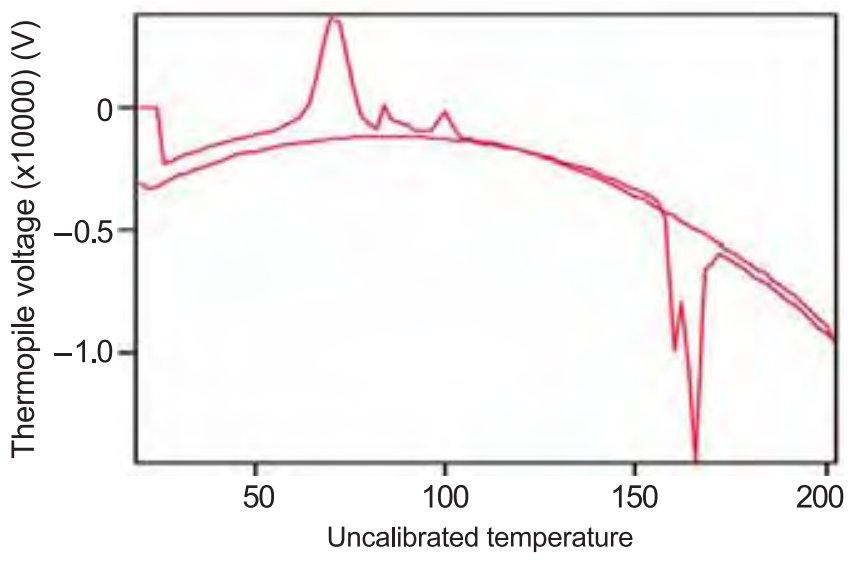

Figure 2: Thin-film DSC of Sn in Figure 1.

thermochemical events such as the melting of Sn $\left(232{ }^{\circ} \mathrm{C}\right)$, the $\varepsilon / \alpha$ transition in metallic Co $\left(422{ }^{\circ} \mathrm{C}\right)$, and the reaction between elemental $\mathrm{Si}$ and $\mathrm{Ni}$ thin films (kinetically determined). Studies of the latter two reactions are still in progress, but our preliminary studies of $\mathrm{Sn}$ melting have been completed. Figure 1 shows a $200 \mathrm{~nm}$ thick layer of Sn deposited on the sample side of a thin-film DSC sensor. The sample was deposited by pulsed laser deposition (PLD) through a $60 \mu \mathrm{m}$ x $100 \mu \mathrm{m}$ mask; a range of particle sizes is evident. Figure 2 is an uncalibrated average of multiple DSC scans from a sample like that in Figure 1. The melting (endothermic) and crystallization (exothermic) scans show multiple events, possibly related to differences in thermal behavior of different size fractions of the particles. A large thermal hysteresis between melting and crystallization is evident. This example suggests that the thin-film DSC is sensitive to variations in materials properties, in this case probably influenced by the interfacial tension between the metallic $\mathrm{Sn}$ in the core of the particles and the thin $\mathrm{SnO}_{2}$ skin on the surface of the particles.

Work has already begun on a second generation of thin-film DSCs that will be optimized to give true nanometric sensitivity. This work is being done in collaboration with the University of Illinois at Urbana-Champaign. Currently, instrumentation is being set up to utilize the enhanced sensitivity that will be achieved through a chip design minimizing thermal mass. With the newer devices, near-adiabatic measurements will be possible.

\section{Contributors and Collaborators}

R. Cavicchi, C. Montgomery, S. Semancik, M. Carrier (Process Measurements Division, NIST); P. Schenck, W. Wong-Ng, J. Blendell (Ceramics Division, NIST); L. Allen (University of Illinois, UrbanaChampaign); M. Efremov (University of Wisconsin) 


\section{Combinatorial Metal Selection for Catalytic Growth of $\mathrm{ZnO}$ Semiconductor Nanowires}

\begin{abstract}
Semiconductor nanowires (NWs) offer a unique type of nanoscale building block for creating next-generation lasers and chemical/biological sensors. Control of NW dimension and structural and electronic properties is a major barrier to device fabrication. To address these issues, we are developing a strategy for selecting metals for catalytic growth of zinc-oxide ( $\mathrm{ZnO})$ nanowires using an approach based on phase diagrams and high-throughput fabrication and analysis.
\end{abstract}

\section{Albert V. Davydov}

Semiconductor NWs show significant promise for a wide variety of optoelectronic and electronic devices including nano-lasers, detectors, and chemical and biological sensors. To advance commercialization of such devices, it is necessary to control properties including size, orientation, and structural and electronic defects of the fabricated nanostructures.

NWs are often produced using a vapor-liquid-solid (VLS) approach, in which nanometer size islands of catalytic metals act as nucleation and growth sites for semiconductor NW growth. The choice of the metal catalyst can significantly affect the NW properties. We are developing a strategy for selecting appropriate catalytic metals and applying this strategy to VLS growth of ZnO semiconductor. The selection and screening of catalysts

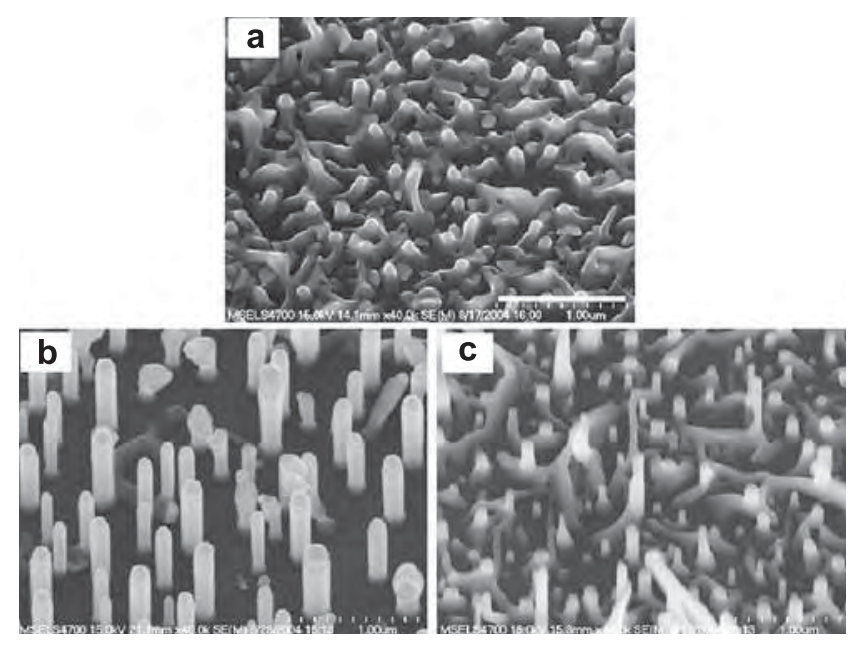

Figure 1: Scanning electron microscope images from the combinatorial library: (a) $\mathrm{ZnO}$ wetting layer grown using gold catalyst (note the absence of NWs); (b) ZnO NWs grown using gold-copper alloy catalyst; (c) $\mathrm{ZnO} N$ Ws grown using silver catalyst. Growth temperature $850^{\circ} \mathrm{C}$. The scale bar on the top image is $1 \mu \mathrm{m}$. has been achieved using thermodynamic information from phase diagrams as well as a high-throughput (combinatorial) approach recently demonstrated for metallizations to wide-band-gap semiconductors. ${ }^{[1]}$

We have examined the role of elemental noble metals and their alloys on ZnO NW growth and properties. An experimental library was designed using the ternary silver-gold-copper phase diagram and included elemental, binary and ternary compositions as well as two growth temperatures (850 and $950{ }^{\circ} \mathrm{C}$ ). The metal library elements were deposited on a single gallium nitride/sapphire substrate, which was subsequently annealed to form isolated metal droplets upon which the $\mathrm{ZnO}$ NWs were grown. The resulting set of metal islands and $\mathrm{ZnO}$ NWs was characterized using electron microscopy, x-ray diffraction, and optical spectroscopy. It was found that the ZnO NW growth was significantly influenced by the nature of the catalytic metal droplets formed on the substrate. For example, growth from the $\mathrm{Au}$ islands at $850{ }^{\circ} \mathrm{C}$ yielded only a thin $\mathrm{ZnO}$ nucleation layer with no NWs, while other metal compositions, which melt at lower temperatures than $\mathrm{Au}$, produced a variety of NW shapes, sizes and orientations at this temperature (Figure 1).

A combinatorial approach also permits rapid correlation of the optical properties of the nanowires with the composition of the metal islands from which they were grown. For example, $\mathrm{ZnO}$ NWs fabricated from the silver islands at $850^{\circ} \mathrm{C}$ produced the sharpest excitonic peak near $3.36 \mathrm{eV}$ on the cathodoluminescence spectrum (comparable to that for bulk $\mathrm{ZnO}$ single crystal), indicating superior crystalline structure. In contrast, the spectra were significantly broader for the NWs grown from the gold and copper containing islands. A comprehensive assessment of the correlation of structural and spectroscopic data for the ZnO NW library is still underway. These initial results demonstrate the power of the combined phase diagram/combinatorial approach for controlling semiconductor NW properties.

1. A.V. Davydov, A. Motayed, W.J. Boettinger, R.S. Gates, Q.Z. Xue, H.C. Lee, and Y.K. Yoo, Phys. Stat. Sol.(c), 2(7), 2551.

\section{Contributors and Collaborators}

W. Boettinger, D. Josell, C. Handwerker, U. Kattner, M. Vaudin, I. Levin, L. Robins (MSEL); B. Nikoobakht (CSTL); N. Sanford (EEEL); A. Motayed (University of Maryland) 


\section{Advanced Processes and Materials for On-Chip Interconnects}

As dimensions of copper wiring in on-chip electrical conductors drop below $100 \mathrm{~nm}$, control of electrical resistivity has become critical. We have demonstrated new barriers for seedless deposition processes that will simplify processing and replace high resistivity tantalum barriers. We have also demonstrated gold superfill as an alternative metallization.

\section{Daniel Josell and Thomas P. Moffat}

$\Delta$ s dimensions of transistors in integrated circuits 1 shrink, the dimensions of the metal wires connecting them shrink as well. With industry passing through the $90 \mathrm{~nm}$ node, electron transport is reduced and fabrication is increasingly difficult.

\section{A.}
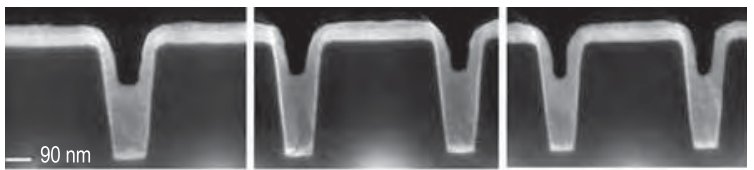

B.
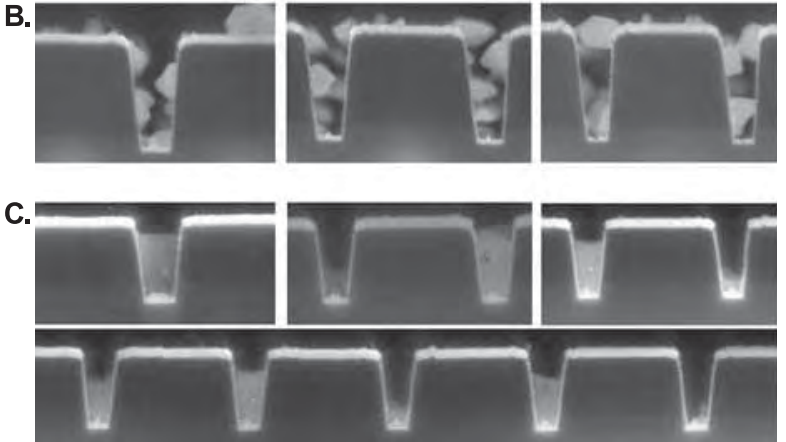

Figure 1: Copper superfill in trenches with ruthenium barriers and no copper seed. (A) Desired copper wetting and superfill. (B) Poor wetting in the presence of surface oxide. (C) Intriguing localized deposition in trenches, the origin of which is unknown.

Consistent with dimensional requirements specified in the International Technology Roadmap for Semiconductors, we have been exploring new barrier materials that will permit copper electrodeposition without a copper seed layer. We have explored platinum group elements and have found several that permit seedless deposition. As part of this effort, we have explored in depth the role of oxide removal and underpotential deposition of copper in determining the wettability of ruthenium barriers and associated reliability for seedless copper superfill. This work has demonstrated a process for "repair" of seeds through removal of surface oxide.

We have also explored novel metallizations.

With the successful demonstration of gold superfill this year (Figure 2), we have now established processes for superfill with three metals, our work being the first to demonstrate superfill with either silver or gold.
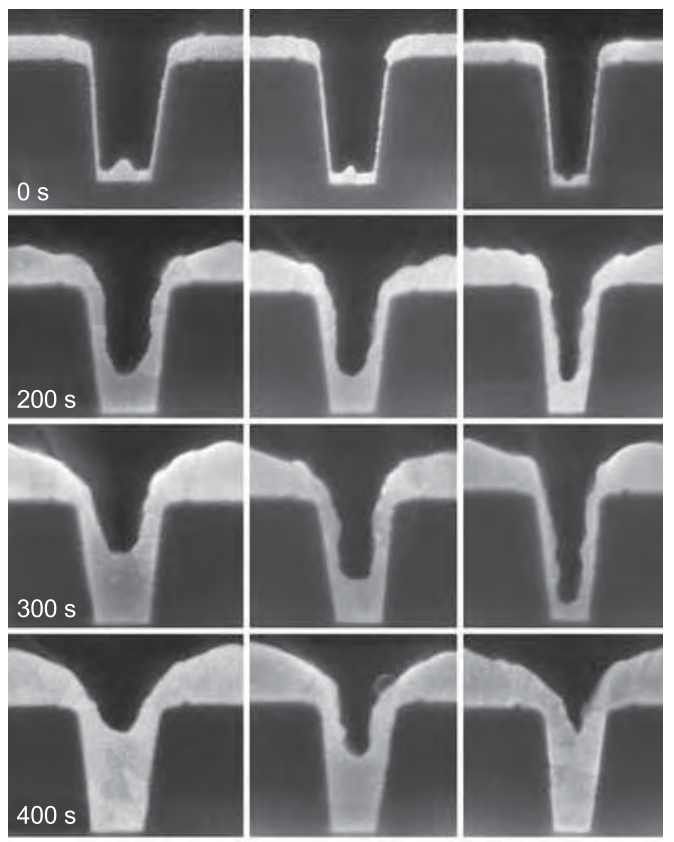

$300 \mathrm{~nm}$

Figure 2: Gold superfill in trenches of three different aspect ratios after indicated deposition times, showing bottom-up superfill.

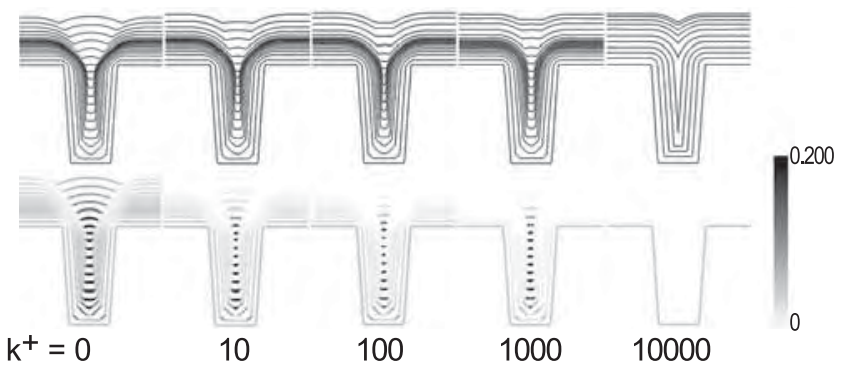

Figure 3: The impact of leveling additives on trench superfill and overfill bump formation. Higher adsorption kinetics $k^{+}$ leads to reduced overfill bump formation (top), consistent with experimental observation. Corresponding coverage of accelerator is shown beneath.

Recognizing the significance of leveling additives in industrial processing, we have now extended our Curvature Enhanced Accelerator Coverage (CEAC) mechanism to account for their presence during superfill. The resulting equations predict reduction of overfill bump through the use of levelers (Figure 3), consistent with observation. Experiments are underway to assess kinetics to permit quantitative comparison to experiment.

\section{Contributors and Collaborators}

C. Witt (Cookson-Enthone); D. Wheeler (University of Maryland) 


\section{Pb-free Surface Finishes: Sn Whisker Growth}

Soldered joints are increasingly tenuous links in the assembly of microelectronics as a consequence of ever shrinking chip and package dimensions, the broadening use of flip-chip technology, and the movement toward environmentally friendly lead-free (Pb-free) solders. To support needs in this area, the goal of this project is to provide data and materials measurements of critical importance to solder interconnect technology for microelectronics assembly.

\section{Maureen E. Williams and Kil-Won Moon}

T is well known that the use of pure tin protective 1 deposits can lead to problems with whiskers filamentary whiskers typically $1 \mu \mathrm{m}$ diameter and several mm long which grow from the plating and cause electrical shorts and failure. Historically, lead was added to tin plate to prevent whisker growth as well as lower costs. The present project focuses on $\mathrm{Pb}$-free, Sn-rich deposits with alloying additions that could potentially retard whisker formation. The Sn-Cu system was selected for compatibility reasons, since $\mathrm{Sn}-\mathrm{Cu}-\mathrm{Ag}$ is likely to be the $\mathrm{Pb}$-free solder of choice for industrial applications. The substitution of a different solute for $\mathrm{Pb}$ in the Sn-rich deposit was proposed to also retard whisker growth. A detailed microstructural comparison of deposits with high- and low-whiskering tendency has been conducted, leading to a correlation of Sn grain size, shape, and residual stress with whisker growth.

A manuscript submitted to Acta Materialia, "Hillock and Whisker Formation in Sn, Sn-Cu and $\mathrm{Sn}-\mathrm{Pb}$ Electrodeposits," presents measurements of compressive stress in the electrodeposits and how various microstructural features of the deposits affect whisker growth. Relief of the compressive stress occurs by uniform creep for $\mathrm{Sn}-\mathrm{Pb}$ because it has an equiaxed grain structure. On the other hand, localized creep in the form of hillocks and whiskers occurs for $\mathrm{Sn}$ and $\mathrm{Sn}-\mathrm{Cu}$ because both have columnar grain structures. Compact hillocks form for Sn deposits because the columnar grain boundaries are mobile. Contorted hillocks and whiskers form for the $\mathrm{Sn}-\mathrm{Cu}$ deposits because the columnar grain boundary motion is pinned by intermetallic precipitates.

Some reports suggest that restraining and cracking of a tin oxide surface film are necessary steps in the nucleation of Sn whiskers. However, results at NIST, accepted for publication in the Journal of Electronic Materials showed little support for this mechanism.

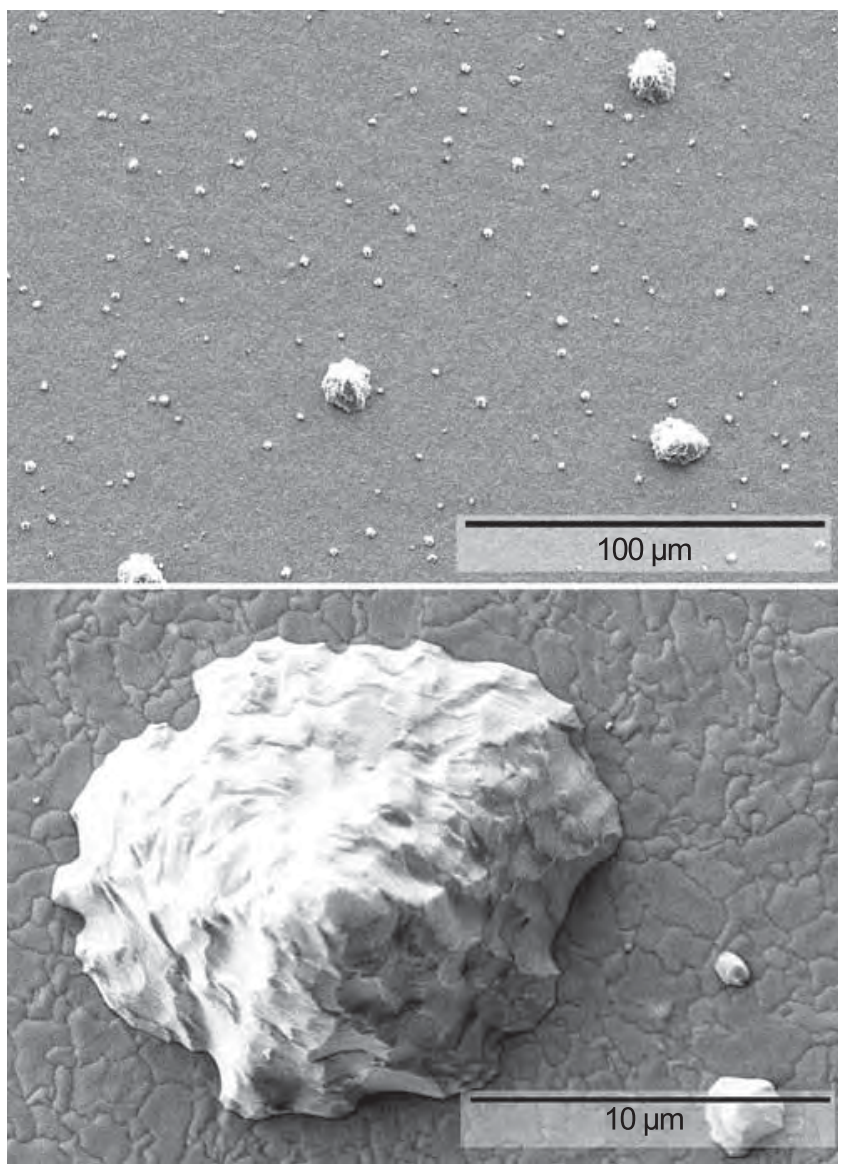

Figure 1: Low and high magnification SEM micrographs of the electrodeposited surface of a $16 \mu \mathrm{m}$ thick pure Sn layer on a cantilever beam showing bimodal size distribution of conical hillocks. Such hillocks form to relieve compressive residual stress. Whiskers form when grain boundaries in the deposit are pinned by precipitates.

For instance, whiskers were observed on bright $\mathrm{Sn}-\mathrm{Cu}$ electrodeposits but not on pure bright Sn electrodeposits on pyrophosphate $\mathrm{Cu}$ substrates. In order to understand the role of the deposit surface, the Sn oxide surface film and surface structures were analyzed by Auger and Electron Backscatter Diffraction (EBSD). In Auger analysis, residuals of the Sn oxide surface film were observed after $\mathrm{Ar}+$ ion cleaning. This feature allowed us to discriminate the Sn whisker growth with or without the oxide surface film.

\section{Contributors and Collaborators} NIST) 


\section{Materials for Advanced Si CMOS}

Further size reduction of complementarymetal-oxide-silicon (CMOS) devices in the Si microelectronics industry, necessary for continued adherence to Moore's law, is currently materials limited. The gate stack, composed of the $\mathrm{SiO}_{2}$ gate dielectric and the doped polycrystalline Si gate electrode, is no longer sufficient and must be replaced by new materials. Further, starting Si wafers must be strain engineered to increase carrier mobility and subsequent device performance. NIST plays an active role in both activities.

\section{Martin L. Green}

$\mathrm{N}$ IST/MSEL is poised to play important roles in the introduction of new materials to the Si microelectronics industry, so that further scaling (size reduction) may be enabled. One example is the advanced gate stack for Si CMOS. To enable further device scaling, the capacitive equivalent thickness (CET) of the gate stack must be $0.5 \mathrm{~nm}$ to $1.0 \mathrm{~nm}$. This will not be achievable with existing $\mathrm{SiO}_{2}$ /polycrystalline $\mathrm{Si}$ gate stacks. Since replacements for $\mathrm{SiO}_{2}$ have already been identified, it is now particularly important to replace the doped polycrystalline Si gate electrode with a true metal. Given the large number of possible choices of alloys for the metal gate electrode, the combinatorial approach is seen as the most effective way of identifying alloys possessing the proper work function and stability as metal gates on $\mathrm{HfO}_{2}$.

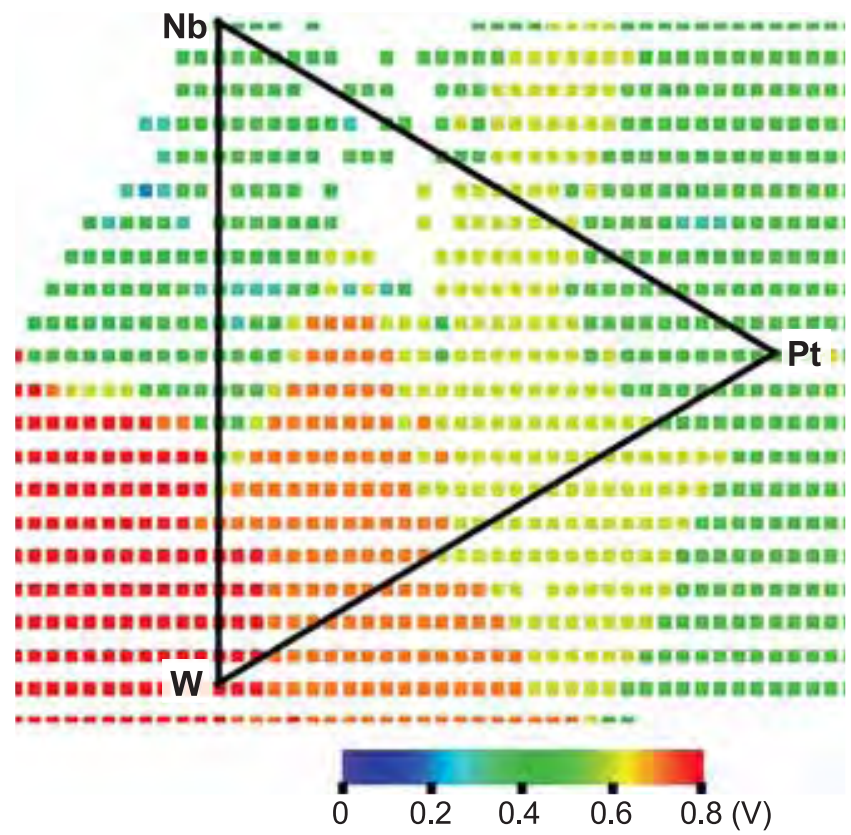

Figure 1: Flatband voltage measurements obtained from capacitance - voltage data, for the $\mathrm{Nb}-\mathrm{Pt}-\mathrm{W}$ metal gate system.

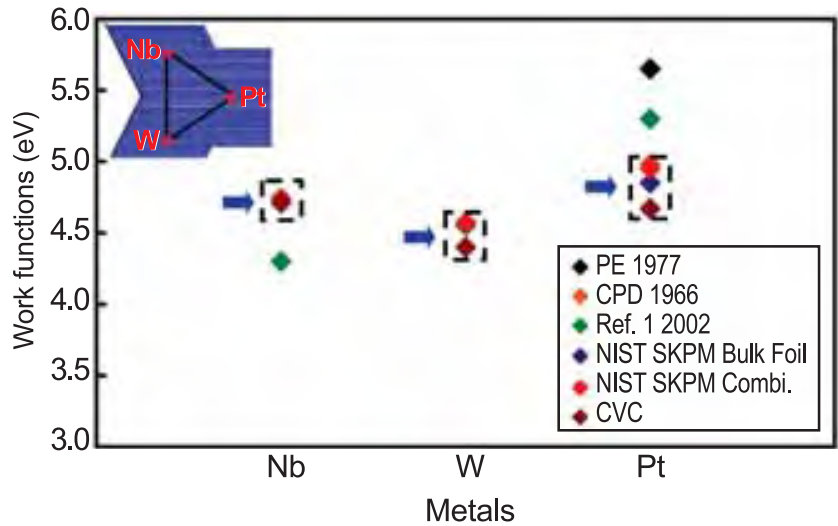

Figure 2: Comparison of work function data determined through combinatorial experiments and other techniques.

Figure 1 shows flatband voltage data, from which work functions may be derived, measured on hundreds of capacitors, where each small square represents a different metal gate composition in the Nb-W-Pt ternary system. Variations in flatband voltage, due to alloy composition, are readily observed. As can be seen from Figure 2, work functions determined through combinatorial experiments are in excellent agreement with those determined by other, less straightforward means.

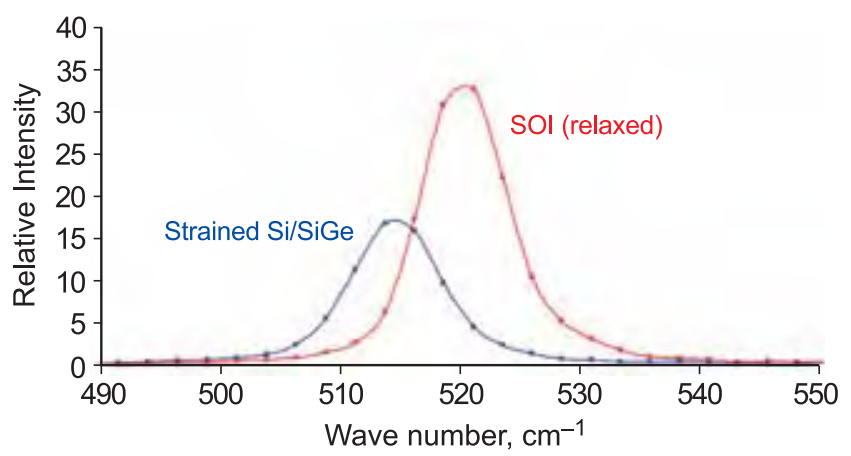

Figure 3: Raman shift of strained Si with respect to relaxed Si.

Another example of NIST activity in advanced Si materials is strained $\mathrm{Si}$, which is increasingly being used to enhance carrier mobilities in high performance devices. Figure 3 shows the characteristic Raman shift that accompanies strain in the Si lattice. MSEL/NIST is working to calibrate the Raman shift to an absolute strain measurement (via precision lattice parameter determination) to facilitate the introduction of this technology.

\section{Contributors and Collaborators}

D. Black, K.-S. Chang, T. Chikyow (NIMS, Japan); M. Gardener (Sematech); D. Josell, R. Lei (Intel); P. Majhi (Sematech); A. Paul, P. Schenck, W. Wong-Ng, J. Suehle (EEEL/NIST); I. Takeuchi (University of Maryland) 


\section{Metrology and Standards for Electronic and Optoelectronic Materials}

The electronics and optoelectronics industries require accurate materials properties data to improve their device fabrication, modeling, and evaluation processes. Our recent activities have emphasized optical and structural metrologies applicable to wide bandgap semiconductor nanowires, and UV Raman spectroscopy for the evaluation of strain in strained silicon-on-insulator structures.

\section{Lawrence H. Robins and Albert J. Paul}

$\mathrm{N}$ anowires, defined as semiconductor or metal structures having a quasi-cylindrical geometry with diameter in the range 1 to $100 \mathrm{~nm}$, are expected to have a major impact on future electronic and optoelectronic technologies. A NIST program was recently started to develop growth and manipulation techniques, metrologies, and test structures for semiconductor nanowires, based on GaN growth by molecular beam epitaxy (MBE), and ZnO growth by the catalyst-assisted vapor-liquid-solid (VLS) method. We are contributing to this program by developing metrologies for structural and optical characterization of the nanowire samples and test structures.
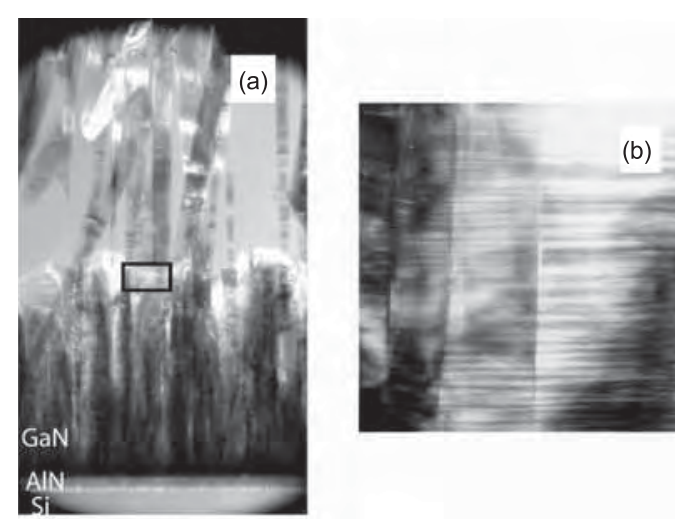

Figure 1: Dark-field TEM images of GaN nanowire and matrix structure grown on AlN/Si. (a) Lower magnification image shows nanowires and unwanted matrix layer both growing from AlN buffer. (b) Higher magnification image shows basal-plane defects in matrix layer.

TEM structure of one of our first GaN nanowire samples, grown by MBE on an AlN buffer layer on $\mathrm{Si}(111)$, is shown in Figure 1. The sample contains both nanowires and a rough, faceted "matrix" layer (lower half of Figure 1(a)). Growth of most nanowires appears to initiate at the AlN buffer, rather than within the matrix layer. The matrix layer contains a high density of basal-plane defects, which produce striations in the dark-field image (Figure 1(b)), and also give rise to streaking in electron diffraction (not shown here). In contrast, the nanowires appear free of extended defects in TEM.

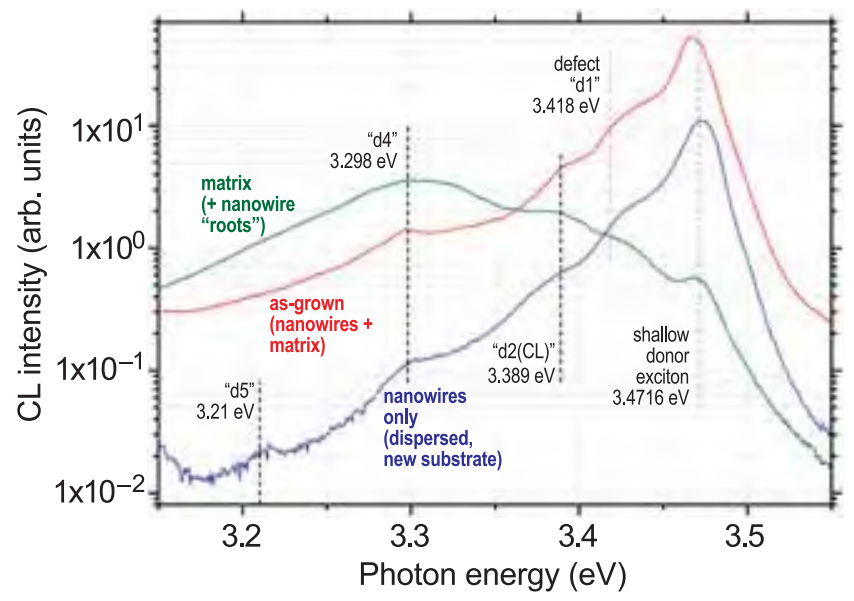

Figure 2: Low-temperature CL of GaN nanowire sample. Red curve: as-grown. Blue curve: nanowires only, removed from matrix layer. Green curve: matrix layer + nanowire "roots," with tops of nanowires polished off.

The nanowire structure from Figure 1 was characterized by low-temperature cathodoluminescence (CL), as shown in Figure 2. The CL of the nanowires is dominated by excitons bound to shallow donor impurities (3.4716 eV peak) while the matrix layer CL is dominated by excitons bound to structural defects (peaks "d1" through "d5") that may be related to the defects seen by TEM.

Similarly to the $\mathrm{GaN}$ samples, the $\mathrm{ZnO}$ samples were found to contain a highly defective "matrix" layer together with relatively defect-free vertical nanowires, but the matrix layer in the $\mathrm{ZnO}$ was found to consist of horizontally growing nanowires. The choice of metal catalyst (Ag, Au, or Cu-containing alloy) for VLS growth of the $\mathrm{ZnO}$ samples was found to have a strong affect on the CL spectra, ascribed to catalyst doping of the $\mathrm{ZnO}$ and concomitant formation of catalyst-related impurity levels. Our characterization results on $\mathrm{GaN}$ and $\mathrm{ZnO}$ nanowires were reported at the 2005 Electronic Materials Conference (Santa Barbara, CA, June 22-24).

\section{Contributors and Collaborators}

A. Davydov, A. Motayed (Metallurgy Division, NIST); J. Barker, K. Bertness, N. Sanford (Optoelectronics Division, NIST); B. Nikoobakht (Surface and Microanalysis Science Division, NIST); R.Z. Lei (Intel Corporation); G. Celler, M. Kennard (SOITEC) 


\section{Nano-Structured Materials for Sensors and Ultra-High Density Data Storage}

\author{
Magnetic sensors play a central role in many \\ important technologies ranging from health care \\ to homeland security. A common need among \\ these technologies is greater sensitivity through \\ nano-structured materials.
}

In ultra-high density data storage, one of the most pressing needs is for nano-structured media that store data at ever-increasing densities. Improved methods for the magnetic isolation of grains in ultra-thin films are a key need. We have initiated research programs in both areas.

\section{William F. Egelhoff, Jr.}

$\mathrm{O}$ ver the past decade, NIST's Magnetic Engineering Research Facility (MIRF) has made important and widely recognized contributions to the thin magnetic films used as read heads for hard disk drives. MIRF is one of the most versatile facilities in the world for the fabrication and analysis of novel magnetic thin films. This versatility is illustrated by two new areas of research that have recently been initiated. One is magnetic sensors and the other is magnetic media. The common link is that both require nano-structured thin films.

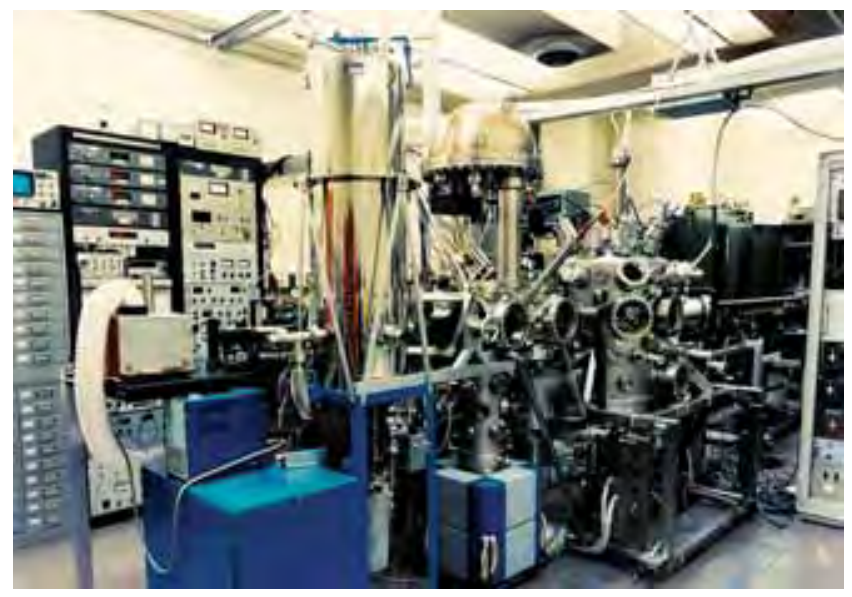

Figure 1: The Magnetic Engineering Research Facility.

We are now 1.5 years into the Magnetic Sensors Competence Program. We have evaluated a series of complex magnetic alloys that in bulk form are very sensitive to small magnetic fields but had not previously been studied in thin-film form (e.g., $\mathrm{Ni}_{77} \mathrm{Fe}_{14} \mathrm{Mn}_{5} \mathrm{Cu}_{4}$ ). Our approach is to carry out the metrology needed to optimize these materials in thin-film form.

We have made several surprising discoveries. First, these alloys are uniformly less sensitive in thin-film form. Second, some of the bulk sensitivity can be recovered by nano-layering the magnetic thin films with non-magnetic thin films. Third, conventional thin-films sensor designs contain a design flaw that allows magnetic irregularities to reduce the potential sensitivity by over a factor of 100 . Fourth, we have found a way to use nano-structuring of the thin films to reduce these irregularities sharply and achieve a factor of 40 improvement in sensitivity. Fifth, an analysis of the physics of the nano-structuring immediately suggests that opportunities exist for significant further gains.

In the area of novel magnetic media we have been collaborating very closely with Seagate. Seagate is the world's leading manufacturer of hard-disk drives, and for the past two years, they have been sending a Ph.D. physicist from their research labs to work with us, first on ballistic magnetoresistance, and then on novel magnetic media.

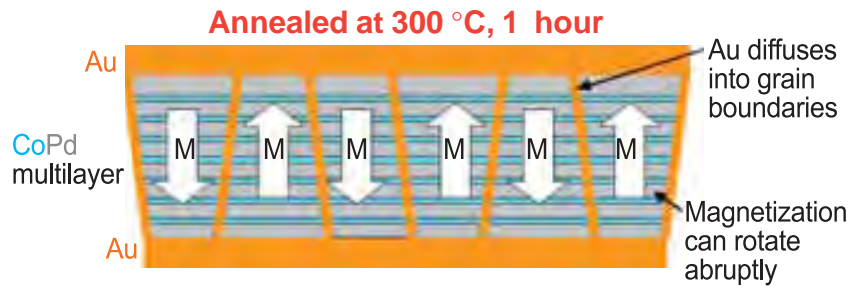

Figure 2: Grains isolated magnetically by Au diffusing into grain boundaries.

In recent work, we have found a novel method for magnetically decoupling grains in CoPd media. CoPd multilayers are one of the leading candidates for the next generation of magnetic media. We have found that if we deposit an Au film on top of the CoPd and anneal the sample in air, two unexpected phenomena occur as a result of rapid diffusion of atoms along grain boundaries. One is that Co atoms diffuse to the surface, react with oxygen, and remain at the surface. The other is that $\mathrm{Au}$ atoms diffuse into the grain boundaries replacing Co. The net effect is that the grain boundaries are demagnetized, and each grain can magnetically switch independently of its neighbors. This method provides the best magnetic isolation of grains yet found.

This work has been reported at several conferences and industrial workshops.

\section{Contributors and Collaborators}

E.B. Svedberg, D. Weller (Seagate); R.D. McMichael, T.P. Moffat, J. Mallett, A.J. Shapiro, J.E. Bonevich (Metallurgy Division, NIST); D.P. Pappas

(Electromagnetics Division, NIST); M.D. Stiles

(Physics Lab, NIST) 


\section{Advanced Manufacturing Processes}

The competitiveness of U.S. manufacturers depends substantially on their ability to create new product concepts and to quickly translate such concepts into manufactured products that meet their customers' increasing expectations of performance, cost, and reliability. This is equally true for well-established "commodity" industries, such as automotive, aerospace, and electronics; for materials suppliers of aluminum, steel, and polymers; and for rapidly growing industries based on nanotechnology and biotechnology. In support of these industries, MSEL is developing robust measurement methods, standards, software, and process and materials data needed for design, monitoring, and control of new and existing materials and their manufacturing processes. The Advanced Manufacturing Processes Program focuses on the following high-impact areas:

- Development of combinatorial and high-throughput methods for developing and characterizing materials ranging from thin films and nanocomposites to micro and macroscale materials structures;

- Automotive industry-targeted R\&D for improved measurement methods for sheet metal forming of lightweight metals and for the development of hydrogen storage materials needed for hydrogen-powered vehicles;

- Development of innovative, physics-based process modeling tools for simulating phase transformations and deformation during manufacturing and creation of the databases that support such simulations;

- National traceable standards having a major impact on trade, such as hardness standards for metals and MALDI process standards for polymers; and

- Development of innovative microfluidic testbeds for process design and characterization of polymer formulations.

Our research is conducted in close collaboration with industrial partners, including industrial consortia, and with national standards organizations. These collaborations not only ensure the relevance of our research, but also promote rapid transfer and utilization of our research by our partners. Three projects from the Advanced Manufacturing Methods Program are highlighted below.

\section{NIST Combinatorial Methods Center (NCMC)}

The NCMC develops innovative combinatorial and high-throughput (C\&HT) measurement techniques and experimental strategies for accelerating the discovery and optimization of complex materials and products, such as polymer coatings and films, structural plastics, fuels, personal care goods, and adhesives. These C\&HT array and gradient methods enable the rapid acquisition and analysis of physical and chemical data from materials libraries, thereby accelerating materials discovery, manufacturing design, and knowledge generation. In 2005, the NCMC Consortium consisted of 19 institutions from industry, government laboratories, and academic groups, which represents a broad cross-section of the chemical and materials research sectors. A growing component of the NIST NCMC program is focused on accelerating the development and understanding of emerging technologies, including nanostructured materials, organic electronics, and biomaterials, and, in particular, on the nanometrology needed for C\&HT-based research for these technologies.

\section{Forming of Lightweight Materials}

Automotive manufacturing is a materials intensive industry that involves approximately $10 \%$ of the U.S. workforce. In spite of the use of the most advanced, cost-effective technologies, this globally competitive industry has major productivity issues related to materials measurements, materials modeling, and process design. Chief among these is the difficulty of designing stamping dies for sheet metal forming. An ATP-sponsored workshop ("The Road Ahead," June 20-22, 2000) identified problems in the production of working die sets as the main obstacle to reducing the time between accepting a new design and actual production of parts. This is also the largest single cost (besides labor) in car production. Existing finite element models of deformation and the materials measurements and data on which they are based are inadequate to the task of evaluating a die set design: they do not accurately predict the multi-axial hardening, springback, and friction of sheet metal during metal forming processes and, therefore, the stamping dies designed using finite element analyses must be modified through physical prototyping to produce the desired shapes, particularly for high-strength steels and aluminum alloys. To realize the weight savings and increased fuel economy enabled by high-strength steel and aluminum alloys, a whole new level of formability measurement methods, models, and data is needed for accurate die design, backed by a better understanding of the physics behind metal deformation. The MSEL Metallurgy Division is working with the U.S. automakers and their suppliers to fill these needs. A key component of our program is the unique multi-axial deformation measurement facility with which local strains in deformed metal sheet can be measured in situ. This facility has enabled NIST to take a key role in developing new methods for 
assessing springback, residual stresses, friction between the sheet metal and die during forming, and surface roughening, and in providing benchmark data for international round-robin experiments for finite element code. New techniques for detecting local deformation events at surfaces are providing insights into the physics of deformation and are leading to physics-based constitutive equations.

\section{Hardness Standardization: Rockwell, Vickers, and Knoop}

Hardness is the primary test measurement used to determine and specify the mechanical properties of metal products and, as such, determines compliance with customer specifications in the national and international marketplace. The MSEL Metallurgy Division is engaged in developing and maintaining national traceability for hardness measurements and in assisting U.S. industry in making measurements compatible with other countries around the world, enabled through our chairing the ASTM International Committee on Indentation Hardness Testing and heading the U.S. delegation to the ISO Committee on Hardness Testing of Metals, which oversees the development of the organizations' respective hardness programs. Our specific R\&D responsibilities include the standardization of the national hardness scales, development of primary reference transfer standards, leadership in national and international standards writing organizations, and interactions and comparisons with U.S. laboratories and the National Metrology Institutes of other countries.

Contact: Frank W. Gayle (Metallurgy Division) 


\section{Mechanisms for Delivery of Thermodynamic and Kinetic Data}

Many commercial processes are controlled by the thermodynamic and diffusion properties of the material. The ready availability of reliable data is a key component to successful design of new materials and manufacturing processes. Thermodynamic and diffusion mobility databases provide an efficient method of storing the wealth of these data, and software tools allow the user to efficiently retrieve the needed information.

\section{Ursula R. Kattner and Carelyn E. Campbell}

$\mathrm{T}$ he complexity of thermodynamics and diffusion kinetics prevented their direct application in the design of complex materials and processes in the past. Graphical representation of multicomponent systems is too complex, and, because of the enormous number, storage of every single datum is impractical. However, mathematical functions representing thermodynamic and diffusion properties of the phases permit efficient storage. Since these functions are based on physical models, they further provide the power to extrapolate binary and ternary systems to higher order systems. Software then allows calculation of the desired quantities. This approach is called the CALPHAD methodology. Unfortunately, previous software was tailored for expert users and was difficult for the occasional, less experienced user.

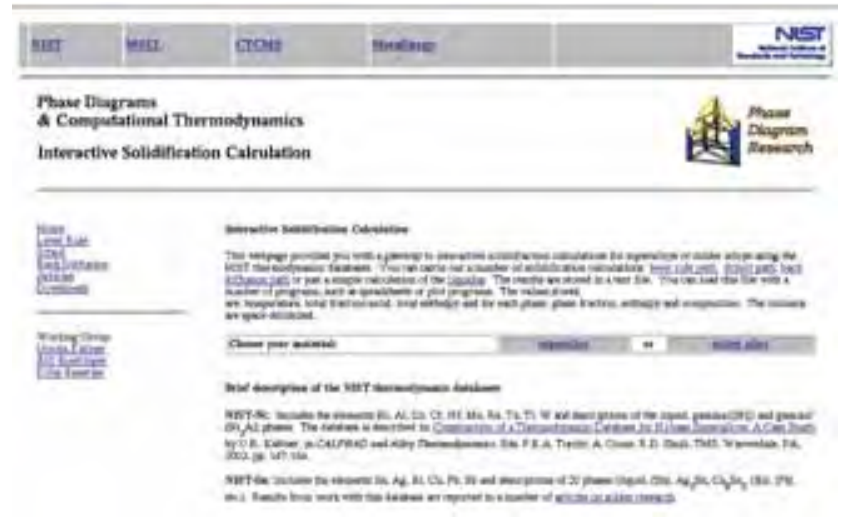

Figure 1: Web site for interactive solidification calculations.

The databases and software developed in this project are designed to provide users with simple tools to retrieve and disseminate information needed for efficient materials and process design. A web site is being developed for interactive solidification calculations using the NIST superalloy and solder thermodynamic databases (Figure 1). Results are provided in tabular form and can be used with other software packages, such as Mathematica or spreadsheets, for further manipulation. Links to a downloadable Mathematica script for DTA analysis simulation using these output tables as well as tutorial pages on the interpretation of phase equilibria information are also found on the Metallurgy Division Phase Diagram web site (www.metallurgy.nist.gov/phase/).

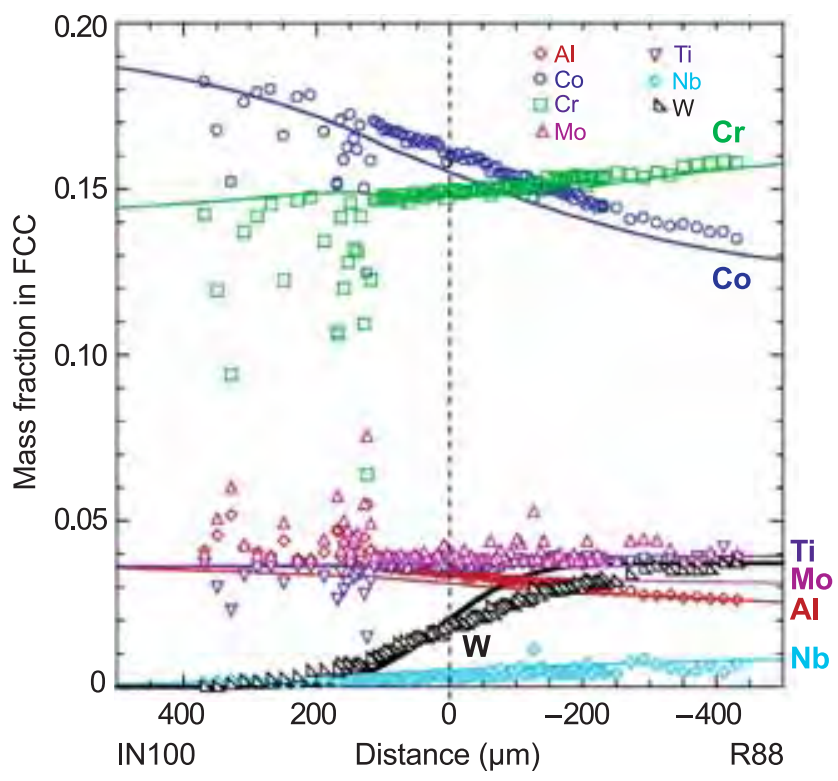

Figure 2: Experimental and calculated composition profiles for IN100/René-88 diffusion couple $\left(1150^{\circ} \mathrm{C}, 1000 \mathrm{~h}\right)$.

Multicomponent multiphase diffusion couples can be simulated using NIST thermodynamic and diffusion mobility databases, and experimental and calculated composition profiles can be compared. Figure 2 shows the comparison of calculated and experimental composition profiles for a $\gamma+\gamma^{\prime} / \gamma$ diffusion couple (IN100/René-88). The high fraction of $\gamma^{\prime}$ in the IN100 alloy leads to the observed experimental scatter on the left-hand side. Efforts to incorporate the diffusion in ordered phases such as $\mathrm{B} 2$ (NiAl) and $\gamma^{\prime}\left(\mathrm{Ni}_{3} \mathrm{Al}\right)$ are being pursued. Future work will assess the diffusion mobilities in the Ni-Al-Cr system for the B2 and $\gamma^{\prime}$ phases.

Interactions developed through our "High Throughput Analysis of Multicomponent Multiphase Diffusion” workshop series are posted at (www.ctcms.nist.gov). The NIST Diffusion Data Center is being converted into an electronic searchable form, and a preliminary version is now available at < winweb.nist.gov/diffusion>.

\section{Contributors and Collaborators}

W.J. Boettinger (Metallurgy Division, NIST); A.R. Roosen (CTCMS, NIST); J.C. Zhao (General Electric) 


\section{FiPy: An Adaptable Framework for Phase Field and Level Set Modeling}

Phase field and level set methods have enjoyed considerable success for phase transformation modeling. However, any but the simplest implementations of these methods are beyond the capabilities of otherwise interested researchers. An adaptable software tool is being developed to support these scientists.

\section{Jonathan E. Guyer and Daniel Wheeler}

$\mathrm{T}$ The equations that model the evolution of many physical, chemical, and biological systems have a remarkably similar form. Despite the common mathematical structure of these models and the common approaches to solving them, most research codes are developed by individuals for one particular problem and then abandoned. They tend not to be documented well enough nor designed well enough to be adapted by other scientists. In fact, many scientists have difficulty adapting their own previous codes and opt, instead, to start from scratch for each new problem.

Another problem with research codes is that they are often implemented with the solution schemes that result in a functioning code in the shortest amount of time. As a result, little advantage is taken of sophisticated numerical techniques that could allow dramatically faster solutions, over larger physical domains, for longer elapsed simulation times.

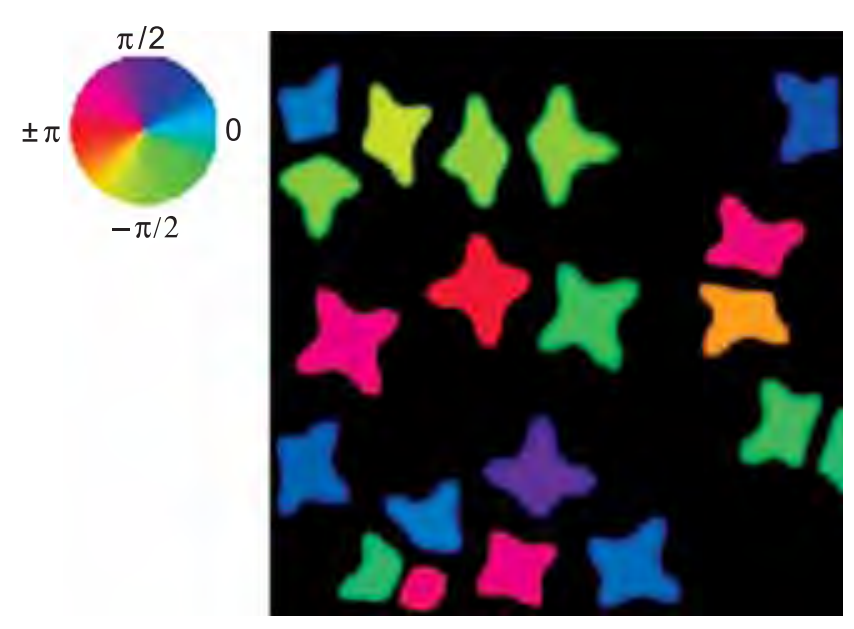

Figure 1: Phase field calculations of anisotropic grain growth.

A software framework called FiPy has been jointly developed in the Metallurgy Division and the Center for Theoretical and Computational Materials Science (CTCMS) to address this constant reinvention of phase transformation codes. FiPy is based on the finite volume method of solving PDEs, which is widely employed in the demanding field of computational fluid dynamics. The goal of the project has been to encapsulate existing libraries and tools for tasks such as sparse matrix solutions and data visualization into a framework that is approachable by materials scientists. At the same time, every aspect of the tool is open, allowing any user to adapt, extend, or improve as they desire.

First released in November 2004, FiPy is already being used for phase field work by researchers at NIST and elsewhere. Existing codes have been re-implemented in FiPy, making them easier to understand and opening them to a wider battery of solution methods. FiPy has also enabled prototyping new problems in a matter of hours, rather than the days or weeks that were previously required. In addition, FiPy is being applied to new models of both cell chemotaxis and drug delivery.

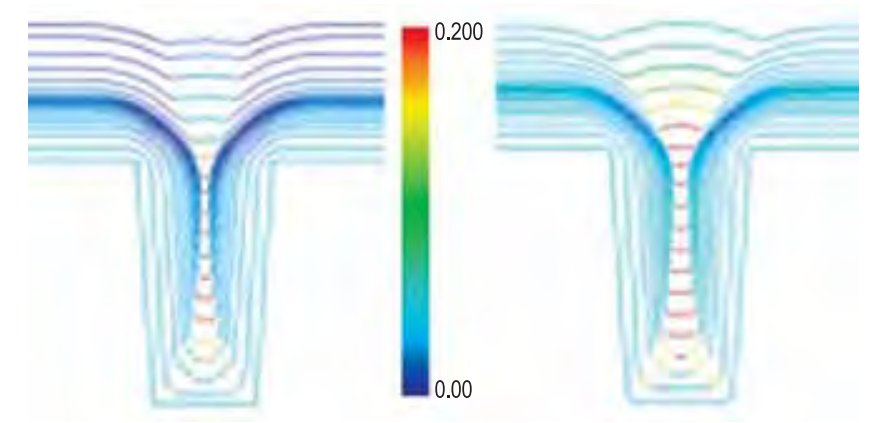

Figure 2: Level set calculations of "superfill."

Perhaps the greatest success of the tool has been to allow dissemination of NIST's Curvature Enhanced Accelerator Coverage (CEAC) model to researchers outside of NIST. This model of electrochemical "superfill” has successfully explained many aspects of the metallization of microchips, however the FORTRAN-based level set code to implement the model was impractical to use by anyone other than its author. Since being re-implemented in FiPy, the code has already been put into use by both industrial and academic researchers. The FiPy implementation of CEAC was highlighted by John Dukovic of Applied Materials in his Electrochemical Research Award Address at the 2004 Electrochemical Society meeting. FiPy can be obtained via the web at http://www.ctcms.nist.gov/fipy.

\section{Contributors and Collaborators}

J. Warren (Metallurgy Division and CTCMS, NIST); D. Saylor (FDA); W. Losert (University of Maryland); J. Dukovik (Applied Materials); Y. Shacham-Diamand (Tel-Aviv University and Waseda University); D. Lewis (General Electric) 


\section{Metrology Tools to Accelerate Industrial Development of Solid State Hydrogen Storage Materials}

\begin{abstract}
Realization of the "Hydrogen Economy" will require technological advances in hydrogen production, storage, and fuel cell implementation. Hydrogen storage poses the greatest technical challenge, and solid-state storage of hydrogen in metal hydrides will eventually be required to alleviate the safety issues associated with on-board vehicular storage of high pressure or liquefied hydrogen. The current state of metal hydride storage technology falls far short of targets for vehicular applications. A suite of versatile measurement tools is needed to accelerate the development of hydrogen storage materials.
\end{abstract}

\section{Leonid A. Bendersky, Ursula R. Kattner, and Martin L. Green}

$\mathrm{T}_{\mathrm{n}}^{\mathrm{h}}$ he hydrogen economy has enormous potential to make the United States less dependent on foreign sources of energy, as well as create a healthier global environment. In 2003, the hydrogen-powered "Freedom Car" was put forth as a grand challenge to the American people, and funded at the level of \$1.2B. Of the three enabling technologies necessary for the realization of this vehicle, i.e., hydrogen production, storage, and fuel cell implementation, hydrogen storage is viewed as the biggest obstacle.

On-board storage of high-pressure or liquefied hydrogen, the most direct approach, has insurmountable safety issues. In addition, neither liquid hydrogen (8.4 MJ/L) nor high-pressure (133 MPa) hydrogen $(4.4 \mathrm{MJ} / \mathrm{L})$ meet the fuel energy density specification of $10 \mathrm{MJ} / \mathrm{L}$ established for the Freedom Car. Solid-state storage of hydrogen in metal hydrides is a safer alternative for automobiles, as well as for ships, aircraft and spacecraft. However, the development of solid-state hydrogen storage materials poses many challenges, and current materials fall far short of the Freedom Car goals.

Major limitations of metal hydride storage are as follows: energy density is presently limited to about $4 \mathrm{MJ} / \mathrm{L}$; desorption behavior (delivery) is not sufficiently rapid; excessive heat is evolved as a result of hydrogen adsorption ("filling up"); the fundamental thermochemistry of hydrides and hydrogen adsorption and desorption kinetics are a poorly understood function of composition and microstructure; finally, reliability of the hydrides, i.e., fatigue and degradation during reversible hydrogen cycling, is not known.

The goal of this project is the development of new high-throughput measurement methods to study this extremely important phase transformation in a wide variety of metal and intermetallic-based hydrides, thereby providing tools for industry to develop these materials at a greatly accelerated pace.

A multidisciplinary approach is used to address some of the major problems associated with solid-state hydrogen storage: (i) development of IR emission spectroscopy and microcalorimetry-based high-throughput metrologies for measuring hydrogen adsorption and desorption kinetics, as well as thermochemical parameters of metal hydrides; and (ii) development of thermodynamic and kinetic data underlying this behavior, to guide future development.

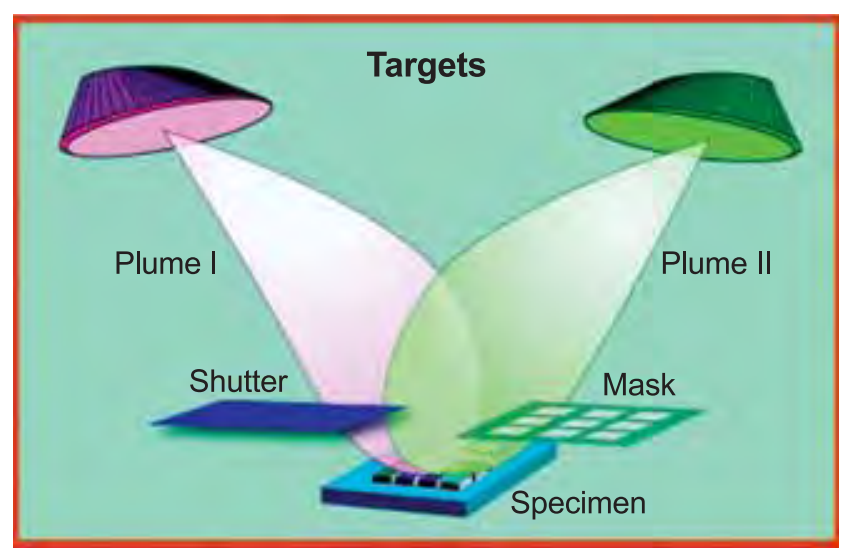

Figure 1: Synthesis of combinatorial continuous compositional spreads.

During the initial phase of this project, combinatorial film libraries of a metal alloy system have been fabricated in a form of thin film continuous compositional spreads. Initial systems include: $\mathrm{Fe}_{2} \mathrm{Ti}_{-}-\mathrm{FeTi}_{2}$, Ni-Mg, and $\mathrm{Zr}_{35} \mathrm{Ni}_{65-\mathrm{x}} \mathrm{V}_{\mathrm{x}}$. The synthesis of the compositional spread is verified by EDS, $x$-ray diffraction, and cross-sectional TEM. The construction of the reaction chamber for the hydrogenation and IR measurement experiments has been initiated. Special precautions were made in the design to address safety issues arising from working with hydrogen gas at elevated temperatures and pressures.

For the construction of the thermodynamic database, the available Gibbs energy functions of the alloy systems and the metal hydride are also being evaluated.

\section{Contributors and Collaborators}

D. Josell (Metallurgy Division, NIST); L.P. Cook (Ceramics Division, NIST); E.J. Heilweil (Physics Lab, NIST); S. Semancik (CSTL, NIST) 


\section{Fundamental Nature of Crack Tips in Glass}

Lifetimes of glasses and structural ceramics are determined by the size and severity of mechanical flaws and defects found in their surfaces. Understanding the nature of these flaws and how they respond to the surrounding chemical and physical environment is of particular importance for the prediction of component lifetime. This project has as its objectives the quantification of crack growth parameters in glasses and ceramics in order to provide design information to colleagues (NASA, SSG Inc.) wanting to improve the reliability of real components. Thus the project provides crack growth parameters for glasses and ceramics, characterizes cracks and crack tips in these materials using advanced detection techniques, and develops methods of assuring structural reliability.

\section{Sheldon M. Wiederhorn}

$\mathrm{T}_{\mathrm{f}}^{\mathrm{h}}$ he static fatigue limit in glasses is important for predicting the long-term reliability of glass. The static fatigue limit is the applied stress intensity factor (load times the square root of crack length) below which cracks will no longer grow. Some glasses (soda-lime-silica glass) have a static fatigue limit; others (silica) do not. The methodology for assuring structural reliability can depend on which kind of glass is being considered for an application. Hence, it is important to characterize the factors that determine the effectiveness of this limit in controlling fracture.

Using atomic force microscopy, we characterized crack shape and other features that develop at crack tips in glass near the static fatigue limit. Both of these characterizations require measurements at the nanometer scale. The techniques developed on this project are proving very promising to explore the high-stress region at crack tips.

During the past year, we concluded that the fatigue limit is a consequence of the exchange of alkali ions in the glass for hydroxyl ions in the external environment. The ion exchange results in a compressive stress around the crack tip that retards crack motion when the load is increased above the fatigue limit. Although the flanks of the crack are corroded, the corrosion does not reach the crack tip, which is held closed by the compressive stresses arising from the ion exchange process.

Finally, fracture areas taken from opposite sides of the fracture surface have been shown to match to an accuracy of $0.3 \mathrm{~nm}$ normal to the surface and $5 \mathrm{~nm}$ parallel to the surface. No evidence of cavitation is observed, which means that the fracture of glass occurs by a completely brittle process. This observation is contrary to mechanisms reported for very brittle bulk metallic glasses, for which cavity formation precedes the fracture process in the glass.
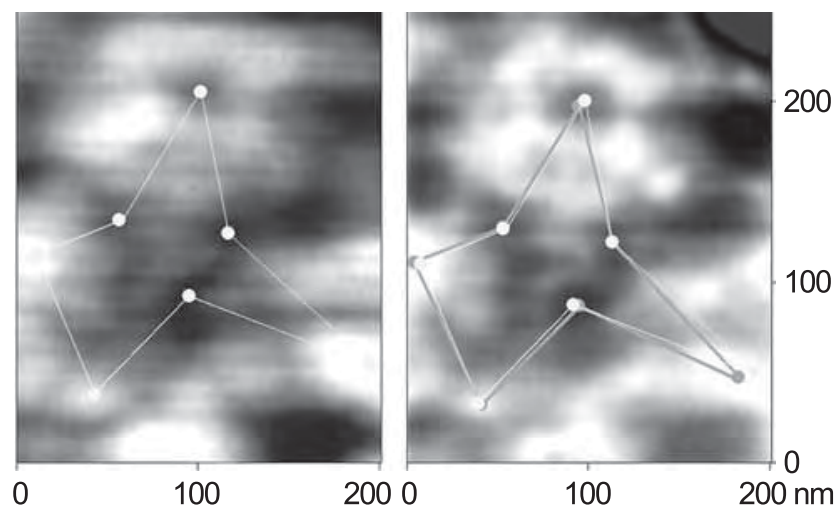

Figure 1: A comparison of opposing fracture surfaces of soda-lime-silicate glass, AFM scan. The two surfaces are scanned separately. The sections of the surface are placed in the figure in such a way that the highlights in the two figures match. Note the overlap of the polygons formed by connecting the same highlight features in each figure. Within experimental scatter, the two images are identical.

The difference in behavior between silicate glasses and bulk metallic glasses is probably related to the type of bond holding the material together. Bonds in silicate glasses are covalent in nature. These are directional and resistant to shear. By contrast, bonding in metallic materials is non directional, which makes them susceptible to displacements by shear stresses, hence the ease of cavity formation at the tips of cracks in bulk metallic glasses.

\section{Publications}

Jean-Pierre Guin and Sheldon M. Wiederhorn, "Fracture of Silicate Glasses: Ductile or Brittle?" Phys. Rev. Let. 92 [21] 215502 (2004).

Jean-Pierre Guin, Sheldon M. Wiederhorn, and Theo Fett, "Crack-Tip Structure in Soda-Lime-Silicate Glass: Experimental Observations,” J. Am. Ceram. Soc. 88 [3] 652-659 (2005).

\section{Contributors and Collaborators}

Prof. Tanguy Rouxel (University of Rennes, France); Prof. Theo Fett (Forschungszentrum, Karlsruhe, Germany) 


\section{Hardness Standardization: Rockwell, Vickers, Knoop}

Hardness is the primary test measurement used to determine and specify the mechanical properties of metal products. The Metallurgy Division is engaged in all levels of standards activities to assist U.S. industry in making hardness measurements compatible with other countries around the world. These activities include the standardization of the national hardness scales, development of primary reference transfer standards, leadership in national and international standards writing organizations, and interactions and comparisons with U.S. laboratories and the National Metrology Institutes of other countries.

\section{Carlos Beauchamp and Sam Low}

Al the international level, the Metallurgy Division is leading the Working Group on Hardness (WGH) under the International Committee for Weights and Measures (CIPM), with a goal to standardize hardness measurements worldwide. As Secretary of the WGH, we have led an effort this year to better define the Rockwell hardness test procedure used by National Metrology Institutes (NMIs). The new definition is awaiting approval by the CIPM. Other activities include chairing the ASTM-International Committee on Indentation Hardness Testing and heading the U.S. delegation to the ISO Committee on Hardness Testing of Metals, which oversees the development of the organizations' respective hardness programs.

In conjunction with ASTM International and IMEKO, the Metallurgy Division organized the HARDMEKO 2004 Conference, an international conference for scientists and specialists involved in the measurement of hardness (see Figure 1). The conference was very successful, bringing together an international audience to hear over 25 technical presentations by experts from 11 countries.

Our primary task at the national level is to standardize the U.S. national hardness scales and to provide a means of transferring these scale values to industry. Currently, we are producing test block Standard Reference Materials ${ }^{\circledR}$ (SRMs) for the Rockwell, Vickers, and Knoop hardness scales, as well as developing new reference standards. A new microindentation standardizing machine has been acquired and is currently undergoing testing and uncertainty analysis. Vickers and Knoop microindentation SRMs are available at hardness levels of 125,600 , and $760 \mathrm{kgf} / \mathrm{mm}^{2}$. Additional hardness levels are being investigated ranging from 900 to $1,400 \mathrm{kgf} / \mathrm{mm}^{2}$. These blocks will significantly extend the range of microindentation SRMs offered by NIST.

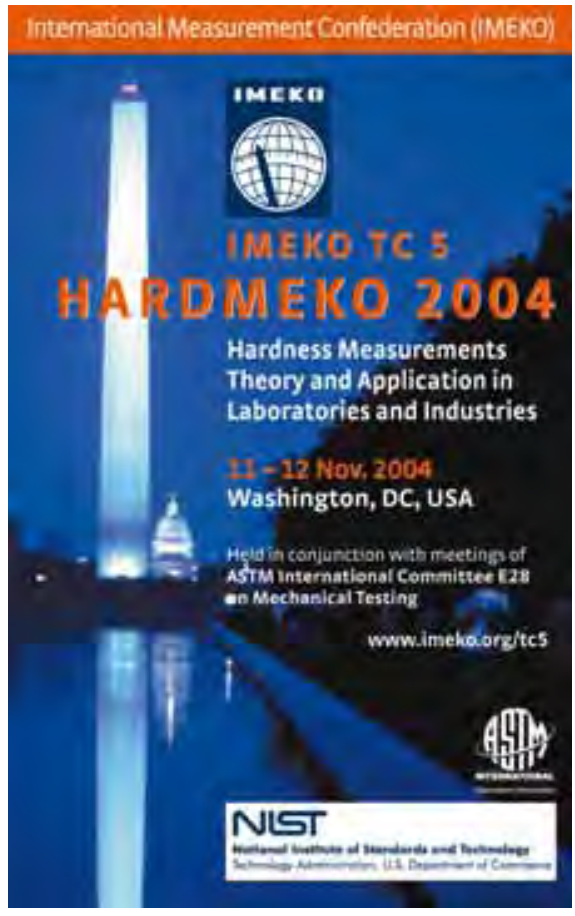

Figure 1: HARDMEKO 2004 Conference organized by NIST with ASTM and IMEKO.
In addition to newly introduced Standard Reference Materials ${ }^{\circledR}$ (SRMs) for the Rockwell B scale and three SRM Rockwell C scale blocks currently available, we are in the process of producing new SRMs for the HR15 N and HR30 N Rockwell scales. The N scales are used primarily for measuring thinner gage material or case depth. Work is also continuing to produce a new Rockwell hardness diamond indenter SRM. A joint project with Gilmore Diamond Tools has recently demonstrated that diamond indenters can be repeatedly produced with the correct shape and adhere to the very tight calibration grade tolerances.

Other activities at the national level occurring this year included the assessment of commercial secondary hardness calibration laboratories for the NIST National Voluntary Laboratory Accreditation Program (NVLAP) and the development of indentation Finite Element Analysis models used to analyze effects of different indenter materials for all Rockwell hardness scales in support of proposed revisions to international test standards.

\section{Contributors and Collaborators}

J. Fink, D. Kelley, L. Ma, H. Gates (Metallurgy Division, NIST); J. Song (MEL, NIST); W. Liggett, Jr., N. Zhang (ITL, NIST); S. Doty, B. Belzer, D. Faison (NVLAP, NIST); W. Stiefel (TS, NIST); M. Mihalec (Gilmore Diamond Tools, Providence, RI) 


\section{NIST Combinatorial Methods Center Pioneer and Partner in Accelerated Materials Research}

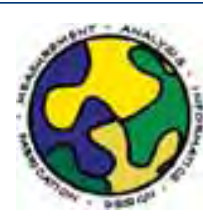

\begin{abstract}
Combinatorial and high-throughput (C\&HT) methods hold great potential for making materials research more productive, more thorough, and less wasteful. However, significant barriers prevent the widespread adoption of these revolutionary techniques. Through creative, cost-effective measurement solutions, and with an eye towards fruitful collaboration, the NIST Combinatorial Methods Center (NCMC) strives to ease the acquisition of C\&HT techniques by the materials research community.
\end{abstract}

\section{Michael J. Fasolka}

$\mathrm{T}$ he NIST Combinatorial Methods Center is now in its fourth year of service to industry, government laboratories, and academic groups interested in acquiring C\&HT research capabilities for materials research. In 2005, the NCMC consortium included 19 member institutions (see table), which represent a broad crosssection of the chemical and materials research sectors.

The NCMC fosters wide-spread adaptation of C\&HT technologies through two complementary efforts. The first is an extensive research program, centered in the Multivariant Measurement Methods Group of the NIST Polymers Division. Our research provides innovative measurement solutions that serve to accelerate the discovery and optimization of complex products such as polymer coatings and films, structural plastics, fuels, personal care goods and adhesives. Moreover, a growing component of our program aims to speed the development and understanding of emerging technologies including nanostructured materials, nanometrology, organic electronics, and biomaterials. Several of these research directions are highlighted elsewhere in this report, as identified by the NCMC symbol (see top right).

\begin{abstract}
"The coatings industry has been traditionally perceived to react slowly to implementing newer and quantifiable measurement techniques for characterizing structure-property relationships in paints and films. The [NCMC] has pioneered elegant approaches that can significantly reduce experimental time [for] testing coating formulation performance."
\end{abstract}

- D. Bhattacharya (Eastman Chemical)

In conjunction with its research program, the NCMC conducts an outreach effort to disseminate NIST-developed C\&HT methods, assess industry measurement needs, and form a community to advance the field. A key component of NCMC outreach is our series of member workshops. On November 8-9 2004, we hosted our 6th workshop, NCMC-6: Advanced Materials Forum. The goal of NCMC-6 was to gauge emerging industrial needs for C\&HT measurements of materials systems such as biomaterials and organic electronics. Accordingly, NCMC-6 included plenary symposia outlining engineering issues in these advanced systems, sessions illustrating NIST capabilities in these areas, and a panel discussion aimed at determining new measurements that should be pursued.

In addition, on May 2-3 2005, we hosted NCMC-7: Adhesion and Mechanical Properties II. Central to this event was a symposium presenting new NCMC methods for the development and optimization of adhesives. Highlights included a new gradient peel-test for the HT assessment of backed adhesives (e.g., tapes), and approaches for the rapid screening of epoxy formulations. A variation of our buckling technique to measure modulus, useful for evaluating soft systems such as polymer gels, was also described.

\section{"As a member of NCMC, I believe that Procter \& Gamble has access to a high-performance work group with expertise in high throughput and combinatorial techniques. The conferences have been particularly valuable for networking with NIST scientists as well as other industrial members of NCMC." — M. McDonald (Procter and Gamble)}

Moreover, this year the NCMC continued community forming activities by organizing several high-profile sessions dedicated to C\&HT research at national conferences, including meetings of the American Physical Society, the American Chemical Society, the Materials Research Society, and the Adhesion Society.

"The combinatorial methods program at NIST makes the NCMC critical to any company's development of high-throughput workflows. The import of this effort to industry is clearly indicated by your center's number of industrial members." — J. Dias (ExxonMobil)

For more information on the NIST Combinatorial Methods Center, please visit http://www.nist.gov/combi.

NCMC Members (*New in FY2005):

Air Force Research Lab
Air Products \& Chemicals
Arkema Inc.
BASF
Bayer Polymers
BP
Dow Chemical Company
Eastman Chemical
ExxonMobil Research
Honeywell International

\section{Contributors and Collaborators}

C.M. Stafford, P.M. McGuiggan, K.L. Beers, A. Karim, E.J. Amis (Polymers Division, NIST) 


\section{Polymer Formulations: Materials Processing and Characterization on a Chip}

We develop high-throughput methods to advance polymer formulations science through the fabrication of microscale instrumentation for measuring physical properties of complex mixtures. Adaptation of microfluidic technology to polymer fluid processing and measurements provides an inexpensive, versatile alternative to the existing paradigm of combinatorial methods. We have built a platform of polymer formulations-related functions based on modified microfluidic device fabrication methods established in our facilities.

\section{Kathryn L. Beers}

$\mathrm{M}$ icrofluidic device fabrication methods previously developed in the Polymers Divison enable combinatorial fabrication and characterization of polymer libraries. Recent accomplishments include the integration of multiple functions on a chip for the formulation, mixing, processing, and characterization of polymer particles for evaluation of dental composite materials and the fabrication of gradient polymer brush surfaces for measuring the behavior of stimuli-responsive surfaces. (b)

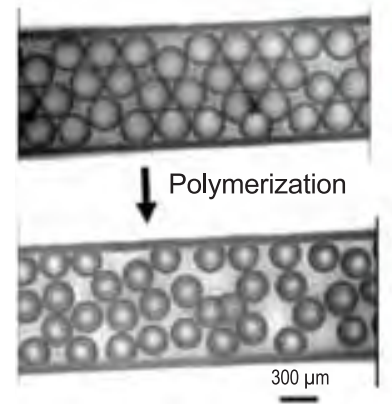

(a)

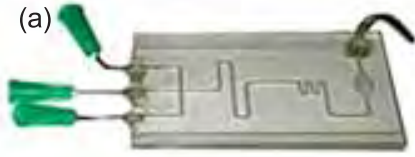

(c)

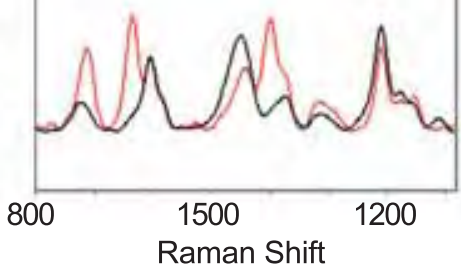

Figure 1: (a) A thiolene microfluidic device used to create, mix, polymerize and characterize monomer droplets.

(b) Optical images of monomer droplets and polymer particles.

(c) Raman spectra of monomer (red) and polymer (black).

Building on our ability to form organic-phase droplets in thiolene-based microfluidic devices (Figure 1a), we can establish libraries of droplets with systematic composition variations. The droplets are subject to various processes such as mixing and photopolymerization on the chip. Raman spectroscopy on the chip (Figure 1c) and optical imaging (Figure 1b) are used to measure and correlate properties such as monomer composition and conversion to polymer with shrinkage. The first publication on this work (Langmuir 21, 3629, 2005) was recently profiled in the Research Highlights of Lab on a Chip.

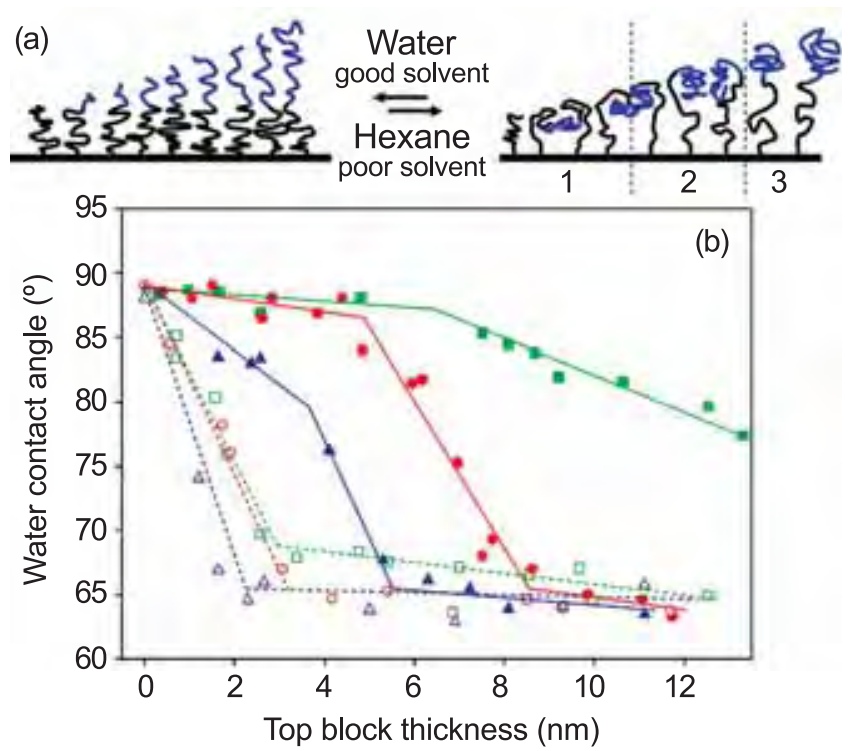

Figure 2: (a) Schematic of amphiphilic block copolymer brush gradients representing the proposed conformation shift in response to good and poor solvents for the top block layer. (b) Water contact angle measurements as a function of top block thickness on three gradients of top block thickness on uniform bottom blocks of three different lengths (blue - $4 \mathrm{~nm}$, red - $10 \mathrm{~nm}$, green - $14 \mathrm{~nm}$ ) in good (open) and poor (filled) solvents for the top block.

Microchannel confined surface initiated polymerization was used to prepare surfaces with gradients of molecular weight and block and statistical copolymer composition. The block copolymer surfaces were studied for their ability to reorganize at the air/solution interface depending on the nature of the polymer and solvent (Figure 2a). The ability of the surface layer to rearrange was shown to depend on the thickness of both the top and bottom block layers (Figure 2b).

The capabilities for controlled radical polymerization on a chip (CRP Chip) were also extended this year to include block copolymer synthesis and higher-order control of solution compositions. A three-input device was developed, enabling stoichiometric variations in reactions and faster measurement of kinetic behavior (Macromol. Rapid Commun. 26, 1037, 2005).

\section{Contributors and Collaborators}

Z.T. Cygan, C. Xu, S. Barnes, T. Wu, A.J. Bur, J.T. Cabral, S.D. Hudson, A.I. Norman, J. Pathak, W. Zhang, M.J. Fasolka, E.J. Amis (Polymers Division, NIST) 


\section{Quantitative Polymer Mass Spectrometry}

\author{
Matrix-assisted laser desorption ionization \\ time-of-flight mass spectrometry (MALDI-TOF-MS) \\ is being developed as a method for absolute \\ molecular mass distribution measurement of \\ synthetic polymers. This means determining a \\ comprehensive uncertainty budget for a complex \\ measurement technique that must include both \\ Type A ("random") and Type B ("systematic") \\ uncertainties.
}

\section{William E. Wallace}

Tn mass spectrometry, methods exist to calibrate 1 the mass axis with high precision and accuracy. In contrast, the ion-intensity axis is extremely difficult to calibrate. This leads to large uncertainties in quantifying the content of mixtures. This is even true when the mixture is composed solely of different mass oligomers of the same chemical species as in the case of polymer polydispersity. The aim of this project is to calibrate the ion intensity axis. This task has been divided into three parts: sample preparation/ion production, instrument optimization/ion separation, and data analysis/peak integration. Each part is necessary but on its own is not sufficient to guarantee quantitation.

We study the MALDI ion-creation process phenomenologically using combinatorial libraries. The ratio of analyte to matrix is varied along a linear path laid down by nebulizing a continuously varying mixture of two solutions, one analyte + matrix + salt and the other matrix + salt. In our case, the analyte is a mixture of two polymers having different end groups and closely matched molecular mass distributions. The figure on this page shows such a library where the blue arrows indicate a linearly changing analyte: matrix ratio.

To this, we add stochastic-gradient numerical optimization to adjust the instrument parameters at each composition to give a mass spectrum that best matches the known polymerA:polymerB ratio in the analyte. Instrument parameters optimized include laser energy, ion extraction voltage, ion lens voltage, extraction delay time, and detector voltage. Stochastic methods must be used because the data has some measure of Type A random uncertainty (i.e., "noise") to it; therefore, exact values of the function to be optimized are not available.

Finally, to this we add our MassSpectator software which ensures unbiased, logically-consistent integration of the peaks in the (noisy) mass spectrum. A software script has been written around MassSpectator that automatically identifies oligomeric series in the mass spectrum and calculates the total amount and the molecular mass moments for each series identified.

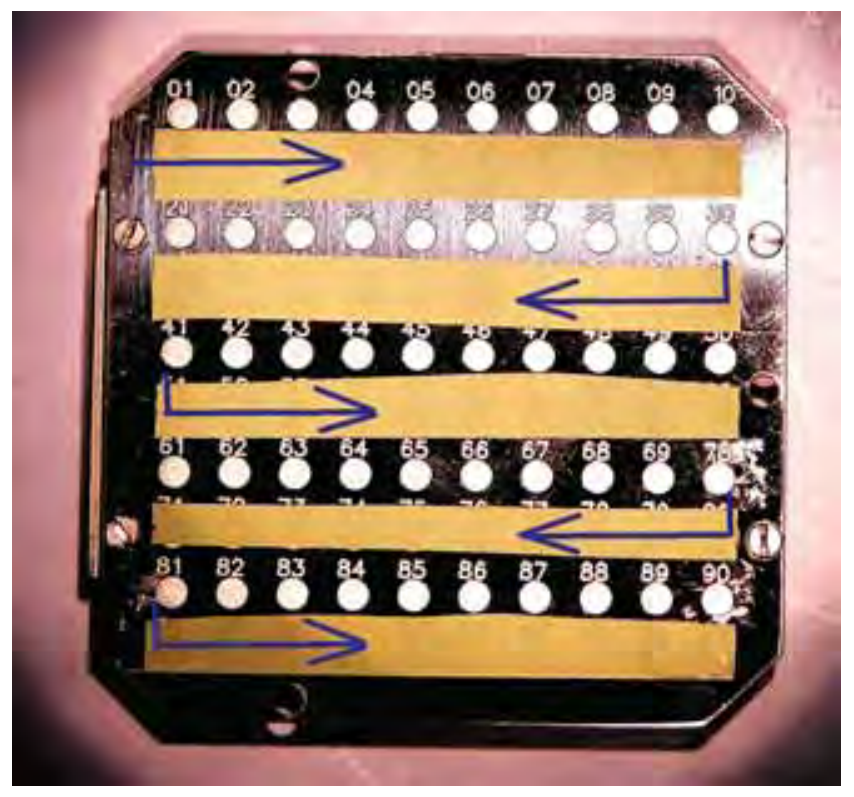

An early embodiment of our approach can be found in ASTM Standard Test Method D7134, the first MALDI-TOF-MS method endorsed by ASTM. Working through the Versailles Project on Advanced Materials and Standards (VAMAS), and in close cooperation with our industry and national metrology institute (NMI) colleagues from around the world, an interlaboratory comparison was initiated to understand the nexus of critical measurement factors when performing quantitative polymer mass spectrometry. From the knowledge gained by the interlaboratory comparison, D7134 was written with particular attention paid toward controlling the critical factors.

The MALDI project maintains a vigorous, worldwide outreach program including an online polymer MALDI recipes catalog, annual polymer MS workshops, and the availability of our MassSpectator software on the web. For more information on any of these topics please visit our web page at: www.nist.gov/maldi.

\section{Contributors and Collaborators}

W.R. Blair, K.M. Flynn, C.M. Guttman (Polymers Division, NIST); A.J. Kearsley (Mathematical \& Computational Sciences Division, NIST) 


\section{Standard Tests and Data for Sheet Metal Formability}

In order to meet goals for fuel efficiency, the U.S. automotive industry is moving to lighter, high-strength materials for auto bodies. NIST has surveyed the industry and found that accurate material properties and methods for die designers to incorporate them into finite element models of sheet-metal forming dies, is a critical need for the U.S. auto industry. This project seeks to develop new standard tests and metrology to accurately determine sheet metal mechanical response under forming conditions.

\section{Timothy J. Foecke and Mark ladicola}

uccessful introduction of new high-performance $\mathcal{S}$ alloys into automotive production requires comprehensive data on alloy behavior under realistic forming conditions. Existing tests are inadequate. This project is developing two sheet metal formability tests, along with associated metrology, that can be standardized and used by industry.

Work on the first formability test, the springback cup, was undertaken at the direct request of industrial representatives in the United States Council for Automotive Research (USCAR). Springback is the shape change that occurs during elastic relaxation of the residual stresses that develop during the stamping process. Accurate springback predictions would greatly decrease the cost and number of iterations required for producing stamping dies.

The proposed test for springback consists of splitting open a ring cut from the sidewall of a deep drawn cup. The springback cup test was submitted to ASTM, and a subgroup has been formed to seek its standardization. In addition, an alternative springback standard based on stretch flanging over a cylindrical mandrel, developed by Kuwabara of Japan, has been submitted to ISO, with NIST staff developing the ASTM commentary on shortcomings of the standard geometry and procedures.

The second test method under development uses unique X-ray system that allows the direct, in situ measurement of stress in a given direction while the sample is under multiaxial load. This year we have made significant progress in mapping the multiaxial stress-strain surface for DQSK steel, an industry workhorse alloy, as well as high-strength steels such as HSLA and DP600. As with the aluminum alloys studied last year, both the flow stress and the hardening exponent were found to differ in the transverse and rolling directions of the sheet. These data will be used by industry to eliminate several assumptions that extrapolate estimated biaxial flow behavior based on a series of uniaxial tensile tests. This should lead to more accurate designs and cost savings.

This year, NIST was asked to generate materials property data for simulations of prototype parts as part of the NUMISHEET 2005 conference. In addition, the NUMISHEET 2005 benchmark tests are adding a new level of complexity by requiring modelers to predict stresses at specific sample positions during a benchmark forming process. NIST provided the corresponding measurements using our unique capabilities described above. This work also will be the subject of a plenary presentation at NUMISHEET 2005.

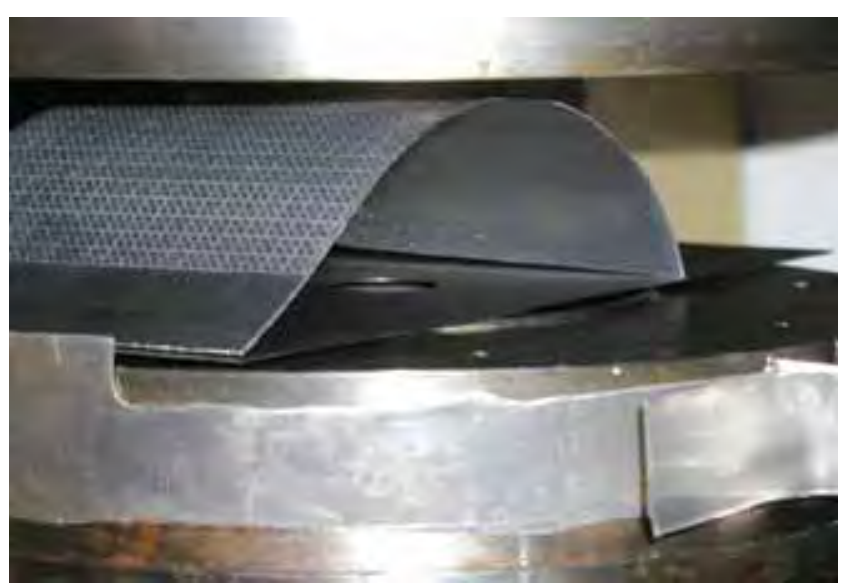

Figure 1: NUMISHEET benchmark sample loaded for plane strain deformation.

Plans for the upcoming year involve completing measurement of the multiaxial stress-strain surfaces for several alloys. This project will determine how the flow surface evolves with different types and amounts of multiaxial prestrain in a collaboration with modelers at GM. In addition, studies to date show that the influence of plastic strain on the elastic modulus of the material appears to be significant, and we intend to quantify the affect of plastic strain state and magnitude on the effective $x$-ray elastic constants of aluminum and alloy steel sheet.

\section{Contributors and Collaborators}

R.F. Fields; T. Gnaupel-Herold (NIST Center for Neutron Research); M. Shi (USS); E. Chu (ALCOA); C. Xia (Ford); T. Stoughton, M. Wenner, C.T. Wang (GM); J. Siekirk (DaimlerChrysler) 


\section{Microstructural Origins of Surface Roughening and Strain Localizations}

Existing data, measurements methods, and basic understanding of metallurgical factors that influence friction, tearing, and surface finish during sheet metal fabrication are insufficient to meet the predictive modeling requirements of the automotive industry. This project addresses the measurement and data needs through evaluation of the microstructural origins of the distribution of slip, surface roughening, and strain localization during plastic straining. These studies focus on the relationships between initial material characteristics and deformation behavior of aluminum and iron base sheet. New protocols have resulted that incorporate high-resolution confocal microscopy imaging to assess the roughening character over an entire field of view.

\section{Mark R. Stoudt and Stephen W. Banovic}

T ightweight materials, such as high-strength steels —and aluminum alloys, are designed to reduce vehicle weight and increase fuel economy. The widespread use of these new alloys in automotive components is limited, however, by insufficient property data and constitutive laws required for accurate numeric predictions of the mechanical behavior and friction during metal forming processes. One approach to meeting these critical data needs is a careful examination of the structure property relationships that directly influence formability.

The inability to reliably predict the deformed surface morphology with numeric methods raises serious questions as to how well the analytical tools employed to interpret roughness data actually represent the real surface. The underlying principles used to interpret surface roughness measurements were examined to ascertain the magnitude and source of any inconsistencies. The study assessed the variability within the surface roughness data that occurs over a surface by acquiring multiple roughness profiles from regularly spaced locations on an image (Figure 1) and establishing the actual form of the data with a set of mathematically rigorous protocols.

Most roughness analyses tacitly assume that a measured profile is completely described by ideal Gaussian statistics. However, the deviations observed in the skew and kurtosis data in this study revealed that roughness profiles taken from plastically deformed surfaces do not exhibit an ideal Gaussian form.

The significance of this conclusion is twofold:

first, non-Gaussian behavior means the roughness is produced by a small number of active mechanisms. This agrees with the literature in that deformation-induced surface roughness is primarily crystallographic slip and near grain boundary deformation. Second, these deviations from ideal Gaussian behavior provide direct evidence that the roughening behavior of a polycrystalline material cannot be accurately characterized with any parameter that presumes an ideal Gaussian form (i.e., mean( $\mathrm{Ra})$ or $\mathrm{rms}(\mathrm{Rq})$ ). These parameters also compress the complex surface information within a profile into a singular expression that can be quite coarse with respect to the length scale of the surface features.

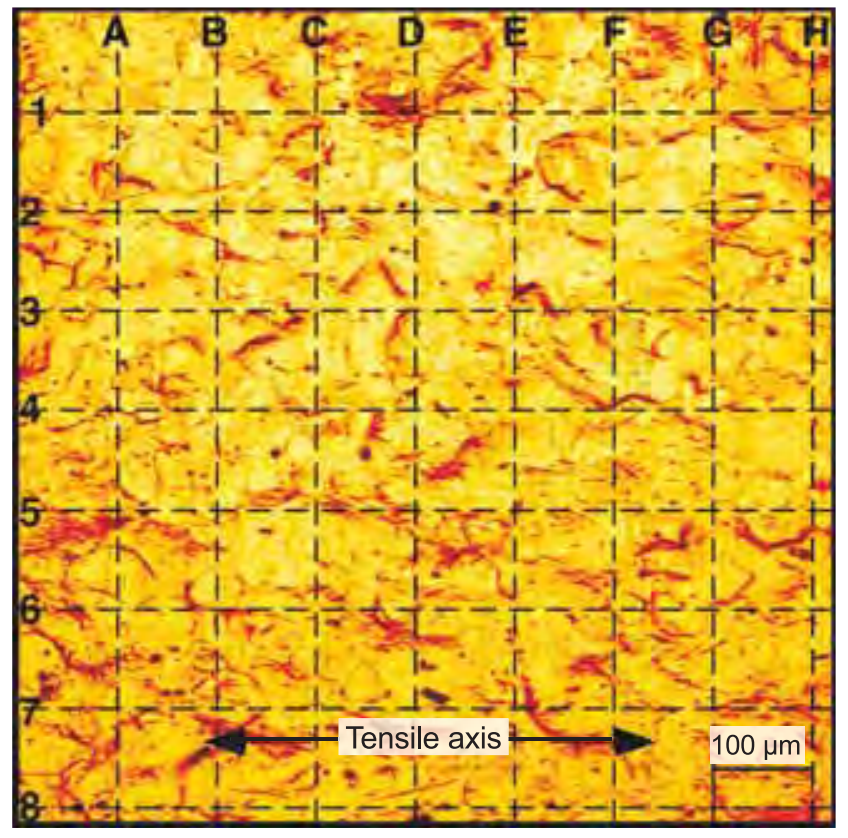

Figure 1: Scanning laser confocal image of the surface produced by $12 \%$ uniaxial strain in AA6022. The dashed lines indicate the general locations where surface roughness data were acquired.

Since surface roughness evaluations are highly dependent upon the statistical methods used in the analysis, small deviations from the ideal condition, such as those produced by plastic deformation, are likely to have pronounced influences on the accuracy of the analytical methods used to interpret the roughness data. Hence, the current practice of extrapolating averaged, compressed values into representations of an entire surface in a particular strain condition introduces substantial measurement error into numeric predictions of behavior. Expressions are being developed to more accurately represent the stochastic nature of variations in surface roughness.

\section{Contributors and Collaborators}

J.B. Hubbard, R.E. Ricker, L.E. Levine (Metallurgy Division, NIST); S.A. Janet (ITL, NIST); R. Reno, E. Moore (UMBC); J.T. Liu (ALCOA) 


\section{Underlying Processes of Plastic Deformation in Metal Alloys}

A substantial increase in the use of aluminum alloys and high-strength steels in automobiles would greatly increase fuel efficiency. The primary reason why this has not yet occurred is a lack of accurate deformation models for use in designing the stamping dies. This project is developing a physics-based model of plastic deformation using a combination of statistical physics approaches, atomistic modeling, and advanced measurement techniques.

\section{Lyle E. Levine}

$\mathrm{P}$ lastic deformation of metals (as in cold rolling, stamping, drawing, and metal fatigue) is a topic of great importance to industries worldwide, and improvements in the basic technology would have a significant impact on our economy. Unfortunately, existing constitutive equations cannot accurately predict the material behavior, and many tryout and redesign steps are required. Another related difficulty is in the design of new alloys with improved formability characteristics. Currently, alloy design is done empirically with little understanding of how the various constituents affect the mechanical properties.

In the current work, we have developed a theoretical framework called the segment length distribution (SLD) model for fcc single crystals that describes how macroscopic deformation arises from the statistical behavior of large numbers of dislocations. Although work on the SLD model is continuing, the primary emphasis over the past year has been on validation testing with Washington State University and new experimental measurements of the distribution of stresses within dislocation cells (with the University of Southern California and Oak Ridge National Laboratory). These stresses could affect the assumptions used in the SLD model.

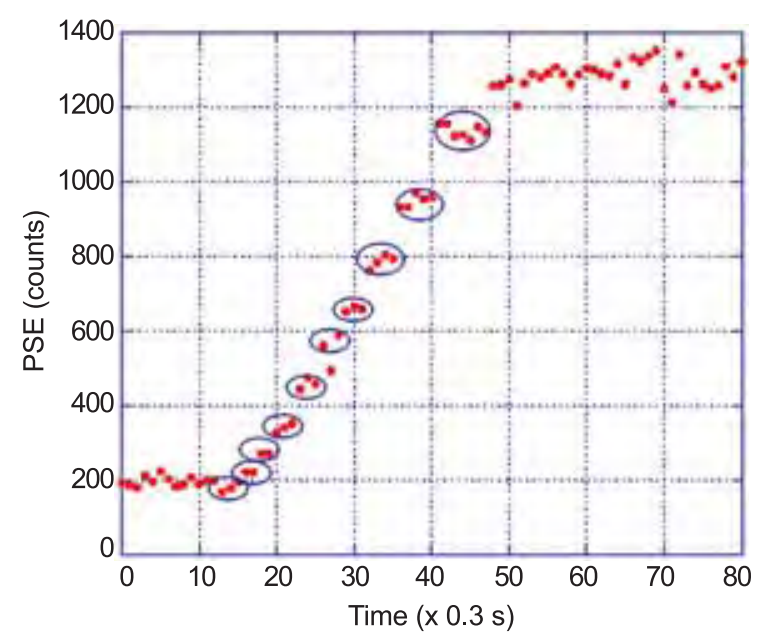

Figure 1: PSE data from Al single crystals.
Single crystal aluminum tensile specimens are being produced at NIST for the validation tests. These specimens are then strained, in situ, in ultra-high vacuum. A pulsed ultra-violet laser produces photostimulated electron (PSE) emission from fresh (unoxidized) $\mathrm{Al}$ in the slip lines/bands, and the electrons are collected by an electron multiplier. These time-resolved studies allow us to measure the time dependence of slip line/band production and compare these results to SLD model predictions. The SLD model predicts intermittent slip events under some conditions. Figure 1 shows preliminary PSE results that are consistent with these predictions.

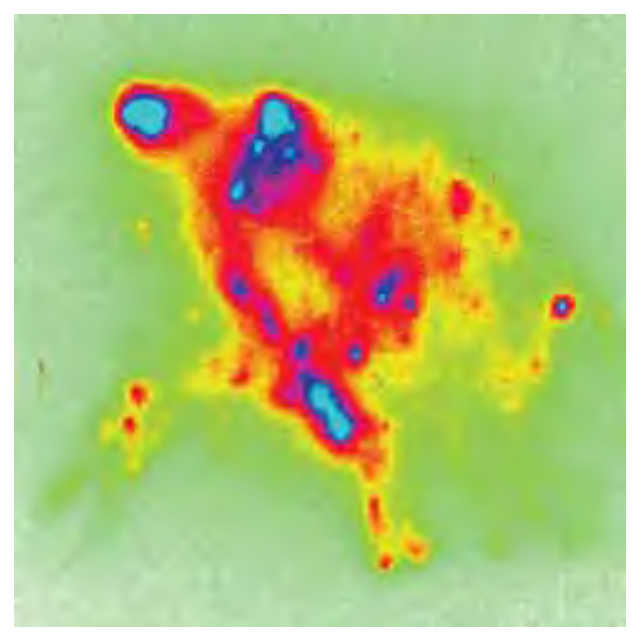

Figure 2: 117 reflection from deformed Cu crystal.

Preliminary measurements of residual stresses in plastically deformed $\mathrm{Cu}$ single crystals were carried out using X-ray micro-beams at the Advanced Photon Source, Argonne National Laboratory. Spatial resolution (in all three dimensions) is approximately $0.5 \mu \mathrm{m}$. Large fluctuations in elastic strain (and thus stress) were observed over length scales consistent with dislocation cells. Figure 2 shows an integrated energy scan of a 117 reflection from a Cu single crystal deformed $24 \%$ in compression. The localized and diffuse features originate primarily from dislocation cells and cell walls, respectively.

Finally, NIST researchers served on the executive committee of Dislocations 2004, and were co-organizers of Fall 2004 MRS and Plasticity 2005 symposia.

\section{Contributors and Collaborators}

F. Tavazza (Metallurgy Division, NIST);

A. Chaka (Physical and Chemical Properties Division); T. Dickinson, S. Langford, M. Cai (Washington State University); M. Delos-Reyes, Z. Gao, M. Kassner (University of Southern California); B. Larson, W. Yang (Oak Ridge National Laboratory) 


\section{Evaluation of Friction Behavior During Metal Forming}

Unpredictable friction resulting from

inhomogeneous surface deformation is a significant obstacle to the widespread introduction of new high-strength alloys in the automotive industry. Metal-forming computer models still rely heavily on empirical friction data. Most existing approaches are unable to address the strong influences that variations in metallurgical condition have on the dynamic material properties and on the evolution of surface roughness. This project addresses the need for improved friction measurements, data, and models, which will allow the auto industry to improve die design and processes for forming lightweight, high-strength sheet metal alloys.

\section{Mark R. Stoudt}

$\mathrm{M}$ any new alloys intended to decrease automobile weight are sensitive to variations in processing conditions. This variability causes inconsistencies in predicted friction behavior that exacerbate the forming difficulties. Traditional measurements normally focus on friction mechanics and do not account for the strong influences that dynamic loading and variations in metallurgical conditions have on the material properties. Hence, the friction values selected for a finite element analysis (FEA) simulation may not fully reflect the actual metal behavior under those particular conditions. Since one measurement cannot provide all essential data, new approaches are needed that: (a) evaluate both static and dynamic properties, and (b) improve the understanding of the relationships between friction and microstructural variations that affect properties during metal forming.

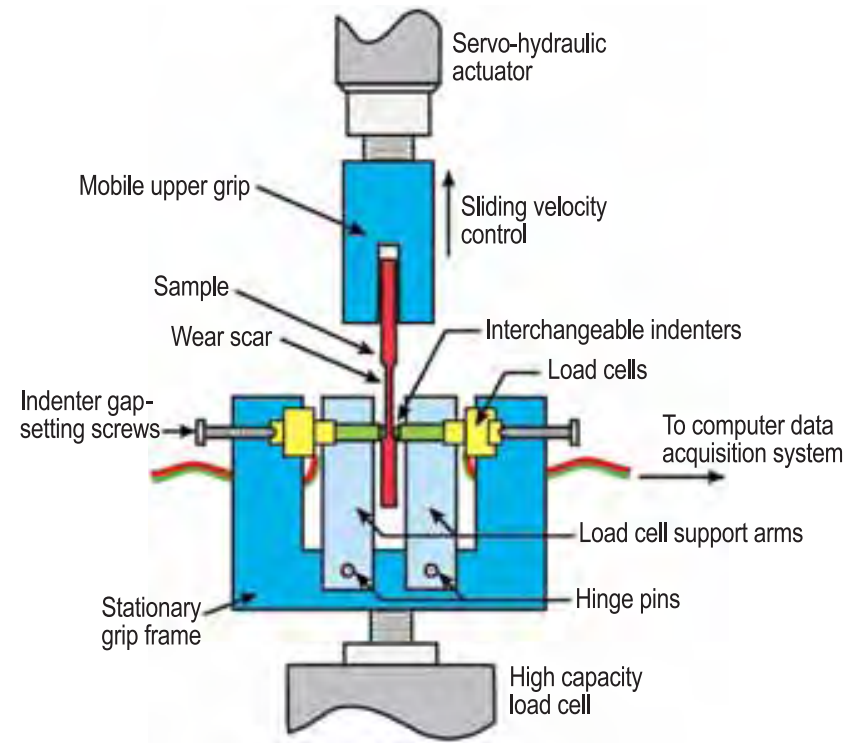

Figure 1: Schematic diagram of the prototype friction apparatus.
A single-pull measurement protocol (shown schematically in Figure 1) that produces friction data under a wide range of carefully controlled loading and strain conditions to help meet this critical need is under development. The principal advantage of this technique is that it enables direct assessment of the influences of metallurgical variables (i.e., grain size, orientation effects, strengthening mechanisms, slip homogeneity) as well as microstructural changes that are produced during deformation. This new information is anticipated to guide the development of improved numeric models that predict the final shape of formed parts.

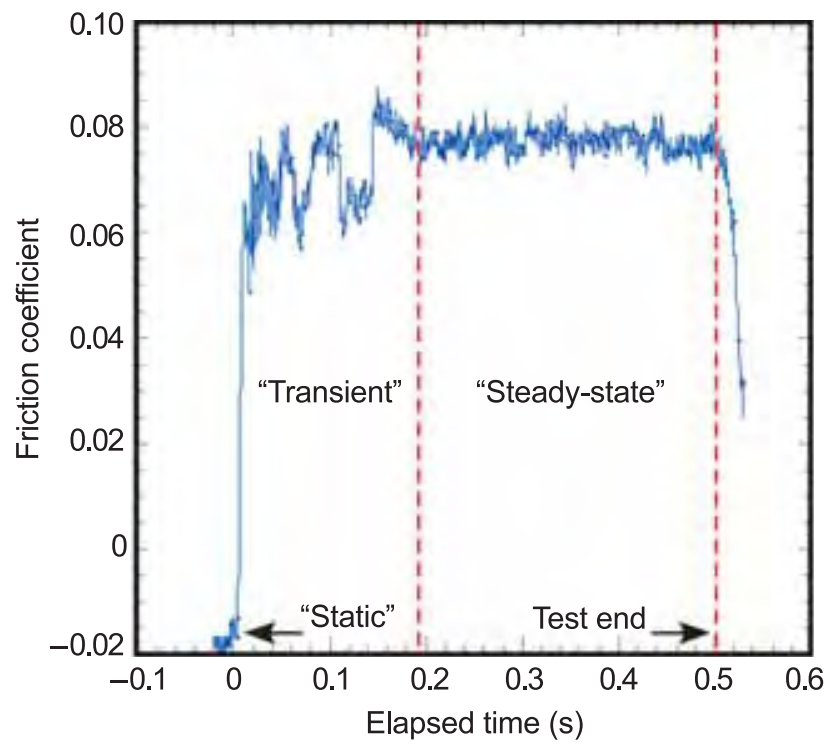

Figure 2: Variation in friction coefficient as a function of time for lubricated DQSK steel under a $1250 \mathrm{~N}$ indentation force.

The suitability of this approach was established through a series of friction experiments conducted on hot-dipped draw quality semi-killed (DQSK) steel. The data in Figure 2 demonstrate that the prototype can easily distinguish the transition from the static to the sliding state and that the magnitude of the friction coefficient can be determined in real time at any point during the test. In addition, the measurement sensitivity of the apparatus is such that the quality of the resulting data is amenable to detailed statistical analysis.

Results to date indicate that the measurement protocol is appropriate for further development. A matrix of experiments is planned to further evaluate the influences of initial surface roughness and asperity distribution, strain rates and surface lubricants.

\section{Contributors and Collaborators}

S.P. Mates, J.B. Hubbard, D.J. Pitchure, R.E. Ricker, L.E. Levine (Metallurgy Division, NIST); G.B. Dalton, (TribSys, Inc.); D.E. Green (University of Windsor) 


\section{Biomaterials}

Rapid development of medical technologies depends on the availability of adequate methods to characterize, standardize, control, and mass produce them. To realize this goal, a measurement infrastructure is needed to bridge the gap between the exponentially increasing basic biomedical knowledge and clinical applications. The MSEL Biomaterials Program is a collaborative effort creating a new generation of performance standards and predictive tools targeting the metrology chain for biomedical research.

Today, all areas of materials science confront real systems and processes. In the biomaterials arena, we can no longer advance science by simply studying ideal model systems. We must comprehend complex realistic systems in terms of their structure, function, and dynamics over the size range from nanometers to millimeters. MSEL is uniquely positioned to make a major contribution to the development of measurement infrastructure through three focus areas: Systems Biology, Bioimaging, and Nanobiosensing.

\section{Systems Biology}

MSEL research in systems biology focuses on quantifying relationships of systems at the cell, tissue, and organ level. To meet this need, we are developing libraries of reference materials, high-throughput techniques for screening libraries, and informatics approaches for data analysis and interpretation. Physicochemical and biochemical components are organized using patterning, phase separating, and self-assembling processes. Physicochemical components of interest include modulus and surface topography; biochemical components of interest include peptide moieties that interact specifically with cell receptors.

Gradient libraries of tyrosine derivatized polycarbonate blends and fibronectin/poly(hydroethylmethacrylate) gradients were developed as reference materials for biomaterial research, such that cell responses included changes in geometry, distribution, and proliferation, to assess intercellular communication among osteoblast and fibroblast cells. Complementing these surface studies, we are developing metrologies to establish the relationship between 3D scaffold morphology (i.e., porosity and permeability) and cell response. Studies focused on identifying the relationship between applied macroscopic stresses and local stresses at the cellular level is also underway, which will provide valuable input into development of finite-element models.
Experiments on the mechanical stimulation of tissues and tissue engineered constructs were conducted to understand the role of metrology in diagnostic testing of healthy or disease states. Stress-strain relationships were defined for vascular smooth muscle cells and bovine cardiac tissues. Specialized bioreactors coupled to ultrasound and infra-red spectroscopy were successful in differentiating response among the systems. We have demonstrated that the structure-property relations in healthy tissue of pulmonary arteries, and in tissue that has remodeled in response to the onset of disease, can be assessed using mechanical testing, quantitative ultrasonic characterization, and histology.

\section{Bioimaging}

Advances were made in developing and optimizing physical methods and informatics tools to enhance bioimaging and visualization technologies at multiple length scales. With the reduction of background noise, images were obtained using broadband coherent anti-stokes Raman scattering microscopy with a 10 -fold increase in signal, and proteins on the surface of polymer blends were differentiated. Optical techniques like OCM and CFM, with spatial resolutions of $\approx 1 \mu \mathrm{m}$, were employed to image dynamic cell culture experiments in-situ in a bioreactor. Other advances in computational modeling of single cell forces and cell populations were carried out to predict normal ossification patterns and cartilage formation. By combining information from different techniques on the same sample and visualizing structure using interactive, immersive visualization techniques, scientists will gain new insights into the physics and materials science of complex systems.

\section{Nanobiosensing}

Research in this focus area concentrates on the development of techniques to measure and manipulate biological atoms, molecules, and macromolecules at the nanoscale level (1-100 nm). Mechanical tools including an optical trap and bioMEMS devices that can be integrated with currently used biological techniques for evaluating and measuring cellular response (i.e., gene expression, cell morphology, area of adhesion) were developed. Additional studies focus on identifying mechanical forces that indicate the onset of osteogenesis and angiogenesis.

Contact: Lori A. Henderson (Polymers Division) 


\section{Combinatorial Methods for Rapid Characterization of Cell-Surface Interactions}

\begin{abstract}
The increasingly complex nature of functional biomaterials demands a multidisciplinary approach to identify and develop strategies to both characterize and control cell-material interactions. A robust framework outlining the interactions governing biomaterial performance does not exist but is desperately needed. This project provides the basis for this framework by focusing on fabrication of single and multi-variable continuous combinatorial libraries to rapidly identify compositions and physical properties exhibiting favorable cell-material interactions.
\end{abstract}

\section{Matthew L. Becker and Lori A. Henderson}

$\mathrm{D}$ evelopmental biology and tissue engineering are avenues of research that must be fully integrated to realize the opportunities in regenerative medicine. For example, while the interactions between cell and extracellular matrix have been studied extensively, much less is understood regarding the influence of synthetic materials. There is little doubt that having good control of surface morphology as well as advanced high-throughput (HT) metrologies for analyzing cell-surface interactions are needed for biological interpretations, and while chemical and topographical manipulations of surfaces have been established, HT methods to evaluate biological responses to these manipulations have not. For these reasons, we are developing metrologies and HT platforms to rapidly analyze physicochemical, mechanical, and material properties of biomaterials. We provide examples of two of our sample fabrication methods, distinct from traditional self-assembled monolayer approaches, that are being used to design, manipulate, and quantify cell-surface interactions.

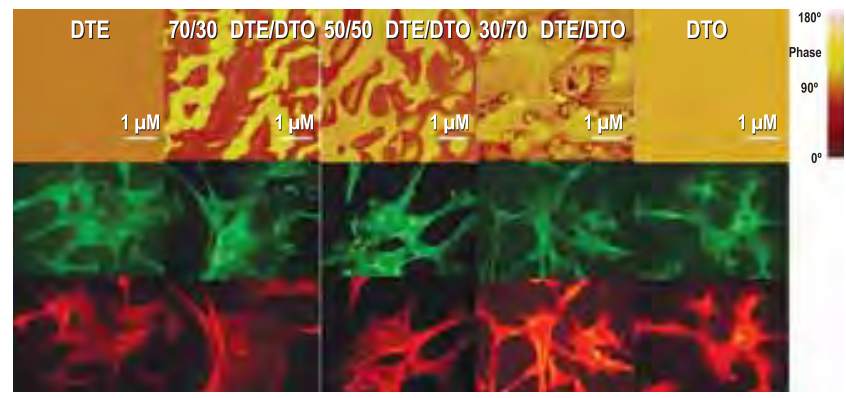

Figure 1: AFM micrographs of the tyrosine-derived polycarbonate homopolymers and discrete blends show compositionally dependent phase separation, which is reflected in the immuno-fluorescent staining for actin (red, cell spreading) and vinculin (green, focal adhesion contacts) on MC3T3-E1 osteoblasts.
Two functional polymer surfaces, phase separated tyrosine-derived polycarbonate blends (DTR-PC) and conformational-based poly(2-hydroxyethyl methacrylate) brushes [poly(HEMA)], were analyzed using combinatorial methodologies. The DTR-PC films, consisting of homopolymer and discrete composition blends of tyrosine-derived polycarbonates, were shown to have compositionally dependent gene expression profiles with the blends differing significantly from the respective homopolymers. Figure 1 illustrates the effect that polymer blending has on cell spreading; the extension and distortion of the lamellapodia increase and the cells appear to spread less in the blend samples with increasing DTO content. The surface properties from these discrete films will be used to establish correlations and limitations for comparing measurements from discrete samples and single and multi-variable continuous gradient substrates.

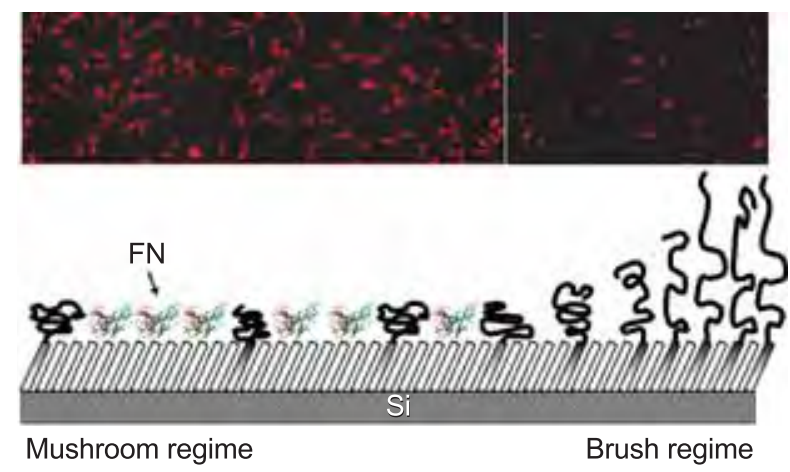

Figure 2: Schematic representation of the poly(HEMA)-FN gradient and Fibroblast cell distribution.

Poly(HEMA) gradients were prepared to study molecular interactions and cell conformation on fibronectin (FN) coated poly(HEMA) by combining "controlled" free radical polymerization with gradient preparation technology. This gradient covers "mushroom" to "brush" regimes in order to determine how grafting density influences protein adsorption and cellular response as shown in Figure 2. The number of cells, their shape, and size were thus correlated to the density of fibronectin across the gradient.

In summary, the tools developed in this program will enable the design of material libraries to be used to probe the behaviors of cells.

\section{Contributors and Collaborators}

N.D. Gallant, L.O. Bailey, C. Simon, Jr., T.W. Kee, Y. Mei, J.S. Stephens, E.J. Amis (Polymers Division, NIST); K. Langenbach, J.T. Elliot (Biotechnology Division, NIST); J. Kohn, A. Rege, J. Schutt (Rutgers University \& The New Jersey Center for Biomaterials) 


\section{Cellular Level Measurements}

Techniques and tools that facilitate the exposure of single cells (and arrays) to controlled mechanical environments and quantification of mechanical forces, and at the same time allow for the characterization of other biological phenomena, are needed for the study of tissues and cells. The development and evaluation of one of these tools, a Bio-MEMS cell puller, is the focus of this year's effort.

\section{Andrew J. Slifka}

$\mathrm{R}^{\mathrm{e}}$ esearch on the mechanical response of biological materials at the cellular and sub-cellular levels is being done with the development of a number of tools. Optical trapping for cellular mechanics and small-force materials science is one. We use an optical trap with a scanned laser to trap multiple dielectric spheres. These spheres can be attached to a cell. We plan to use four balls to perform a biaxial mechanical measurement of a single cell. We are developing data analysis routines to allow measurement of transient mechanical response. The optical trap is also being used to study attractive forces by measuring those forces required to pull apart two-dimensional islands of self-assembling nanoparticles.

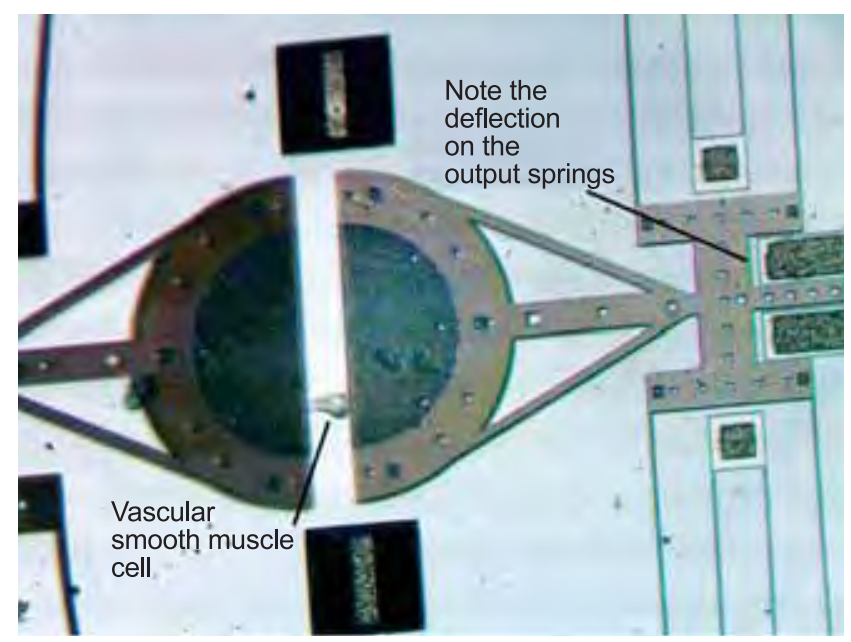

Figure 1: Detail of the cell platform of the Bio-MEMS device used for mechanical and adhesion measurements, showing a cell being stretched.
Another tool uses Bio-MEMS for cell pulling and adhesion. Both adhesion and the mechanical response of a cell to varying mechanical environments are fundamental to understanding cell motility and numerous disease mechanisms. A device has been designed and built that has a transparent platform on which a single cell can adhere. The focal adhesions of the cell can be viewed using reflection interference microscopy or appropriate staining. The platform is split so that the cell can be strained from one side and forces measured on the other by way of thin cantilever beams (see Figure 1). Image analysis can yield strain and also the cross-sectional size of the cell so that stress can be determined using the force information. Therefore, force- or stress-strain response of a single cell can be done in addition to force as a function of adhesion area. The device and instrumentation can also be operated in a cyclic mode, which can be used to determine the change in mechanical response of the cell as a function of cyclic fatigue. The design of this device and results on vascular smooth muscle cells will be presented at the Biomedical Engineering Society Annual Fall Meeting in Baltimore, Maryland in September.

Another tool under development is a Bio-MEMS device for quartz-crystal microbalance (QCM)-type measurements. The QCM is used extensively in the medical research field for characterizing antibody systems. It is a macroscale device with high sensitivity due to its oscillating nature. We believe that a Bio-MEMS device that works similarly could yield a thousand-fold increase in sensitivity and have as wide application as the QCM. We have made measurements on a model antibody system as a baseline and have designed and built a Bio-MEMS device that mimics the QCM. We have developed a model of the device response in order to optimize future designs for particular applications. A manuscript on the device design and model has been written for the Journal of Applied Physics. We are pursuing simple methods for electrical measurement of the change in oscillator response, which is the heart of the QCM measurement technique.

\section{Contributors and Collaborators}

V.M. Aponte, E.S. Drexler, D.S. Finch, D. Lauria, C.N. McCowan, H. Panchawaugh, R.A.L. Rorrer, D.B. Serrell, R.P. Vinci (Materials Reliability Division, NIST) 


\section{Cell Response to Tissue Scaffold Morphology}

Industrial and regulatory sectors have expressed a need for standards and new metrologies relating to properties of tissue scaffolds for regenerative medicine. We seek to meet these needs in several areas where the criteria are clear, and to help clarify industrial and regulatory needs in other areas where such clarification is required. We are developing a reference scaffold for porosity and permeability. Also, we are developing metrologies for establishing the relationship between scaffold porosity/morphology and cell response, for assessing the ability of a tissue scaffold to safely host cytokine, and for quantifying mechanical stimulation requirements for cells - at the cellular level - from macroscopic inputs.

\section{Marcus T. Cicerone}

Tn the field of regenerative medicine, one seeks to Iguide cell differentiation and proliferation, and production of the extracellular matrix through functional properties of 3D tissue scaffolds. Developing the ability to guide such cell behaviors requires first the ability to characterize and assess properties of tissue scaffolds as they relate to cell response. This, in turn, requires well-defined physical and biological systems for which quantitative rules can be formulated and verified.

We are developing methods for quantitatively characterizing tissue scaffolds and the cellular responses they elicit. There are three classes of scaffold properties that we focus on relative to their impact on cell behavior; these are: (i) morphological/ topological properties, (ii) mechanical properties, and (iii) ability of biodegradable scaffold materials to act as biopreservents in connection with hosting growth factors and other cytokines.

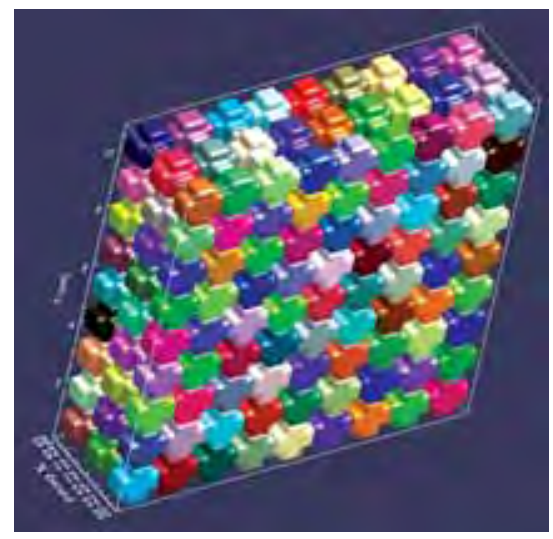

Figure 1: A reconstruction of a candidate reference scaffold, generated from a tomographic image. Each colored object represents a separate unit cell within the pore structure of the scaffold.
We led a worldwide collaboration under ASTM with 17 other laboratories to establish a series of reference scaffolds for porosity and permeability. Our primary characterization method for these scaffolds is based on tomographic image analysis of scaffold morphology.

We are developing metrologies for assessing osteoblast response to pore size distributions in tissue scaffolds based on extracellular matrix (ECM) production. We are also investigating morphology effects on osteoblast response to surface chemistry. The image analysis methods developed in the reference scaffold activity serve to support these efforts. The ability to uniformly and reproducibly seed 3D scaffolds with adherent cells is another critical factor for quantifying links between scaffold morphology and cell response, and we have developed methods to accomplish this.

We are using computational modeling coupled with high-resolution imaging, atomic force microscopy (AFM), and optical trapping to develop metrology in the area of cell response to environmental mechanical stresses. It is clear that mechanical stimulation is required for some cell types to differentiate properly. Thus, it is important to be able to measure precisely what stress conditions are necessary for proper phenotypic expression for selected cell types. We are collaborating with the Materials Reliability Division of MSEL to establish methods to quantify the stress conditions at the cellular level based on macroscopic forces placed on the scaffold construct. Our approach is to translate ranges of macroscopic stresses to local stresses experienced by cells using a finite element model. These local stresses will be correlated with cell response in terms of ECM production.

Biopreservation of cytokines in tissue scaffolds is a complex but important area of regenerative medicine that has been historically underserved. We are collaborating with six academic and one national lab to create a holistic approach to stabilizing proteins in solid hosts such as tissue scaffolds. We are leading the grant-writing efforts in this collaboration and are focusing on clarifying the relationship between fast glassy dynamics and biopreserving ability of a material, which we have already observed in neutron scattering experiments. In keeping with this goal, we are establishing accessible time-resolved optical metrologies for measuring these dynamics.

\section{NIST Contributors and Collaborators}

J. Dunkers, F. Wang, J. Cooper, T. DuttaRoy, J. Stephens, F. Phelan, M.Y.M. Chiang, L. Henderson (Polymers Division, NIST); Tim Quinn (Materials Reliability Division, NIST) 


\section{3-Dimensional In Situ Imaging for Tissue Engineering: Exploring Cell/Scaffold Interaction in Real Time}

Real time investigations of cell/scaffold interactions provide valuable information about the dynamic nature of cells and their spatial arrangements with respect to the three-dimensional (3D) architecture of tissue engineering scaffolds. In situ imaging capabilities will enable determination of the structure/function relationship of tissue engineering scaffolds and definition of the necessary properties to promote tissue regeneration. We demonstrate tools for in situ imaging of cells/scaffold interactions.

\section{Jean S. Stephens and Joy P. Dunkers}

$\mathrm{T}_{\mathrm{i}}^{\mathrm{h}}$ he ability to image live cells and their corresponding interactions with the surrounding environment provides critical information about the ability to promote desired cellular activity (proliferation, differentiation, etc.) for tissue regeneration. In order to develop in situ optical imaging capabilities, we must be able to nondestructively and noninvasively image the interactions at the cell/scaffold interface while maintaining cell viability.

In our laboratory, collinear optical coherent microscopy/confocal fluorescence microscopy (OCM/CFM) has successfully been used to image the 3D interconnected porous structure of polymeric scaffolds. This system combines high spatial resolution ( $\sim 1 \mu \mathrm{m})$, high sensitivity (>100 dB), and exceptional depth-of-penetration associated with OCM with the
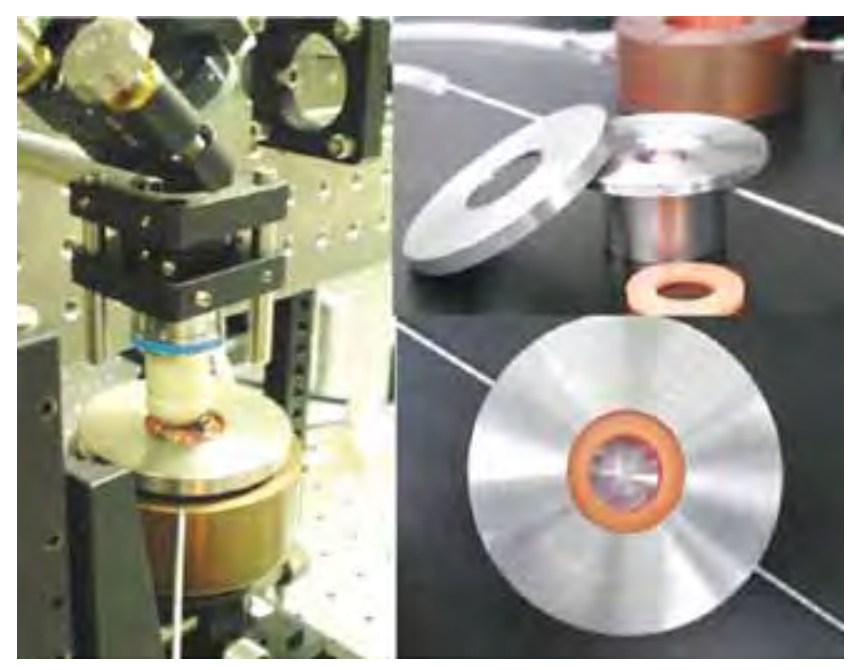

Figure 1: Bioreactor in OCM/CFM, open and top view. fluorescent capabilities of CFM. This, therefore, allows us to not only investigate the 3D scaffold, but also the use of conventional fluorescent staining techniques to evaluate cellular response.

In order to perform live cell imaging, a system or bioreactor that can sustain cell viability outside of an incubator and allow for imaging was constructed (Figure 1). The bioreactor is a perfusion flow bioreactor. This design forces the media to flow through the scaffold, therefore ensuring nutrient delivery and oxygen perfusion, as well as waste removal, throughout the entire structure. Also, a dynamic cell culture creates an environment that better mimics physiological conditions. The temperature of the bioreactor system is maintained by circulating water $\left(37^{\circ} \mathrm{C}\right)$ through a copper element.

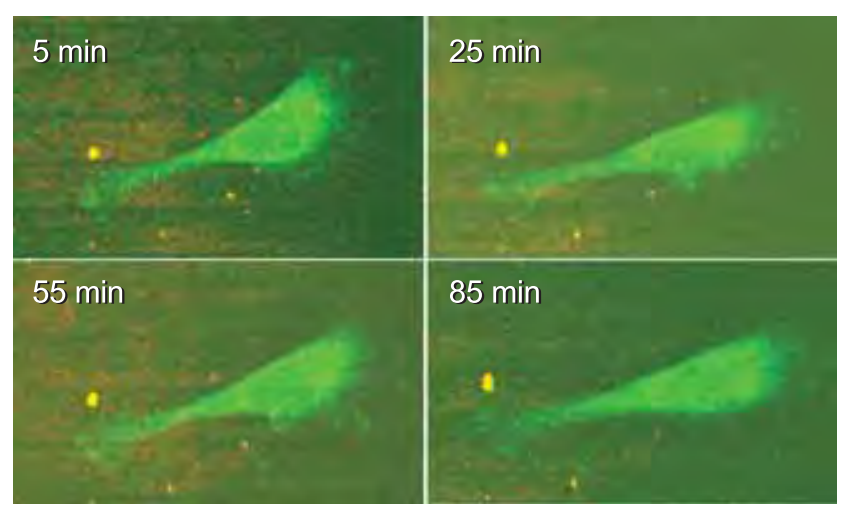

Figure 2: Time-lapse images of cell movement.

Initial in situ imaging studies indicate the maintenance of cell viability, and we have successfully imaged cells for several hours. The series of images in Figure 2 illustrate cell movement over a 2 hour time period. The ability to collect images in real time will give a great insight and understanding of how cells are responding to different materials, scaffold architectures, and culture conditions. These data will provide new metrics for the evaluation of tissue engineering scaffolds.

\section{Contributors and Collaborators} NIST) 


\section{Broadband CARS Microscopy for Cellular/Tissue Imaging}

In many of the biological sciences, as well as many areas of polymer science, there is a need for high-resolution, noninvasive, and chemically sensitive imaging. We have developed a broadband coherent anti-Stokes Raman scattering (CARS) microscopy that provides an unprecedented combination of imaging speed and spectral coverage (i.e., chemical sensitivity). Our current efforts are focused on eliminating nonresonant background effects, which can limit sensitivity of the technique.

\section{Marcus T. Cicerone}

$\mathrm{W}$ e have developed a broadband CARS microscopy method which allows us to obtain vibrational spectra in the range (500 to 3000 ) $\mathrm{cm}^{-1}$ in less than 1/50th the time required to obtain similar spectra by spontaneous Raman. This development was reported at the first meeting of National Institute for Biomedical Imaging and Bioengineering (NIBIB) grantees, in Bethesda, Maryland, the 11th Annual Time Resolved Vibrational Spectroscopy Conference, and the 2005 Biophysical Meeting.

One key to the method we have developed is the generation of a broadband continuum. Optical pumping of a tapered silica fiber was used to generate broadband continuum in the first prototype of this instrument. Accumulative photo-damage limits the lifetime of the tapered fiber, and seriously limits the power level of the light that can be generated, significantly restricting the taper fiber as a reliable light source for CARS microscopy. With the assistance of an outside vendor, we have designed and procured a photonic crystal fiber (PCF) that is sealed at the ends, and which avoids the above issues. The PCF did not show any sign of degradation after a month, under long-term irradiation of $40 \mathrm{~kW}$ peak power femtosecond laser pulses. This advance provided $\approx 10$-fold increase in signal levels, so that, in principle, we can gather broadband spectra in 1/500th the time required for spontaneous Raman spectroscopy. In practice, this rate exceeds the capabilities of the CCD camera, which therefore sets the limits on data acquisition; a faster camera would allow higher data collection rates.

A blend of chemically similar biodegradable polymers, abbreviated as DTE and DTO (see Figure 1a), have induced remarkable low immune response upon fibroblast cell adhesion. These two polymers phase-separate upon annealing, and since they have similar indices of refraction, optical microscopy cannot be used to image the phase-separated domains. On the other hand, broadband CARS microscopy has the sensitivity to distinguish the two polymers. Figure 1 shows the three-dimensional imaging of a 50/50 (a)
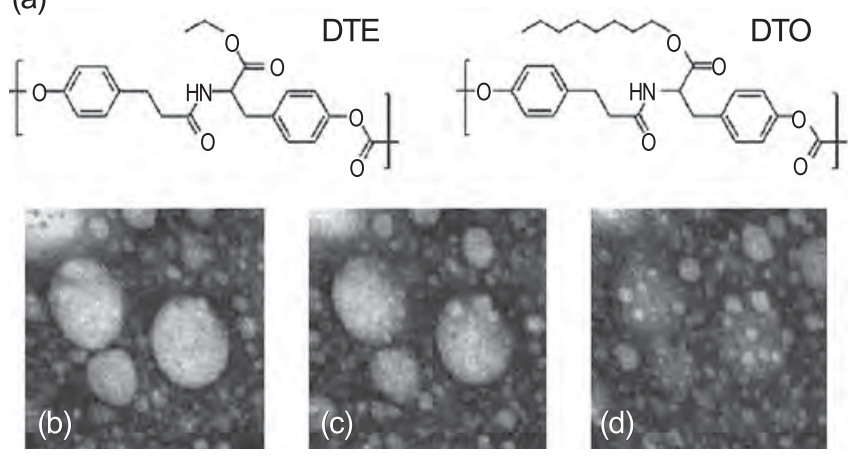

Figure 1: (a) Chemical structures of DTE and DTO. CARS images of a 50/50 DTE/DTO blend at depth: (b) $0 \mu \mathrm{m}$, (c) $3 \mu \mathrm{m}$, (d) $6 \mu \mathrm{m}$. The white areas in these images are DTO; the black regions are DTE.

DTE/DTO blend sample. In this sample, the spatial resolution is approximately $0.4 \mu \mathrm{m}$. We are currently exploring the hypothesis that the low cellular immune response to the blends has its origins in spatial patterning of the adhesion proteins. We are working to correlate the protein adsorption with spatial patterning of DTO and DTE rich domains.

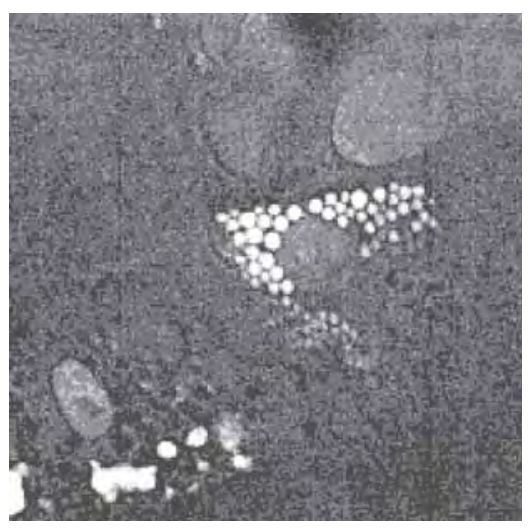

Figure 2: Micrograph of adipocyte. This image was obtained without the use of contrast agents such as fluorescent stains; the $2845 \mathrm{~cm}^{-1}$ $\mathrm{C}-\mathrm{H}$ stretch vibrational band was the only image contrast.

In Figure 2, bright circular features are triglyceride lipid droplets in adipocytes, and the more subdued quasi-circular objects are the cells. We were unable to image the presence of protein in the cytosol due to nonresonant background. Detection of these proteins is crucial to identifying cell type, and we are currently focusing our efforts on substantially reducing the effects of nonresonant background.

\section{Contributors and Collaborators}

T.W. Kee, H. Zhao, J. Taboas (Polymers Division, NIST); W-J. Li, R. Tuan (NIH/NIAMS) 


\section{Response of Tissues and Tissue-Engineered Constructs to Mechanical Stimulation}

\begin{abstract}
Mechanical stimulation of tissue as it is being grown in a bioreactor has been shown to make tissue engineered constructs more like healthy, natural tissue. However, little effort has gone into studying exactly how the stimulation should be done.[1] Mechanical stimulation can also be used for enhanced drug delivery. Researchers at the National Institutes of Health have been developing an ultrasonic system to enhance drug delivery in tumors that are not easily treated with chemotherapy. We are developing instrumentation and models to optimize these methods.
\end{abstract}

\section{Timothy P. Quinn, Tammy L. Oreskovic, and Brian E. O'Neill}

\section{Mechanical Stimulation of Tissue-Engineered Constructs}

\begin{abstract}
$\Lambda$ bioreactor has been developed that can provide Aboth mechanical stimulation to a tissue-engineered (TE) construct and mechanical testing while the tissue is being grown in the bioreactor. The reactor was designed to facilitate studies to determine the optimal variables for growing TE constructs for vascular grafts. In this bioreactor, we can stimulate a planar sheet of tissue/scaffold construct with an arbitrary waveform. The reactor can be configured to apply a given force or a given displacement. It is equipped with actuators, load cells, and viewports to conduct online, biaxial stress-strain tests without removing the sample from the reactor.
\end{abstract}

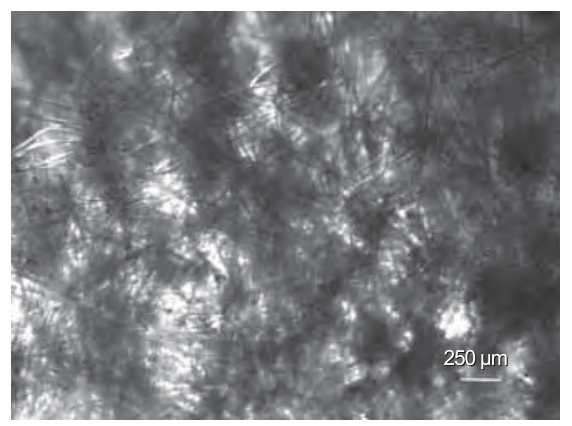

Figure 1: The biodegradable scaffold with tissue ingrowth taken during a stress-strain test. Image correlation is used to measure strain.

Human coronary smooth muscle cells (passages 7-9) were obtained from a commercial source and used in the experiments. The coronary smooth muscle cells propagated on the scaffold and were seen without difficulty by day 7 (Figure 1). No evidence of contamination was noted in the reactor at any time in the verification testing. Stress-strain testing was also accomplished during the trials. An example of the utility of online monitoring was evidence of damage to the scaffold in the form of fiber de-adhesion at a strain of $10 \%$.

\section{Mechanisms for the Increased Bioavailability of Materials Using High-Intensity Focused Ultrasound}

At low powers, the mechanical stimulation of high-intensity focused ultrasound (HIFU) can be used to increase the permeability of tissues and, therefore, could be used to increase the effectiveness of drugs delivered to treated tissues. At these power levels, the tissue is not permanently damaged but can heal and return to its original pre-HIFU state.

In order to optimize the HIFU treatment, we need to know the mechanism by which the tissue is made more permeable. To understand this, a simple model of the sound propagation is being developed together with a parameterized model of the openings ("cracks") between cells, and between cells and the extracellular matrix. Concepts from the field of damage mechanics (usually reserved for materials like metals) are being used to develop this model.

Supporting experiments using bovine cardiac tissue are being conducted to identify the unknown parameters to the models and to identify the mechanical mechanism that leads to the increased permeability. This newly implemented system relies on confocal therapeutic and imaging ultrasonic elements, packaged together commercially. A gated function generator and power amplifier is used to drive the outer, therapeutic HIFU element in pulsed mode at $1 \mathrm{MHz}$. An ultrasonic pulser/receiver simultaneously drives the inner $10 \mathrm{MHz}$ imaging element and collects data for processing. The system has a number of unique capabilities for biological application. The imaging element is used to monitor the radiation force, which has been proposed as the main mechanism behind the effect. Following designs used in the literature, we have constructed a device to measure the permeability of the tissue samples.

1. Martin, et al., Ann. Biomed. Eng., 27, 656, 1999.

\section{Contributors and Collaborators}

A. Slifka, E. Drexler (Materials Reliability Division, NIST); F. Landis, L. Henderson (Polymers Division, NIST); V. Frenkel, K. Li (NIH) 


\section{Mechanical Behavior of Tissue}

\begin{abstract}
Measurements of the structure and mechanical properties of biological materials elucidate mechanisms of disease, and permit quality assessment of tissue-engineered constructs. Certain diseases may be identified by changes in the mechanical properties of the affected tissue before loss of function is detected, thus enabling earlier diagnoses and intervention. Our objective is to provide support to clinicians by developing measurement techniques and by measuring the properties of tissue.
\end{abstract}

\section{Elizabeth S. Drexler}

$\mathrm{R}^{\mathrm{e}}$ esearch on the mechanical properties at the tissue level has focused on the properties of pulmonary arteries with the onset of pulmonary hypertension (PHT). The onset of PHT is known to cause remodeling of the walls of the pulmonary arterial system, and, if left untreated, can lead to right-side heart failure. We have tested the left and right main arteries, and the trunk (the arteries most accessible for clinical diagnostics) in the direction of blood flow and in the circumferential orientations. Our goals are: (i) to determine the onset of pulmonary hypertension by correlating our measured mechanical properties to the output of tissue Doppler and ultrasound techniques currently being developed as diagnostic tools and (ii) to develop structure-property relations.

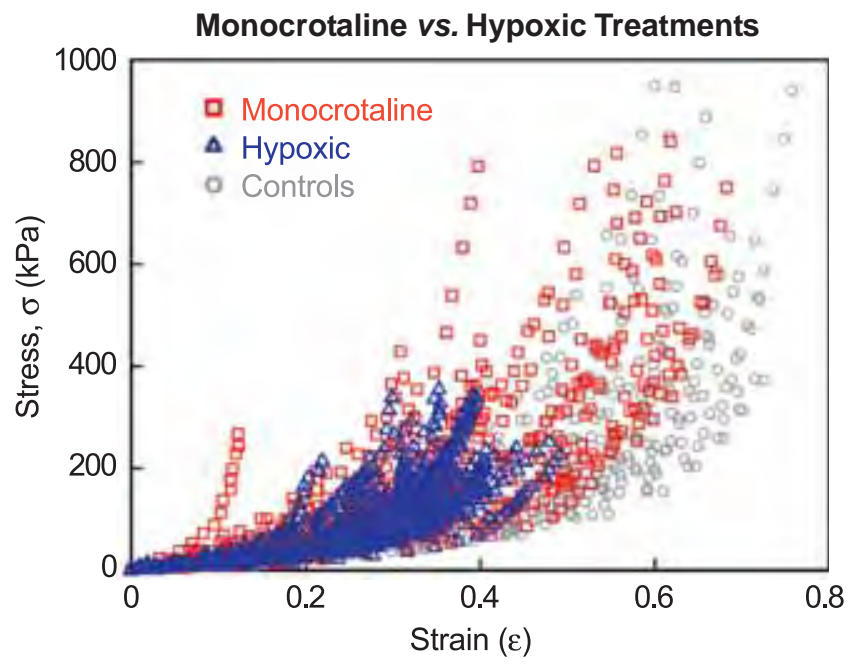

Figure 1: Graph showing the stress-strain behavior of the control, hypoxic, and monocrotaline-treated rat pulmonary arteries.

Using a rat model as a first approximation to the behavior of human arteries, we have measured the stress-strain properties of pulmonary arteries from four different populations: control, hypoxia-treated, mono- crotaline-treated, and hypoxia-treated genetically modified to disable a receptor responsible for activating vasodilators. Figure 1 shows the stress-strain behavior of the treated populations compared with the controls. The difference between the two hypoxic populations and the controls are readily obvious. However, the monocrotaline-treated data indicate that the arteries have not remodeled similarly to the hypoxic rats, and an entirely different mechanism, other that arterial wall remodeling, may be operating.

Complementary work is underway on the histology and quantitative ultrasonic properties of the pulmonary arteries. The reduction in elastic fiber, which accompanies the increase in thickness in the hypoxic samples is consistent with the change in mechanical properties typically associated with hypertension, where the arteries become less compliant. The increased thickness of the medial layer is mostly due to thicker muscular layers between elastic lamellae. At constant pressures, increases in thickness and reductions in elastin content contribute to a stiffer response. We have used quantitative ultrasonic characterization to correlate mechanical properties due to remodeling to the ultrasonic properties of the unstressed remodeled tissue. Ultrasonics is also used to predict fracture risk in osteoporitic patients. Dispersion (or how the speed of sound changes with frequency) appears to be sensitive to changes in bone mineral density. We organized a topical meeting on ultrasonic characterization of trabecular and cortical bone that was sponsored by the Acoustical Society of America.

The results comparing the arteries from the hypoxia-treated rats to those of the controls, along with this histology, were presented at the ASME Summer Bioengineering Conference in Vail, Colorado, in June. The comparison between the monocrotaline and hypoxic to the controls will be presented at the Biomedical Engineering Society Annual Fall Meeting in Baltimore, Maryland, in September. A manuscript on the technique, and the control results, was submitted to the Journal of Biomechanics. Three papers have been published on the topic of improving quantitative ultrasonics for tissue characterization, and a NIST IR is now available summarizing the Inaugural Workshop on Computational Tools for Modeling Acoustic Propagation in Real-World Materials. Additional presentations have been made at the IEEE International Ultrasonics Symposium in 2004 and the 148th Meeting of the Acoustical Society of America.

\section{Contributors and Collaborators}

C. McCowan, K. Waters, A. Slifka, T. Quinn (Materials Reliability Division, NIST); R. Shandas, D. Ivy, C. Cool, K. Colvin (University of Colorado Health Sciences Center) 


\section{Materials Design for Biomechanical Structures}

Biomechanical prostheses such as dental crowns, total hip and knee replacements, heart valves, and spinal disk devices are becoming more commonplace in an ever-aging population. The lifetimes of such prostheses are limited by materials properties. Accordingly, it is imperative that we understand the modes of failure in these systems, in order that materials can be developed for superior performance. This program with extramural partners (New York University, University of Maryland) and international institutions seeks to determine fundamental ground rules for designing biomechanical systems for improved lifetime performance by identifying and analyzing clinically relevant damage modes.

\section{Brian Lawn}

racking and other damage modes in ceramic layers because of the potential for lifetime-limiting premature failures. This is especially true of biomechanical prostheses - dental crowns, hip and knee replacements, heart valves, spinal disk replacements - where ceramic components introduced to enhance wear resistance, strength, chemical durability and, in the case of dental crowns, aesthetics are exposed to cyclic concentrated loads under stringent in vivo environmental conditions. A proper understanding of the materials aspects of any such ceramic-based prosthetic device becomes a quality-of-life issue. In many such applications the ceramic is just one component in combination with polymer and/or metal support sublayers, so a systems approach is essential.

In this program, we characterize contact-induced damage modes in model layer systems — bilayers, trilayers, and even multilayers - that simulate the basic loading features of biomechanical structures and, at the same time, allow direct in situ observations of the damage evolution during loading and unloading. The most revealing are model layer structures made from transparent components, e.g., ceramic coating layers on clear polymer substrates, or glass coatings on metal substrates. Critical conditions for damage initiation can then be directly monitored and quantified.

Using this approach, we have been able to identify damage modes believed to be responsible for the failure of clinical prostheses, especially dental crowns. Analytical relations expressing the critical applied loads (e.g., biting force, body weight on hip replacements) in terms of ceramic layer thicknesses and material properties (strength, toughness, modulus, hardness) have been determined. These relations can be used to predict optimal interlayer dimensions and material properties for any given layer system. Design criteria for this optimization are being laid down.

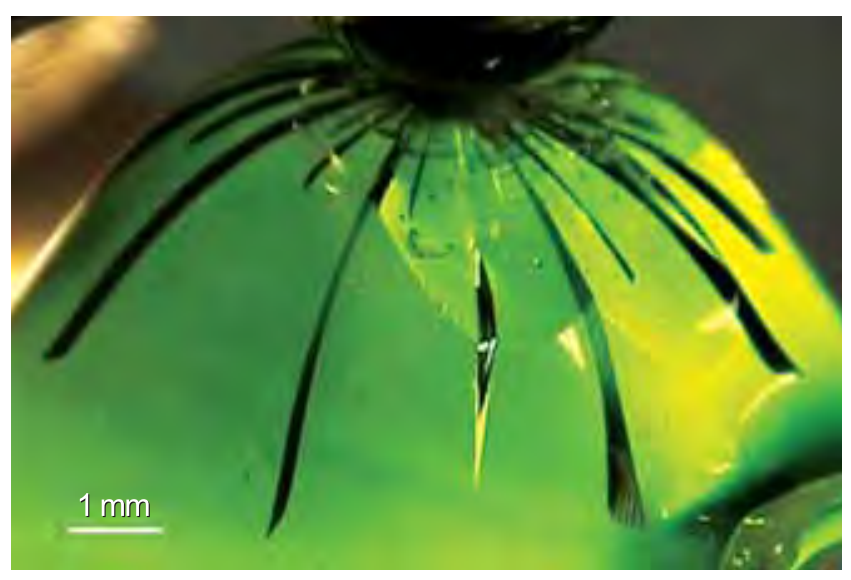

This work has been funded by the National Institute of Dental and Craniofacial Research. Materials scientists, engineers and clinicians are involved. The first five-year stage of this program has been completed. A second five-year stage is under way. Several dental materials companies participate in this program.

\section{Publications}

Brian Lawn's publications can be viewed and downloaded at http://www.msel.nist.gov/lawn/ index.html.

\section{Contributors and Collaborators}

D. Rekow, V. Thompson, Y. Zhang (New York University); I. Lloyd (University of Maryland); A. Pajares, F. Guiberteau, P. Miranda, E. Sanchez (University of Extremadura); M. Bush, X. Hu (University of Western Australia); S. Bhowick, I. Hermann, J.-W. Kim (Guest Scientists); Ivoclar-Vivadent (Norton-Desmarquest); V. Zahnfabrik (Ceramco, Therics) 


\section{Molecular Design and Combinatorial Characterization of Polymeric Dental Materials}

Polymeric dental materials are finding increasing applications in dentistry and allied biomedical fields. As part of a joint research effort supported by the National Institute of Dental and Craniofacial Research and also in collaboration with the American Dental Association Health Foundation Paffenbarger Research Center, NIST is providing the dental industry with a fundamental knowledge base that will aid in the prediction of clinical performance of dental materials.

\section{Joseph M. Antonucci and Sheng Lin-Gibson}

$\mathrm{I}_{\mathrm{o}}^{\mathrm{n}}$ n contrast to current methods that rely on one-specimen-at-a-time measurements, metrologies based on combinatorial and high-throughput (C\&HT) approaches can accelerate fundamental and applied research in dental materials. For dental polymers and their derivatives (sealants, adhesives, restorative composites), many critical properties depend on the chemical, structural, and compositional nature of the initial monomer (resin) system. For multiphase dental materials, e.g., composites, similar factors govern the quality of the interphase between the silanized filler phase and the resin matrix. The objective of this research was to determine the feasibility of adapting C\&HT techniques to measure material properties and screen various experimental resin chemistries for molecular design of novel dental polymers and composites. The technologies developed to enable this research include nanoindentation and the fabrication of single component or multi-variable discrete and continuous gradient films.
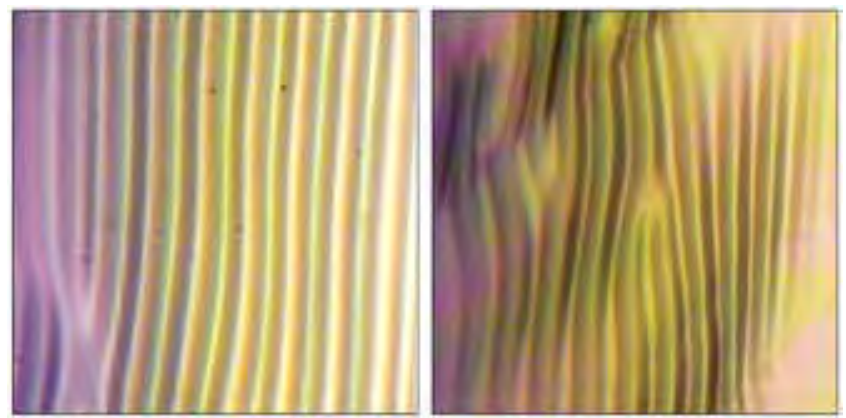

Figure 1: Buckling patterns of BisGMA-TEGDMA in mass ratios of 30:70 (left) and 70:30 (right).

Among the different resin chemistries under investigation, 2D compositional gradients using BisGMATEGDA were selected as the benchmark for developing metrologies and rapid screening techniques for optimizing hardness, shrinkage, and biocompatibility. The elastic modulus was determined by two methods, nanoindentation and SIEBIMM — a strain-induced elastic buckling instability for mechanical measurements test. SIEBIMM on PMMA, a linear polymer, yielded a modulus comparable to that obtained by the 3-point bend test. Buckling patterns from cross-linked BisGMA/TEGDMA films (Figure 1) resulted in moduli with increased variability, i.e., the buckling patterns were not straight, parallel lines. Reasons for this behavior are under study.

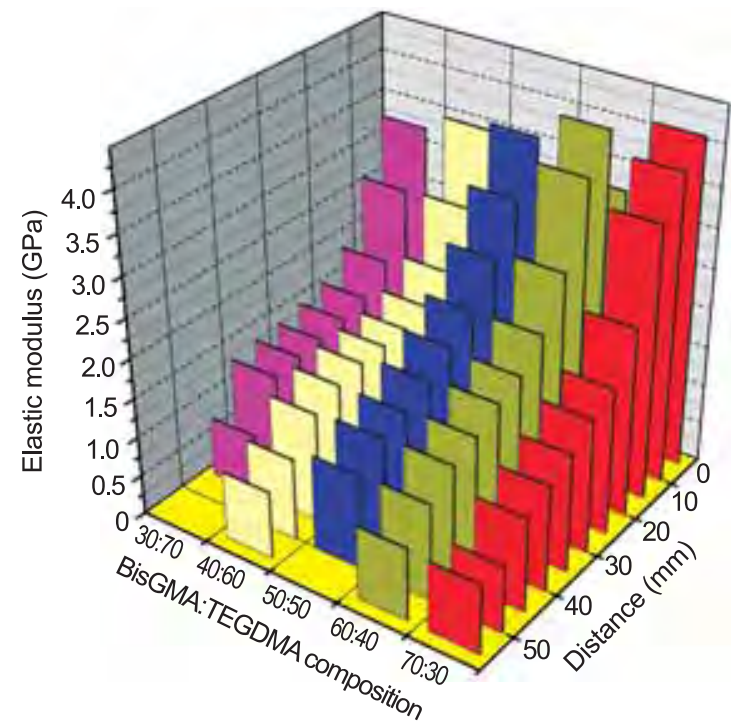

Figure 2: Elastic moduli of photopolymerized BisGMA-TEGDMA of different compositions as a function of irradiation time (represented as distance).

BisGMA-TEGDMA networks with 2D gradients, varying in monomer composition and conversion, were fabricated with a broad conversion range for all monomer compositions. Conversions were measured using near-IR spectroscopy, and elastic modulus and hardness. As shown in Figure 2, the conversion and the mechanical properties correlated well.

Additional techniques with the potential for C\&HT approaches are being evaluated for their ability to screen other properties of dental materials, including the interfacial silane chemistry and cellular response. Studies on the interfacial chemistry have shown that covalent bonding of nanoparticles with the polymerized matrix resulted in well-dispersed composites. To screen the biological response to dental materials, methods to measure cell viability, apoptosis, and gene expression levels as a function of vinyl conversion have been developed.

\section{Contributors and Collaborators}

E.A. Wilder, K.S. Wilson, N.J. Lin, C.M. Stafford, L. Henderson (Polymers Division, NIST); P.L. Votruba-Drzal (Materials and Construction Research Division, NIST) 


\section{Safety and Reliability}

We take for granted that the physical infrastructure around us will perform day in and day out with consistent reliability. Yet, failures occur when these structures degrade to where they no longer sustain their design loads, or when they experience loads outside their original design considerations. In addition, we have become increasingly aware of our vulnerability to intentional attacks. The Safety and Reliability Program within MSEL was created to develop measurement technology to clarify the behavior of materials under extreme and unexpected loadings, to assess integrity and remaining life, and to disseminate guidance and tools to assess and reduce future vulnerabilities.

Project selection is guided by identification and assessment of the particular vulnerabilities within our materials-based infrastructure, and focusing on those issues that would benefit strongly by improved measurements, standards, and materials data. This year, we have worked with the Department of Homeland Security and the Office of Science and Technology Policy in developing the National Critical Infrastructure R\&D Plan, which will provide guidance across much of the national infrastructure. Ultimately, our goal is to moderate the effects of acts of terrorism, natural disasters, or other emergencies, all through improved use of materials.

Our vision is to be the key resource within the Federal Government for materials metrology development as realized through the following objectives:

- Develop advanced measurement methods needed by industry to address reliability problems that arise with the development of new materials;

- Develop and deliver standard measurements and data;

- Identify and address vulnerabilities and needed improvements in U.S. infrastructure; and

- Support other agency needs for materials expertise.

This program responds both to customer requests (primarily other government agencies) and to the Department of Commerce 2005 Strategic Goal of "providing the information and framework to enable the economy to operate efficiently and equitably." For example, engineering design can produce safe and reliable structures only when the property data for the materials are available and accurate. Equally important, manufacturers and their suppliers need to agree on how material properties should be measured.

The Safety and Reliability Program works toward solutions to measurement problems on scales ranging from the macro to the micro. The scope of activities includes the development and innovative use of state-of-the-art measurement systems; leadership in the development of standardized test procedures and traceability protocols; development of an understanding of materials in novel conditions; and development and certification of Standard Reference Materials ${ }^{\circledR}$ (SRMs). Many of the tests involve extreme conditions, such as high rates of loading, high temperatures, or unusual environments (e.g., deep underwater). These extreme conditions often produce physical and mechanical properties that differ significantly from handbook values for their bulk properties under traditional conditions. These objectives will be realized through innovative materials property measurement and modeling.

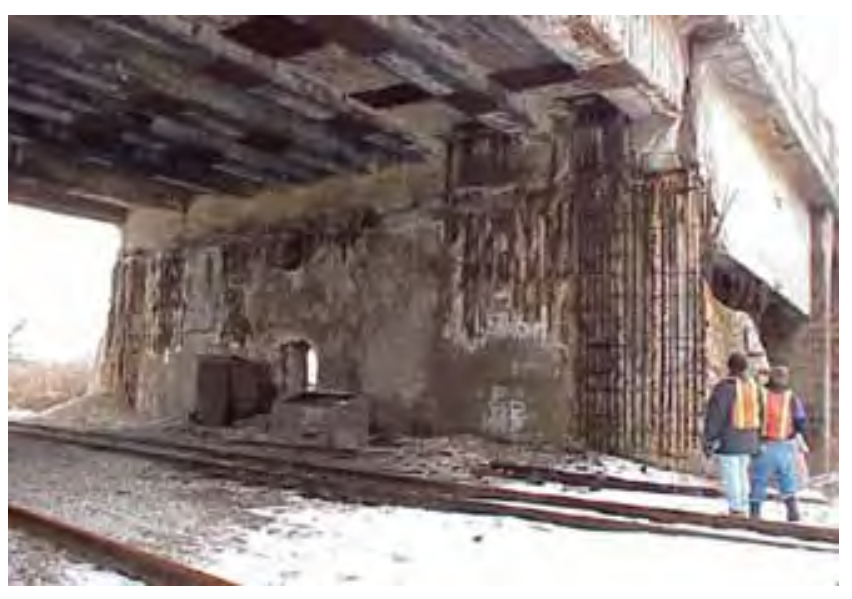

The MSEL Safety and Reliability Program is also contributing to the development of test method standards through committee leadership roles in standards development organizations such as the ASTM International and the International Standards Organization (ISO). In many cases, industry also depends on measurements that can be traced to NIST SRMs.

In addition to the activities above, MSEL provides assistance to various government agencies on homeland security and infrastructural issues. Projects include assessing the performance of structural steels as part of the NIST World Trade Center Investigation, collaborating with both the Department of Transportation and the Department of Energy on pipeline safety and bridge integrity issues, advising the Bureau of Reclamation on metallurgical issues involving pipelines and dams, and advising the Department of the Interior on the structural integrity of the U.S.S. Arizona Memorial.

\footnotetext{
Contact: Thomas A. Siewert (Materials Reliability Division), Frank W. Gayle (Metallurgy Division)
} 


\section{Analysis of Structural Steel from the World Trade Center}

In 2005 NIST completed the three-year Federal Building and Fire Safety Investigation of the World Trade Center Disaster. The investigation addressed many aspects of the catastrophe, from occupant egress to factors affecting how long the WTC towers stood after being hit by the airplanes, with a goal of gaining valuable information for the future. A critical aspect of the investigation was the metallurgical analysis of the recovered structural steel. The analysis included characterization of mechanical properties, failure modes, and structural response determined from photographic evidence.

William E. Luecke, Stephen W. Banovic, Timothy J. Foecke, J. David McColskey, Christopher N. McCowan, Richard J. Fields, Thomas A. Siewert, and Frank W. Gayle

The collapse of the World Trade Center (WTC) 1 towers was the worst building disaster in human history. Engineers, emergency responders, and the nation were largely unprepared for such a catastrophe. The task of the NIST investigation was to determine the details of why and how the towers collapsed. As part of this investigation, the Metallurgy and Materials Reliability Divisions characterized the recovered structural steel.

The project is comprised of five tasks:

1. Collect and catalog physical evidence.

2. Categorize failure mechanisms from visual evidence.

3. Determine steel properties to support modeling.

4. Correlate determined and specified steel properties.

5. Analyze steel to estimate temperature extremes.

Analysis of fracture surfaces of recovered perimeter columns struck by the aircraft showed that the steel

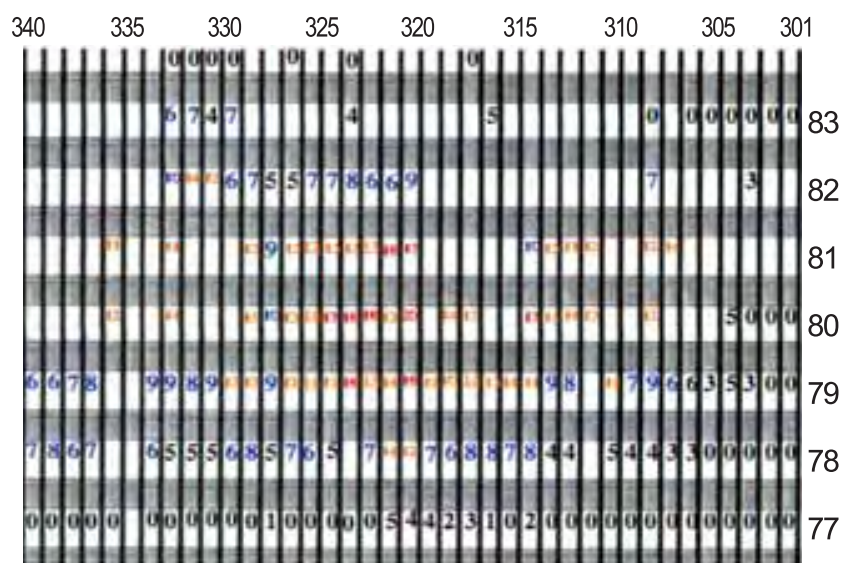

Figure 1: Pull-in map of perimeter columns (in inches) on the east face of WTC 2, 9:03 a.m. remained ductile even at high deformation rates. Data such as these were important in assuring the accuracy of the steel properties supplied to the modeling efforts.

Photographic analysis demonstrated that perimeter columns of WTC 2 began to pull into the building almost 30 minutes before collapse. Our staff developed pull-in maps based on pre-collapse photographs, Figure 1, which were instrumental in constraining the finite element models of the building deformation.

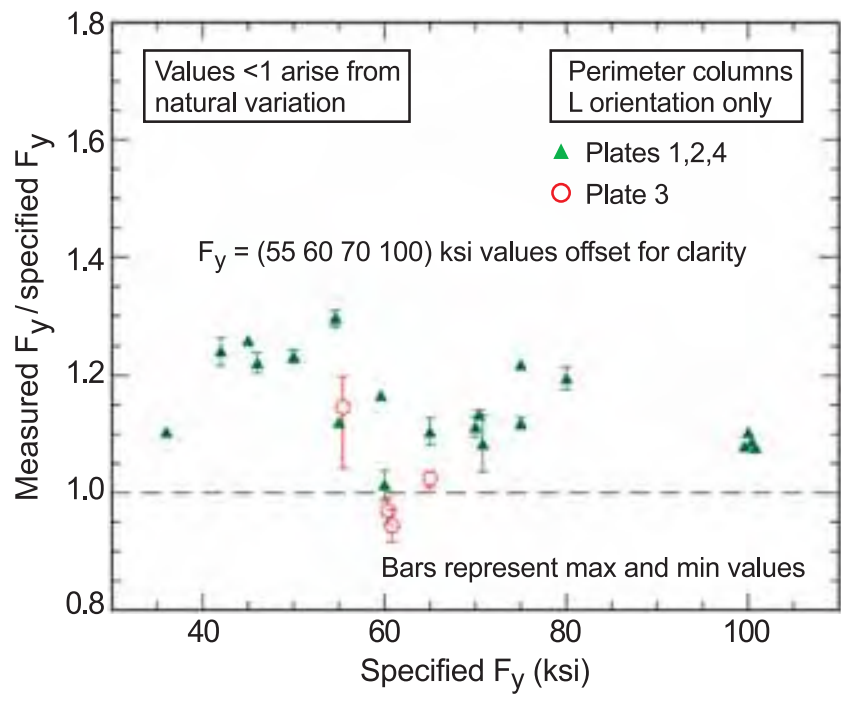

Figure 2: Measured yield strength of perimeter column specimens.

Tensile tests of the dozens of steel types and grades recovered showed that their yield and tensile strengths are consistent with the expected values. In a few cases, the strengths of the NIST-tested specimens were slightly less than called for, but the number of under-strength samples is consistent with the natural variability in steel strength and the damaged state of the recovered steel. Figure 2 shows the ratio of measured to specified yield strength for recovered perimeter column steels.

Photographic evidence indicated that 16 of the recovered perimeter column panels from WTC 1 were exposed to fire before the collapse. Our staff developed a forensic test based on paint cracking due to thermal expansion of the steel. This test placed limits on the time and temperature exposure of the recovered columns. Results indicate that only three locations on these 16 recovered columns reached temperatures above $250{ }^{\circ} \mathrm{C}$.

The final draft of the investigation report was issued for public comment in June 2005.

\section{Contributors and Collaborators}

R. Santoyo (Materials Reliability Division, NIST); M. Iadicola (Metallurgy Division, NIST) 


\section{Infrastructure Reliability: Charpy Impact Machine Verification}

We assist owners of Charpy impact machines in achieving conformance with the requirements of ASTM Standard E 23. We interact with the ASTM Committee responsible for the Charpy impact standard, to improve the service and to maintain a high-quality verification program. We also participate in the activity in ISO Committee TC 164 , so our specimens and procedures remain compatible with the associated international and regional standards.

\section{Thomas A. Siewert}

\section{Technical Description}

$\mathrm{T}_{\mathrm{z}}^{\mathrm{h}}$ he Charpy impact test uses a swinging hammer to assess the resistance of a material to brittle fracture. The absorbed energy is measured from a calibrated scale, encoder, and/or an instrumented striker. The low cost and simple configuration of the test have made it a common requirement in codes for critical structures such as pressure vessels and bridges. This project is handled jointly by the Standard Reference Materials Program (of the Office of Measurement Services), which oversees the administrative aspects of the program, and the Materials Reliability Division, which handles the technical and verification aspects. NIST provides highly characterized Standard Reference Materials ${ }^{\circledR}$ (SRMs) to machine owners and independent calibration services, then evaluates the results of tests of these specimens on their impact machines. Owners of machines that meet the requirements of ASTM Standard E 23 are given a letter of conformance, while owners of nonconforming machines are given recommendations on corrective actions. Our special facilities include three master Charpy impact machines (all $300 \mathrm{~J}$ to $400 \mathrm{~J}$ capacity). These three machines are used to establish certified values for the NIST reference materials sold through the Standard Reference Materials Program Office. In addition, we have several more machines (3 J to $400 \mathrm{~J}$ capacities) that are used for research purposes.

\section{Accomplishments}

Ray Santoyo continues in his role as Charpy Coordinator. We have served about 850 customers in the past year, a slight increase over the year before. The great majority of these machines were within tolerances required by ASTM Standard E 23.

As usual, many customers took advantage of our support services, as shown by over 674 emails, 730 faxes, and 500 phone calls in the first 9 months of FY05. We immediately contact the machine owner if their machine fails to meet the verification criteria. In this contact (by phone, mail, email, or fax), we suggest corrective measures.

NIST's support of ISO Standard 17025 means that we have been reformatting our quality manual for the Charpy program to match the new NIST styles and to fit with the overall NIST Quality Manual (QM-I). Thus, Chris McCowan has refined our Division quality manual (QM-II) and the Charpy program manual (QM-III) to match those of the other Laboratories and Divisions. We expect to perform an independent audit by September 2005 and then formally adopt these new quality manuals.

We helped to organize another international symposium, the Second Symposium on Pendulum Impact Machines: Procedures and Specimens, held in conjunction with the November 2004 meeting of ASTM Committee E 28 in Washington, D.C. We also helped to lead the symposium and contributed to three of the papers. Previous symposia have provided valuable insight into improvements in our program.

This year, we produced the first batch of Izod impact verification specimens, which has been tested and is undergoing statistic evaluation. Soon, it is expected to enter inventory as SRM 2115.

Chris McCowan serves as the Chairman of ISO TC164 SC4 P, on pendulum impact and also as the Chairman of ASTM Subcommittee E28.07 on impact testing.

\section{Contributors and Collaborators}

R. Santoyo (Charpy Program Coordinator); C. McCowan, J. Clark, D. Cyr, C. Dewald, S. Vincent, N. Neumeyer, J. Percell (Materials Reliability Division, NIST); IRMM (Europe); NRLM (Japan); Members of ASTM Subcommittee E 28.07 


\section{Standard Test Methods for Fire-Resistive Steel}

The fires and subsequent collapse of the World Trade Center focused attention on the vulnerability of structural steel to fire. Recently steels designated as "fire-resistive" have become available. This project is developing a standard test method for quantitatively evaluating and comparing the resistance of structural steels to high-temperature deformation.

\section{William E. Luecke and J. David McColskey}

Ill steels lose strength with increasing temperature. $\mathrm{ABy} 600^{\circ} \mathrm{C}$, most structural steels have lost more than half their strength. At intermediate temperatures the strength is independent of time, but above $500^{\circ} \mathrm{C}$, creep, or time-dependent deformation, further reduces the load-carrying capability. To combat this loss of load-carrying capability, structural steel in buildings is insulated to keep it cool in fire.

Fire resistive (FR) steels are intended to be drop-in replacements for existing grades of structural steel. They can meet the same specifications, have similar weldability, cost only marginally more, but have superior elevated temperature strength. Their superior high-temperature properties have the potential to provide extra time for building occupants to escape a fire.

In Japan and Europe, FR steels are qualified based on high-temperature retained yield strength. Although this definition employs a simple, familiar test, because it is a short-term test, it ignores the time dependence of the deformation resistance. Domestic standards for structural components use a critical temperature criterion for failure of steel, so effectively all steels are identical, regardless of high-temperature deformation resistance.

We are studying three possible test methods for standardization. The first is the conventional high-temperature retained yield strength. The second is a slow-rate (several hours to failure) tension test, which should capture the time-dependent deformation effects. The third method is a hybrid of a creep and a conventional tension test in which the test specimen is held under constant load as the test temperature ramps upward linearly. Over a narrow temperature range, which can be approximated as a critical temperature, the deformation rate increases drastically, and the specimen fails. This critical temperature can be used as a measure of the fire-resistance.

One goal of this project is to build on recent worldwide research on similar tests, but to take the proof of concept to a draft standard. Each potential method has advantages, but there has been no research to compare the results of each to the others.
Our research focuses on understanding the limitations, repeatability, and reproducibility of the methods by characterizing several different classes of construction steels. In the near term, after selecting a single method, we will organize an interlaboratory study (ILS) to probe the limitations and establish precision and bias.
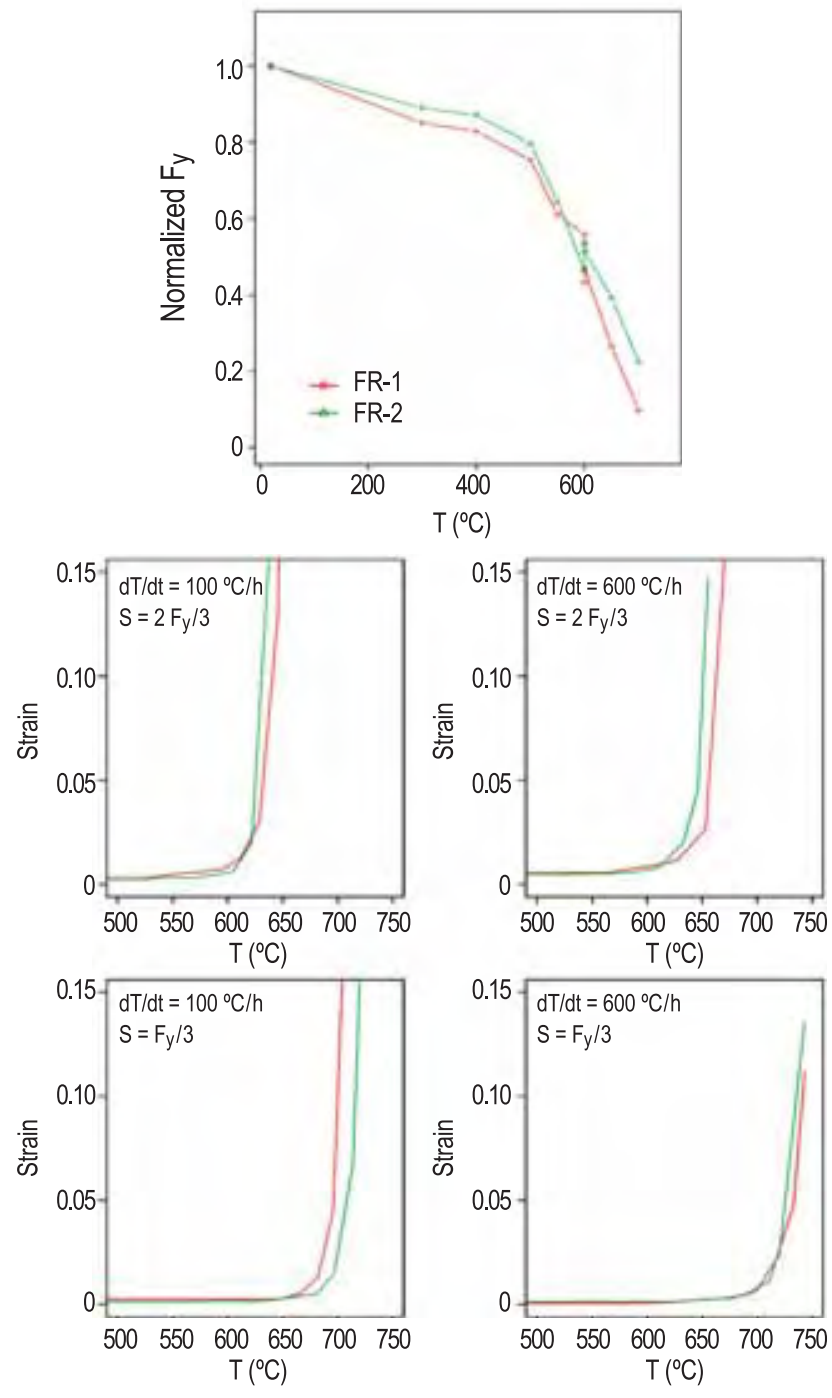

Figure 1: Comparison of tensile and ramp tests.

Figure 1 compares the behavior of two FR steels evaluated using high-temperature, tensile yield strength, $F_{y}$, measured in a high-temperature tensile test and the critical temperature evaluated using the temperature ramp test. Although the normalized yield strengths diverge significantly above $600{ }^{\circ} \mathrm{C}$, the critical temperatures measured in the ramp tests are very similar.

\section{Contributors and Collaborators}

R.J. Fields (Metallurgy Division, NIST) 


\section{Frangible Bullets and Soft Body Armor}

The NIST Office of Law Enforcement Standards (OLES) is currently involved in modifying existing standards for the ballistic resistance of personnel body armor and providing new performance standards for frangible bullets (designed to fully disintegrate on impact with hard surfaces). The deformation behavior of this frangible material when impacting a soft surface such as NIJ Level II or IIIA soft body armor is not as predictable as conventional bullets and requires a better understanding of the high-strain rate deformation behavior of the fabrics used in soft body armor and the various frangible materials.

\section{Stephen D. Ridder and Steven P. Mates}

\begin{abstract}
s part of this multi-year project, the Metallurgy Division is testing Pressed and Sintered $\mathrm{Cu}-\mathrm{Sn}$ (PS Cu-Sn) bullets, one of the frangible ammunition materials selected for testing by OLES. PS Cu-Sn frangible material is made by blending a 9:1 mass ratio of $\mathrm{Cu}$ and $\mathrm{Sn}$ powder with nominal particle sizes of $50 \mu \mathrm{m}$, pressing the powder mixture in molds and then using transient liquid phase sintering to bind the compacts and form a Cu-Sn reaction layer $(\approx 30$ min @ $260{ }^{\circ} \mathrm{C}$ ). Sintering time and temperature are controlled to limit the reaction zone and avoid formation of the less frangible $\alpha$-bronze phase.
\end{abstract}

Microstructural analysis of the as-received PS $\mathrm{Cu}-\mathrm{Sn}$ material has shown that the matrix consists of a mixture of copper particles with varying morphologies - some with equiaxed grain structures and others with $\mathrm{Cu}-\mathrm{P}$ precipitates (possibly the result of copper powder from different suppliers). EDS analysis indicates that the binding region between the copper matrix particles consists of columnar grained $\mathrm{Cu}_{3} \mathrm{Sn}(\varepsilon)$ phase, a more blocky shaped $\mathrm{Cu}_{6} \mathrm{Sn}_{5}\left(\eta^{\prime}\right)$ phase, and unreacted $\mathrm{Sn}$. SEM fractographs of Kolsky Bar specimens have shown brittle fracture in the $\varepsilon$ phase, brittle cleavage in the $\eta^{\prime}$ phase, and ductile failure with micro-void coalescence in the copper matrix adjacent to the intermetallic layers.

The mechanical deformation of PS Cu-Sn frangible bullets has been examined at conventional strain rates and at high strain rates in compression in the NIST Kolsky Bar facility. Kolsky bar tests have been performed to assess the compressive stress-strain behavior at strain rates up to $10^{3} \mathrm{~s}^{-1}$. Dynamic tensile strength has also been measured using the Kolsky Bar to perform diametral compression tests, or Brazil tests. In this test, a disk of the bullet material is exposed to

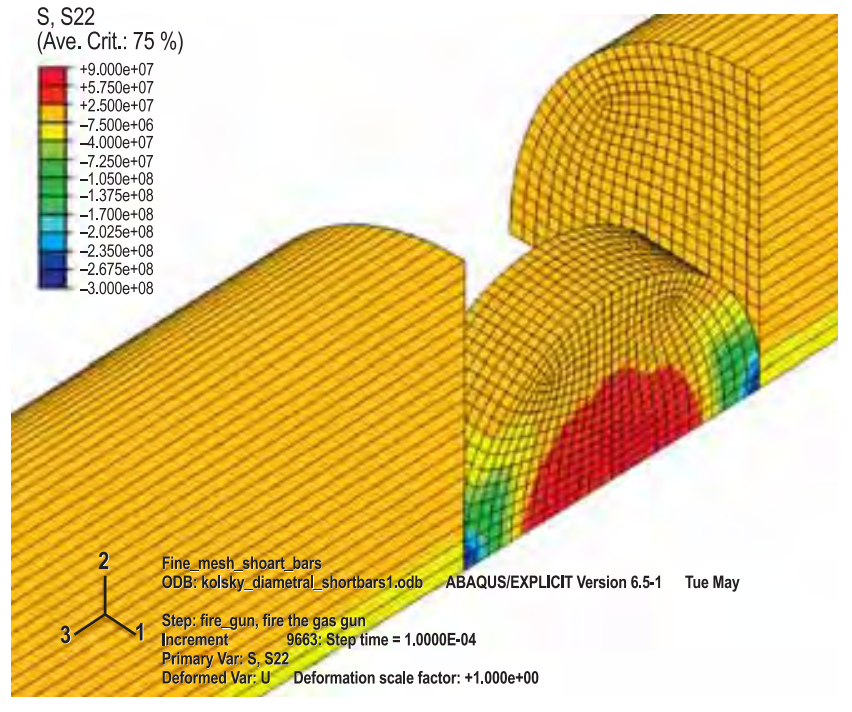

Figure 1: 3-D simulation of diametral test of frangible ammunition cross-section.

a rapidly rising stress pulse along its diameter, which creates a tensile stress perpendicular to the applied load. A crack eventually forms due to this tensile stress, from which the tensile strength can be measured. Typical Brazil tests involve purely brittle materials, and only the elastic deformation behavior of the sample is needed to determine tensile strength. However, in the case of frangible bullet materials, significant plastic deformation was observed before failure. Finite element modeling (Figure 1) was used to determine how plastic yielding affected the evolution of tensile stress in the sample during the test in order to obtain a more accurate estimate of the tensile strength. The high-strain rate plastic flow and tensile strength data can then be used to model the deformation and breakup of a frangible bullet as it impacts a soft armor-like surface.

\section{Expected Impacts}

These data and associated bullet impact modeling efforts will be used by the NIST Office of Law Enforcement Standards in a continuing effort to develop a performance standard for frangible ammunition and in the revision of the National Institute of Justice Ballistic Resistance of Personnel Body Armor Standard.

\section{Contributors and Collaborators}

S. Banovic, R. Fields, L. Levine, L. Ma (Metallurgy Division, NIST); M. Kennedy, R. Rhorer, E. Whitenton (Manufacturing Metrology Division, NIST); T. Burns (Mathematical and Computational Sciences Division, NIST) 


\section{Pipeline Safety: Corrosion, Fracture, and Fatigue}

A critical element of the nation's infrastructure is the more than 3 million $\mathrm{km}$ of natural gas and hazardous liquid pipelines that deliver almost 2/3 of the nation's energy. Following the passage of the Pipeline Safety Improvement Act in 2002, NIST began working with the pipeline industry, DoE, DoI's Minerals and Management Service, and DoT's Office of Pipeline Safety to provide the measurement methods, standards, and data needed to understand corrosion, fracture, and fatigue failure mechanisms in this critical element of the nation's infrastructure.

\section{Richard E. Ricker and J. David McColskey}

fter Congress passed the Pipeline Safety Improvement 1 Act in 2002, NIST, DoE, and DoT developed a memorandum of understanding (MOU) detailing a coordinated program of research, development, demonstration, and standardization. The goals of the program are to continue improvements in the safety and operation of pipelines and related facilities. NIST has a long history of contributions to pipeline safety and, in this project, is responsible for materials research addressing concerns with corrosion, fatigue, and fracture, especially as pipelines are pushed to higher performance using new materials and higher pressures.

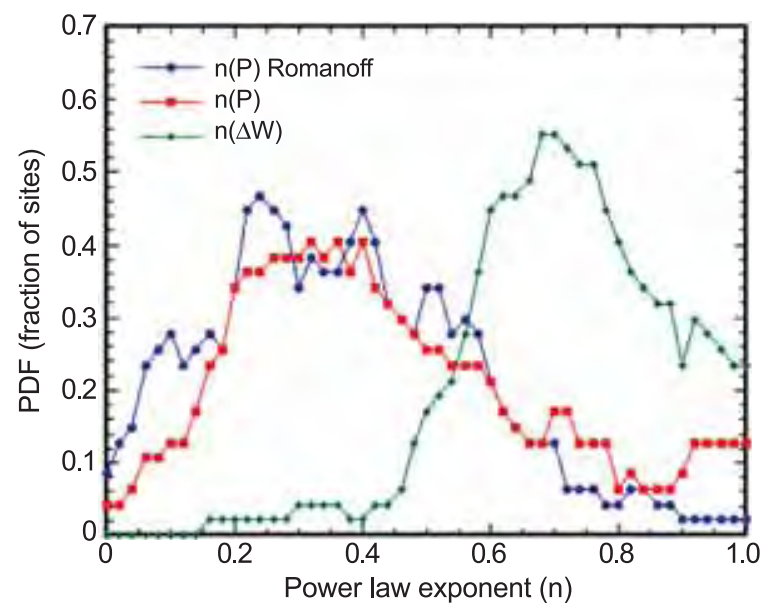

Figure 1: Corrosion analysis (historical data), power law exponents.

At the urging of the Pipeline Research Council International (PRCI) and DoT's Office of Pipeline Safety (OPS), NIST reexamined data from the original NBS underground corrosion studies conducted between 1920 and 1957, involving over 36,000 samples buried at 128 different sites across North America. The original study was found to be thorough and ahead of its time with respect to statistical analysis. Figure 1 compares the results of the power law exponent fit determined in the original study to that determined by modern computer curve fitting routines. This figure shows: (i) strong evidence of non-linear kinetics and decreasing corrosion rates, and (ii) that only minor differences were obtained with modern analysis. In both original and new analyses, the scatter in the data limits the conclusions. The origin of this scatter needs to be understood before less conservative non-linear kinetics can be used for rate models.

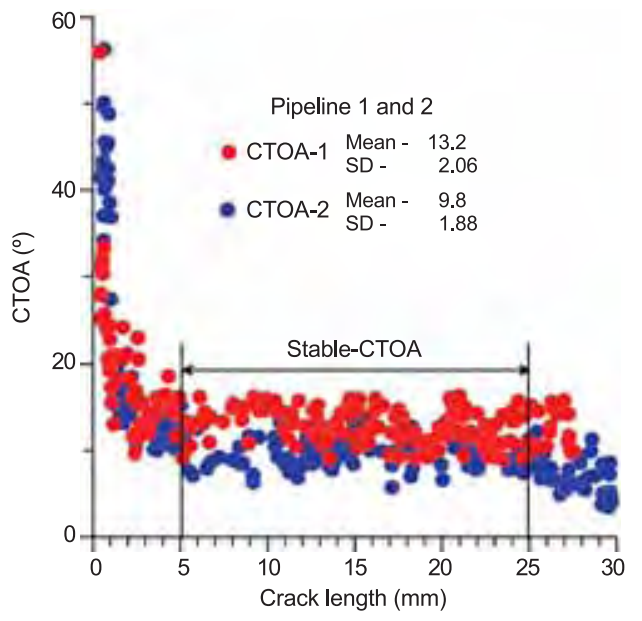

Figure 2: Crack tip opening angle behavior — conventional steel.

Fracture and fatigue studies are underway on both traditional and modern steels. By acquiring and publishing data on these properties, NIST hopes to enable better modeling of performance. The focus of this study is to assess the ability of different pipeline steels to arrest crack propagation. Crack tip opening angle (CTOA) measurements were made on samples cut from the pipeline samples. Figure 2 shows the CTOA measurements on two baseline steels and the region of stable crack growth.

Working with OPS, MMS (DoI), DoE, PRCI, American Gas Association, the Gas Technology Institute, ASTM Intl. NACE Intl. CANMET, and the National Energy Board (Canada), NIST organized a workshop on Advanced Coatings for Pipelines and Related Facilities held at NIST June 9-10. Sixty representatives of the pipeline industry, suppliers, and government agencies from the U.S., Canada, and the UK attended this meeting. NIST also assisted OPS in organizing the Government/Industry Pipeline R\&D Forum in Houston, Texas, March 22-24, 2005.

\section{Contributors and Collaborators}

F. Gayle, T. Foecke, S. Mates, R.J. Fields, C. Handwerker (Metallurgy Division, NIST); T. Siewert, C. McCowan (Materials Reliability Division, NIST); M. Smith (PRCI); C. Sames (AGA); J. Merritt, S. Gerard, R. Smith (DoT/OPS); C. Freitas, R. Anderson, D. Driscoll (DoE); M. Else (DoI/MMS) 


\section{Polymer Reliability and Threat Mitigation}

This project is developing metrologies and predictive models to test and predict the long-term reliability of polymers used in ballistic resistant armor and machine readable travel documents. Use of these methods and models will enable one to monitor the performance of polymeric materials while in use, elucidate how environmental and mechanical factors influence performance, and provide a basis for estimating durability and establishing care procedures.

\section{Chad R. Snyder, Gale A. Holmes, and Walter G. McDonough}

\section{Ballistic Resistant Armor}

Tn response to an apparent failure of ballistic resistant 1 armor during first responder use, NIST's Office of Law Enforcement Standards initiated a research program designed to strengthen the certification process of these protective devices. We are working to identify and develop analytical metrologies for quantifying the mechanical properties and degradation pathways of ballistic fibers that comprise this armor, with the ultimate goal being an estimate of vest durability and care procedures.
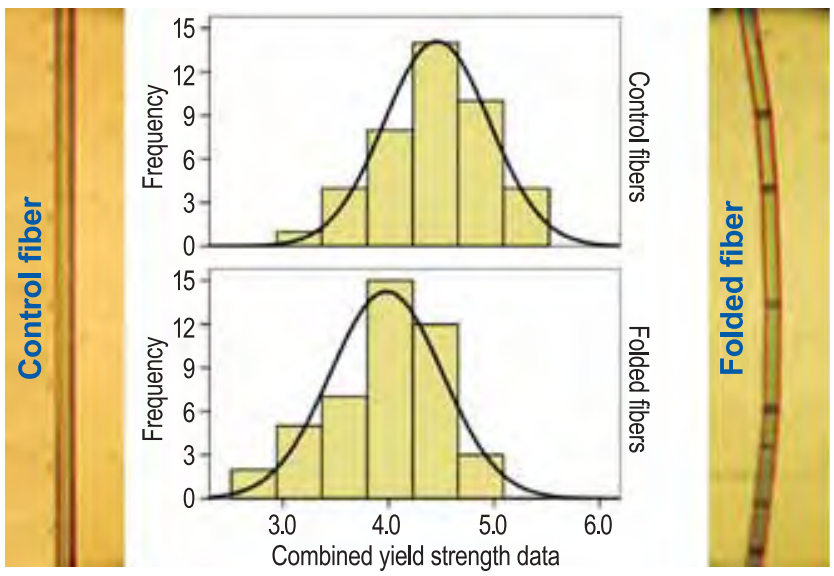

Figure 1: Top and Left: Micrograph and yield strength data obtained from modified single fiber test on an unfolded $P B O$ fiber; Bottom and Right: Micrograph and figure for a folded PBO fiber.

This year, we have made considerable progress on multiple fronts towards these goals for poly(benzoxazole) (PBO) ballistic fibers. The modified single fiber fragmentation test, developed last year, was used to examine the effect of fiber fatigue on ballistic resistance. Figure 1 shows the effect of folding on the morphology and mechanical properties of a PBO fiber. Our analysis suggests that, for this case, folding resulted in an estimated $>10 \%$ reduction in overall ballistic performance, as quantified through the method of Cuniff and Auerbach. Ongoing research is examining the effects of fold radius as well as the effects of repeated folding. ${ }^{[1]}$

Complementing our mechanical properties studies, our research into the degradation pathways of PBO and PBO-like materials has made significant headway. In addition to completion of our review article, ${ }^{[2]}$ we have synthesized the model compounds 2-phenylbenzoxazole and bis-1,4-(2-benzoxazolyl) phenylene, and their hydroxy analogs, and we are currently analyzing, through matrix assisted laser desorption/ionization (MALDI) mass spectrometry, the degradation products resulting from exposure to our newly acquired solar simulator.

\section{Machine Readable Travel Documents}

As indicated in an October 14, 2004 press release from the U.S. Government Printing Office (GPO), NIST is testing the candidates for the new U.S. electronic passports for their ability to meet durability, security, and electronic requirements. This new technology will eventually be incorporated into electronic U.S. passports to enhance the security of millions of Americans traveling around the world. At the request of the U.S. Department of State, we participated in the WG3 (Working Group 3 of ISO) meeting of the Document Durability Task Force for the EPassport in Tsukuba, Japan. The purpose of the task force meeting was to update the participants on the status of the Test Specification for Machine Readable Travel Documents (MRTD). The main recommendation from the meeting was that any proposed test specification by ISO would serve as the guideline to help nations writing requests for proposals to develop their own MRTDs. Also, the task force chairs have decided to use more complex testing sequences to better represent real-world applications; this was in line with the recommendations made by NIST.

\section{References}

1. P.M. Cunnif and M.A. Averback, 23rd Army Science Conference, Assistant Secretary of the Army (Acquisition, Logistics and Technology), Orlando, FL (Dec. 2002).

2. G.A. Holmes, K. Rice, and C.R. Snyder, Journal of Materials Science, in press.

\section{Contributors and Collaborators}

J. Dunkers, C.M. Guttman, K. Flynn, J. Kim, F.A. Landis, D. Liu, W. Wallace (Polymers Division, NIST); D. Novotny, J. Guerrieri, G. Koepke, M. Francis, N. Canales, P. Wilson (Electromagnetics Division, EEEL); K. Rice (Office of Law Enforcement Standards, EEEL); T. Dang (Air Force Office of Scientific Research) 


\section{Facilities and Capabilities}

\section{Advanced Measurement Capabilities} UHV STM/AFM

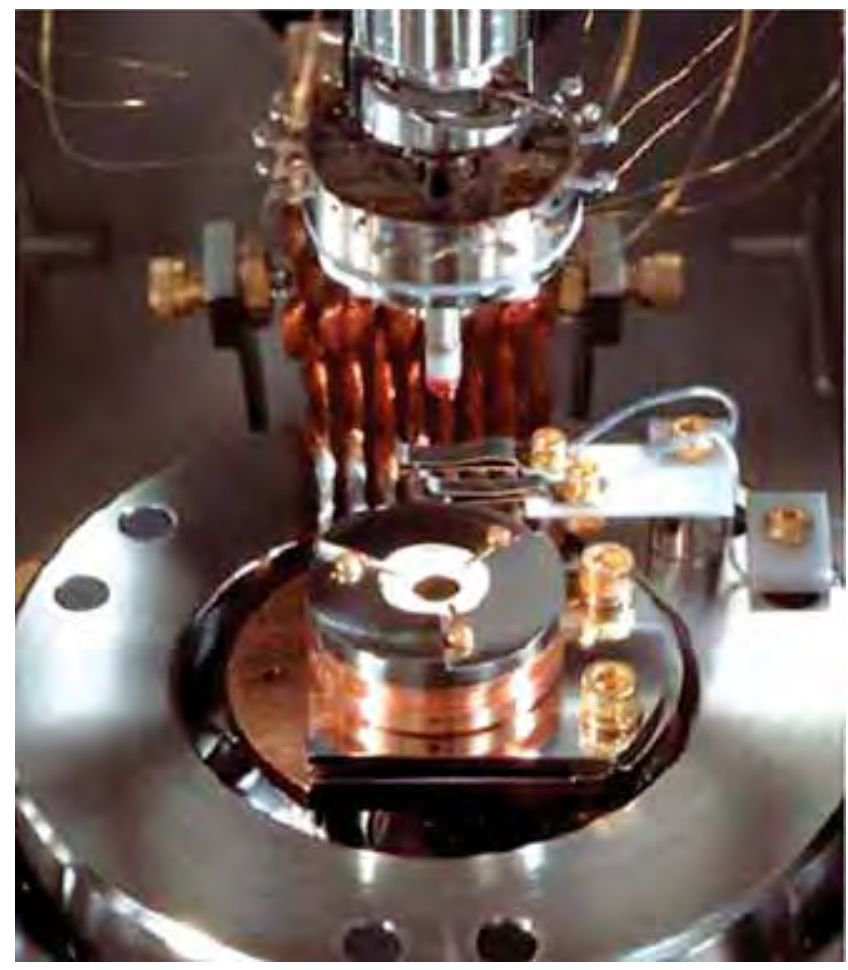

Figure 1: Ultrahigh vacuum STM/AFM apparatus.

Enhanced by the superior vibration isolation and clean room environment in NIST's new Advanced Measurement Laboratory (AML), our UHV STM/AFM system, Figure 1, provides atomic imaging and force measurement with an unprecedented resolution and accuracy. Combined with our conventional AFM, equipped with a "triboscope" attachment, we are able to image and manipulate surface features and measure a wide range of material characteristics and properties important in nanodevice operation. As a result, we now are leading fundamental metrological efforts, working with device and magnetic hard disk industries, as well as academic institutions, to establish reliable measurement methods, calibration artifacts, and infrastructural support for current and future industries.

\section{High Resolution X-ray Metrology}

Powder and single crystal $\mathrm{x}$-ray diffraction are widely used in industry, research facilities, and academia as one of the principal means of characterizing materials. Both techniques yield a wealth of information on the crystallographic and microstructural character of the specimen. The powder diffraction method has the virtue that it can probe a continuous sequence of crystallographic reflections with a single scan in angular space, while single crystal methods can be adapted to high-resolution analysis of materials such as thin films.

However, results from both powder and high-resolution techniques are affected by a complex optical aberration function that is specific to the diffraction optics and goniometer assembly used in the experiment. NIST Standard Reference Materials (SRMs) are the recognized means by which these aberrations may be characterized to achieve improved measurement accuracy. To address these issues, Dr. James Cline of the Data and Standards Technology Group conceived and designed the Ceramics Division Parallel Beam Diffractometer (CDPBD).

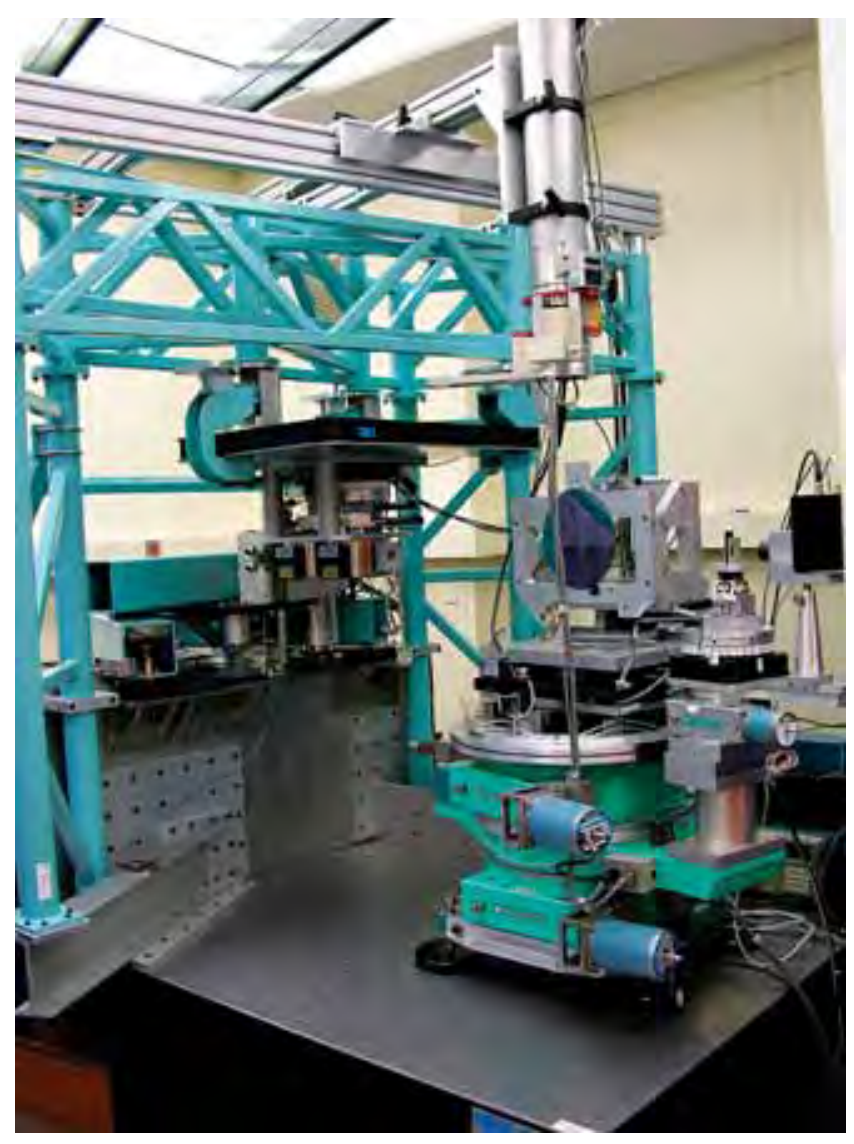

Figure 2: The Ceramics Division Parallel Beam Diffractometer in the AML.

The CDPBD, Figure 2, was designed and built specifically to perform traceable measurements on powder and thin film specimens. Installed in the new NIST Advanced Measurement Laboratory, this facility provides the environmental and temperature controls requisite for a new generation of NIST SRMs that will enable unprecedented measurement accuracy in a highly competitive, data conscious, materials research community. 


\section{X-ray Absorption Spectroscopy}

Advances in our x-ray absorption spectroscopy facilities have achieved an unrivaled capability enabling our Characterization Methods Group (CMG) to address a remarkably broad range of challenging structure and chemistry issues at the forefront of materials science research today. Through the application of a truly unique combination of beamline facilities, we are able to examine characteristics of surfaces, interfaces, and bulk materials in a manner heretofore inaccessible.

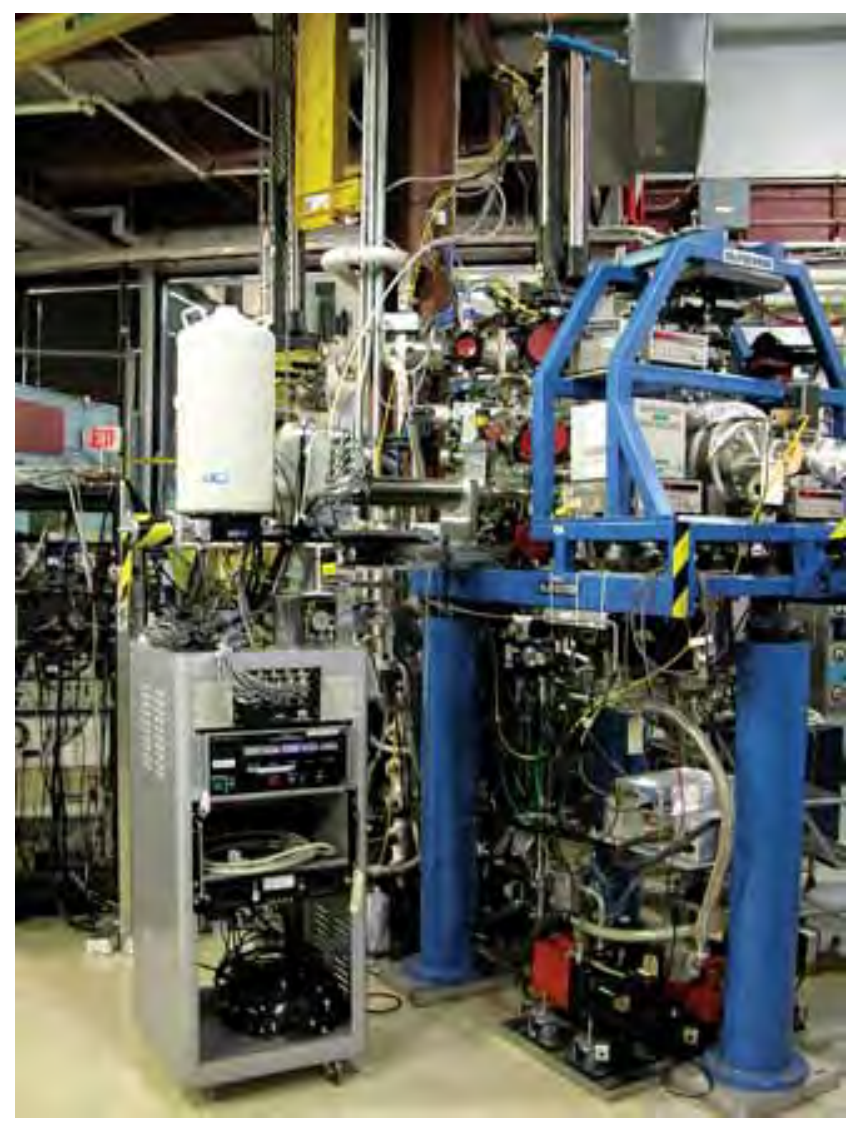

Figure 3: The soft x-ray spectroscopy station on beamline U7A.

To achieve this capability, CMG, led by Dr. Daniel Fischer, brought together a suite of three unique high-throughput $\mathrm{x}$-ray spectroscopy beamlines (designated U7A, X24A, and X23A2). Housed in the National Synchrotron Light Source located at Brookhaven National Laboratory in New York, these beamlines, taken together, can easily examine nearly all of the naturally occurring elements in the entire periodic table. This year, the capabilities of beamline U7A, used for soft x-ray materials science applications, were significantly enhanced by the addition of a 14 element, state-of-the-art, $\mathrm{Si}(\mathrm{Li})$ fluorescence yield detector, providing best-in-the-world resolution. Further enhancement of U7A, Figure 3, was achieved through the installation of a 6-axis manipulator that enables the exciting prospect of molecular alignment studies.
Building on these capabilities, CMG has initiated a long-term plan, co-funded with Sandia National Laboratory, for establishing a variable energy XPS (x-ray photoelectron spectroscopy) and NEXAFS/EXAFS (near edge/extended $\mathrm{x}$-ray absorption fine structure) scientific program utilizing beamline X24A. A new, fully automated materials science end-station is planned, modeled after the very successful high throughput attained on U7A. The emphasis for this work will be on the use of variable energy XPS for chemical depth profiling, sub-surface chemistry, and interface chemistry.

\section{Combinatorial Thin Film Laboratory}

A new laboratory for the synthesis of inorganic combinatorial thin-film libraries is under construction. Combinatorial methodologies can greatly accelerate the optimization of materials in complex systems and, thus, their introduction into commercial products. The Ceramics Division's state-of-the-art combinatorial deposition tool will have dual capability, featuring sputtering as well as pulsed laser deposition (PLD) chambers. The tool will be used to synthesize libraries of multilayer structures, such as advanced complementary-metal-oxide-silicon (CMOS) gate stack structures, in situ, for metrology development and characterization studies.

\section{Tissue Engineering (TE) Bioreactors}

The Center for Mechanical Behavior of Biological Materials (CMBBM) has developed unique bioreactors that allow for experiments to optimize the variables that

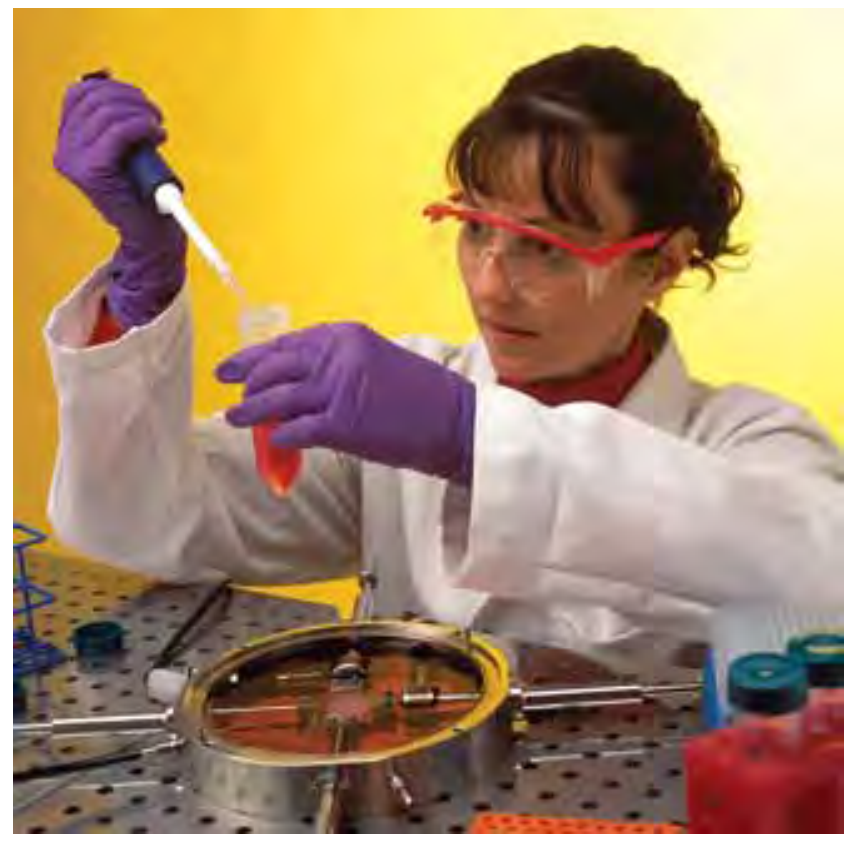

Figure 4: Tammy Oreskovic adds cells to a tissue-engineered scaffold. 
control the mechanical properties of the engineered tissue. The bioreactors that have been developed provide mechanical stimulation to a TE construct and measure mechanical properties while the tissue is being grown in the bioreactor. Cyclic, mechanical stimulation of TE constructs is necessary to make the constructs more like natural tissue. In these bioreactors, we can stimulate a planar sheet of tissue/scaffold construct with an arbitrary waveform. The reactor can be configured to apply a given force or a given displacement. The bioreactor is equipped with actuators, load cells, and view ports to conduct biaxial stress-strain tests without removing the sample from the reactor. We are equipped with the necessary equipment to culture tissue and assess its growth and viability.

\section{Optical Trap}

We have assembled an optical trap that uses a scanned laser at $1064 \mathrm{~nm}$ as the trapping source. The long wavelength allows us to make measurements on biological samples with minimal damage to cellular proteins. The scanned laser system provides multiple time-shared traps and movement of trapped objects so that transient mechanical measurements can be made. The laser operates from $100 \mathrm{~mW}$ to $500 \mathrm{~mW}$, providing a range of trap stiffnesses. Trap stiffnesses on the order of $10^{-6} \mathrm{~N} / \mathrm{m}$ are typical, which allows mechanical measurement of cells, subcellular fibers and structures, DNA strands, and assembled nanoparticles.

\section{Crack-Tip Opening Angle Mechanical Testing}

Better measurements of the tendency to stop a running crack in a structure come from a new test called crack-tip opening angle (CTOA). This test measures the angle at which a crack progresses at a stable rate through a material with a fatigue precrack. It has substantial advantages over its predecessor, the crack-tip opening displacement test, by allowing multiple measurements directly at the crack tip. We are using our system to measure the crack arrest tendencies of a variety of pipeline steels. Improvements that we make in the procedures are being fed back to the ASTM Committee (E08.08) responsible for its development and promulgation.

\section{MEMS Fabrication Facility}

The fabrication facility at NIST-Boulder is a 200 square meter multifunction class 100 clean room capable of $50 \mathrm{~nm}$ linewidths. It is currently used to fabricate micro- and nanoelectromechanical systems (MEMS/NEMS/BioMEMS) which are essential to much of the ultra-sensitive equipment designed and used across many divisions at NIST.

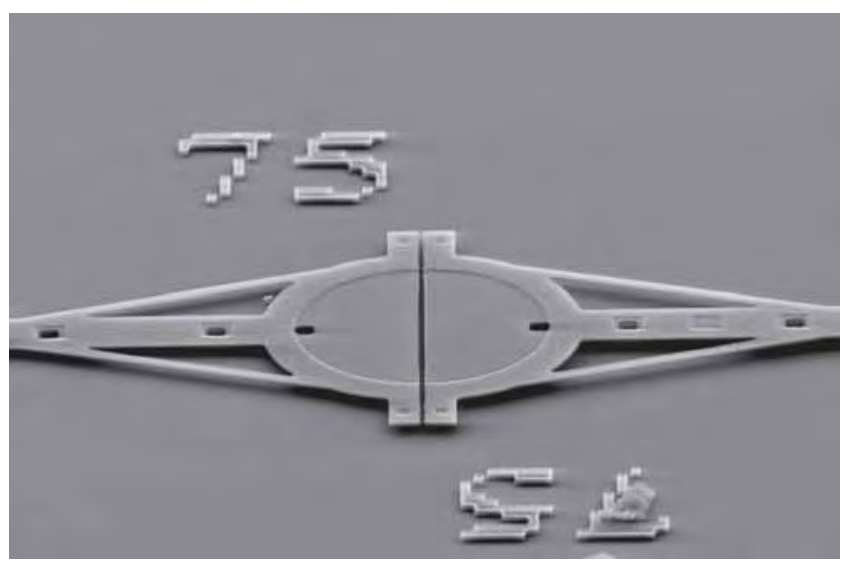

Figure 5: BioMEMS devices fabricated at NIST allow the measurement of single-cell mechanical properties.

From start to finish, all aspects of small-scale fabrication are available within the NIST facility. The facility includes: computer-aided design (CAD), a pattern generator (PG), a $5 \mathrm{x}$ wafer stepper or a $1 \mathrm{x}$ contact printer with backside alignment capability, an electron beam lithography system, various sputter and e-beam deposition tools, as well as an electron cyclotron resonance (ECR) system for low-temperature plasma-enhanced chemical vapor deposition (PECVD), a multiuse chemical vapor deposition (CVD) furnace bank, various reactive ion etching (RIE) tools, a $\mathrm{XeF}_{2}$ isotropic etcher, a chemical mechanical polisher (CMP) for planarization, and a deep reactive ion etcher (DRIE). The facility is professionally staffed by experienced and knowledgeable NIST researchers.

\section{Biaxial Membrane Test Capabilities}

The Materials Reliability Division in Boulder, Colorado, has a program in measuring the mechanical behavior of biological materials. One facet of that program is to measure the biaxial, load-displacement behavior of membrane-like tissue. That is accomplished with a bubble test fixture and a computer-driven system to pressurize the specimen and collect images from four cameras. The multiple camera angles (3 side views + stage rotatability, and 1 top view) allow capture of anisotropic displacement behavior. We have two different sized fixtures, 2.38 and $20.0 \mathrm{~mm}$ in diameter, so we can test materials of different thicknesses while maintaining the 1:10 thickness-to-diameter ratio required for a valid bubble test. All tests on natural tissue are conducted in a $37^{\circ} \mathrm{C}$, phosphate-buffered saline water bath to sustain cell viability.

\section{Laboratory Setup and Establishment of Cell Lines}

The biological laboratory in the Materials Reliability Division has approximately 400 square feet available 
within two adjacent lab areas. A fully equipped tissue culture facility is available for culturing vascular smooth muscle cells and future osteoblast-like and fibroblast cell lines. Also, a flow bioreactor for mechanical evaluation of biological scaffold materials with a specially modified incubator is available. Laboratory equipment includes a biological laminar flow hood with ultraviolet lights, two water-jacketed $\mathrm{CO}_{2}$ incubators, benchtop centrifuge, liquid nitrogen storage vessel, inverted microscope with fluorescent capabilities and integrated firewire camera and analysis software, refrigerator $\left(4^{\circ} \mathrm{C}\right) /$ freezers $\left(-20{ }^{\circ} \mathrm{C} \&-50{ }^{\circ} \mathrm{C}\right)$, and an automated plate reader. Additionally, a probe station for conducting Bio-MEMS testing and single-cell experiments occupies one of the lab areas.

\section{Ultrasonic Imaging and Measurement Facilities}

Our ultrasound measurement equipment includes:

- Range of ultrasonic transducers ( $<1 \mathrm{MHz}$ up to $150 \mathrm{MHz}$;

- Calibrated hydrophone (up to $20 \mathrm{MHz}$ );

- $150 \mathrm{MHz}$ square-wave pulser/receiver;

- 200 MHz negative-spike pulser/receiver;

- Arbitrary waveform generator (up to $15 \mathrm{MHz}$ );

- Broadband amplifier (>50 dB up to $400 \mathrm{MHz}$ );

- High-frequency digitizer (1 GHz - 8-bit);

- Three-axis automated motion control system;

- Automated acquisition system;

- Temperature-controlled immersion baths;

- Custom specimen fixtures.

The acoustic microscope has been used to measure properties of bone, cartilage, arterial tissue, and imaging-enhancing bubbles, to date.

\section{Microfluidic and Particle Processing Facility}

The Polymers Division's new microfluidic facility enables entirely new research programs in polymer chemistry, nanoparticle assembly, and complex fluids metrology. This capability stems from new fabrication methods accommodating organic complex fluids, multilayer architecture integrating functions, and flow simulations central to device design. A suite of in-situ chemical and physical measurements are brought to bear, including Raman spectroscopy, fluorescence, high-speed image analysis, electronic sensing, and rheometry. On-chip processing and production methods comprise controlled radical synthesis, droplet formation and handling, and particle manipulation and assembly.

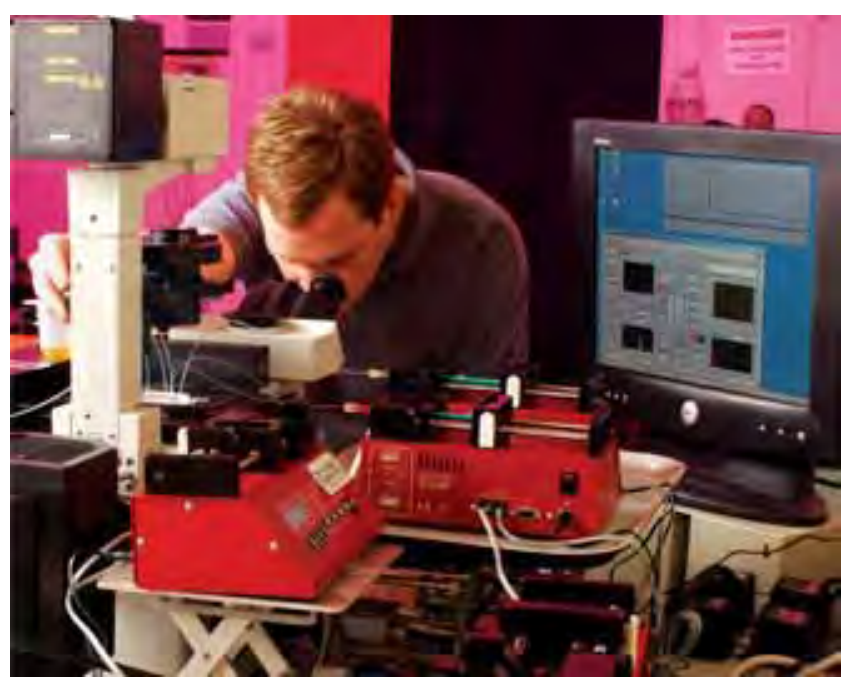

Figure 6: Phillip Stone using microfluidic interfacial tensiometer.

Two technological problems currently under examination by exploiting these high-throughput metrologies are polymerization-induced shrinkage of dental resins and interfacial tension between two liquids. These applications rely on our capability to measure two-component flow, droplet chemistry, and droplet-shape dynamics. In addition, high-frequency on-chip particle size analysis, using embedded electrodes, is a feature of a more complex device that integrates particle counting and analysis, encapsulation, and automatic valve triggering. These capabilities will enable construction of complex nanomanufacturing operations.

\section{Small Angle X-ray Scattering Facility}

As pattern sizes in the semiconductor electronics industry decrease to sub-50 nm dimensions, techniques

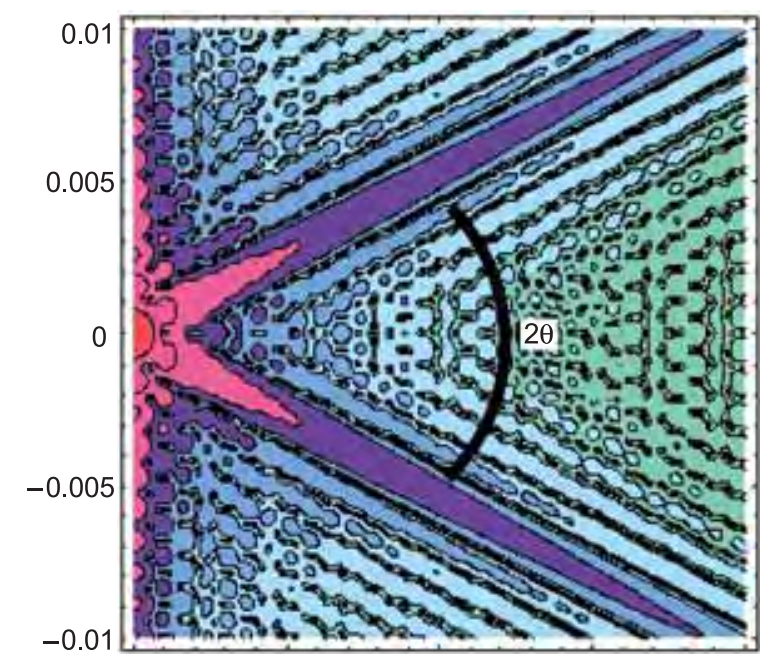

Figure 7: Modeled CD-SAXS data. 

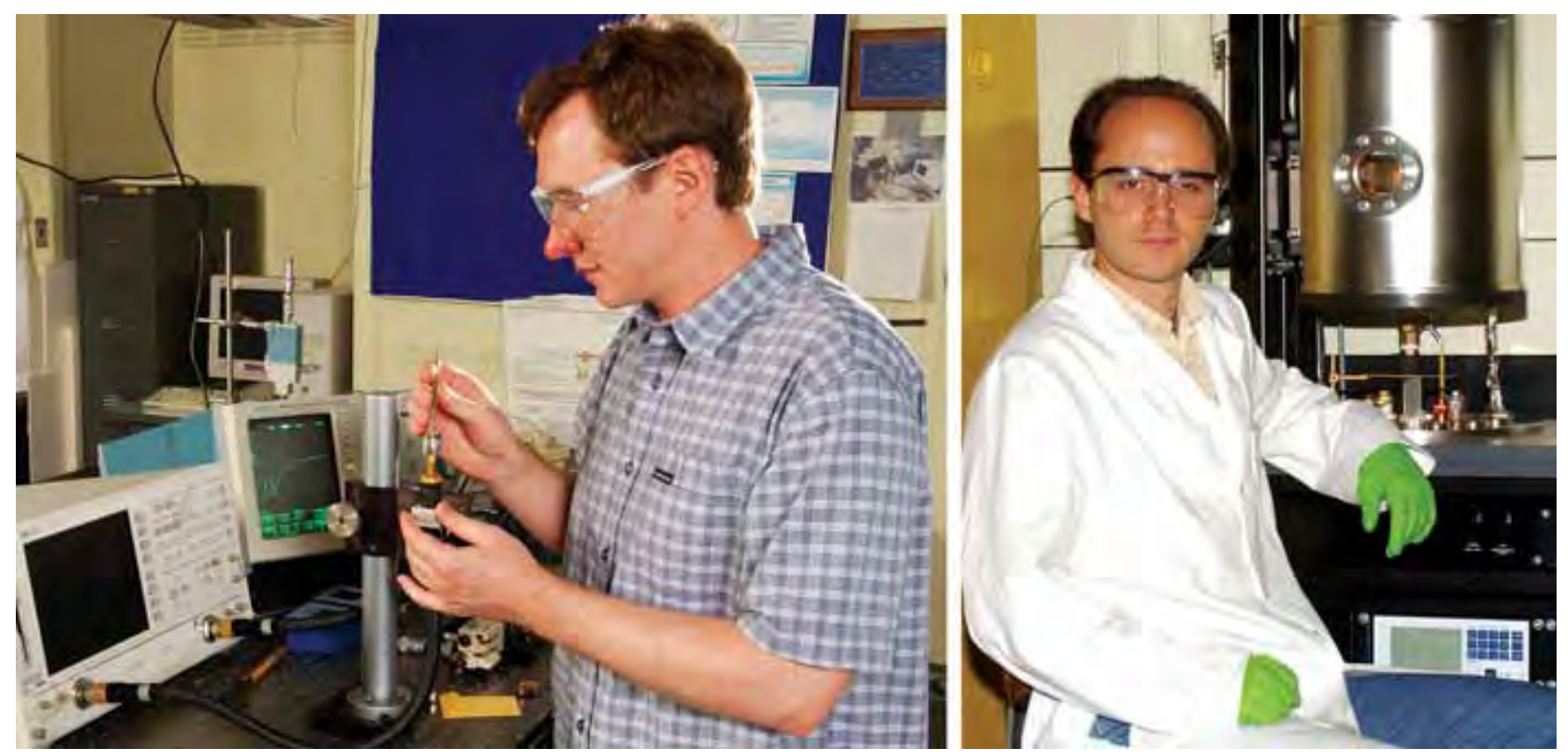

Figure 8: Oleksiy Anopchenko (left) and Dean DeLongchamp (right) at Organic Electronic characterization and preparation facilities.

such as critical dimension-scanning electron microscopy (CD-SEM) face significant technical hurdles in quantifying parameters such as Line Edge Roughness (LER). NIST is evaluating the potential application of small-angle x-ray scattering (SAXS) as a metrology tool for both process development and the production of nanoscale dimension standards. The Polymers Division is building a laboratory scale SAXS system to measure with sub-nanometer resolution, quantitatively and non-destructively, critical dimensions and feature shape of patterns on production scale test samples. This system will use a highly focused molybdenum source with a wavelength where silicon is transmissive and a two-dimensional detector for the evaluation of critical dimension, sidewall angle, and statistical deviations across large areas for dense high-aspect ratio patterns with sub-50 nm dimensions. In addition to these capabilities, this facility will include state-of-the-art SAXS equipment including a copper source and sample handling equipment for the measurement of nanostructured materials, fibers, and polymer solutions.

\section{Organic Electronics Competence}

In 2005, the Polymers Division led a successful 5-year competence proposal, "Metrology to Enable the Realization of Organic Electronics Devices,” in collaboration with the Ceramics Division, the Electronics and Electrical Engineering Laboratory (EEEL), and the Chemical Science and Technology Laboratory (CSTL). In this project, NIST plans to develop an integrated and interdisciplinary suite of metrologies correlating device performance with the structure, properties, and chemistry of critical materials and interfaces. NIST will guide the development of standard test methods and provide the fundamental measurements needed for the development of materials and processes to realize the potential of organic electronics. Several new capabilities have been added at NIST to achieve these goals, including the design of a controlled atmosphere glove box train, a programmable, shielded probe station, and new optical spectroscopy equipment. These facilities, plus the clean room facility available in the Advanced Measurement Laboratory, allow NIST to measure both the structure of materials and device performance.

\section{MSEL Electron Microscopy Facility}

The MSEL Electron Microscopy Facility consists of two transmission electron microscopes, three scanning electron microscopes, a specimen preparation laboratory, and an image analysis/computational laboratory.
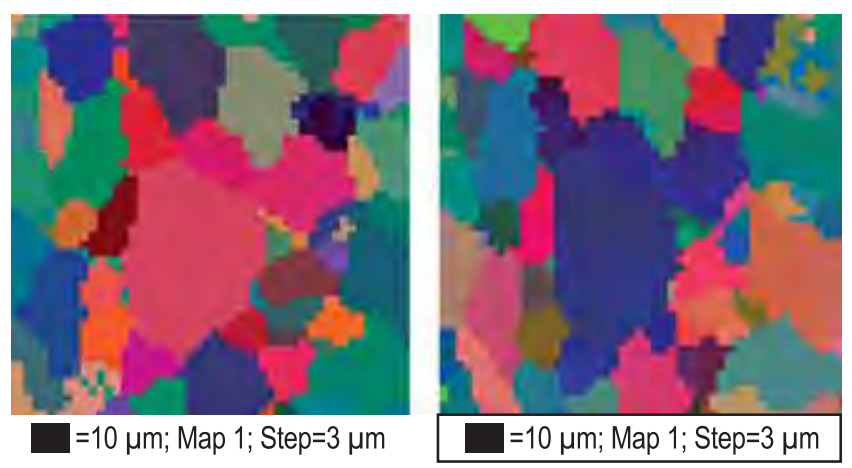

Figure 9: Evolution of texture (EBSD maps) in DQSK steel sheets. Initial conditions (left) and after $10 \%$ uniaxial tensile strain (right). 
The JEM3010 TEM resolves the atomic structure and employs an energy selecting imaging filter (IF) and $\mathrm{X}$-ray detector (EDS) for analytical characterization of thin foil specimens. The S-4700-II FE-SEM employs electron backscattered diffraction/phase identification (EBSD) and EDS systems to characterize the texture and composition of materials.

Highlights from the EM Facility for FY2005 include:

- A new stage compatible with EBSD acquisition will allow analysis of texture evolution under dynamic loading (tensile/compressive) conditions (see Figure 9).

- New forward scatter detectors for the FESEM-EBSD camera allow simultaneous, multiple mode acquisition of secondary and backscattered electrons.

\section{Thin Film Deposition Facility}

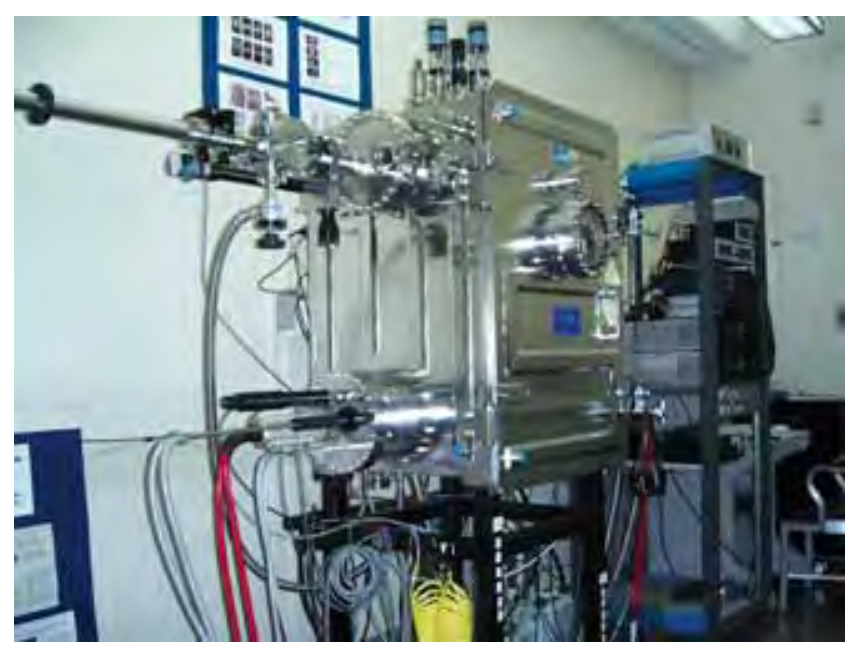

Figure 10: The combinatorial thin-film deposition system has a base vacuum in the low $10^{-8}$ Torr $\left(10^{-6} \mathrm{~Pa}\right)$ range and is equipped with a load-lock for specimen transfer.

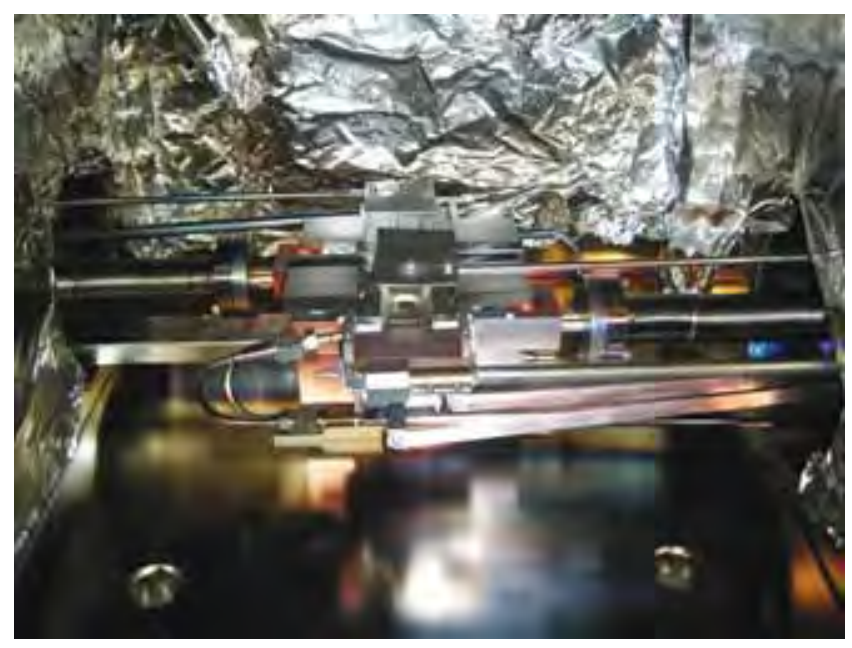

Figure 11: The two sources in the combinatorial thin-film deposition system are each capable of depositing up to six different materials in a single deposition run.
The Thin Film and Nanostructure Processing group of the Metallurgy Division has two high-vacuum, electron-beam evaporator systems for fabrication of thin-film and multilayer thin-film specimens. One system has three independent 15 cc sources and is used for deposition of thin films and multilayer thin films of materials from lower melting metals like aluminum, silver, and copper up to refractory metals like ruthenium, iridium, and tungsten.

The other system (Figure 10) has two independent sources (Figure 11), each capable of depositing as many as six different materials in a single deposition run. With its two-axis, computer-controlled specimen shutter, it is used for fabrication of combinatorial-layered as well as compositionally graded specimens.

\section{Sheet Metal Formability Station}

The Metallurgy Division Materials Performance Group is using the Sheet Metal Formability Station to develop two new formability tests. The closed-loop servo-hydraulic system has $500 \mathrm{kN}$ capacity for both the ram and blank hold-down, and it is capable of deformation rates up to $30 \mathrm{~s}^{-1}$. A unique and important capability is that the system is outfitted with an in situ $\mathrm{X}$-ray diffraction system that makes it possible to measure the stress in the test specimen at discrete points during the test. From these data, it is then possible to produce true-stress-true strain curves for the multiaxial stress states encountered in forming, rather than relying on curves derived from unixial tests.

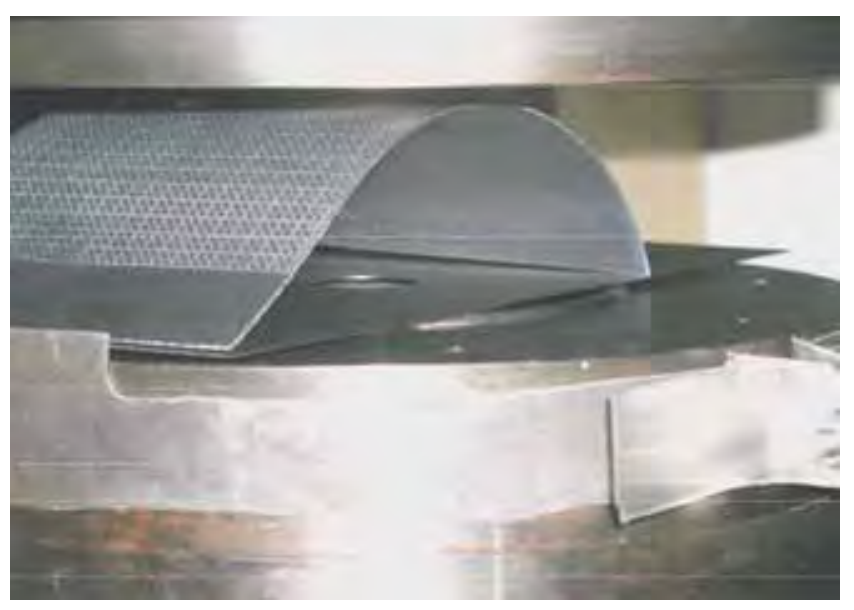

Figure 12: NUMISHEET benchmark sample in the Formability Station being loaded in plane strain.

Currently the Materials Performance Group members are producing materials property data for simulations of prototype parts in a benchmark forming process as part of the NUMISHEET 2005 Conference. 


\section{Kolsky Bar Facility}

The Materials Performance Group of the Metallurgy Division has developed a Kolsky or Split-Hopkinson bar facility (Figure 13) to measure the high strain-rate, high heating-rate constitutive response of metals to provide data for developing predictive models of these materials for use in finite element codes or other numerical analysis techniques. A key feature of the NIST Kolsky Bar is the ability to rapidly heat samples prior to testing. Sufficiently rapid heating can short-circuit normal diffusion-related microstructural changes that would ordinarily occur under quasi-equilibrium heating.

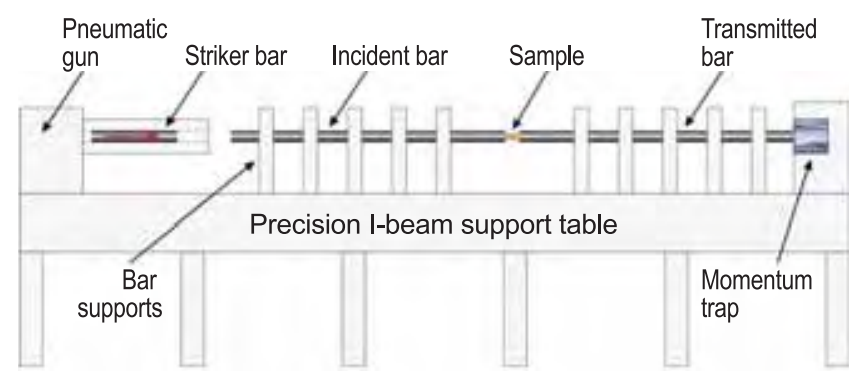

Figure 13: The Kolsky Bar Facility is equipped with a special pulse-heating capability (not shown) provided by conductive bearings at the bar supports nearest the sample.

As a result, the flow stress of rapidly heated materials can be much different than what is measured under quasi-equilibrium conditions. There is currently a lack of such high-temperature non-equilibrium constitutive data for most metals and alloys of interest. An important application of industrial interest is high-speed machining, where rapid temperature increases occur at the tool/workpiece interface.

\section{Magnetic Engineering Research Facility}

The Magnetic Materials Group of the Metallurgy Division has an elaborate facility specifically designed

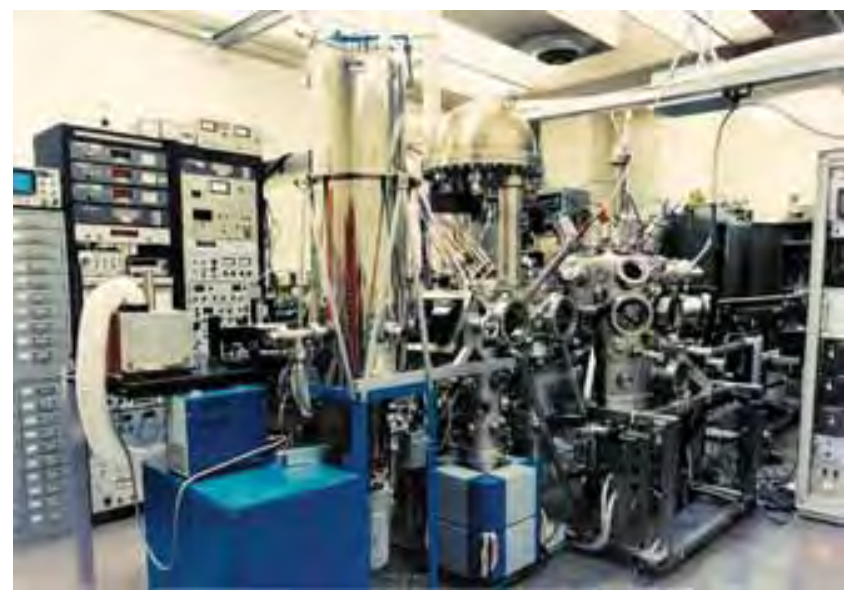

Figure 14: The Magnetic Engineering Research Facility is the world's most elaborately instrumented system for magnetic thin-film deposition and in situ characterization. for advancing key enabling technologies in the field of ultrahigh-density data storage. It has many unique features. Films can be deposited both by the method preferred in basic research (molecular beam epitaxy) and by the method of industrial manufacturing (magnetron sputtering). Numerous in situ characterization techniques are available including scanning tunneling microscopy, $\mathrm{x}$-ray photoelectron spectroscopy, Auger electron spectroscopy, ion scattering spectroscopy, low-energy electron diffraction, reflection high-energy electron diffraction, and mass spectrometry. For in situ magnetic measurements both a superconducting magnet and an electromagnet are available and are equipped for magnetoresistance and magneto-optical Kerr effect measurements.

\section{In Situ Stress Measurement Facility}

The Thin Film and Nanostructure Processing group of the Metallurgy Division has established a Class II $(1 \mathrm{~mW}) \mathrm{HeNe}$ optical bench dedicated to the in situ measurement of surface and growth stress during electrochemical processing using the wafer curvature

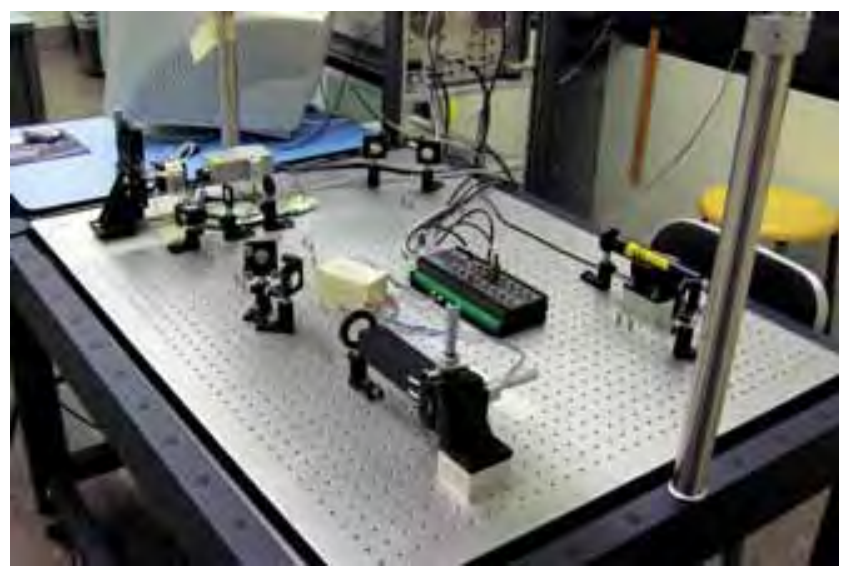

Figure 15: Optical bench showing components of in situ stress measurement system.

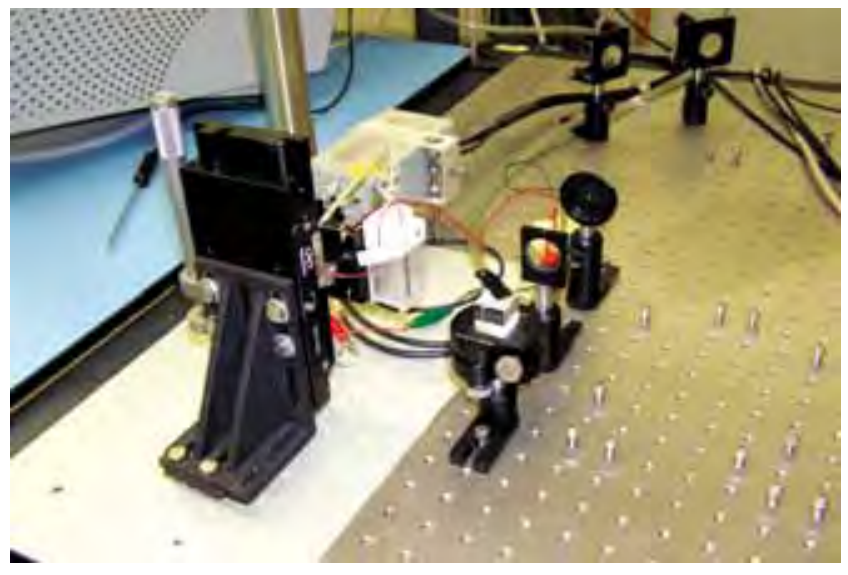

Figure 16: Close-up of electrochemical cell showing cantilever electrode. 
method. Surface stresses on the order of $0.008 \mathrm{~N} / \mathrm{m}$ (23 km radius of curvature for $0.1 \mathrm{~mm}$ thick borosilicate glass substrates) can be resolved while the beam is in solution, sufficient to study the adsorption of molecular monolayers onto the electrode surface. The curvature of the substrate is monitored during electrodeposition by reflecting the laser off of the glass/metal interface, through a series of mirrors, and onto a position-sensitive detector, Figures 15 and 16. Surface and internal stresses for metal films electrodeposited onto the substrate can be calculated from the deflection of the beam as a function of time.

Supporting an increasing number of users, there are several notable developments at the NCNR in: Sample Preparation and Characterization Laboratories, Commissioning of a Double Focusing Triple-Axis Spectrometer, Beginning installation of the High Intensity Multi-Axis Cold Neutron Spectrometer (MACS), Data Analysis, Visualization, and Modeling Software - DAVE II, and Deployment of Electronics for Large Detector Arrays.

\section{Sample Preparation and Characterization Laboratories}

The NCNR completed in 2005 the renovation of three, $7.3 \mathrm{~m}$ x $4.9 \mathrm{~m}$ (24 ft x $16 \mathrm{ft}$ ), laboratories, nearly doubling the space and equipment available for the preparation and characterization of samples for neutron scattering experiments by users and staff. Users preparing to come to the NCNR for an experiment can find detailed descriptions of available laboratory equipment and supplies on the NCNR's website at http://www.ncnr.nist.gov/userlab/.

One of the new laboratories is the result of a bioengineering research partnership between the NCNR and five universities, with partial funding from the National Institutes of Health. This Cold Neutrons for Biology and Biotechnology (CNBT) laboratory is equipped with a high-volume laminar flow hood designed for working with acids (e.g., for cleaning substrates); a $1.44 \mathrm{~m}^{3}\left(51 \mathrm{ft}^{3}\right)$ chromatography refrigerator; a large $-40{ }^{\circ} \mathrm{C}$ freezer; a temperature controlled, $13200 \mathrm{rpm}$ microtube centrifuge; as well as standard wet chemistry equipment for preparing biology-related samples for diffraction, reflectometry, and SANS experiments.

One laboratory is dedicated primarily to sample characterization and includes equipment for dynamic light scattering, infrared spectroscopy, UV-Visible Spectrophotometry, gas chromatography, and an optical microscope with temperature-controlled sample stage, camera, and attachments for fluorescence measurements.

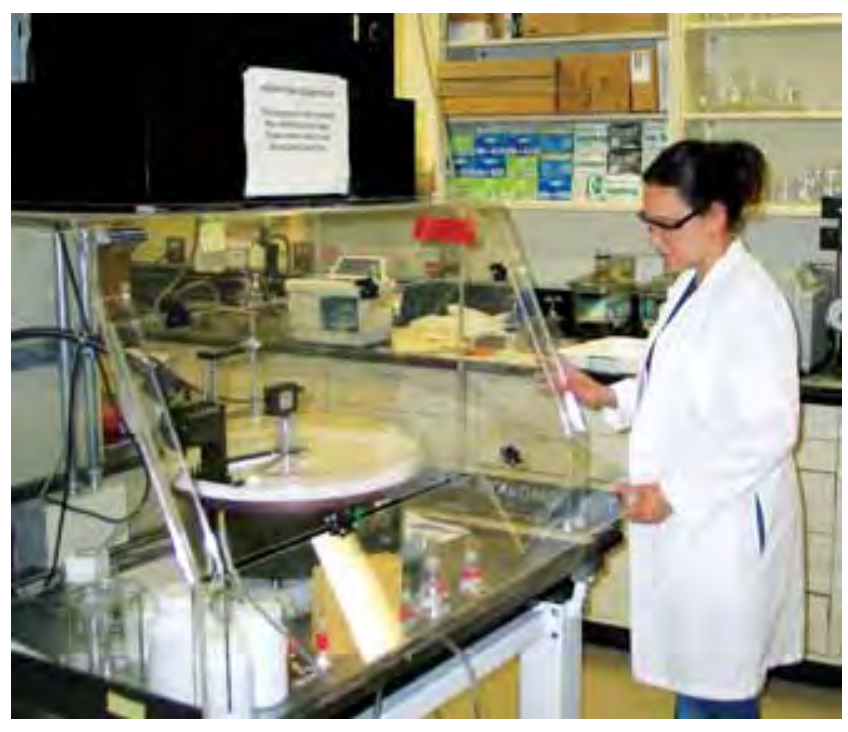

Figure 17: Yamali Hernandez keeps a watchful eye on the Langmuir-Blodgett trough in one of the NCNR's recently renovated user laboratories.

This laboratory also has a rotary evaporator for purifying solvents and a computer controlled Langmuir-Blodgett (LB) trough (see Figure 17) for depositing multi-molecular layer LB films on solid substrates.

In addition to the above, there are laboratories with specialized equipment for x-ray diffraction, solid-state chemistry, surface and interface science, and general solution chemistry. Also implemented this year is a bar-coding system for maintaining an accurate chemical inventory for all the laboratories. Users can now quickly obtain Materials Safety Data Sheets and determine the availability, location, and quantity of chemicals kept in any laboratory.

\section{Commissioning of a Double Focusing Triple-Axis Spectrometer}

The first measurements to characterize the performance of the new state-of-the-art thermal neutron triple-axis spectrometer at beam port BT-7 have been carried out and have met expectations. The new instrument features the choice of either a $\mathrm{Cu}(220)$ or PG(002) doubly-focusing monochromator, providing a continuous incident neutron energy range from $5 \mathrm{meV}$ to $500 \mathrm{meV}$. The $400 \mathrm{~cm}^{2}$ reflecting area for each monochromator yields as much as an order-ofmagnitude gain of neutrons onto the sample compared with other thermal triple-axis spectrometers at the NCNR. The reactor beam and post monochromatic beam elements (collimators, slits, and filters) offer a wide range of choices to optimize the resolution and intensity of the instrument, with available fluxes well into the $10^{8} \mathrm{n} / \mathrm{cm}^{2} / \mathrm{s}$ range.

The sample stage of the instrument includes two coaxial rotary tables, one for sample rotation and one 
for the independent rotation of magnetic field coils, and a computer-controlled sample goniometer and elevator. Polarized 3He cells are under construction for the instrument to provide full polarization analysis with either one of the monochromators and any of the standard analyzer crystals.

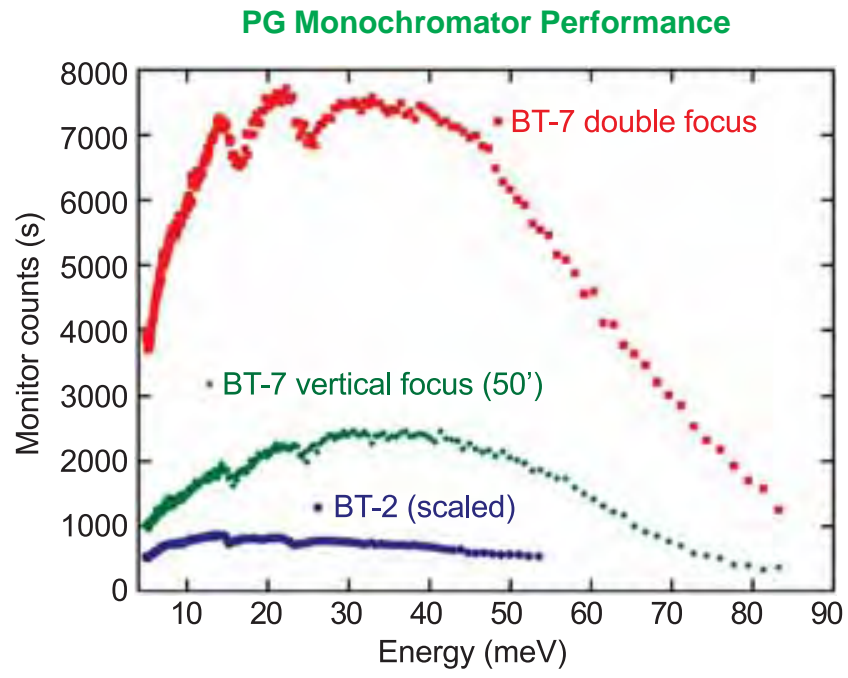

Figure 18: Comparison of the beam intensities (monitor count rates) for the PG(002) monochromators for the new BT-7 triple-axis spectrometer and the conventional triple-axis spectrometer at BT-2. The BT-7 monochromator features vertical focusing that varies with energy, whereas the BT-2 monochromator has a fixed vertical focus optimized for $14.7 \mathrm{meV}$. The collimation was open-50'before and after the monochromator, respectively, on both instruments. The much larger BT-7 monochromator provides more neutrons that fully illuminate the sample over a wider range of incident energies. The new double focusing mode is seen to provide a dramatic gain in neutron intensity by relaxing the wave vector resolution of the instrument.

The BT-7 spectrometer is designed to be used with interchangeable customized analyzer/detection systems supported on air pads. The first new analyzer system will have a multi-strip PG(002) analyzer array that can be used in a horizontally focused mode, or in a flat configuration either with a linear position-sensitive detector or with conventional Söller collimators. All options will be under computer control and can be selected and interchanged by the experimenter without requiring any mechanical changes or user intervention. A separate diffraction detector is provided in front of the analyzer for Bragg peak measurements, and a series of 13 detectors embedded in the shielding behind the analyzer will continuously monitor the neutron flux entering the analyzer system. These detectors can also be used for measurements of the instantaneous correlation function, for example, or with a radial collimator to determine a diffraction pattern over a limited angular range. This analyzer system is at an advanced stage of development and is scheduled to be installed early in 2006. In the interim, a conventional analyzer system has been installed on air pads so that the instrument is now fully operational as a triple-axis spectrometer, although the full measurement capability and flexibility won't be realized until the new analyzer system is available.

\section{Installation of the High Intensity Multi-Axis Cold Neutron Spectrometer (MACS) Begins}

While inelastic neutron scattering in principle can elucidate atomic-scale dynamics in almost any material, experiments on the most exciting new materials are often impractical because high-quality samples are too small. Arguably, this is not a fundamental limitation of the technique but due to the lack of flux and detection efficiency of current instrumentation. The MACS project aims to increase both factors by an order of magnitude to enable informative experiments on $1 \mathrm{mg}$ samples and comprehensive maps of inelastic scattering when larger samples are available.

MACS is a third generation cold neutron spectrometer and, as such, presents many fascinating engineering challenges. In the past year, most of these have been overcome, and MACS is now in the midst of a busy manufacturing and installation phase. Led by Donald Pierce, George Baltic, and Nick Maliszewskyj, the NCNR facilities engineering group has designed, procured, painted, and filled more than 50 metric tons (50 tons) of beamline shielding and beam conditioning optics for MACS.

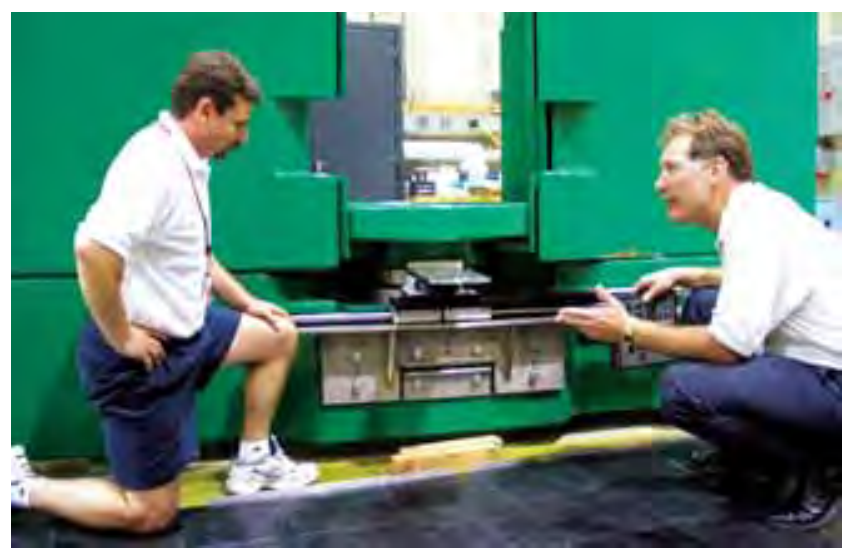

Figure 19: Peter Hundertmark and Timothy Pike examine the ball screw mechanism that will rotate the sample and detector around the green monochromatic beam transport system, which they designed.

Figure 19 shows parts of the monochromatic beam transport system for MACS along with Timothy Pike and Peter Hundertmark who designed it. The shielding penetration consists of an adjustable channel lined by $\mathrm{m}=3.5$ super mirrors that will guide a converging monochromatic beam to the sample position while minimizing the aperture for fast neutrons and gamma 
radiation. The ball screw actuation mechanism visible in Figure 19 will position the super mirror channel, the sample table, and the detector system to receive a monochromatic beam with energies varying from $2.5 \mathrm{meV}$ to $20 \mathrm{meV}$. Anticipating high-field superconducting magnet systems, all materials within $750 \mathrm{~mm}$ of the sample position are non-magnetic.

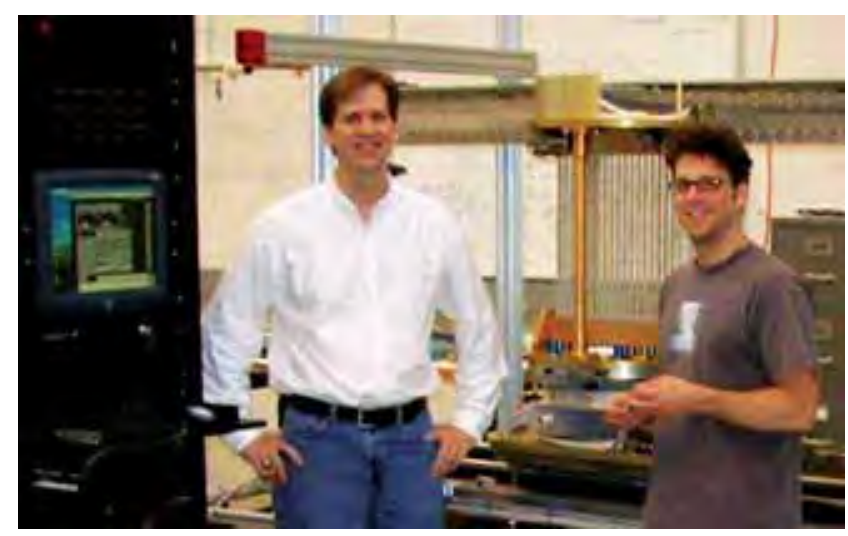

Figure 20: Stephen Smee (left) and Gregg Scharffstein with the MACS monochromating system. (The monochromator is between and behind them on the bench.) Not shown are Joe Orndorff and Randy Hammond who complete the team from the Instrument Development Group at Johns Hopkins University that designed and assembled the system.

Apart from the intense NCNR cold neutron source, the key to enhancing flux on sample is the monochromating system, which will focus the beam from $1400 \mathrm{~cm}^{2}$ to $8 \mathrm{~cm}^{2}$. It consists of a variable beam aperture to minimize background and to control wave vector resolution, a four-position radial collimation system to control energy resolution, and a translating, doubly focusing graphite monochromator. These will inhabit a helium-filled cask to reduce air scattering and pack shielding close to the complex beamline elements. Figure 20 shows these items during tests by the Instrument Development Group at Johns Hopkins University where they were designed and assembled. The monochromating system now stands ready for neutrons at the NCNR.

All the equipment described above was installed in the beamline in the late summer and early fall of 2005. Sample stage and detector system installation will start in the fall so MACS can be ready for commissioning experiments and friendly users in the spring of 2006. While MACS will eventually become a Center for High Resolution Neutron Scattering (CHRNS) user facility for a broad range of science spanning condensed matter physics, chemistry, and biology, principal investigators Collin Broholm and Jeff Lynn are particularly excited about the new capabilities that MACS will provide for research in quantum magnetism and superconductivity.

\section{Data Analysis, Visualization, and Modeling Software - DAVE II}

Significant development activity in the Data Analysis and Visualization Environment (DAVE) team over the last year has resulted in a brand new look and feel for DAVE. DAVE II brings a new paradigm to data visualization and analysis via a novel data-centric user-interface. The infrastructure includes a project manager, data manager, visualization manager, user-interface, visualization modules, and mechanisms for easy integration of additional applications as they become available. All of this has been designed to help the user get the most from his/her neutron data.
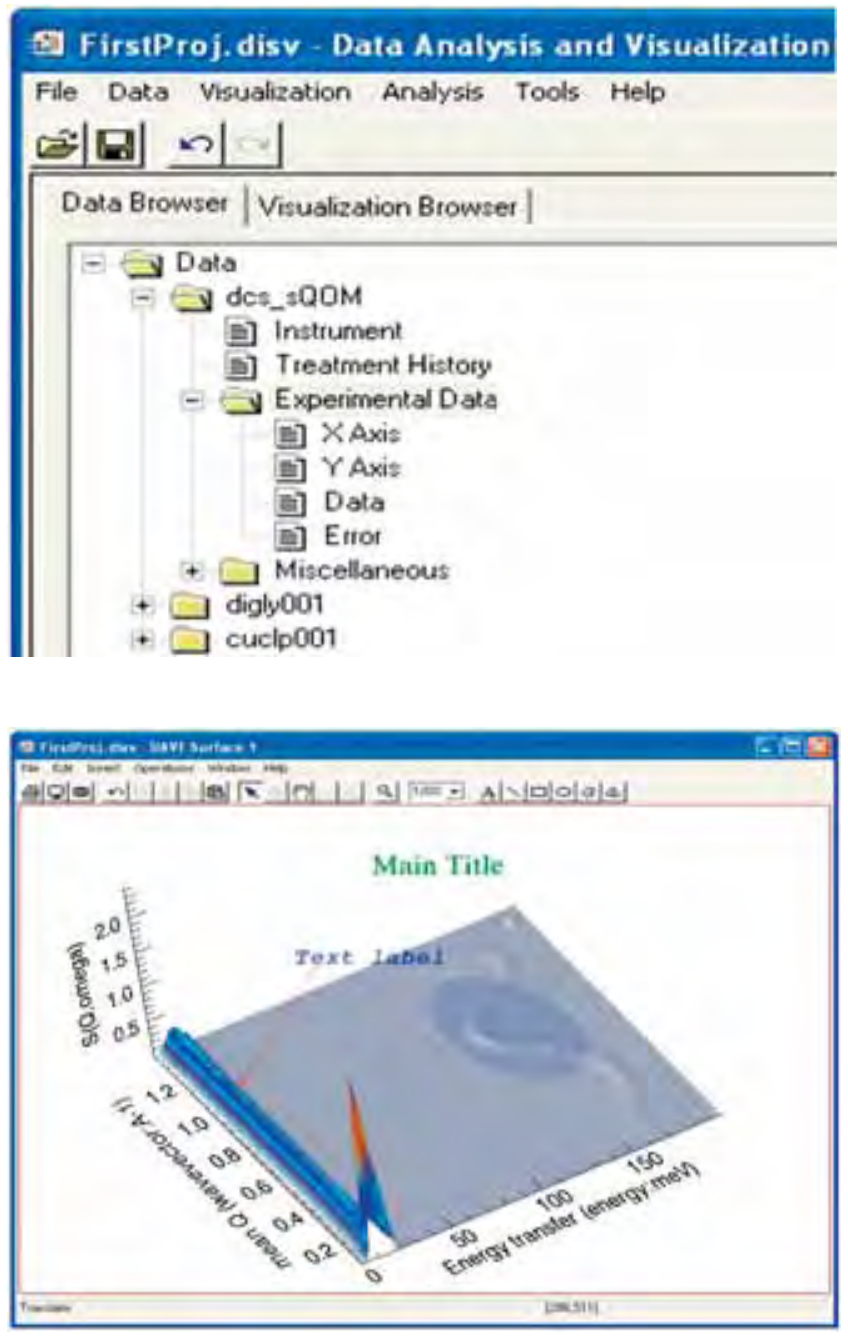

Figure 21: Data in DAVE II can be imported, manipulated, and displayed in easily customizable yet sophisticated plots.

The project manager concept promises to greatly simplify the user experience because it allows a user to save a complete session (plots, reduction steps, data operations, etc.) and restore it at a later time. Moreover there is much greater flexibility in DAVE II with the implementation of a document/view architecture. 
Multiple datasets can be loaded simultaneously and multiple views (even of the same dataset) can be created (line, contour, image, surface and volume renderings using OpenGL, the industry standard for high performance graphics). The visualizations are feature-rich allowing simple creation of sophisticated plots resulting in publication-quality output. Undo/redo functionality is implemented allowing scientists to perform what-if types of analyses with ease, thus enhancing the user experience. In the initial release, support for ASCII and the current DAVE format are provided. Operations to perform specific data analysis tasks are being implemented, and the initial data reduction functionality is identical to that of the current version of DAVE.

\section{Deployment of Electronics for Large Detector Arrays}

As the number of detectors on an instrument increases and the detectors themselves are buried in locations that are not readily accessible, the task of tuning the discriminator thresholds and periodically inspecting the banks for quality control becomes a major undertaking. The Preamplifier-AmplifierDiscriminator 2 (PAD2) system (Figure 22), developed by the NCNR's Jeff Ziegler and Nick Maliszewskyj, is a state-of-the art solution to the needs of instruments with a large number of detectors. This system consists of compact front-end modules combining the functions of preamplifier, amplifier, and discriminator; concentrator boards that multiplex control and data signals for eight front-end units; and a system

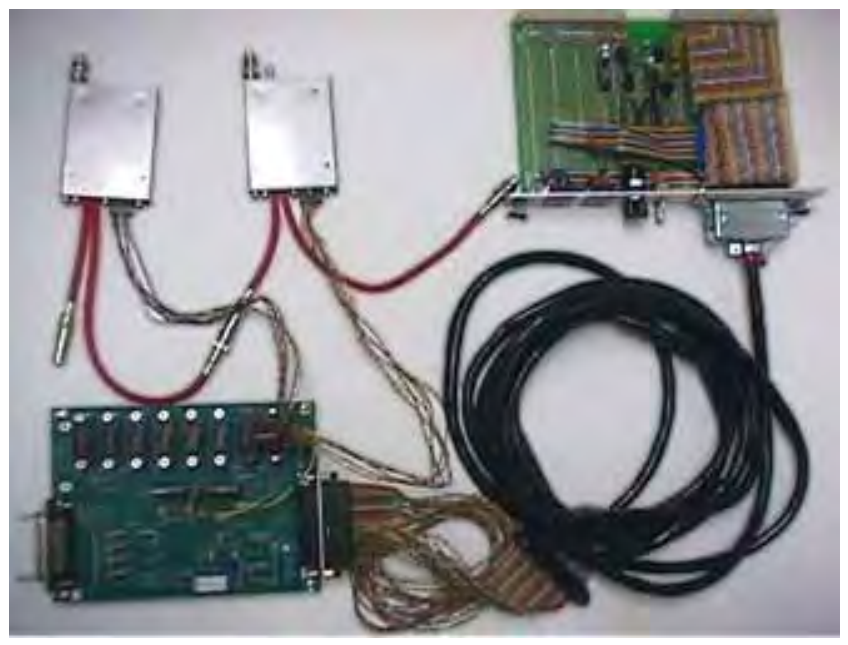

Figure 22: Preamplifier-Amplifier-Discriminator 2 (PAD2) system components.

controller that can set the gain and signal threshholds for a collection of up to 2048 individual detectors. The differential signal levels employed allow for longer cable runs and greatly improved noise rejection over the existing TTL solutions. Live quality control is now possible thanks to in situ pulse height analysis. A single point of control makes it possible to tune the discriminator settings for detectors behind shielding or in vacuo. Taken in combination, the capabilities of this new system are groundbreaking and surpass anything available commercially. The system was successfully deployed on the 32-detector BT-1 powder diffractometer and the HFBS backscattering spectrometer in 2005. 


\section{Databases}

\section{SRD 31: Phase Equilibria Data T.A. Vanderah}

The Phase Equilibria Data project is a joint effort of NIST and the American Ceramic Society (ACerS). Working together, NIST and ACerS have published more than 20,000 diagrams depicting graphical representations of the regions of distinct chemical and structural behavior of materials in thermodynamic equilibrium. The diagrams are distributed by ACerS in traditional printed volumes and in a modern searchable PC database system. Phase Equilibria Diagrams, Volume XIV, Oxides, completed this year and unveiled at the annual ACerS meeting in April, contains figures numbered from 10841 to 11637 and covers oxide systems originally published in the literature between 1980 and 2004 and not previously included in the series.

\section{SRD 83: NIST Structural Database V.L. Karen}

The NIST Structural Database (NSD) contains evaluated crystallographic data (lattice parameters, atomic positions, and symmetry classifications) for metallic crystalline substances, including alloys, intermetallics, and minerals. The data set is licensed for use in both personal computers and instrumentation. This year's updates doubled the accessible data to more than 16,000 records.

\section{SRD 84: FIZ-NIST Inorganic Crystal Structure Database V.L. Karen}

The Inorganic Crystal Structure Database (ICSD) is produced cooperatively by the Fachinformationszentrum Karlsruhe (FIZ) and the National Institute of Standards and Technology (NIST). The ICSD is a comprehensive collection of crystal structure data for inorganic compounds. In addition to the full three-dimensional crystal structure data, chemical composition, reduced cell symmetry data, and bibliographic information, the ICSD includes enhanced features for the characterization of materials based on lattice and chemistry search modules, 3-dimensional visualization, and powder pattern simulation of inorganic structures. Two updates this year added approximately 2000 entries and brought the total number of entries to more than 70,000.

\section{SRMS}

The Ceramics Division is the world's leading producer of x-ray Certified Reference Materials. Last year, determination of the amorphous content of NIST Standard Reference Material ${ }^{\circledR}$ (SRM) 676 (the primary internal standard for quantitative analysis by x-ray diffraction) launched a recertification effort for a suite of additional SRMs also utilized in quantitative analysis. Recertification now has been completed for SRMs 656 (two mixtures of $\alpha$ and $\beta$ phases of silicon nitride), 674b (oxide powders for internal intensity standards), and 1878a (respirable quartz) and 1879a (respirable cristobalite) for quantitative analysis in occupational safety and health applications. 


\section{Recommended Practice Guides}

\section{SP960-1: Particle Size Characterization Ajit Jillavenkatesa, Stanley J. Dapkunas, and Lin-Sien H. Lum}

The consequences of improper particle size analyses include poor product quality, high rejection rates, and economic losses. Hence, the ceramic manufacturing industry and the broad range of applications utilizing powders have a strong and active interest in the metrology required for accurately determining the distribution of particle sizes in a powder. This Guide describes the techniques most commonly used for measurement of particle size and size distribution, including sieving, gravitational sedimentation, laser light diffraction, and microscopy-based methods. The capabilities and limitations of these techniques are examined together with discussions of the general principles on which the techniques are based. The Guide includes discussions of the fundamental issues in representative sampling, the procedures and precautions generally followed for sample preparation and analysis, and the assessment of sources of error, bias, and other potential variations in the measurement results.

\section{SP960-2: The Fundamentals of Neutron Powder Diffraction John R.D. Copley}

This Recommended Practice Guide introduces the reader to neutron powder diffraction, a powerful technique that complements $\mathrm{x}$-ray diffraction as a means to determine crystal structures. The principal differences between the x-ray and neutron techniques are discussed, and several examples of applications to materials of industrial interest are described.

\section{SP960-3: The Use of Nomenclature in Dispersion Science and Technology Vincent A. Hackley and Chiara F. Ferraris}

Measurements and standards enhance reliability in manufacturing by providing a common basis for quantifying and comparing material properties during each phase of the manufacturing process, from raw materials to the finished product. Underlying this ability, and essential to its success, is the use of a well-defined, widely accepted, and uniformly applied nomenclature. An established nomenclature significantly enhances the accuracy and efficiency of the descriptions of experimental methods and instrumentation, facilitates the sharing of technical ideas and concepts, and provides a sound basis on which to standardize measurement methods and data reporting practices. This Guide was prepared as a resource for researchers, engineers, and students working on dispersion-based applications. While emphasizing commonly encountered terms, every effort was made to maintain a degree of uniformity with existing standards and conventions to provide a consistent framework for improved technical communication. The result was a comprehensive glossary of terms related to the central issues in dispersion science and technology.

\section{SP960-4: Installing, Maintaining, and Verifying Your Charpy Impact Machine Daniel P. Vigliotti, Thomas A. Siewert, and Chris N. McCowan}

The quality of the data developed by pendulum impact machines depends on how well the machines are installed, maintained, and verified. This is the reason that ASTM Standard E 23 Standard Test Methods for Notched Bar Impact Testing of Metallic Materials specifies annual direct and indirect verification tests. Each year, NIST provides reference specimens for indirect verification of over 1000 machines around the world. From evaluation of the absorbed energies and the fractured specimens, we attempt to deduce the origin of energies that are outside the ranges permitted by Standard E 23 and report these observations back to the machine owners. This Recommended Practice Guide summarizes the bases for these observations and, hopefully, will allow machines to be maintained at higher levels of accuracy. In addition, we provide details of the NIST verification program procedures and the production of the specimens.

\section{SP960-5: Rockwell Hardness Measurement of Metallic Materials Samuel R. Low}

The Rockwell hardness test continues to be applied as a tool for assessing the properties of a product while the tolerances on the acceptable material hardness have become tighter and tighter. Adhering to "good practice" procedures when performing Rockwell hardness measurements and calibrations is a beneficial step to reducing measurement errors. The purpose of this Guide is to explain the causes of variability in Rockwell hardness test results and to supplement the information given in test method standards with good practice recommendations. Although this Guide is directed more towards the users of Rockwell hardness having the greatest concern for accuracy in their measurements, much of the information given is also applicable for users that only require test results to be within wide tolerance bands, where high accuracy is not as critical. 


\section{SP 960-7: Capacitance Cell Measurement of the Out-of-Plane Expansion of Thin Films Chad Snyder and Frederick Mopsik}

This Guide describes the construction, use, and data analysis methods for a capacitance-based metrology for robust and highly accurate measurement of the out-of-plane expansion of films between $2 \mu \mathrm{m}$ and $1 \mathrm{~cm}$ in thickness. This metrology was developed because the coefficient of thermal expansion (CTE) continues to be a critical design parameter for the semiconductor electronics industry and is increasingly important to nanotechnology applications, as it is needed to estimate the stresses generated by thermal differentials. In response to a lack of robust methods for measuring the out-of-plane expansion of thin polymer films in the thickness range between the capabilities of typical thermomechanical analyzers (TMA) and x-ray reflectometers, NIST developed this capacitance-based measurement technique. This guide closely examines the sources of error associated with the metrology, and the descriptions are sufficiently detailed that the reader should be able to reproduce the metrology and achieve its typical measurement reproducibility upon thermal cycling of $\pm 2 \mu \mathrm{m} / \mathrm{m}$.

\section{SP960-8: Test Procedures for Developing Solder Data}

\section{Thomas A. Siewert and Carol A. Handwerker}

This publication documents standardized test procedures that can produce valid and reproducible mechanical-property data for lead-free solders. Such data speeds the application of lead-free solders in high-volume, automated production of electronic assemblies, especially when current production expectations combine high levels of quality with the lowest cost. Use of standardized procedures facilitates the comparison of data between laboratories and permits the combination of data from different sources into a single, comprehensive database.

Most dimensions and temperatures are listed without tolerances. Unless otherwise specified, use $\pm 5 \%$ on dimensions, times, and pressures, and $\pm 3{ }^{\circ} \mathrm{C}$ for temperatures.

Many of the procedures assume some skill in the arts of specimen production and testing. Various textbooks and industry brochures (some listed in the Bibliography) can provide background information on these skills and any hazards associated with these procedures.

\section{SP960-9: Surface Engineering Measurement Standards for Inorganic Materials Stanley J. Dapkunas}

Applications as diverse as medical implants and gas turbines have been the beneficiaries of the substantial accomplishments in the surface engineering of materials. Characterizations of surfaces and measurements of surface properties have become vital to systems design, maintenance, and analysis and, hence, to the commerce that depends on those systems. This Guide identifies pertinent properties and characteristics of engineered surfaces and provides descriptions of the most prevalent standard test methods applied to the measurement or assessment of those characteristics. The type of data produced using each method is described as well as the specimen requirements, the intended application, and the limits of the method. An extensive cross index of key words allows identification of standards by method, property, or type of material.

\section{SP960-10: X-Ray Topography David R. Black and Gabrielle G. Long}

The study of the interrelationships among processing, structure, and properties is fundamental to the field of materials science and engineering. The important role of microstructure in these relations has driven the development of a wide variety of x-ray based characterization techniques, including the technique of x-ray topography (XRT). This powerful tool provides a nondestructive means of imaging the defect microstructure of crystals for defects in the size range of micrometers to centimeters. This technique, however, has been underutilized in the United States. Consequently, this guide was developed to make the technique more accessible to materials scientists who can benefit from the rich variety of microstructural information it offers. The basic principles and practical aspects of topography are discussed, and numerous examples are presented to illustrate the diversity of applications that can benefit from this technique.

\section{SP960-11: Data Evaluation Theory and Practice for Materials Properties Ronald G. Munro}

Technology thrives on data, and advances in science are propelled by advances in data. It follows that the quality of the available data is a concern of central importance to all of science and engineering. The process by which collections of data are assessed with respect to reliability, completeness, and consistency is 
known as data evaluation. This guide addresses data evaluation for materials properties as a scientific discipline that evolves from the formal underpinnings of materials metrology. After carefully establishing a theoretical foundation, an extensive collection of examples is used to examine, in succession, the issues of accessibility, reproducibility, consistency, and predictability. Distinctions are made among definitive relations, correlations, derived and semi-empirical relations, heuristic theories, and value estimates. Special subtopics include the use of properties as parameters in models, the interpretation of ad hoc parameters, and the treatments of procedural properties, response dependent properties, and system dependent data. The principles and practices set forth in this work serve as a guide to data evaluation across many disciplines, wherever considerations of numeric property data are crucial to the interpretation and application of quantitative observations.

\section{SP 960-13: Pore Characterization in Low-k Dielectric Films Using X-ray Reflectivity: X-ray Porosimetry Christopher L. Soles, Hae-Jeong Lee, Eric K. Lin, and Wen-li Wu}

This recommended practice guide describes the equipment and methodology for quantifying the average pore size, pore size distribution, and wall density in thin films where the sample mass is prohibitively small for traditional porosimetry measurements. This method was initially developed for nanoporous low-dielectricconstant thin films needed for the further advancement of semiconductor devices where in-house structural characterization methods are needed to evaluate candidate materials and processing methods, but can be easily generalized to other nanoporous thin films. The developed porosimetry method uses X-ray reflectivity to measure the density of the film while gradually increasing or decreasing of the partial pressure of condensate vapors such as toluene in the presence of the film. The change in film density can be directly related to the amount of adsorbed condensate, and thus porosity, if the density of the condensed fluid is known. Monitoring the amount of adsorbed condensate as a function of the partial pressure defines a physisorption isotherm, the basic starting point for any number of analytical interpretations. The X-ray Porosimetry Recommended Practice Guide provides detailed information so that this knowledge and expertise may be transferred to industrial research and university laboratories. It is hoped that X-ray porosimetry will be widely adopted for the evaluation and characterization of newly developed nanoporous thin-film materials. 




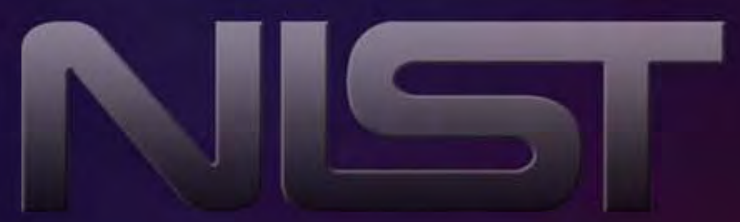

